\title{
Abstracts From the 16th International Congress on Twin Studies and the 4th World Congress on Twin Pregnancy, Madrid, Spain
} November 16-18, 2017

\author{
HERITABILITY OF BIRTH SIZE: A POOLED \\ INDIVIDUAL-BASED ANALYSIS OF GLOBAL GEOGRAPHICAL \\ DIFFERENCES USING CODATWINS PROJECT \\ Yoshie Yokoyama for the CODATwins Project \\ Osaka City University, Osaka, Japan
}

Introduction: Birth size is an indicator of infant health and also associated with a wide range of health related traits in later life such as higher blood pressure and obesity. Moreover, there is clear evidence that low birth weight, compared to normal birth weight, is associated with a higher risk of metabolic diseases including type 2 diabetes and cardiovascular diseases in adulthood. Genetic and environmental variation of fetal growth may differ between populations because of differences in maternal dietary habits, other environmental exposures and the gene pool of population. We examined differences in the genetic and environmental contributions to birth weight, length, and ponderal index (PI) across geographic-cultural regions (Europe, North America and Australia, and East Asia) and how gestational age modifies these effects. Methods: Data from 26 twin cohorts in 16 countries including 57,613 monozygotic and dizygotic twin pairs were pooled. Genetic and environmental variations of birth size were estimated using genetic structural equation modeling. Birth weight, length and PI values (both unstandardized and standardized for gestational age) were first adjusted for twin cohort within each sex and geographic-cultural region using linear regressions, and the resulting residuals were used in the analyses. All genetic models were fitted by the OpenMx package in the $\mathrm{R}$ statistical platform using the maximum likelihood method to estimate the variance components with $95 \%$ confidence intervals (CIs). Results: Additive genetic factors explained a small proportion of the total variance of birth size (up to 23\%), whereas the contribution of environmental factors, shared by co-twins, was substantially larger (up to $65 \%$ ). After standardization for gestational age, the proportions of shared environmental variances of birth weight decreased from $65 \%$ to $30 \%$, and for birth length and PI from $74 \%$ and $45 \%$ to $50 \%$ and $43 \%$, respectively. The heritability estimates were similar in the geographic-cultural regions, but estimates for unique environmental influences were slightly larger and shared environmental influences smaller in East Asia than in the other regions. Conclusions: The contribution of genetic factors to birth size is around 20 to $25 \%$, which is smaller than the estimate for the contribution of shared environmental factors. Shared environmental factors have an important role in birth size and these effects differed across geographic-cultural regions.
ASSOCIATION OF CERVICAL LENGTH CHANGE AFTER INITIAL INDUCTION OF LABOR WITH SUBSEQUENT SUCCESSFUL INDUCTION IN TWINS

Song Yi Kook, Kyo Hoon Park, Se Jeong Jeon, Hyun Soo Park, and Ha-Na Yoo Department of Obstetrics and Gynecology, Seoul National University College of Medicine, Seoul National University Bundang Hospital, Seongnam, Korea

Introduction: Over half of the women with twin gestations attempting vaginal delivery require induced labor. However, there is little information regarding the ultrasonographic parameters and clinical which predict the success of labor induction. Recent published studies involving twin pregnancies showed that the change in cervical length over time was associated with preterm delivery. Therefore, we considered that the possibility that similarly in women who failed to induce labor on the first attempt of serial induction, the change in cervical length following the attempt may be predictive of the induction success on the second attempt. The purpose of this study was to determine whether the change in cervical length is valuable in predicting the success of serial induction of labor on the second day in women with twin gestations who failed to induce labor on the first day. Methods: This retrospective cohort study included 72 consecutive women with twin gestations at $\geq 35.0$ weeks of gestation who failed labor induction on the first day of serial induction. Cervical length on transvaginal ultrasound and the Bishop score were estimated prior to performing each labor induction on the first and second days. The primary outcome measure was successful labor induction (defined as an ability to achieve the active phase of labor). Results: The overall success rate of labor induction performed on the second day was $36.1 \%$ (26/72). The group of women in whom labor was successfully induced on the second day had a significantly higher mean change in cervical length and maternal height compared with the group of the women in whom labor induction failed. However, other parameters studied, including cervical length and Bishop score on day 2, change in Bishop scores, chorionicity, and methods of conception, were not significantly different between the two groups. Multiple logistic regression analysis identified change in cervical length and maternal height as independent predictors 
of the successful induction on the second attempt. Conclusions: A greater change in cervical length is a good predictor of successful labor induction on the second day in women with twin gestations in whom induction failed on the first day.

\section{ORO-DENTAL COMPARO IN TWINS}

\section{S. Bharath Vardhana}

Department of Pediatric Dentistry, Subbiah Dental College, Hospital \& Research Center, Shimoga City, Karnataka State, India

Aim(s) of the Study: The study of twins provides a unique opportunity to evaluate the genetic and non-genetic contributions to a variation in the multitude of traits. Twin research has revealed the impact of a strong genetic component to the variance of many dental traits. The present study was conducted in monozygotic (MZ) and dizygotic (DZ) twins to assess the (all intra-pair comparisons): (1) salivary $\mathrm{pH},(2)$ oral hygiene status, (3) dental caries, (4) occlusion (type of contact of upper and lower teeth) and (5) dental arch measurements (width and length of the upper and lower arches). Methodology: A random sample of $129 \mathrm{MZ}$ and DZ twin pairs aged 6-15 years from Bangalore, India were included in this study. The zygosity of the twins was initially recorded by facial appearance and later determined by dermatoglyphics. The assessment of oral parameters such as salivary $\mathrm{pH}$, oral hygiene status, dental caries status, occlusion and dental arch measurements was performed in these twin pairs in the school with necessary permissions obtained. The data were recorded and subjected to statistical analysis. Results: (1) A moderate degree of agreement between zygosity determination by comparison of facial appearance and dermatoglyphics was found; (2) The mean salivary pH, OHI-S (Oral hygiene index) scores, deft and DMFT (caries), occlusal status and dental arch measurements were found to show no statistically significant difference in MZ twin pairs; (3) The DZ twin pairs did not show any statistically significant difference in the oral parameters studied except for the dental arch measurement (especially the mandibular arch length), which showed a moderate degree of significance. Interpretation and Conclusion: It was concluded from the present study that there were similarities in the dental traits observed in the monozygotic (intrapair comparison) and dizygotic twin pairs (intra-pair comparison) with a chance variation in one of the parameters in DZ twin pairs indicating a genetic influence on these traits. The determination of zygosity by dermatoglyphics seems to be a non-invasive and an acceptable method of determination of zygosity as evidenced by this study. (Although blood sampling would have been ideally indicated for zygosity determination. Due to local administrative difficulties this methodology could not be performed.)

\section{A GENOME-WIDE PROFILING OF EPIGENETIC FINGERPRINT IN ADULT TWINS OF PRETERM BIRTH}

Qihua Tan ${ }^{1,2}$, Shuxia $\mathrm{Li}^{2}$, Morten Frost ${ }^{3}$, Marianne Nygaard', Mette Soerensen', Kaare Christensen ${ }^{1,2}$, and Lene Christiansen'

${ }^{I}$ Epidemiology and Biostatistics, Department of Public Health, University of Southern Denmark, Odense, Denmark

${ }^{2}$ Unit of Human Genetics, Department of Clinical Research, University of Southern Denmark, Odense, Denmark

${ }^{3}$ Department of Endocrinology, Odense University Hospital, Denmark

Preterm birth is a leading cause of perinatal mortality and longterm health consequences. Epigenetic mechanisms may have been involved in preterm birth which could be persistent and detrimental to health later in life. We performed a genome-wide DNA methylation profiling of premature birth to identify genomic regions under steady differential epigenetic regulation in 144 adult twins with a median age of 33 years (age range: 30-36). Association analysis detected four regions reaching genome-wide significance (FWER $<$ 0.05 ) that were annotated to SDHAP3, TAGLN3, PLCXD2, ZNRD1, EBF3 and TCERG1L genes on chromosomes 5, 3, 6, and 10. These genes display strong involvement in neurodevelopmental disorders and cancer susceptibility. The identified regions were replicated in two independent samples of even older age with the regions on chromosomes 5 and 3 double replicated, on chromosome 6 single replicated. Gene set enrichment analysis detected a family of the phase II detoxification genes reportedly contribute to adverse pregnancy outcomes. The findings from adult twins of premature birth provide molecular evidence of long-term effect of early life events on disease and health.

\section{MULTIPLE-BIRTH SINGLE PARENTS EXPERIENCES OF THEIR DAILY LIFE}

\section{Kristiina Heinonen}

Department of Nursing Science, University of Eastern Finland, Savo Vocational College, Kuopio, Finland

Introduction: The research is a part of the ICOMBO research. A single parent family is a family where most of the daily life depends on the one parent. This data describes participants' experiences from Australia, United Kingdom, United States, New Zealand, Canada, South-Africa and Ecuador. Materials and Methods: The aim was to describe what it is like to be a single parent in a family with more than one child of the same age. The data was gathered for this research using a specially created questionnaire with structured and open questions. In a accordance with research ethics, the participants received information on the research before sending their answers through the websites of multiple-birth organizations and by answering the questionnaire they gave their informed consent to participate in this study. The open questions analysis based mostly on hermeneutic phenomenology. The concept of the lifeworld has been used to describe the experiences of the participants. Results: The lifeworld of being a single parent in multiple-birth family can be described as 'Moving forward' as a temporal view, 'Being surrounded by demands' as a bodily view, 'Diversity of relationships' as a relational view and 'A different experience of the world' as a spatial view. All these are intertwined and interact with each other. Conclusion: The daily life of a single parent in multiple-birth families is individual and involves special needs. The parent is entitled to have received targeted support from professionals. The lifeworld before and after the divorce may be fragmented and a single parent will need to put the pieces back together again. Despite this, the new situation can bring a feeling of security and more confidence in the future. The decision to become a single parent is closely related to concern about the safety and future of the children. The family is also a part of society. Different kind of social and health care professionals should give more attention to multiple-birth single-parent families. There is a need for education and better training for professionals. Support networks and digital support should be improved because the life situations are different. A useful topic of further research would be the experiences of family members and professionals.

\section{THE EVERYDAY EXPERIENCES OF FINNISH}

\section{MULTIPLE-BIRTH SINGLE PARENTS}

Kristiina Heinonen

Department of Nursing Science, University of Eastern Finland, Savo Vocational College, Kuopio, Finland

Introduction: This study is part of the ICOMBO research. A singleparent family is one in which the children are looked after by one of their parents, who do not live together. This data describes the experiences of participants in Finland. Materials and Methods: The aim was to describe what it is like to be a single parent in a family with more than one child of the same age. The data was gathered for this research using a specially created questionnaire with structured and open questions. In accordance with research ethics, the participants received information on the research before sending their answers 
through the websites of multiple-birth organizations, and by answering the questionnaire they gave their informed consent to participate in this study. The analysis of the open questions was based mostly on hermeneutic phenomenology. The concept of the lifeworld was used to describe the experiences of the participants. Results: The lifeworld of being a single parent in multiple-birth family can be described as 'Moving forward' as a temporal view, 'Being around required things' as a bodily view, 'Diversity of relationships' as a relational view and 'Surrounded by a supportive society' as a spatial view. All these are intertwined and interact with each other. Conclusion: The daily life of a single parent in a multiple-birth family is individual and involves special needs, but a supportive society can help in this situation. The parent is entitled to receive targeted support from professionals. The decision to become a single parent is closely related to concern about respecting childhood and sharing parenthood so that the children still have two responsible parents in their lives. It also involves feeling alone, missing companionship and fears, which also bring uncertainty into life. Despite this, the new situation is accompanied by the feeling that this was the right decision for everyone. Social and health care professionals should give more attention to such families. There is need for education and better training for professionals. Support networks and digital support should be improved because the life situations are different. The experiences of family members and professionals would be useful topics for further research.

\section{BIRTH WEIGHT DISCORDANCE AND ADVERSE PERINATAL OUTCOMES}

Shayesteh Jahanfar' and Kenneth Lim²

${ }^{I}$ Central Michigan University, USA

${ }^{2}$ Department of Feto-Maternal Medicine, Royal College of Women, Canada

Objective: Our purpose was to examine the association between discordant growth and perinatal mortality and morbidity among twins with careful adjustment for maternal and placental confounding factors. Study design: Two cohorts of twins with and without chorionicity data were analyzed and compared using generalized estimating modeling. Results: Our population-based dataset without chorionicity consisted of 12656 individual twins from which 4\% (452/12656) had $\geq 30 \%$ growth discordance. A subgroup of this population with chorionicity information had an incidence of $7.3 \% \geq 30 \%$ growth discordance. In growth discordant $(>30 \%)$ twins, perinatal mortality and morbidity were higher than concordant pairs $(p<.01)$. Low Apgar score and perinatal mortality were more frequent in growth discordant twins compared with growth concordant (all $p<.01$ ). Adjusted for chorionicity, the odds of composite perinatal morbidity variable were $59 \%$ higher in growth discordant twins compared with the reference category. Conclusion: Birth weight discordance places twins at increased risk for perinatal mortality. Growth discordance was also an independent risk factor for NICU admission and utilization of its resources for respiratory support.

\section{PRENATAL OUTCOMES AND BIRTH WEIGHT} DISCORDANCE, A SYSTEMATIC REVIEW

Shayesteh Jahanfar

Central Michigan University, USA

Both incidence and association of birth weight discordance (BWD) with adverse fetal and neonatal outcomes were reviewed. Searches were conducted using eleven databases inclusive of Medline, Cochrane Central Register of Controlled Trials, EMBASE, CINAHL, Database of Abstracts of Reviews of Effects, Web of Science, EBSCO, Paper First, Proceeding First, Web of Science, and Pro Quest Digital. More than 2,500 studies were screened, describing BWD specific to twin gestation, as a risk factor associated with fetal and neonatal outcomes. Forty-one articles, containing data from approximately 1.2 million twin pairs, were selected and assessed for quality. Twelve meta-analyses were performed and their heterogeneities (I2) were tested. A fixed model was generated if I2 $<25 \%$, while a random-effect model was adopted if $\mathrm{I} 2>50 \%$. Results: These were computed as relative risk and $95 \%$ confidence interval. BWD was found to be common among twin gestations, ranging from $8.0 \%$ to $58.2 \%$. It was found to be associated with nine outcomes, including stillbirth, early and late neonatal mortalities, length-of-stay greater than two days in neonatal intensive care unit, congenital anomalies, fifth minute Apgar score $<7$, newborn septicaemia, hypoglycaemia, and respiratory distress syndrome. The association between BWD and neonatal anemia was found to be insignificant. A wide range of incidence rate was found for the BW. It was concluded that exposure to growth discordance in twin gestation is significantly associated with perinatal mortality and morbidity.

\section{MENTAL HEALTH FOR MULTIPLE BIRTH FAMILIES: AN ANTENATAL GROUP FOR PARENTS EXPECTING MULTIPLES AND A POSTNATAL GROUP FOR PARENTS OF MULTIPLES}

G. Little', K. Bingham ${ }^{2}$, P. Anderson ${ }^{3}$, K. Hamilton ${ }^{3}$, M. Stuckey ${ }^{4}$, A. Clifford ${ }^{4}$, and C. Zanetti ${ }^{4}$

${ }^{1}$ Helios Psychology, Perth, WA, Australia

${ }^{2}$ Perth \& Districts Multiple Birth Association, Perth, WA, Australia

${ }^{3}$ St John of God Raphael Centre, Perth, WA, Australia

${ }^{4}$ The Elizabeth Clinic, Perth, WA, Australia

Mental health risk is greater for multiple birth parents than for parents of singletons in the perinatal period. This includes depression, anxiety and stress for mothers, anxiety and stress for fathers. Multiple birth families have also been found to have a greater risk of marital breakdown. The possible resultant impact on the developing babies is paramount. Perth and Districts Multiple Birth Association partnered with SJOG Subiaco, Raphael Services to deliver both an antenatal and a separate postnatal group for families expecting multiples. Given the importance of engaging the mother, father, and couple together, the group program was designed for couples to attend together where possible as well as mothers. The overall principles of the program were to strengthen the social and emotional wellbeing and bond between the parents; to enhance their reflective functioning regarding their transition to parenthood and impact of their own histories; to promote early intervention of mental health problems and to promote maternal and paternal attunement to their babies. Antenatal Group: Eight expectant couples attended the first group, comprising four sessions of two hours. The group was repeated with 5 couples attending. Postnatal Group: Four couples and two mothers attended the postnatal group, comprising four sessions of two hours. A brief overview of the literature on the mental health risk for multiple birth parents will be presented. Group content and pre- and postevaluation data (both qualitative and quantitative) will be presented as well as data on participant risk and those entering individual treatment.

\section{TRIPLETS - TRIPLE COMPLICATION (CASE REPORT)}

N. Vedmedovska ${ }^{1,2}$, A. Miskova ${ }^{1,2}$, and V. Fodina ${ }^{3}$

1.Riga Maternity Hospital, Riga, Latvia

2.Riga Stradins University, Riga, Latvia

3.Ivf Riga, Reproductive Fertility Center, Riga, Latvia

Intoduction: Multiple pregnancies are associated with a high risk of perinatal mortality and morbidity. Among complications are preterm labour, discordant fetal growth and unique for monochorionic pair syndromes. The risk of fetal anomaly is also greater in multiples compared with singleton pregnancy. Ultrasound scanning plays an indispensable role in the diagnosis and management in multiples. Case Report: The objective was to demonstrate the perinatal outcome of triplet pregnancy in 30-year-old patient with a history of infertility. Pregnancy was achieved after intracytoplasmic sperm 
injection (ICSI) and 2 embryo transfer (ET). The I trimester screening ultrasound demonstrated dichorionic triamniotic (BH TA) triple pregnancy with anencephalic fetus A from monochorionic pair. Diagnostic chorionic villus sampling (CVS) was performed and normal karyotype was obtained, but feticide of abnormal fetus was technically impossible. The family decided to postpone the umbilical cord occlusion of fetus A till $16 / 17$ th weeks of gestation. At 18th weeks of gestation twin to twin transfusion syndrome (TTTS) developed. Quintero 3 was confirmed, when anencephalic fetus A was a recipient with polyhydramnion (AF10) and abnormal UA PI, and donor (fetus B) - retarded with an absent end diastolic blood flow (AED). Single fetus $\mathrm{C}$ was appropriate for gestational age and normal doppler flow. Emergency cord occlusion was performed with 3 $\mathrm{L}$ of amniodrainage. The next day donor demise followed as well. Short cervix $(15 \mathrm{~mm})$ was diagnosed on follow up at $23+2$ weeks and Arabin pessary was placed. Premature rupture of membranes was confirmed at $30+0$ weeks. At $31+1$ week of gestation oligohydramnious and distress signs of the survived fetus was the reason for emergency cesarean section. Vaginal delivery was not possible due to presentation of both dead fetuses. Premature boy was born with Apgar score 7/7 at 1 and 5th minutes with a birth weight of $1998 \mathrm{~g}$. At 1 year of age normal development is reported. Conclusion: The antenatal management in multifetal pregnancies poses unique challenges, as the interests of all fetuses have to be taken into account. The timing of intervention thus depends not only on the direct indications, but can be affected/influenced by many other factors, including chances of survival and handicap for each fetus.

\section{TWIN PREGNANCY WITH NEURAL TUBE DEFECTS AND TWIN-TO-TWIN TRANSFUSION SYNDROME: CASE REPORT}

\section{Daşcău' , G. Furău' , C. Furău', C. Onel' , C. Stănescu ${ }^{2}$ and L. Tătaru'}

${ }^{I}$ Department of Obstetrics and Gynecalogy, Vasile Goldiş Western University, Arad, Romania

${ }^{2}$ Department of Anatomy, Vasile Goldiş Western University, Arad, Romania

Introduction: This paper presents the case of a gravida with a 27/28 week twin pregnancy with several complications: neural tube defects, TTTS (a complication seen in monochorionic twin pregnancies) and placenta praevia. We have collected the data from the patient's history and physical examination, as well as from the obstetrical ultrasound and the examination of the fetuses and the placenta and membranes. Results: The patient was admitted to the obstetrics department on June 16, 2011, presenting a minimal vaginal bleeding with red blood; the patient, carrying a twin pregnancy, had been diagnosed two weeks earlier with an anencephalic fetus during an ultrasound scan; the patient had not had a morphofetal ultrasound and she had not had blood tests for fetal anomalies prior to that; her history revealed another birth with an anencephalic fetus on April 1,2005 . The ultrasound performed after the admittance revealed a twin pregnancy with an anencephalic fetus and a fetus with spina bifida, and a twin-to-twin transfusion (TTTS) with a 'stuck twin' syndrome (the anencephalic fetus with polyhydramnios and the other one with severe oligohydramnios) was suspected (there seemed to be an amniotic membrane attached to the fetus with spina bifida). As the bleeding worsened, an emergency $\mathrm{C}$-section was performed and two fetuses were delivered: the first from a transverse lie, weighing $900 \mathrm{~g}$ and presenting a lumbar myelomeningocele and severe oligohydramnios (100-150 ml of amniotic fluid), the second from a cephalic presentation, weighing $700 \mathrm{~g}$ and presenting anencephalia and spina bifida aperta along the entire spine, with polyhydramnios (2000 ml of amniotic fluid); a monochorionic, biamniotic placenta was extracted; The intra and postoperative of the patient was favorable. Conclusions: This case is of high importance because of the association of pathological entities (both twins with neural tube defects and TTTS), as well as because of a history with a prior anencephalic fetus.

\section{TWIN PREGNANCY DISCORDANT FOR SIRENOMELIA:}

\section{CASE REPORT}

\author{
V. Daşcău', G. Furău' , C. Furău' , C. Onel ', C. Stănescu ${ }^{2}$, and L. Tătaru' \\ ${ }^{1}$ Department of Obstetrics and Gynecalogy, Vasile Goldis Western University, Arad, \\ Romania \\ ${ }^{2}$ Department of Anatomy, Vasile Goldiş Western University, Arad, Romania
}

Introduction: This paper presents the case of a 35-year-old gravida with twin pregnancy discordant for sirenomelia (a rare, lethal congenital anomaly characterized by fusion of the lower extremities or a single lower limb, with a number of other abnormalities such as genitourinary and anorectal defects frequently associated and a prevalence of 1-2.5:100,000 in normal pregnancies). We have collected the data from the patient's history and physical examination, as well as from the obstetrical ultrasound and the examination of the fetuses and the placenta and membranes. Results: The patient was admitted in our department on July 5, 2012 at 12:30 pm for premature rupture of membranes; the clinical and ultrasound exams revealed a twin pregnancy with the first fetus in breech presentation and the second in a transverse lie, ruptured membranes (since $11 \mathrm{am}$ ), with cervical effacement and a dilatation of $4 \mathrm{~cm}$. The patient, who had given birth to two healthy newborns, whose ages, birth weights and Apgar scores she was unable to specify, had not had any clinical, ultrasound or paraclinical exams during the pregnancy. An emergency C-section was performed for a 30-weeks' twin pregnancy with both fetuses in non-vertex presentation and PPROM and two newborns were delivered: the first one, in breech presentation, female, weighing $1700 \mathrm{~g}$ and being $45 \mathrm{~cm}$ long, with an Apgar score of 6 and a nuchal cord, and the second one, from a transverse lie, stillborn, with an unspecified sex, weighing $1300 \mathrm{~g}$ and being $43 \mathrm{~cm}$ long, and having sirenomelia; the necropsy of the second newborn revealed several associated malformations: a two lobed right lung, a single lobed left lung, hypoplastic small bowel, megacolon, absent anus, urinary bladder agenesis. The intra and postoperative evolution of the patient and of the first newborn was favorable. Conclusions: This very rare case of a pregnant woman with a twin pregnancy discordant for sirenomelia advocates for a thorough clinical and paraclinical prenatal surveillance of all pregnancies.

\section{CIRCULATING MICRORNA BIOMARKERS AND COGNITIVE FUNCTION IN ELDERLY DANISH TWINS}

Jonas Mengel-From ${ }^{1,2}$, Søren Feddersen ${ }^{3,4}$, Ulrich Halekoh', Niels H. H. Heegaard ${ }^{3,4,5}$, Matt McGue ${ }^{1,6}$, Kaare Christensen ${ }^{1,2,3}$, Qihua Tan ${ }^{1,2}$, and Lene Christiansen

${ }^{I}$ The Danish Aging Research Center and The Danish Twin Registry, Epidemiology, Biostatistics and Biodemography Unit, Department of Public Health, University of Southern Denmark, Odense, Denmark

${ }^{2}$ Department of Clinical Genetics, Odense University Hospital, Odense, Denmark ${ }^{3}$ Department of Clinical Biochemistry and Pharmacology, Odense University Hospital, Odense, Denmark

${ }^{4}$ Department of Clinical Research, University of Southern Denmark, Odense, Denmark ${ }^{5}$ Department of Autoimmunology \& Biomarkers, Statens Serum Institut, Copenhagen, Denmark

${ }^{6}$ Department of Psychology, University of Minnesota, Minneapolis, Minnesota, USA

MicroRNAs (miRNAs) in the blood have been introduced as novel biomarkers of neurodegenerative disease, in particular Alzheimer's disease. MiRNAs are non-coding RNAs typically 19-22 nucleotide bases in length, which regulate protein expression posttranscriptionally and contribute to the epigenetic regulation. Gene regulatory functions by miRNAs occur intracellularly, but miRNAs are also released from a variety of cell types in a protected yet accessible form to the extracellular environment. MiRNAs are both stable and readily detectable as circulating miRNA in plasma, serum and whole blood. We aimed to describe associations between circulating miRNAs and cognitive test performance in elderly twins. Circulating plasma miRNAs were in the present work assayed on a 754 miRNA OpenArray platform using samples from 90 monozygotic elderly twins (73-95 years of age). We performed both within 
twin-pair (controlling for familial factors) and individual level analyses with baseline level of the cognitive performance measured by the mini mental state examination (MMSE) and a five-component cognitive score (CCS). In addition, selected candidate miRNAs were studied further for their association with cognitive decline over 10 years using up to six repeated assessments. A total of $278 \mathrm{miR}$ NAs were successfully detected in plasma from at least ten participants. We found no miRNA significantly associated with CCS and/or MMSE, but several miRNAs were nominally significant associated with the individual cognitive component scores. Generally elderly individuals with poor cognitive function had upregulated miRNAs compared with equivalent individuals who performed better on the cognitive scale. Most notable were three miRNAs, miR151a-3p, miR-212-3p and miR-1274b that associated with CCS, both in the paired and the individual analysis. Also in the longitudinal study we found tendencies that elderly individuals who had upregulated miRNAs were more likely to have a steeper longitudinal decline in CCS with age. Several miRNAs associated with cognitive scores were also promising post-diagnostic biomarkers of dementia, thus we cautiously propose a shared biological path between normative cognitive aging and dementia.

\section{RAPID CERVICAL PHIGFBP-1 TEST IN ASYMPTOMATIC TWIN PREGNANCIES IN PREDICTION OF PRETERM DELIVERY}

Katarzyna Kosinska-Kaczynska, Iwona Szymusik, Dorota Bomba-Opon,

Aleksandra Zygula, and Miroslaw Wielgos

1st Department of Obstetrics and Gynecology Medical University of Warsaw, Poland

Introduction: Phosphorylated IGFBP-1 test is efficient as a predictor of preterm delivery (PTD) in singleton pregnancies. It is especially useful in identifying symptomatic women who will not deliver within 48 hours. The aim of our study was to assess the effectiveness of a rapid second trimester test for cervical phIGFBP-1 in the prediction of spontaneous preterm delivery in asymptomatic twin pregnancies. Material and Methods: A prospective observational study conducted on 232 twin pregnancies tested for phIGFBP-1 at 20 24 weeks of gestation; 151 patients assessed as asymptomatic, with cervical length $>25 \mathrm{~mm}$ at 20-24 weeks were analyzed. The primary outcome was the PTD $<37$ weeks of gestation. Results: The spontaneous PTD occurred in 93 patients $(61.6 \%), 23$ delivered before completing 34 weeks (15.2\%), 9 prior to 32 weeks $(6 \%)$. Patients delivering $<37$ weeks of gestation more often had a positive phIGFBP1 test between 20 and 24 weeks $(p<0.001)$. phIGFBP- 1 had a high positive predictive value (PPV) of 0.82 and moderate negative predictive value $(0.5)$ in prediction of PTD. The positive likelihood ratio (+LR) for PTD was 2.87 (95\% CI: 1.57-5.7). PPV in prediction of delivery $<34$ and $<32$ weeks was low ( 0.16 and 0.14 respectively), while NPV was high ( 0.85 and 0.93 respectively). Conclusions: A test for phIGFBP1 presence might be an additional tool for predicting preterm delivery in twin gestation.

\section{THE TWINS IN PEDAGOGICAL PRACTICE}

\section{Klara Vitkova Rulikova}

Klub dvojcat a vicercat (Twins and Multiplebirth Club), Prague, Czech Republic

During the last six years quantitative research collecting data from twins, parents of twins and teachers of twins has taken place in the Czech Republic. The first phase was done in 2011, collecting data from the above-mentioned groups. The controlled collection of data was done 5 years later in 2016. The data were obtained online through twins clubs' mailing lists, websites for twins and families with twins, and through social networks. The collection of data was done at exactly the same time after five years (i.e., collection time and month of collection). Data collection from the point of view of each group (twins, twins' families, and teachers of twins) were com- pared at both time points. The results are presented in graphs and these are interpreted also in relation to the three groups and time points. The research showed that each group sees the twins differently. Some teachers had not thought about twins as special kind of children, some of them made mistakes that bother twins, some parents did not have the power to fight with school, while others did. It was the first rigorous twin research undertaken in the Czech republic.

\section{PROTON PUMP INHIBITOR USE AND RISK OF COGNITIVE DECLINE: A FOLLOW-UP STUDY OF MIDDLE AGED AND ELDERLY TWINS}

M. Wod' ', J. Hallas ${ }^{2}$, K. Andersen ${ }^{3}$, L. A. García Rodríguez ${ }^{4}$, K. Christensen ${ }^{1,5}$, and D. Gaist ${ }^{6}$

${ }^{1}$ Unit of Epidemiology, Biostatistics, and Biodemography, and the Danish Twin Registry, and the Danish Aging Research Center, Department of Public Health, University of Southern Denmark, Odense, Denmark

${ }^{2}$ Clinical Pharmacology and Pharmacy, Department of Public Health, University of Southern Denmark, Odense, Denmark

${ }^{3}$ Department of Psychiatry - Odense, Region of Southern Denmark

${ }^{4}$ Centro Español Investigatión Farmacoepidemiológica (Ceife), Madrid, Spain ${ }^{5}$ Department of Clinical Genetics and Department of Clinical Biochemistry and Pharmacology, Odense University Hospital, Odense, Denmark

${ }^{6}$ Department of Neurology, Odense University Hospital and Department of Clinical Medicine, Faculty of Health Sciences, University of Southern Denmark, Odense, Denmark

Introduction: Proton pump inhibitors (PPI) are among the most commonly prescribed classes of drugs and their use is increasing. A small number of observational studies suggest that PPI use could affect cognitive function. To further investigate this purported association, we linked information gathered through population-based twin surveys that included repeated assessments of cognitive function with nationwide prescription register data. Apart from the advantages of large sample size and detailed unbiased information on drug use, this approach also enabled us, in subanalyses, to control for familial (genetic and environmental) confounding. Materials and Methods: Data from the Middle Aged Danish Twin study (MADT; baseline and 10-year follow-up) and Longitudinal Study of Danish Twins (LSADT; baseline and 2-year follow-up) combined with national registers including prescription data were used to investigate the possible association between PPI and cognitive decline. In individual-based analyses, twins were stratified according to PPI use and multivariate linear regression was used to test for association between PPI use and cognitive function. Models were adjusted for age, sex, BMI, smoking, alcohol use, physician diagnosed history of depression, neurological disorders (stroke, epilepsy, Parkinson's disease), thyroid disorders, hypertension, diabetes and, based on prescription register data and drugs with a possible direct effect on cognition. Furthermore, intrapair analyses of PPI drug use-discordant twin pairs were performed. Results: A total of 4,994 twins were eligible for follow-up analyses. Compared with the reference group (no PPI use), no statistically significant differences were observed in adjusted cognitive score change in any of the PPI drug exposed groups in middle-aged and elderly twins. In intrapair analyses, we further tested whether the twin with the higher PPI exposure had the largest decline in cognitive score. There were 328 same sex twin pairs discordant for PPI use for the follow-up intra-pair analyses; 104 twin pairs from LSADT with 2-year follow-up and 224 twin pairs with a 10-year follow-up from MADT. For both middle-aged and elderly twin pairs, there was no association between twins having the highest PPI drug exposure and largest decline in cognitive score. Conclusion: In middle-aged and elderly Danish twins PPI use did not influence cognitive function. 


\section{AN IRVING I. GOTTESMAN LEGACY: MONOZYGOTIC AND} DIZYGOTIC TWINS REARED APART

\author{
Nancy L. Segal \\ Department of Psychology, California State University, Fullerton, CA, USA
}

Introduction: In a 2001 interview with Dr. Irving I. Gottesman, published in Twin Research, I posed the question: How should we think of him on the occasion of his retirement? In his usual modest and joking manner, Gottesman conceded that he had no idea that early in his career he was starting an 'avalanche of twin studies,' then discussed his seminal books, papers and projects. Clearly, this prolific investigator left an extensive body of ground-breaking academic research, but he is also famous for the collegiality and support shown to members of this panel and his students and colleagues everywhere. Today, I want to pose the question: How shall we think of Dr. Irving Gottesman in terms of his life-long contributions to twins and twin studies? Materials and Methods: I will address the question above with reference to Gottesman's essays and research on twins reared apart, and my own studies that have been inspired and informed by our many contacts. Among Gottesman's work on separated twins are his introduction to Juel-Nielsen's volume Individual and Environment, a study of Danish MZ twins raised apart, his papers on the Minnesota Study of Twins Reared Apart (MISTRA) and his contributions to my 2012 book, Born Together-Reared Apart, that reported findings from that study. My subsequent research that bears Gottesman's influence includes several paper and case reports of reared-apart and switched-at-birth twins. Results: Dr. Irving I. Gottesman was an extraordinary researcher for whom twin research was a central theme in his psychological and psychiatric studies. In his introduction to Juel-Nielsen's work, he lamented that few people read the original work, as well as the texts of landmark studies on reared-apart twins set in the United States (1937) and United Kingdom (1962). He praised Juel-Nielsen for including life history material that beautifully complemented the quantitative data. Gottesman was also a key player in the MISTRA, authoring studies on the MMPI, intelligence and personality. I felt his influence as I gathered data conducted on reared-apart twins identified after the MISTRA ended. Conclusions: The lifetime achievements of Dr. Irving I. Gottesman will continue to guide many aspects of twin research, not just those mentioned here, but also schizophrenia, depression, and epigenetics. In my possession are several books that he passed on to me as he was reorganizing his personal library, items that are truly treasured.

\section{COMPARISON OF PLANNED CESAREAN DELIVERY AND PLANNED VAGINAL DELIVERY IN WOMEN WITH A TWIN PREGNANCY: A NATION WIDE COHORT STUDY}

Simone Goossens' , Sabine Ensing ${ }^{2}$, Mark van der Hoeven ${ }^{3}$, Frans Roumen ${ }^{4}$, Jan Nijhuis ${ }^{5}$, and Ben $\mathrm{Mol}^{6}$

${ }^{1}$ Department of Obstetrics and Gynecology, Máxima Medical Centre, Veldhoven, the Netherlands

${ }^{2}$ Department of Obstetrics and Gynecology, Amsterdam Medical Centre, Amsterdam, the Netherlands

${ }^{3}$ Department of Neonatology, Maastricht University Medical Centre + , Maastricht, the Netherlands

${ }^{4}$ Department of Obstetrics and Gynecology, Zuyderland Medical Center,

Heerlen-Sittard, the Netherlands

${ }^{5}$ GROW - School for Oncology and Developmental Biology, Maastricht, Department of Obstetrics and Gynecology, Maastricht University Medical Centre +, Maastricht, the Netherlands

${ }^{6}$ The Robinson Institute, School of Pediatrics and Reproductive Health, University of Adelaide, Adelaide, Australia

Introduction: To compare, in women with twin pregnancy with the first twin in cephalic position, neonatal morbidity and mortality rates after planned cesarean delivery (CD) versus planned vaginal delivery (VD). Material and Methods: A nationwide cohort study on women pregnant with twins and the first child in cephalic position, who delivered between $32+0-41+0$ weeks between $2000-2012$ in the Netherlands, using multivariate logistic regression analysis to compare neonatal morbidity and mortality according to planned delivery mode, and looked at subgroups $32+0-36+6$ and $37+0-41+0$ weeks. Results: We included 21,107 women, of whom 1,384 (6.6\%) had a planned CD. Of the 19,723 women (93.4\%) who had a planned VD, $19.7 \%$ delivered by intrapartum CD. We found no significant differences in 'any mortality' (aOR planned CD vs. planned VD 1.34 $(95 \% \mathrm{CI}[0.63,2.60])$, the outcome 'AS $<4$ or death within 28 days' (aOR $1.28(95 \% \mathrm{CI}[0.77,2.11])$ or asphyxia-related morbidity (aOR 0.57 (95\% CI $[0.32,1.03])$. After planned CD more prematurityrelated morbidity (aOR $1.55 ; 95 \%$ CI $[1.21,1.98]$ ) other morbidity (aOR 1.50 (95\% CI 1.26-1.78)) and 'any morbidity or mortality' (aOR $1.4 ; 95 \%$ CI $[1.20,1.66])$ was noted. Trauma-associated morbidity was absent after planned $\mathrm{CD}$ and occurred 45 times $(0.2 \%)$ after planned VD. Before $36+6$ weeks, planned $\mathrm{CD}$ resulted in more perinatal mortality (aOR 2.10; 95\% CI [0.92, 4.76]), while asphyxia-related morbidity did not differ (aOR0.80; 95\% CI [0.41, 1.54]). Planned CD resulted in more 'any morbidity or mortality' (aOR $1.52 ; 95 \%$ CI $[1.25,1.84]$ ), ' $\mathrm{AS}<4$ or death within 28 days' (aOR 1.77 ; $95 \%$ CI $[1.02,3.09])$, prematurity-related morbidity (aOR 1.73 ; $95 \% \mathrm{CI}[1.34,2.23]$ ), and 'other morbidity' (aOR $1.56 ; 95 \%$ CI $[1.28,1.90])$. After 37 weeks, no significance differences in mortality 'any morbidity or mortality <28 days' (aOR0.96; $95 \%$ CI $[0.67,1.38]$ ), or 'AS $<4$ or death within 28 days' (aOR 0.41 ; $95 \%$ CI $[0.10,1.70])$ were found. There was less asphyxia-related morbidity after planned CD (aOR 0.24; 95\% CI [0.06, 1.002]). Conclusions: In women with a cephalic presenting first twin delivering preterm, planned VD results in better neonatal outcomes compared to planned CD. In women at term, there were no significant differences in overall outcomes, with asphyxia related morbidity occurring less often after planned CD.

\section{SINGLE INTRAUTERINE DEATH IN TWINS: THE IMPORTANCE OF FETAL ORDER}

Penelope Ward, Karen Reidy, Ricardo Palma-Dias, Lex Doyle, and Mark Umstad Pregnancy Research Centre and Division of Maternity Services, The Royal Women's Hospital, Melbourne, Australia

Introduction: Perinatal mortality is higher in twin pregnancies. The effect of twin order has not previously been studied in the context of a single fetal demise. Materials and Methods: Multiple pregnancies with two viable fetuses confirmed prior to 20 weeks gestation with the subsequent death of at least one twin were included in this study. All the pregnancies were managed at The Royal Women's Hospital, Melbourne between 2006 and 2014. We excluded pregnancies of higher order, the death of both twins simultaneously and cases with incomplete outcome data. Maternal and neonatal records, discharge summaries, imaging and pathology reports were reviewed. Results: Gestational age at delivery was significantly earlier when the demised twin was presenting $(p<.001)$, and the interval between diagnosis of fetal death and delivery was shorter $(p=.016)$ in these pregnancies. The emergency cesarean rate was also significantly higher, $67 \%$ vs $32 \%(p=.021)$. There was no significant difference between the incidence of chorioamnionitis $(p=$ $.23)$, preterm prelabour rupture of membranes $(p=.66)$ or placental abruption $(p=.08)$. Survival rates were similar for co-twins in both groups $(p=.71)$, but birthweight was significantly lower $(p$ $=.001)$ as were 1 -minute Apgars $(p=.020)$. Death of the leading twin was associated with higher neonatal intensive and special care admission $(p<.01)$, and more respiratory distress $(p<.01)$. Correction for gestational age with regression analysis showed that poor neonatal outcomes were due to the degree of prematurity rather than twin order itself. Separate analysis of dichorionic twins showed significantly earlier delivery when the demised twin was leading, while there was no measurable influence of twin order in monochorionic twins. Conclusions: These results indicate that if the demised twin is presenting, delivery occurs earlier, and morbidity is therefore more 
likely for the survivors. This is especially relevant for dichorionic twin pregnancies.

\section{CASE REPORT OF QUINTUPLET PREGNACY IN UKRAINE}

Svitlana Galych, Svitlana Shevchenko, Vadim Fusun, and Vitaliy Yanak

${ }^{I}$ Odesa Oblast Perinatal Center, Odesa, Ukraine

${ }^{2}$ Odesa Natinal Medical University, Odesa, Ukraine

Introduction: The natural incidence of multiple babies in one pregnancy is: twins are born to one in 83 women. Triplets are born to one in every 7000 women; quadruplets are born to one in 571,000 , quintuplets to one in 47,000,000. Case Report: A 37-year-old woman learned that she would have the five fetuses in the 8th week of pregnancy. This pregnancy was her second, 4 years after the birth of a girl. There were two monochorionic diamniotic twins (two boys and two girls) and one fetus (boy) was alone. We provided careful monitoring. This included more frequent office visits, ultrasounds, fetal non-stress testing and bed rest for the mother. This pregnancy proceeded without complications. At 20 weeks a vaginal pessary was introduced. Only anemia of mild degree was noted, the other data of laboratory analyses were normal. At 28 weeks of pregnancy, ultrasonographic assessment of fetal size confirmed the intrauterine growth restriction of the second twin of girls and moderate polyhydramnios was in the fifth fetus. Corticosteroid medications (dexamethasone $24 \mathrm{mg}$ ) was given to help mature the lungs of the fetus. At 30 weeks of pregnancy, placental abruption occurred in the fifth fetus. Emergency cesarean section was performed by spinal anesthesia. Two live boys were born with weights of $1,810 \mathrm{~g}$ and 1,750 $\mathrm{g}$, two live girls with weights of $1,190 \mathrm{~g}$ and $1,250 \mathrm{~g}$, and the other boy weighed $1,400 \mathrm{~g}$. All children were transferred to the neonatal intensive care department. All the children had respiratory distress syndrome and were given surfactant replacement therapy. A less invasive breathing support option is a nasal continuous positive airway pressure machine given for two boys, while an oxygen mask was used for the other boy. The two girls needed mechanical ventilation for 7-10 days. Following treatment, the children's conditions improved and on the 15th day they were transferred for nursing to the special children's hospital. The children are now 1 year old and are developing normally and are quite healthy. Conclusions: Quintuplet pregnancies are associated with high rates of obstetric complications and the careful monitoring is required.

\section{VARIANT ANATOMY OF THE CIRCLE OF WILLIS: AN MRI} TWIN STUDY

\footnotetext{
B. Forgo', A. D. Tarnoki ',2, D. L. Tarnoki ',2, D. T. Kovacs', A. Persely', A. Hernyes', L. Szalontai' , M. Szily', T. Horvath ${ }^{3}$, A. Sas ${ }^{4}$, M. Sepsi ${ }^{4}$, L. Kostyal ${ }^{4}$, and C. Olah ${ }^{5}$

${ }^{I}$ Department of Radiology, Semmelweis University, Budapest, Hungary

${ }^{2}$ Hungarian Twin Registry, Budapest, Hungary

${ }^{3}$ Department of Hydrodynamic Systems, Budapest University of Technology and Economics, Budapest, Hungary

${ }^{4}$ Department of Radiology, Borsod County University Teaching Hospital, Miskolc, Hungary

${ }^{5}$ Department of Neurology, Borsod County University Teaching Hospital, Miskolc, Hungary
}

Introduction: The Circle of Willis $(\mathrm{CW})$ is the major arterial collateral pathway supplying the brain. Variations of the $\mathrm{CW}$ have been associated with increased risk of cerebrovascular events. Previous studies investigated ethnical differences and genes on animal models but whether these arterial configurations have a genetic background or are determined by environmental factors is not clear. Our aim was to examine the heritability of $\mathrm{CW}$ variants and birth-related factors influencing them. Materials and Methods: 92 adult members of the Hungarian Twin Registry (29 monozygotic [MZ] and 17 dizygotic [DZ] pairs; 29 women, 63 men; average age $49.4 \pm 14.6$ and $51.8 \pm 11.8$, respectively) underwent Time-of-Flight (TOF) MRangiography of the $\mathrm{CW}$. Components of the $\mathrm{CW}$ were examined, hypoplasia and absence of each vessel was determined. Rough her- itability of CW configurations was calculated using the Falconer formula. Perinatal anamnesis was obtained to assess connections between $\mathrm{CW}$ variants and birth-related circumstances. Results: In most of the individuals, normal anterior $\mathrm{CW}$ without hypoplastic or absent vessels was seen (44.57\%). Among posterior CW configurations, bilaterally absent posterior communicating artery was the most commonly seen variant $(23.91 \%)$. Unilateral fetal variant was observed in $10.87 \%$. Bilateral fetal circulation was present in $2.17 \%$. Left absent PCoA occurred significantly more in term-born subjects $(p=.04)$. Right hypoplastic P1 segment of the posterior cerebral artery and right-sided fetal variant was significantly associated with normal birth weight ( $p=.04$ in both cases). Rough heritability of anterior and posterior $\mathrm{CW}$ variants and complete $\mathrm{CW}$ was $0.47,0.43$ and 0.23 , respectively. No or negligible heritability was found regarding hypoplasticity or absence of the vessel components of the $\mathrm{CW}$. Birth weight and gestational age at birth did not differ significantly in MZ twins discordant to anterior and posterior $\mathrm{CW}$ variants. Anterior $\mathrm{CW}$ variants and first-born twins had a borderline significant association $(p=.05)$ in this subgroup. Conclusion: Our results support that $\mathrm{CW}$ variants are mainly determined by environmental factors and do not have a substantial genetic basis. Circumstances and maturity at birth do not influence the formation of $\mathrm{CW}$ variants. Further investigations (including epigenetic studies) are needed to identify exact environmental factors affecting $\mathrm{CW}$ variants which could play an important role in the prevention of cerebrovascular diseases.

\section{DICHORIONIC TWINS PREGNANCY DISCORDANT FOR FETAL ANENCEPHALY}

Selahattin Kumru and Ceyda Etlec

Department of Perinatology, Akdeniz University School of Medicine, Antalya, Turkey

Introduction: Spina bifida and anencephaly, the two most common forms of neural-tube defects, occur in 1 in 1,000 pregnancies. Prenatal detection of anencephaly by ultrasound is possible in almost $100 \%$ of cases. In twin pregnancies discordant for anencephaly, there are potential risks of development of polyhydramnios, preterm delivery and sudden death of one or two fetuses. In this clinical situation, there are three management options: selective fetocide, serial ultrasound examination for polyhydramnios or expectant management. In this paper, we present a case of twin pregnancy discordant for anencephaly which was managed with selective feticide. Case Study: A-27-year-old woman was referred to our department with 13 weeks of twin pregnancy and discordant for anencephaly. An ultrasound scan showed a dichorionic diamniotic twin pregnancy. Twin A's development was concordant to $12+5$ weeks and it was structurally normal but twin B was anencephalic and concordant to 12 weeks. After adequate obstetric and genetic counseling and given all the information about the risks involving this pregnancy, the parents opted for selective feticide. The affected twin has been terminated by ultrasound guided intrathoracic injection of $2.5 \mathrm{~mL}$ potassium chloride $(\mathrm{KCl})$ using a $22 \mathrm{G}$ needle, at 12 weeks. Cardiac assistolie was confirmed at the end of the procedure. The procedure had no early complications. The patient is still pregnant and has reached the 28th week. No complications were observed in the second trimester. Fetal ultrasound measurements and amnion fluid were consistent with the gestational week. Conclusions: Dichorionic, as well as monochorionic twin pregnancies discordant for anencephaly are still requiring an individual approach considering chorionicity/amnionicity, time of gestation and potential risk for pregnancy complications. Selective feticide as early as possible, ideally before 16 weeks, seems to be an acceptable and preferable option for dichorionic twin pregnancies affected by discordance for anencephaly. 


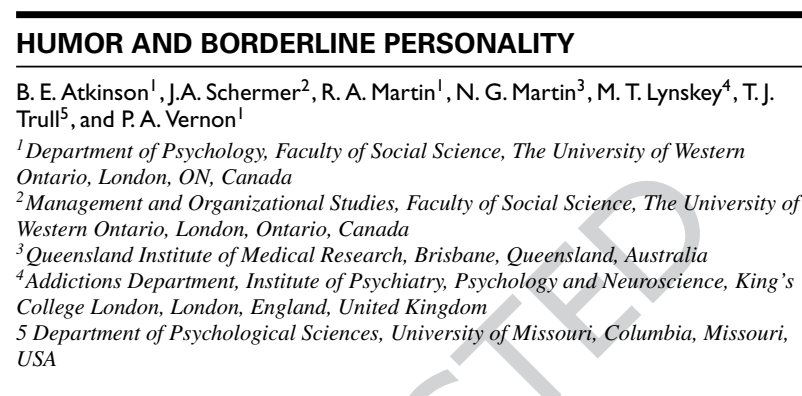

The present study examined the phenotypic, genetic, and environmental correlations between four humor styles (affiliative, selfenhancing, aggressive, and self-defeating) and four dimensions of borderline personality disorder (affective instability, identity disturbance, negative relationships, self-harm) as well as a total borderline personality disorder score. Participants were 574 same-sex Australian adult twin pairs. At the phenotypic level, the two adaptive humor style dimensions (affiliative and self-enhancing) were found to correlate negatively with borderline personality and the two maladaptive humor style dimensions (aggressive and self-defeating) were found to have positive correlations with borderline personality. Bivariate genetic analyses demonstrated significant genetic, common environment, and unique environmental correlations. These results indicate that a large component of the phenotypic association between borderline personality disorder and humor style arises from the influence of correlated genetic and correlated non-shared environmental factors.

\section{A BEHAVIOR GENETIC INVESTIGATION OF THE RELATIONSHIP BETWEEN VOCATIONAL INTERESTS AND MEASURED INTELLIGENCE}

Julie Aitken Schermer' ${ }^{\text {, Philip A. Vernon }}{ }^{2}$, and Andrew M. Johnson ${ }^{3}$

${ }^{I}$ Management and Organizational Studies, The University of Western Ontario, London, Ontario, Canada

${ }^{2}$ Psychology, The University of Western Ontario, London, Ontario, Canada

${ }^{3}$ Faculty of Health Sciences, The University of Western Ontario, London, Ontario, Canada

Although the relationship between personality and vocational interests has received considerable attention, there has been significantly less research directed at the relationship between vocational interests and intelligence. A recent meta-analysis by Pässler, Beinicke, and Hell (2015) suggested that there is a significant phenotypic relationship between some vocational interests and intelligence and this raises the question as to whether these domains share genetic and/or environmental variance. To this end, the relationship between measured intelligence and self-report vocational interest factors was examined in a relatively small archival sample of adult twins as a means of assessing if future, and larger scale, behavior genetic studies should be conducted in this area. Participants completed self-report vocational interest scales which were found to fit a six factor solution. Although not large, the patterns of correlations between the intelligence scales and the vocational interest factors were meaningful. In particular, individuals with investigative interests tended to score higher on intelligence and these phenotypic correlations were also significant at the genetic level. These results suggest that there is some empirical support for a common biological antecedent that predicts both vocational interests and intelligence.

\section{APLASIA CUTIS CONGENITA AS A RESULT OF INTERSTITIAL LASER THERAPY FOR FETAL REDUCTION IN MONOCHORIONIC TWINS: CONSERVATIVE APPROACH AND OUTCOME}

Veronica Mugarab Samedi', Abhay Lodha' ', Adel ElSharkawy' ${ }^{\prime}$ Anar Samedi ${ }^{2}$, and Essa Al Awad'

${ }^{1}$ Division Of Neonatology, Faculty of Pediatrics, University of Calgary, Calgary, Canada

${ }^{2}$ Faculty of Sciences, University of Calgary, Calgary, Canada

Aplasia cutis congenita (ACC) is not a common condition. It occurs with an incidence rate of 1-3 cases per 10,000 live births, regardless of sex or race. Most of the lesions present on the scalp, and congenital skin aplasia on the lower limb is a rare finding. The cause of ACC lesions is heterogeneous. Cases of familial ACC have been described, as well as association with genetic disorders such as Adams-Oliver syndrome or Bart syndrome. The common mechanisms of ACC include vascular disruption, trauma, teratogens, or certain maternal medications during pregnancy (methimazole, azathioprine, valproic acid). Monochorionic (MC) twin pregnancies are known to carry a high risk of twin-to-twin transfusion syndrome (TTTS) that could lead to miscarriage and perinatal death. Demise of one fetus is associated with co-fetal death in $12-25 \%$ of cases. Fetal reduction is the only procedure that could prevent these outcomes. Interstitial laser therapy is commonly used, and considered an effective and safe procedure but it still carries risks for both mother and fetus.Interstitial laser therapy may be associated with ACC in up to $8 \%$ of fetuses. We report ACC in a preterm infant, a survivor of interstitial laser therapy for fetal reduction in MC pregnancy. Case Report: A healthy 27-year-old primigravida mother underwent intrauterine laser ablation for demise of a twin due to severe IUGR, bladder agenesis and reversed diastolic flow secondary to TTTS. Follow-up ultrasound and Doppler studies of the surviving twin revealed no abnormalities.Upon birth at 27 weeks of GA, two small $0.5 \mathrm{~mm}$ lesions were found on the right thigh of the 
infant. A large $(2 \mathrm{~cm}$ by $7 \mathrm{~cm})$ lesion covered with granulated tissue, along with a $1 \mathrm{~cm}$ lesion were seen on the left leg. These lesions were diagnosed as aplasia cutis congenita, a rare potentially serious skin defect involving absence of all skin layers. Dermatology and plastic surgery were consulted; they suggested complete emollient therapy to prevent desiccation of the defect. Gradual epithelization was noted by the end of 6th week of life. Skin lesions healed with no ulcerations or erosions, with formation of a hairless, atrophic scar, which became less prominent with age. The common etiologic mechanisms of ACC in multiple gestational pregnancies are usually related to embolic phenomena due to TTTS. We believe that these lesions are most likely caused by direct burns from the laser therapy.

\section{IMPACT OF A ‘BREECH CLINIC' ON THE MODE OF DELIVERY OF TWIN PREGNANCIES}

Elena Costa, Siham Zaytouni, Valery Bogne, Sara Derisbourg, and Caroline Daelemans

Obstetrics and Gybecology Department, Erasme Hospital, Brussels, Belgium

Introduction: In January 2016 we initiated a specific pathway of care for breech vaginal deliveries (breech clinic [BC]) for a selected population. It significantly increased the chances of vaginal delivery of fetuses in breech presentation at term compared to the years 2010 2015 (OR 3.5, 95\% CI [1.56. 8.03]). Although the intervention is not aimed at twin deliveries we wanted to see if the change in practice indirectly affected their vaginal delivery rate. Materials and Methods: We analysed retrospectively our twin deliveries from Jan 2010 till Dec 2015 ('no BC in place', $n=277$ ) and compared them to those occurred between January 2016 till June 2017 ('BC in place', $n=50$ ). We included twin pregnancies with two live fetuses at initiation of labor or at the moment of planned cesarean section and of 32 gestational weeks or above. We excluded those with double uterine scar and fetal malformation. We compared the proportion of vaginal deliveries in the whole group as well as in the vertex/vertex and the vertex/non-vertex presentation subgroups. We also compared the proportion of adverse neonatal outcomes at the delivery (a composite outcome of either 5-minutes Apgar score below 7, arterial pH below 7, or neonatal death in either one of the twins). Results: The two groups were comparable for maternal age, proportion of primiparous mothers, gestational age and twins' presentation. We found a statistically significant increase of vaginal deliveries in twins in the 'BC in place' group (OR 2.02, 95\% CI [1.08, 3.88], $p=.027)$. The increase was consistent in the vertex/vertex subgroup $(n=122, O R$ $4.1,95 \%$ CI $[1.02,27.46], p=.049)$ and in the vertex/non-vertex subgroup ( $n=78$, OR $1.54,95 \%$ CI $[0.5,5.05])$, although this was not statistically significant in the latter. There was no vaginal delivery attempt if twin A was in breech presentation. There was no statistically significant difference in adverse neonatal outcomes at the moment of delivery between the two groups $(p=.77)$. Conclusions: There was an increase in the proportion of vaginal deliveries for twins after the opening of the $\mathrm{BC}$, without increasing adverse neonatal outcomes at delivery. The limitations of our study are the small number of observations and its retrospective design. Nonetheless we believe that the implementation of a pathway of care for vaginal route for more complex deliveries such as breech presentations might have encouraged practitioners to withstand vaginal deliveries also for twins.

\section{SMOKING DURING PREGNANCY, TELOMERE LENGTH IN PLACENTAL TISSUE, AND INTELLIGENCE DURING CHILDHOOD}

E. M. Bijnens ${ }^{1,2}$, C. Derom ${ }^{2}$, E. Thiery ${ }^{3}$, S. Weyers ${ }^{2}$, and T.S. Nawrot ${ }^{1,4}$

${ }^{1}$ Centre for Environmental Sciences, Hasselt University, Diepenbeek, Belgium

${ }^{2}$ Department of Obstetrics and Gynaecology, Ghent University Hospital, Ghent, Belgium

${ }^{3}$ Department of Neurology, Ghent University Hospital, Ghent, Belgium

${ }^{4}$ Department of Public Health, Leuven University (KU Leuven), Leuven, Belgium

Introduction: Intelligence is influenced by a mixture of genetic and environmental effects. Literature indicates the importance of intrauterine environmental factors on intellectual development. The aim of the study was to investigate maternal smoking during pregnancy and intelligence in childhood and a possible intermediate role of telomere length in placental tissue. Methods: Telomere length was measured in placental tissue of 159 twins (98 pairs) of the East Flanders Prospective Twin Survey. Total, verbal and performance IQ were assessed with the Wechsler Intelligence Scale for ChildrenRevised (WISC-R) in children between age 7 and 15 years old. Data on maternal smoking during pregnancy was collected retrospectively. Results: We show that the relative average $( \pm \mathrm{SE})$ telomere length in placental tissue in mothers who smoked during pregnancy $(0.88 \pm 0.06)$ is significantly $(p=.006)$ lower than the telomere length of non-smokers $(1.08 \pm 0.03)$. However, no association was found between telomere length and IQ. In addition, unadjusted models show a significant association between smoking during pregnancy and intelligence in childhood. But after adjustment for potential confounders, such as parental education, no significant associations were observed. Conclusions: These preliminary results of a potential link between smoking on intelligence in childhood will be further studied in a larger sample size. The association between maternal smoking during pregnancy and telomere length in placental tissue suggests that smoking during pregnancy may have implication on health outcomes later in life, since telomere shortening is associated with age-related diseases such as cardiovascular diseases. This should be elucidated in a future follow-up study.

\section{OBSTETRIC AND PERINATAL OUTCOME OF MULTIPLE PREGNANCIES AFTER IN VITRO FERTILIZATION COMPLICATED BY SEVERE OVARIAN HYPERSTIMULATION SYNDROME}

N. Abián Franco, B. Gastón Moreno, S. García Francés, B. Pérez Munárriz, C. Larrañaga Azcárate, and J. Zabaleta Jurío

Obstetrics and Gynecology Department, Complejo Hospitalario de Navarra, Pamplona, Spain

Introduction: To analyze multiple pregnancy rates and obstetricperinatal outcome of multiple pregnancies after in-vitro fertilization (IVF) and complicated by severe ovarian hyperstimulation syndrome (OHSS). Materials and Methods: A case series study was performed based on 153 severe OHSS patients attending our center from 2003 and 2016. This work includes a comparative review with IVF a non-OHSS patients group, according to singleton or multiple gestation. Several study variables were analyzed, differentiating between singletons and multiple pregnancies: gestation rate, miscarriage rates, gestational age at delivery, obstetric complication rates (threatened abortion, vanishing twin, admission for threatened preterm labor, betamethasone for pulmonary maturation, gestational diabetes, hypertensive disorders of pregnancy, placental abruption, spontaneous or elective induction of labor and vaginal delivery or cesarean section) and perinatal outcome (birthweight, umbilical arterial ph, Apgar score, gender of newborn, admission to neonatal intensive care, congenital malformations and perinatal mortality). Results: A total of 54 gestations occurred in the OHSS group: 29 singletons $(53.7 \%), 23$ twins $(42.6 \%), 2$ triplets $(3.7 \%)$. Obtained results showed higher rates in twins after OHSS group compared to 
non-OHSS IVF twins for: multiple gestation rate (49\% vs. $23 \%, p<$ $.005)$, preterm delivery before 37 weeks ( $78 \%$ vs. $39,7 \%, p=.052)$, low birthweight under $2,500 \mathrm{~g}(78 \%$ vs. $62,1 \%, p<.005)$ and under $1,500 \mathrm{~g}(19.6 \%$ vs. $8,5 \%, p<.005)$, admission to neonatal intensive care (30,2\% vs. $0,5 \%, p<.005)$, female gender (60.5\% vs. $48.4 \%, p$ $<.005)$. There was a lower miscarriage rate in the multiple gestation after OHSS group (5\%) compared to multiple gestation after nonOHSS $(4.1 \%)$. There were no statistically significant differences among these two groups for: gestational age at delivery, threatened abortion, vanishing twin, admission for threatened preterm labor, fetal pulmonary maturation with betamethasone, gestational diabetes, hypertensive disorders of pregnancy, placental abruption, type of delivery, umbilical arterial ph, Apgar score, congenital malformations and perinatal mortality. Conclusions: Multiple gestation rate, as well as prematurity, low birth weight and admission to neonatal intensive care rates in twins and triplets among IVF patients after severe OHSS are significantly higher compared with IVF non-OHSS patients.

\section{SUPERNUMERARY GESTATIONS AFTER DISCORDANT NUMBER OF EMBRYOS TRANSFERRED}

N. Abián Franco, B. Gastón Moreno, B. Pérez Munárriz, S. García Francés, M. Ruiz García, I. Zabaleta Loinaz, C. Larrañaga Azcárate, and J. Zabaleta Jurío

Complejo Hospitalario de Navarra, Navarra, Spain

Introduction: To describe the obstetric outcome of multiple pregnancies conceived after assisted reproductive techniques (ART) resulting final number of fetuses discordant with transferred embryos. Materials and Methods: A case series study was performed based on 8 supernumerary gestations assisted in our center from 2013 to 2016. Four twin gestations took place after single embryo transfer (SET) and four triplet gestations occurred after double embryo transfer (DET). Several study variables were analyzed: cause of sterility, ART, number of transferred embryos, zygosity and chorionicity, miscarriage rates, interruption of pregnancy rates, obstetric complication rates (threatened abortion, vanishing twin, admission for threatened preterm labor, betamethasone for pulmonary maturation, gestational diabetes, arterial hypertension, spontaneous or elective induction of labour and vaginal delivery or cesarean section). Results: A total of 8 gestations resulted to have discordance between the number of final fetuses and transferred embryos occurred in our hospital from 2013 to 2016. Four of them (50\%) were twin pregnancies after one transferred embryo and four $(50 \%)$ were triplet pregnancies after two transferred embrios. ART were perfromed in all cases. Male factor was responsible for sterility in $37.5 \%$ of the cases. Among female sterility cases, endometriosis was identified in $12.5 \%$, premature ovarian failure in $37.5 \%$ and hyperprolactinemia in $12.5 \%$. Vanishing twin was objectified in $50 \%$ of triplet pregnancies. After selective reduction of one of the embryos (two in triplet pregnancy and one in twins), 25\% of gestations resulted triplet, $37.5 \%$ resulted twins and $37.5 \%$ were singleton. First trimester miscarriage rate was $12.5 \%$ and there was one case of second trimester legal interruption of pregnancy. No cases for ovarian hyperstimulation syndrome, gestational diabetes, preeclampsia or cholestasis were observed. Up to $25 \%$ of evolutionary gestations were affected by intrauterine growth restriction. Admission due to threatened preterm labor was necessary in $12.5 \%$ of cases. Vaginal delivery took place in $75 \%$ of twin pregnancies while elective cesarean section was performed in $100 \%$ of triplet pregnancies. Conclusions: Supernumerary gestations are a rare phenomenon after ART. It appears they are not associated with a higher rate of obstetric complications.

\section{MATERNAL AND OBSTETRIC COMPLICATIONS IN TRIPLET PREGNANCIES: SINGLE-CENTER EXPERIENCE}

N. Abián Franco, B. Gastón Moreno, B. Pérez Munárriz, S. García Francés, J. Barrenetxea Arrinda, I. Pedroarena Lecumberri, M. Bazán Legasa, C. Larrañaga Azcárate, and J. Zabaleta Jurío

Complejo Hospitalario de Navarra, Pamplona, Spain

Introduction: To determine maternal complications and obstetric outcome in triplet pregnancies at a teaching hospital. Materials and Methods: A case series study was performed based on 45 triplet gestations attending at our hospital from 2003 to 2016. The data were expressed as percentages and frequencies. The differences in uterine atony according to chorionicity were compared by chi-square test considering a $p$ value less than .05 as statistically significant. $R e$ sults: A total of 45 triplet gestations were recorded, with the overall incidence of 0.7 per 1,000 births $(0.07 \%)$. Up to $83.3 \%$ of pregnancies were trichorionic-triamniotic, and $16.7 \%$ were dichorionictriamniotic. We observed that $85.8 \%$ of pregnancies were conceived after assisted reproductive techniques (28.6\% after artificial insemination and $57.2 \%$ after in vitro fertilization). Maternal average age at delivery was 34 years. Most frequent maternal weight gain subgroup was 10 to $15 \mathrm{~kg}(38.7 \%)$. Up to $18.3 \%$ of patients experienced metrorrhagia, $11.4 \%$ severe preeclampsia and $8.9 \%$ both gestational diabetes and cholestasis. Twin-to-twin transfusion syndrome was found in one case. Up to $15.6 \%$ of pregnancies developed intrauterine growth restriction for one fetus, and $2.2 \%$ for two and all three fetuses (without finding differences statistically significant in terms of chorionicity). Nine patients required urgent cervical cerclage, and in two pregnancies cervical pessaries were used. There were statistically significant differences when postpartum uterine atony was studied in trichorionic (88.6\%) compared to dichorionic group $(57.1 \%), 5.1 \%$ needed blood transfusion and up to $73.3 \%$ of cases were treated with oral iron. Conclusions: An increase in triplet pregnancy incidence has been observed, probably related to rising use of assisted reproductive techniques over time. Chorionicity seems to be related to higher risk of uterine atony. Even so, further studies are necessary in order to conclude a causal association. From our experience, it appears that cervix cerclage may be a valid option in triplet pregnancies with cervix insufficiency that meets selection criteria.

\section{THE SIGNIFICANCE OF YOLK SAC NUMBER IN MONOAMNIOTIC TWINS}

Christine Fenton, Karen Reidy, Marina Demyanenko, Ricardo Palma-Dias, Stephen Cole, and Mark P. Umstad

Royal Women's Hospital, Melbourne, Australia

Introduction: The detection of a single yolk sac on early first trimester ultrasound was once thought to reliably diagnose monochorionic monoamniotic (MCMA) twins prior to determining the intertwin membrane to be absent. This was based on the premise that the yolk sac and amnion split almost simultaneously during embryogenesis so the number of yolk sacs should always equal the number of amnions. In recent years, this concept has been challenged with rare cases reported of monochorionic diamniotic twins with only one yolk sac, and five cases of MCMA twins with two yolk sacs identified on first trimester ultrasound. Materials and Methods: This study reviews our experience with yolk sac number in MCMA twins and its association with adverse pregnancy outcomes. We performed a retrospective cohort analysis of all MCMA twins managed at a tertiary obstetric centre from January 2003 to February 2017. All MCMA twin pregnancies were diagnosed on tertiary ultrasound and, when possible, chorionicity was confirmed on placental histopathology postnatally. MCMA twin pregnancies with accessible first trimester ultrasound images or reports from 5-11' weeks gestation were included in the analysis. Obstetric and perinatal 
outcomes were determined from hospital medical records. Results: 59 MCMA cases were identified over 14 years. 29 cases were included in the analysis, with 19 cases $(66 \%)$ with one yolk sac and 10 $(34 \%)$ with two yolk sacs identified on first trimester scan. Overall, yolk sac number did not correlate with statistically significant differences in pregnancy outcomes. However, some trends were noted: a higher percentage of emergency cesareans ( $42 \%$ vs. $20 \%$ ), gestational diabetes (33\% vs. $0 \%$ ) and higher APGAR scores (1 min 6.8 vs. $5.1,5 \mathrm{~min} 8.4$ vs. 7.7) in the one yolk sac group, and a higher proportion of male fetuses (60\% vs. $8 \%$ ) and fetal anomalies (50\% vs. 26\%) in the two yolk sac group. Conclusions: Two yolk sacs are common in MCMA twin pregnancies, dispelling the original concept that yolk sac number should equal the number of amnions. Yolk sac number was not significantly correlated with any pregnancy outcome or complication. There is a trend towards more gestational diabetes with a single yolk sac and more male fetuses and fetal anomalies with two yolk sacs. This is the largest cohort of MCMA twins in which yolk sac number has been reported. However, further studies with a larger number of pregnancies are necessary to prove or definitely refute these associations. and guidelines, the overall outcome of twin pregnancies may still be improved.

\section{DISCUSSANT AT THE IRV GOTTESMAN MEMORIAL SYMPOSIUM}

\section{Kaare Christensen}

The Danish Twin Registry University of Southern Denmark; Department of Public Health, Odense, Denmark

The highlights from the papers in the Symposium will be summarized, and Irv Gottesman's significant influence on the international twin society will be emphasized, including his central role in twin research in Denmark.

\section{TWIN PREGNANCIES IN FINLAND IN 1987-2014 - TRENDS}

\section{AND CHANGES IN PREGNANCY COMPLICATIONS}

\author{
A-R. Rissanen ', R. Jernman', G. Mika ${ }^{2}$, N. Irmeli ${ }^{3}$, and N. Mika \\ ${ }^{I}$ Obstetrics and Gynecology, University of Helsinki and Helsinki University Hospital, \\ Finland, \\ ${ }^{2}$ National Institute for Health and Welfare, Helsinki, Finland and Karolinska Institute, \\ Stockholm, Sweden \\ ${ }^{3}$ Children's Hospital, University of Helsinki, Finland
}

Introduction: Twin pregnancies carry several risks for both the mother and the offspring. Regular follow-up during pregnancy is required and long-term sequelae may occur especially due to prematurity. In Finland, antenatal follow-up and management is offered by public healthcare and nearly all deliveries take place in public hospitals. No national guidelines, however, exist and hospitals follow their own respective protocols. Our objective was to establish the trends and changes in the incidence and overall outcome of twin pregnancies in Finland during a 28-year study period. With the help of the outline created, it is possible to further improve the outcome of these high-risk pregnancies. Materials and Methods: This extensive register-based study includes all 23,498 twin pregnancies with 46,363 live children born in Finland during 19872014. Data were collected from the national Medical Birth Register and the Care Register (National Institute for Health and Welfare, Finland) regarding the parturient's characteristics, incidences of several pregnancy complications, preterm deliveries, complications in childbirth and perinatal mortality. The data were retrieved by using ICD-9 and ICD-10 codes as well as NOMESCO Classification on Surgical Procedures. The data compiling in Finland is experienced and reliable. Results: Twin pregnancies accounted for 1.4\% of all births in Finland in 1987-2014. Parturients' mean age and body mass index have remained stable, but the shares of over 35 -year-old and obese parturients are growing. The incidences of pre-eclampsia, intrahepatic cholestasis of pregnancy, gestational diabetes, perineal grade I and II tears and postpartum hemorrhage have risen during the study period. On average, $44.9 \%$ of twins were preterm and $27.7 \%$ of twin deliveries were induced. Almost half $(47.1 \%)$ of twins were delivered by cesarean section, in most cases urgently. The reduction in perinatal mortality has been marked during the study period, but remains significantly higher for twin B. Conclusion: Several pregnancy complications showed increasing trends during the study period and nearly half of twins were born preterm or via cesarean section. Obesity and advanced maternal age among twin parturients have risen, enhancing the risks of additive pregnancy complications. With standardized management protocols
BIRTH WEIGHT DISCORDANCE AND LONG TERM OUTCOMES: A SYSTEMATIC REVIEW AND META-ANALYSIS

S. Jahanfar', J. M. Craig' ${ }^{2}$, A. Khalil ${ }^{3}$, and S. Hassan'

${ }^{I}$ Central Michigan University, USA

${ }^{2}$ Murdoch Children's Research Institute, Melbourne, Australia

${ }^{3}$ St George's Hospital, London, UK

Introduction: We aim to study the impact of birth weight discordance (BWD) on discordance for long-term outcomes during childhood and adulthood including all body systems. Materials and Methods: The methodology follows the MOOSE Statement and is explained under six categories: search strategy, databases, study selection, data extraction, quality assessment, and statistical analysis. A comprehensive list of Mesh terms will be attained by three means. First, the definition of each concept will be extracted from MEDLINE. Second, grey literature, conference proceedings, and reference lists of published articles will be explored. Third, specialists in the field will be consulted to identify the Mesh terms. The following databases will be searched, from the earliest available date (mentioned in brackets) to July 2017: MEDLINE (1996), Cochrane Central Register of Controlled Trials (1991), EMBASE (1980), CINAHL (1982). Data extraction and quality assessment forms were designed. Meta-analyses will be conducted using the generic inverse variance with a fixed- or random-effect model in Revman 5.2. The RR, 95\% confidence interval (95\% CI), test statistics for the interaction, and statistical significance of the analysis will be computed. For papers not reporting RR and 95\% CI, the raw data (i.e., number of events and total number of samples in the exposed and unexposed groups) will be used to estimate RR. The heterogeneity across individual studies will be quantified by the I 2 test. Low, moderate, or high degrees of heterogeneity will be approximated by $\mathrm{I} 2$ values of $25 \%, 50 \%$ and $75 \%$, respectively. If the I 2 value was larger than $50 \%$ random-effect model will be used. Reasons for heterogeneity will be investigated by eyeballing extreme RR and sensitivity analysis. Subgroup analyses will be conducted according to the quality score (high/low), zygosity, chorionicity, BWD cut off $(20 \%, 25 \%$ and $30 \%)$, pregnancy complication by twin-to-twin transfusion (yes/no), pregnancy complication by selective fetal growth restriction (BWD $>25 \%$ and when one of the twins is small, that is, has birth weight $<10$ th centile, yes/no), studies adjusted for potential confounders (e.g., yes/no), associated preterm birth $<34$ weeks (yes/no). Results and conclusions will be presented at the conference. 


\section{HERITABILITY OF SUBFERTILITY AMONG DANISH MALE} AND FEMALE TWINS

\author{
L. Juel Ahrenfeldt ${ }^{1,2}$, S. Möller ${ }^{3}$, T. Kold Jensen ${ }^{4,5,6}, K_{\text {. Christensen }}^{2,7}$, and R. \\ Lindahl-Jacobsen ${ }^{1,2}$ \\ ${ }^{I}$ Max-Planck Odense Center on the Biodemography of Aging, University of Southern \\ Denmark, Odense, Denmark \\ ${ }^{2}$ Unit of Epidemiology, Biostatistics and Biodemography, University of Southern \\ Denmark, Odense, Denmark \\ ${ }^{3}$ OPEN - Odense Patient data Explorative Network, Odense University Hospital and \\ Department of Clinical Research, University of Southern Denmark, Odense, Denmark \\ ${ }^{4}$ Department of Environmental Medicine, Institute of Public Health, University of \\ Southern Denmark, Odense, Denmark \\ ${ }^{5}$ Department of Growth and Reproduction and EDMaRC, Rigshospitalet, University of \\ Copenhagen, Copenhagen, Denmark \\ ${ }^{6}$ Hans Christian Andersen Children's Hospital, Odense University Hospital, Odense, \\ Denmark \\ ${ }^{7}$ Department of Clinical Biochemistry and Pharmacology and Department of Clinical \\ Genetics, Odense University Hospital, Odense, Denmark
}

Causes behind low male and female fecundity have been suggested to be initiated in fetal life. However, fecundity is also affected by environmental exposures later in life, and genetic factors are known to play a role. Using survey data on Danish twins, the aim of this study was to quantify the relative contribution of genetic and environmental effects on fecundity, measured as waiting time to the first attempt to achieve a pregnancy. In 1994, a population-based survey was conducted among twins born 1953-1982. Another survey was carried out in 1998 among twins born 1931-1952. We restricted the study population to twins 18 years or older. All analyses were performed both separately for the two surveys and combined. We used a quantitative genetic liability threshold model, splitting the variation of subfertility risk into A (additive genetic effects), D (dominant genetic effects), $\mathrm{C}$ (common environmental effects) and $\mathrm{E}$ (individual environmental effects) and calculated tetrachoric correlations by zygosity. The analyses were performed both crude and univariately adjusted for age at first attempt to become pregnant, smoking, years in school, age at menarge, oral contraceptives and survey. Waiting time was included as a binary outcome, with cut point at 10 months. Based on the Akaike Information Criterion, the model including dominant genetic and unique environmental factors (DE model) gave the best fit in most cases. Adjusted results for all females (746 MZ and $831 \mathrm{DZ}$ twin pairs) showed that the relative contribution of dominant genetic factors to subfertility was $30 \%$ $(95 \%$ CI $[16,44])$ and the remainder $70 \%(95 \%$ CI $[56,84])$ was explained by environmental factors specific for each twin individual, with a tetrachoric correlation of $0.30(0.15-0.43)$ for $\mathrm{MZ}$ and 0.09 (-0.06-0.24) for DZ. For males (531 MZ and $610 \mathrm{DZ}$ twin pairs), the adjusted results showed that dominant genetic factors explained $6 \%(95 \%$ CI $[0,26])$ and the remainder $94 \%(95 \%$ CI $[74,100])$ was due to unique environmental factors, with a tetrachoric correlation of $0.08(-0.12-0.28)$ for $\mathrm{MZ}$ and $-0.04(-0.21-0.14)$ for DZ. All results were consistent across surveys and almost similar for the crude and adjusted models. In conclusion, our results suggest that environmental factors specific for each twin individual explained the largest part (approximately 70\%) of subfertility for females and almost $100 \%$ for males.

\section{ALTERED ANTIBODY RESPONSE TO EPSTEIN-BARR VIRUS IN PATIENTS WITH RHEUMATOID ARTHRITIS AND HEALTHY SUBJECTS PREDISPOSED TO THE DISEASE}

A. J. Svendsen ', M. C. Wulff Westergaard ${ }^{2,3}$, A. Holck Draborg ${ }^{2}$, R. Holst ${ }^{4,5}$, K. O. Kyvik $^{6}$, P. Junker ${ }^{7}$, M. A. Jakobsen ${ }^{8}$, and G. Houen ${ }^{2}$

${ }^{I}$ The Danish Twin Registry, Epidemiology, Institute of Public Health, University of Southern Denmark, Denmark

${ }^{2}$ Department of Autoimmunology and Biomarkers, Statens Serum Institut, Copenhagen, Denmark

${ }^{3}$ Center for Cancer Immune Therapy, Department of Hematology, Herlev Hospital, University of Copenhagen, Denmark

${ }^{4}$ Institute of Regional Health Research, Department of Biostatistics, University of Southern Denmark, Denmark

${ }_{5}^{5}$ Oslo Centre of Biostatistics and Epidemiology, University in Oslo and Oslo University Hospital, Norway

${ }^{6}$ Department of Clinical Research, University of Southern Denmark and Odense Patient data Explorative Network (OPEN), Odense University Hospital, Denmark

${ }^{7}$ Department of Rheumatology, Odense University Hospital, University of Southern Denmark, Denmark

${ }^{8}$ Department of Clinical Immunology, Odense University Hospital, Denmark

Objective: To assess the involvement of Epstein-Barr virus in the pathogenesis of rheumatoid arthritis (RA) and to determine the heritability of antibody responses against the Epstein-Barr virus encoded protein EBNA1. Methods: Three isotypes of EBNA1 antibodies were measured in 137 RA affected and 150 healthy twin pairs. We estimated the effect of RA and RA predisposition, anticitrullinated antibodies (ACPA), IgM rheumatoid factor (RF) on the level of EBNA1 antibodies and the heritability of EBNA1 antibody level and whether the effect of RA on the EBNA1 antibody level was modified by the shared epitope (SE). Results: IgA-EBNA1 antibody levels were higher in twins from RA discordant twin pairs irrespective of RA status and with no effect of ACPA and RF. The IgGEBNA1 antibody level was elevated in healthy co-twins from RA discordant twin pairs but not in RA affected twins. The IgM-EBNA1 antibody level was higher in both RA twins and their healthy cotwins. The effect of RA on the IgA-EBNA1 antibody level was reversed when SE was present. The heritability of IgA-, IgG- and IgM-EBNA1 antibody level was $40.6 \%, 65.5 \%$ and $54.3 \%$, with no effect of environment shared by the twins. Conclusion: EBNA1 antibodies are distinctively different between patients with RA, individuals who are predisposed to RA and healthy subjects. The high level of $\operatorname{IgA}$ antibodies associated with a family predisposition to RA is mainly attributable to genetics and seems to be modified by the shared epitope. Increased IgA-EBNA1 antibody levels may emerge as a risk marker for RA.

\section{ARABIN OBSTETRIC PESSARY TO PREVENT PRETERM DELIVERY IN TWIN PREGNANCIES}

M. Benito Vielba' , A. Agustín Oliva ${ }^{2}$, A. Espiau Romera' ', E. L. Tejero Cabrejas' , C. De Bonrostro Torralba ${ }^{1}$, and J. M. Campillos Maza'

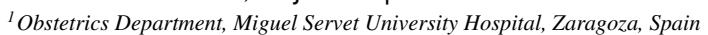
${ }^{2}$ Obstetrics and Gynecology Department, San Jorge Hospital, Huesca, Spain

Introduction: Arabin cervical pessary is a silicone ring for the prevention of preterm birth in pregnant women with short uterine cervix at second trimester. Materials and Methods: We collected data from twin pregnancies assisted at our center from January 2013 to June 2017, analyzing obstetric data, type and gestational age at delivery. The aim of this review was to analyze the gestational results and the prematurity incidence in twin pregnancies treated with obstetric pessary due to a short cervix. Results: During the study period, we assisted 604 twin births. 27.3\% of these pregnancies were achieved spontaneously while the other $72.7 \%$ required assisted reproductive techniques. With respect to chorionicity, $85.2 \%$ were dichorionic-diamniotic and the remaining $14.8 \%$ were monochorionic-diamniotic gestations. We detected 88 cases of short cervix (14.6\%); 83 cases of cervix shortening $<25 \mathrm{~mm}$ before 28 weeks of pregnancy $(13.8 \%)$ and 5 cases of amniotic sac prolapse $(0.8 \%)$. All cases of amniotic sac prolapse were treated by 
an emergency cerclage and it was also performed in seven cases of short cervix. We opted for therapeutic abstention in 12 patients $(13.6 \%)$ and in the remaining 64 cases we opted for an obstetric pessary. All pessaries were inserted between 20 and 28 weeks. With respect to the gestational age at delivery, of all patients treated with pessary, $6.3 \%$ delivered before 28 weeks, $20.2 \%$ between 28 and 34 weeks, $31.3 \%$ between 34 and 37 weeks and $42.2 \%$ achieved a term pregnancy. In the case of patients treated by an emergency cerclage, $41.7 \%$ delivered below 28 weeks; $25 \%$ between 28 and 34 weeks, $25 \%$ between 34 and 37 weeks and only $8.3 \%$ gave birth beyond 37 weeks. In the group of therapeutic abstention, $47.1 \%$ delivered between 28 and 34 weeks, $41.2 \%$ between 34 and 37 weeks and $11.7 \%$ beyond 37 weeks. With respect to the pregnancy termination, $56.6 \%$ of the patients treated with obstetric pessary started labor spontaneously; $6.6 \%$ required oxytocin, $26.3 \%$ required a programmed cesarean section and the remaining $10.5 \%$ needed prostaglandins or double-baloon catheter for labor induction. From the total of patients with cervical shortening, $67 \%$ suffered signs of preterm labor during pregnancy requiring intravenous tocolysis. Conclusion: The obstetric pessary of Arabin could be considered as an alternative to prevent preterm birth in twin pregnancies with a short cervix detected by transvaginal ecography performed during the second trimester.

\section{ARE MONOZYGOTIC TWINS REALLY GENOTYPICALLY IDENTICAL?}

Iwona Szymusik, Katarzyna Kosinska-Kaczynska, Szymon Kozlowski, Robert Brawura Biskupski Samaha

1st Department of Obstetrics and Gynecology, Medical University of Warsaw, Warsaw, Poland

Introduction: Turner syndrome affects approximately 1:2500 liveborn female neonates. More than $99 \%$ of pregnancies with Turner syndome undergo spontaneous miscarriage. $\mathrm{X}$ chromosome monosomy is characterized by fetal edema, nuchal cystic hygroma, heart and kidney abnormalities. Case Reports: Case 1: A 36-year-old woman was referred to the outpatient clinic of the 1st Department of Obstetrics and Gynecology, Medical Univeristy of Warsaw, at 20 weeks of monochorionic diamniotic (MCDA) gestation. At the 13th week, an increased risk of chromosomal abnormalities was diagnosed basing on 1st trimester screening (nuchal translucecny NT I $-17 \mathrm{~mm}$, NT II $-1.3 \mathrm{~mm}$ ). At the 16 th week she underwent amniocentesis of only amniotic sac I. The result revealed X chromosome monosomy of fetus I. Due to a serious prognosis, increasing edema of fetus I (fetus I: $1650 \mathrm{~g}$, fetus II $476 \mathrm{~g}$ at the ultrasound anomaly scan at 21 weeks) and the lack of genetic examination of fetus II, the patient was qualified for the second amniotic sac amniocentesis. It revealed a normal karyotype 46XX. At the 23rd week, intrauterine demise of both fetuses was diagnosed on US scan. The patient was qualified for the labour induction. Case 2: A 22-yearold woman at the 16th week of MCDA twin gestation was referred to the outpatient clinic after amniocentesis. The procedure was performed because of an increased risk of chromosomal abberations of both fetuses, basing on nuchal translucencies (NT I $-1.8 \mathrm{~mm}$; NT II $-11 \mathrm{~mm}$ ). The result of the cytogenetic examination revealed a normal female karyotype of fetus I (46XX) and an abnormal karyotype of fetus II $-45 \mathrm{X} 0$. After the following 3 weeks, the ultrasound examination showed a general edema of fetus II accompanied by the signs typical for twin-to-twin transfusion syndrome (TTTS). The fetoscopic laser ablation of placental anastomoses was performed because of escalating symptoms of fetus I heart failure. Unfortunately, 3 days later intrauterine demise of fetus I was diagnosed. At 21 weeks of gestation the patient decided to terminate the pregnancy, because of the very poor prognosis for edemic fetus II with abnormal karyotype. Conclusions: It was thought that monozygotic twins are genotypically identical. However, in addition to the above, there are cases described in the literature of MC twins with karyotype discordance. The mechanism of an embryo division resulting in an aneuploidy in only one twin is still unclear.

\section{GENETIC AND ENVIRONMENTAL ASSOCIATIONS BETWEEN SELF-EFFICACY, FATIGUE AND DEPRESSION}

F. Inui ${ }^{1,2,3}$, K. Silventoinen ${ }^{2,3}$, C. Honda ${ }^{3}$, R. Tomizawa ${ }^{3,4}$, N. Sakai ${ }^{3,4}$, K. Nakatani ${ }^{1}$, D. Matsumoto', and K. Kato ${ }^{5}$, Osaka Twin Research Group

${ }^{1}$ Faculty of Health Science, Kio University, Nara, Japan

${ }^{2}$ Department of Social Research, University of Helsinki, Helsinki, Finland

${ }^{3}$ Osaka University Center for Twin Research, Osaka University, Osaka, Japan

${ }^{4}$ Osaka University Graduate School of Medicine, Osaka University, Osaka, Japan

${ }^{5}$ Department of Nursing, Kobe City College of Nursing, Hyogo, Japan

Introduction: Self-efficacy is the one of the most important personality characteristics in health promotion and the recovery from or control of disease. However, the background of the relationships between self-efficacy and present somatic and mental symptoms are unclear. In order to clarify these associations, we examined the role of shared genetic and environmental factors underlying the associations between self-efficacy, fatigue symptoms and depressive symptoms. Materials and Methods: Study participants were derived from the Osaka University Twin Registry. The questionnaires were sent to 1,394 twin individuals (697 twin pairs) in 2014, and a total of 720 individuals responded. Because of missing data, 53 individuals were excluded (persons who had incomplete answers for $30 \%$ or more in each scale). A follow-up survey was conducted in 2016. Selfefficacy scores were measured by the General Self Efficacy Scale (GSE), fatigue symptoms by the Chalder Fatigue Scale (CFQ), and depressive symptoms by the Center for Epidemiologic Studies Depression Scale (CES-D). All raw scale scores were standardized for age and sex. Results: When different quad-variate (GSE-2014, CFQ2014, CFQ-2016, and CES-D-2014) genetic models were compared, the additive genetic/specific environment (AE) model offered the best fit according to Akaike Information Criterion (AIC). When four genetic models (AE Cholesky decomposition, AE Cholesky decomposition with two A factors, independent pathway model, and common pathway model) were compared, the Cholesky decomposition with two A components showed the best fit according to AIC. Conclusions: The results show that two different genetic factors underlie the associations between self-efficacy, fatigue, and depressive symptoms. One of the genetic factors contribute to the association between self-efficacy and fatigue symptoms and shows a weak association between self-efficacy and depressive symptoms. Another genetic factor affects fatigue and depressive symptom with equally strength. Our results show that the associations between these traits are largely affected by shared genetic background.

\section{RELATIONSHIP BETWEEN OBSTRUCTIVE SLEEP APNEA} AND LUMBAL DISC PROTRUSION: A TWIN STUDY

Adam Domonkos Tarnoki', Dávid László Tárnoki', Marcell Szily', Dániel Tamás Kovács ', Bianka Forgó' ', Marina B. Pinheiro ${ }^{2}$, Paulo Ferreira ${ }^{2}$, László Kostyál ${ }^{3}$, and Csaba Oláh ${ }^{3}$

${ }^{I}$ Semmelweis University, Department of Radiology, Budapest, Hungary

${ }^{2}$ Arthritis \& Musculoskeletal Research Group, Faculty of Health Sciences, University of Sydney, Australia

${ }^{3}$ Borsod-Abaúj-Zemplén County and University Teaching Hospital, Department of Neurosurgery, Miskolc, Hungary

Introduction: Obstructive sleep apnea (OSA) is a common disease that has been associated with lumbar pain. The literature has pointed to a relationship between sleep disturbance and low back pain, but not a lot of studies have investigated OSA and low back pain. Although literature suggests that sleep disturbance and back pain are closely related, the context of the relationship is still unclear. Our aim was to investigate the relationship between the parameters related to sleep disturbances and the presence of disc abnormalities associated with back pain. Materials and Methods: 74 twin pairs from the Hungarian Twin Registry (52 monozygotic, MZ, and 22 dizygotic, DZ, mean age $51 \pm 15$ years) underwent an overnight 
polysomnography and lumbar spine MRI (Siemens Magnetom Verio 1.5T). Apnoe hypopnea index (AHI), the respiratory disturbance index (RDI) and the oxygen desaturation index (ODI) were registered. In the lumbar spine, the number of disc protusion (bulging) and herniation was recorded per segment. Results: The incidence of OSA, disc protrusion and hernia in the sample was $47 \%, 69 \%$, and $22 \%$ respectively. In patients with OSA, the presence of the disc protrusion was significantly $\left(\chi^{2}=4.117, p=.037\right)$ more frequent. In the case of disc hernia, however, no significant association was detected. The number of disc bulging showed a borderline correlation with AHI $(r=.222, p=.0 .063)$ and a significant correlation with the RDI $(r=.329, p=.007)$. There was no correlation with ODI $(r=.199, p=.097)$. Four twins with OSA of six OSA discordant MZ twin pairs (67\%) had disc protrusion in comparison with the healthy non-OSA brother/sister. We did not find a similar trend for the disc hernias. Conclusions: The presence of OSA is related to the presence of disc protrusion. Further research is needed to examine common genetic factors in the background. The research was supported by the Hungarian Pulmonology Foundation (MPA, 2013).

\section{THE CODATWINS PROJECT: AN EXAMPLE OF} INTERNATIONAL COLLABORATION IN TWIN RESEARCH

\author{
Aline Jelenkovic ${ }^{1,2}$ \\ ${ }^{I}$ University of Helsinki, Helsinki, Finland \\ ${ }^{2}$ University of the Basque Country, Bilbao, Spain
}

Introduction: The CODATwins project (COllaborative project of Development of Anthropometrical measures in Twins) was launched in 2013 with two main objectives: (1) to analyze the variation of heritability estimates of height, BMI and their trajectories over the life course between birth cohorts and geographic-cultural areas, and (2) to study the effects of birth-related factors, education and smoking on these anthropometric traits and whether these effects vary between cohorts. Opportunities to address these questions through international collaboration have improved recently because of both increasing data collection in established cohorts and establishment of new twin cohorts. Materials and Methods: All twin cohorts in the world were contacted and investigators were asked to send individual-level data on height and weight including longitudinal measurements, background variables, birth-related traits, education and smoking to the CODATwins data management center at the University of Helsinki. Results: The CODATwins database is currently the largest twin data set in the world including $>480,000$ twin individuals ( $>225,000$ complete twin pairs) with nearly one million height and BMI measures from 23 countries. Taking advantage of the lessons learnt from this 4-year project, we will discuss issues important for international collaboration in twin research. We will also present the latest status of the database and an overview of the published and ongoing studies. Conclusions: The CODATwins project has demonstrated that large-scale international twin studies obtaining individual-level data are feasible. This project is an example of international collaboration to answer research questions impossible to analyze with a reduced number of cohorts and to extract more information from well-studied traits. Although the focus of this project was restricted to simple traits, the experience obtained may be useful when planning further collaborative projects to analyze more complex issues.

\section{THE BIG 5 OF TWINS' RELATIONSHIPS}

Hila Segal and Ariel Knafo-Noam

Jerusalem Hebrew University, Israel

Introduction: Twin relationships remain understudied despite their influence on individual development and family functioning. Key dimensions in all relationships are closeness and conflict. In addition, growing up constantly together makes dependence, rivalry and dominance especially prominent in the lives of twins. We tested the Twin Relationship Questionnaire (TRQ), a comprehensive measure of twin relationships using mother and father reports on 1,560 twin pairs, tested in five measurement points. Our purpose was to provide a comprehensive psychometric information about the TRQ, so it could be used in the future to investigate relationships between twins. Materials and Methods: Participating children were part of the Longitudinal Israeli Study of Twins (LIST), a study focusing on children's social development as influenced by genetics, abilities, and socialization. Parents completed a questionnaire when the twins were 3, 5, 6.5, 7 (only mothers) and 8-9 years old. Results: At each measurement point we performed exploratory and confirmatory factor analyses on split samples. In addition, multidimensional scaling analysis was performed on a pooled sample combining twins of all ages. The factor analyses yielded five factors: conflict, closeness, dependency, dominance and rivalry, all factors with adequate to high internal consistencies. Correlations between the factors and the multidimensional scaling analysis contrasted the triad of conflict, rivalry and dominance factors as opposed to the dyad of closeness and dependency. Furthermore, a developmental perspective on the correlations between the relationship dimensions suggested interesting patterns. Some of the correlations remain constant throughout the twins' childhood (e.g., correlations between rivalry and other factors), while other correlations change over the years. For example, as twins grow older, twins' attempts to dominate each other increasingly correlate with conflict behavior, regardless of the level of closeness between the twins. Conclusions: The TRQ is a reliable tool that paints a comprehensive picture of the relations between the twins. The replication of the main findings at all measurement points, conducted at different ages and by both parents, further validates the factors that we found. It is our hope that better understanding of twinship by using the TRQ will help shape informed decisions with regards to twins' development.

\section{EPIGENOME-WIDE ANALYSIS OF DNA METHYLATION USING MONOZYGOTIC TWINS DISCORDANT FOR EPILEPSY}

Namitha Mohandas 1,2,3, Yuk Jing Loke ${ }^{1,2}$, Stephanie Hopkins ${ }^{1,2,4}$, Lisa Mackenzie ${ }^{5}$, Carmen Bennett ${ }^{5}$, Sam F Berkovic ${ }^{7}$, Lata Vadlamudi ${ }^{5,6}$, and Jeffrey M Craig ${ }^{1,2,3}$

${ }^{I}$ Murdoch Children's Research Institute, Parkville, Victoria, Australia

${ }^{2}$ Royal Children's Hospital, Parkville, Victoria, Australia

${ }^{3}$ Department of Paediatrics, University of Melbourne, Parkville, Victoria, Australia

${ }^{4}$ University of Newcastle, New South Wales, Australia

${ }^{5}$ Faculty of Medicine, University of Queensland Centre for Clinical Research,

Queensland, Australia

${ }^{6}$ Royal Brisbane and Women's Hospital, Queensland, Australia

${ }^{7}$ Epilepsy Research Centre, University of Melbourne, Austin Health, Victoria, Australia

Introduction: The co-twin control model of disease-discordant monozygotic (MZ) twins enables the study of nonshared environment while controlling for parental factors, location and season of birth, postnatal familial factors, age, ethnicity, sex, and genetics. It has been used to study within-pair epigenetic differences, such as DNA methylation, which have been implicated as both mediators and biomarkers for chronic disorders. Epilepsy is a neurological disorder that affects over 50 million people worldwide. The majority of epilepsies have unknown etiology of presumed genetic origin, and have been termed 'idiopathic'. Epilepsy can be classified into focal (originating from one region of the brain) and generalised (originating bilaterally). The aim of our study was to identify differences in DNA methylation to understand nonshared environmental contributors to idiopathic epilepsies. We also studied whether epilepsy subtypes have distinct epigenetic signatures based on their genomewide methylation patterns. Materials and Methods: Genome-wide DNA methylation analysis was performed within $15 \mathrm{MZ}$ twin pairs discordant for idiopathic epilepsies and between epilepsy subtypes, from DNA extracted from buccal tissue and whole blood, using Illumina's Infinium HumanMethylation EPIC arrays. Data analysis 
was performed using statistical and bioinformatic pipelines. Results: We found 1,000 top-ranked $\mathrm{CpG}$ sites in genes such as calcium and potassium voltage-gated channel genes, $\mathrm{KCNH} 5$ and $\mathrm{CACNB} 2$, where DNA methylation state was significantly different between the subtypes of epilepsy. Analysis of differentially methylated regions (DMRs) identified genes such as PM20D1 and GFPT2, which have a role in neuronal pathways. Separate DNA methylation analysis of discordant focal and generalised epilepsy twin pairs demonstrated top DMR-associated genes OTX1, GDNF and DLX5. Biological functions of these genes support these regions as plausible candidate biomarkers for epilepsy. Conclusions: This is the first epigenetic study to use discordant MZ twins to assess DNA methylation variation in idiopathic epilepsy. The distinct patterns of DNA methylation between subtypes of epilepsy highlight different underlying mechanisms that may account for phenotypic variation. A within twin-pair analysis of focal and generalised epilepsies opens up the possibility of subtype-specific epigenetic marks, which could facilitate biomarker development to assist diagnosis and impact treatment choices in epilepsy.

\section{INTERPERSONAL RELATIONSHIP IN TWINS AND ASSOCIATED FACTORS IN THEIR CHILD CARE}

\author{
Sanae Fukuda' and Yoshie Yokoyama ${ }^{2}$ \\ ${ }^{I}$ Department of Health Welfare Sciences, University of Kansai Welfare Sciences, \\ Kashiwara, Osaka, Japan \\ ${ }^{2}$ Department of Public Nursing, Osaka City University Graduate School of Nursing, \\ Osaka, Japan
}

Background: Mothers of twins showed higher anxiety because of their physical and psychological load. The difficulty of twin care might cause their mothers' having little information, support, economical resource, time, and time of releasing stress. The good interpersonal relationship of twins might be related to the easier child care. However, the interpersonal nature of twin relationships has been little known except from empirical research, and its effects on child care have not been reported. The aim of this study was to examine the factors in mothers with twins associated with interdependent relationships of twins in a community sample. Materials and Methods: This cross-sectional study was conducted using a self-administered questionnaire with mothers in city A. The questionnaire included mothers' demographic variables (e.g., age, employment status, perceived stress, sleep status, self esteem, depression, and gap of twin care) and interpersonal relationships in the twin (e.g., good relationship, synchronicity of behavior, interpersonal interests, and dependent status of the pairs). The study population consisted of 279 mothers with twins aged 0-6 years old who visited a health center in city A. The study was approved by the Ethics Committee of the Osaka City University Graduate School of Nurse and conducted in accordance with the Declaration of Helsinki. All participants consented to participate in the study before enrolment in the document form. Results: The child's age was one of the important factors for appearance of an interpersonal relationship in twins. An interdependent relationship might appear around 3 years old. An interdependent good relationship and synchronicity of behavior of twins influenced their mothers' lower perceived stress compared with relationships that were not. After being divided into two groups, from 0-3 years old (younger group) and 3 years and older (older group), the relationship showed differences by groups. The synchronicity of behavior was associated with mother's stress in the younger group, but the interdependent relationship was associated with mother's stress in the older group. Conclusions: Interpersonal relationship might be an influence on releasing child care stress with twins. However, the association is unclear, and more research is needed.

\section{THE HERITABILITY OF DNA METHYLATION AND LINKS TO HUMAN TRAITS: INSIGHTS FROM TWIN STUDIES}

Jenny van Dongen' , Michel G. Nivard', Gonneke Willemsen', Jouke-Jan Hottenga ', Quinta Helmer', Conor V. Dolan', Erik A. Ehli², Gareth; E. Davies², Maarten van Iterson ${ }^{3}$, Charles Breeze ${ }^{4}$, Stephan Beck ${ }^{4}$, BIOS Consortium ${ }^{5}, \mathrm{H}$. Eka Suchiman ${ }^{3}$, Rick Jansen ${ }^{6}$, Joyce B. van Meurs ${ }^{7}$, Bart M.L. Baselmans ${ }^{1}$, Nuno R. Zilhão', Lannie Ligthart' ${ }^{\prime}$ Meike Bartels' ${ }^{\prime}$, Bastiaan T. Heijmans ${ }^{3}$, P. Eline Slagboom ${ }^{3}$, and Dorret I. Boomsma'

${ }^{I}$ Department of Biological Psychology, VU Amsterdam, Van der Boechorststraat 1, Amsterdam, the Netherlands

${ }^{2}$ Avera Institute for Human Genetics, 3720 W. 69th Street, Sioux Falls, SD, USA

${ }^{3}$ Department of Molecular Epidemiology, Leiden University Medical Center, Leiden, the Netherlands

${ }^{4}$ UCL Cancer Institute, University College London, London, UK

${ }^{5}$ The Biobank-based Integrative Omics Study (BIOS) Consortium

${ }^{6}$ Department of Psychiatry, VU University Medical Center, Amsterdam, the Netherlands

${ }^{7}$ Department of Internal Medicine, Erasmus Medical Center, Rotterdam, the Netherlands

Genetically identical model organisms such as cloned animals, isogenic plants and inbred mice display epigenetic differences. Such organisms, as well as human identical twins, offer insight into the impact of environmental and stochastic influences on the epigenome. The study of DNA methylation in phenotypically discordant monozygotic twins is regarded as a particularly strong research design to identify epigenetic mechanisms involved in human traits and diseases. Epigenetic differences between individuals may also result from genetic variation and here the classical twin design allows estimation of the overall contribution of genetic and environmental factors, from conception onwards. In the classical twin design, this is done by contrasting the correlation between DNA methylation levels of monozygotic (MZ) and dizygotic (DZ) twins, who share $100 \%$ and $50 \%$ of segregating genetic variants respectively. In the first part of this presentation, I will explain how twin studies provide insight into the causes variation between individuals in DNA methylation. I will illustrate this with findings of a large study in which we assessed whole blood genome-wide DNA methylation levels with the Illumina HM450 array in 2,603 individuals from twin families who participate in longitudinal studies of the Netherlands Twin Register. We estimated the total heritability of DNA methylation levels at 411,169 methylation sites and estimated the variance explained by common single nucleotide polymorphisms. Heritability estimates vary across the genome (mean $=$ $19 \%$ ) and we found thousands of sites with sex-specific heritability as well as sites where the environmental variance increases with age. We compared these results with previously published locations where methylation level is associated with metabolic traits, smoking and aging, which illustrated that twin studies provide valuable insight into the genomic locations where methylation variation between people may reflect disease-relevant environmental exposures or genetic variation. In the second part of this presentation, I will discuss how epigenetic studies of monozygotic twins discordant for a trait or disease may give insights into the links between epigenetic mechanisms and traits. I will illustrate this with findings of a study in which we compared whole blood genome-wide DNA methylation levels with the Illumina HM450 array between monozygotic twins discordant for aggressive behavior from the Netherlands Twin Register.

\section{SINGLE PARENTING OF MULTIPLE BIRTH CHILDREN}

J. Susan Griffith

International Council of Multiple Births Organization (ICOMBO)

Purpose: The purpose of this survey done by ICOMBO was to gather information from parents of multiple birth children who reared their multiples as a single parent. The information obtained will be relayed to multiple birth organizations to help them better serve their members who are single parents. Method: A survey 
consisting of 43 questions was distributed via Survey Monkey to single parents of multiples. The survey was distributed mainly by social media (Facebook) and was distributed in seven languages: Czech, English, Finnish, French, German, Japanese, and Spanish. The survey was opened in February 2016 and closed in August 2016. Results: A total of 522 single parents of multiples completed this survey. The majority who responded were mothers but there were also six fathers in this survey. The largest percentage were never married and about $17 \%$ were single by choice (adoption or assisted reproduction). Sixteen countries on five continents were represented in this survey, with Australia, United Kingdom, Finland, United States and France having the most respondents. More than half of the multiples $(61.8 \%)$ were 1 year or younger when their parent became a single parent (some were single by choice though). More than half the respondents said that the non-custodial parent was not active in their childrens' lives. Many of the single parents relied on family and friends for assistance, but some did not get any help at all in the home. Some who were members of a parents of multiples club felt they were treated negatively by the married members of the club, and often club events were planned mainly for couples to attend. Some of the biggest stressors for the single parents were financial problems, keeping up with housework, legal issues, loneliness, worries and fears, feeling inadequate, and difficulty getting out of the house on their own, but the number one issue was lack of sleep and tiredness. Physical assistance in the home was their number one need. Only about a third received regular child support payments and only just over a third did receive financial support from assistance programs. Conclusions: Single parents of multiples face enormous and stressful challenges every day while rearing their children alone. They need the support of family and friends, and they need the support of their local parents of multiples group. They need to be accepted wholeheartedly and their needs should be taken into account when club events and meetings are being planned.

\section{ANTITHROMBOTIC MANAGEMENT AND PERINATAL OUTCOME IN WOMEN WITH MULTIPLE PREGNANCIES AND THROMBOPHILIA:}

B. Grand, M. Arana, L. Voto. Obstetric Team, F. Sobral, M. Horton, S. Papier, G. Leguizamon, S. Kopelman, M. González Alcantara, L. Otaño, M. Sebastiani, A. Trepat, I. Uranga, F. Del Giudice, and F. Neuspiller

Hematology and Laboratory of Thrombosis and Hemostasis Stamboulian, School of Medicine, University of Buenos Aires, Argentina

Introduction: Multiple pregnancies occur either spontaneously or as results of assisted reproduction techniques. The presence of more than one fetus in the womb is associated with increased obstetric and perinatal risk. If additionally the mother has thrombophilia this risk could be higher. Objectives: (1) To provide a comprehensive followup of a selected group of patients with twin and triple pregnancy treated with LMWH for previous detected acquired thrombophilia. (2)To investigate their obstetric and perinatal outcomes. Design: retrospective cohort study. Material and Methods: Medical charts of women referred to the hematological surgery were reviewed. Actual reason of referral was a new pregnancy with antithrombotic management. A total of 18 women were admitted to the hematology surgery: 17 twins and 1 triple pregnancy. Age x $36(28-44)$. No in-vitro fertilization (IVF): 3; with IVF 15 (oocyte donor 5; both gametes donor). Antiphospholipid antibodies (APLA) included: anticardiolipin and anti b2 Glycoprotein I Ig G and Ig M and lupus anticoagulant. Reasons for testing APLA: antiphospholipid syndrome (APS) $n=7$ (with 3 or $>$ recurrent abortions $n=4$; fetal death $n=2$; IUGR $n=1$ ); incomplete APS ( 2 abortions) $n=4$; occasional APLA ( 1 abortion and/or IVF implantation failure) $n=7$. Treatment: enoxaparin $40 \mathrm{mg} / \mathrm{d}$ (15); $60 \mathrm{mg} / \mathrm{d}$ (3) during whole pregnancy and postpartum for venous thromboembolism (VTE) prophylaxis. Results: Early abortion $n=3$; cesarean section $n=15$; mean twins' newborn weight: $2,322 \mathrm{~g}(1,700-3,200 \mathrm{~g})$; triple $(1,750 \mathrm{~g}, 1,350 \mathrm{~g}, 1,350 \mathrm{~g})$.
Gestational diabetes: $n=3$; preeclampsia $n=1$ (triple gestation); premature membranes rupture (PMR) $n=2$; IUGR $n=1$. There are three ongoing pregnancies. Main outcome measure(S): Duration of neonatal admissions 3-28 days. No newborn death. All are actually alive. Other complications: viral infection $n=1$; pneumothorax $n=1$. Maternal complications: post-partum hemorrhage $n=1$. Comments and Conclusions: This selected population of women with twin and triple gestations, most of them conceived following IVF treatment, were treated with prophylactic doses of enoxaparin because of the detection of APLA. All delivered by cesarean sections and there were few perinatal complications. IUGR was presented in women with APS and preterm delivery was associated with (PRM); only the triple pregnancy developed preeclampsia. No hemorrhagic neither thrombotic complications were observed.

\section{ULTRASOUND DETECTION OF PROXIMATE UMBILICAL CORD INSERTIONS (PCIS) IN MONOCHORIONIC TWIN PREGNANCIES. CASE STUDY}

Tamara A. Yarygina' ', Anna A. Klassen ${ }^{2}$, Elena V. Trifonova ${ }^{3}$, and Roza S. Bataeva ${ }^{1,4}$

${ }^{1}$ Fetal Medicine Centre MEDICA, Moscow, Russia

${ }^{2}$ Perinatal center, Orenburg, Russia

${ }^{3}$ Medical Centre Hippocrates, Moscow, Russia

${ }^{4}$ Russian Medical Academy for Advanced Study, Moscow, Russia

Introduction: Proximate cord insertions (PCIs) is one of the abnormal umbilical cord insertion in monochorionic (MC) twins, when the distance between the cord insertions is less than $<4 \mathrm{~cm}$. PCI in $\mathrm{MC}$ diamniotic (DC) placentas is quite rare whereas in $\mathrm{MC}$ monoamniotic (MA) placentas it is quite common. We present a series of four cases of monochorionic (MC) twins with diamniotic and monoamniotic placentas with PCIs. Case Report: Case 1: A 26-year-old woman (G2P0) with MCDA pregnancy by spontaneous conception. US scan at $12 \mathrm{w}$ identified PCIs. The umbilical cords were inserted side by side with one anastomosing vessel between them. The woman was delivered at $36+5$ weeks due to severe oligohydramnios of the 2 nd twin. Birth weights of 1 st and 2 nd twin were $2,600 \mathrm{~g}$ and 2,400 $\mathrm{g}$ respectively with a $7 \%$ discrepancy. Apgar score was $8 / 8$ and $8 / 8$ in 1 and 5 minutes. Case 2: A 45-year-old woman (G1P0) with MCDA pregnancy after IVF. US scan at 12 weeks identified PCIs with superficial anastomoses. The distance between the cords was equal $1.5 \mathrm{sm}$. The woman was delivered at 34 weeks due PROM. Birth weights of 1st and 2nd twin were 2,910 $\mathrm{g}$ and 2,890 g respectively with only a $0.4 \%$ discrepancy. Apgar score was $7 / 8$ and 7/8 in 1 and 5 minutes. Case 3: A 31-year-old woman (G6P2) with MCMA twin pregnancy by spontaneous conception. We identified PCIs and the cord entanglement with the cord knots at the first scan in our clinic at 28 weeks. The distance between the cords was equal $2.8 \mathrm{sm}$. The woman underwent a cesarean delivery at 38 weeks. Birth weight of 1 st and 2 nd twin was 3,100 $\mathrm{g}$ and 3,470 $\mathrm{g}$ respectively with an $11 \%$ discrepancy. Apgar score was 8/9 and 9/9 in 1 and 5 minutes. Case 4: A 37-year-old woman (G6P3) with MCMA twin pregnancy by spontaneous conception. The cord entanglement and PCIs were visualized at 34 weeks' scan, when the patient was admitted to our clinic. The umbilical cords were inserted side by side. The woman underwent a cesarean delivery at 34 weeks due to distress of 2 nd twin. Birth weight of 1 st and 2 nd twin was 2,000 g and $1,800 \mathrm{~g}$ respectively with a $10 \%$ discrepancy. Apgar score was $7 / 7$ and $7 / 7$ in 1 and 5 minutes. Conclusions: Proximate cord insertions were identified correctly by US scan at different gestational ages and confirmed during placental examination. There were neither signs of TTTS, nor of selective IUGR, and there was a favorable outcome in all these cases. 
THE RELATIONSHIP OF PHYSICAL HEATH TO MENTAL HEALTH IN THE VIETNAM ERA TWIN STUDY OF AGING (VETSA)

Michael Lyons' , Mark Logue', Ruth McKenzie' , Rosemary Toomey', Matt Panizzon ${ }^{2}$, Carol Franz ${ }^{2}$, and William Kremen ${ }^{2}$

${ }^{I}$ Boston University, Boston, USA

${ }^{2}$ University of California, San Diego, La Jolla, USA

Introduction: A simplistic dualism distinguishing physical health from mental health is inconsistent with our current understanding of human biology, but treating these domains as separate is a convenient heuristic. Materials and Methods: Data were collected from approximately 1,000 participants in the Vietnam Era Twin Study of Aging (VETSA). The sample comprises men who served in the military between 1965 and 1975 . VETSA includes data on major depression and posttraumatic stress disorder (PTSD) that were collected when the average age of participants was 41 years. During the second wave of VETSA data collection subjects were 62 years old on average and completed the Center for Epidemiologic Studies Depression Scale, the self-report DSM-IV based PTSD Checklist Civilian Version, and self-report questions about medical conditions. Results: Cross-sectionally at age 62, symptoms of depression and PTSD were associated with self-reported sleep apnea, hypertension, diabetes, migraines, hypercholesteremia, gastritis, osteoarthritis, rheumatoid arthritis, and erectile dysfunction. Depression, but not PTSD, was associated with asthma. PTSD, but not depression, was associated with heart disease. Symptoms of depression and PTSD were associated with self-rated general health and lifesatisfaction at age 62 . In longitudinal analyses examining the association of depression at age 41 with health outcomes at age 62 , we controlled for depression at age 62. Similarly, analyses of age 41 PTSD controlled for age 62 PTSD symptoms. Symptoms of depression at age 41 were associated with self-reported stomach ulcer and gastroenteritis at age 62. Symptoms of both depression and PTSD at age 41 were associated with erectile dysfunction at age 62. Symptoms of depression and PTSD at age 41 were also associated with elevated C-reactive protein at age 62 after controlling age 62 depression and PTSD. Symptoms of depression and PTSD at age 41 were not associated with age 62 self-rated general health and life-satisfaction when age 62 PTSD and depression were controlled. Conclusions: As might be expected, there were more significant cross-sectional relationships between medical disorders with depression and PTSD at age 62 than longitudinal relationships. However, a number of age 62 health outcomes were related to psychopathology at age 41 even after controlling for psychopathology at age 62, indicating an association that persisted for over two decades.

\section{THE EVALUATION OF DISCORDANCE IN TWINS WITH SPECIFIC AND NON-SPECIFIC PATHOLOGY BY MEASUREMENTS OF LIVER, THYROID GLAND AND KIDNEYS}

Iryna Gordienko, Olena Tarapurova, Ganna Grebinichenko, and Andriy Velichko Department of Fetal Medicine, Institute of Pediatrics, Obstetrics \& Gynecology of National Academy of Medical Sciences of Ukraine, Kyiv, Ukraine

Introduction: Discordant biometric indices in twins may be the markers of pathologic development. Prenatal ultrasound of some organs is important for establishing complete diagnosis. Objective is to propose the new approaches to the evaluation of well-being of twins with discordance. Materials and Methods: In fetuses with specific and non-specific complications of 58 monochorionic diamniotic (MCDA) twin pregnancies, the following investigations were performed: 2D/3D ultrasound, Doppler, and invasive procedures on indication. The following parameters in twins were analyzed: expected fetus weight, amount of amniotic fluid, measurements of liver, kidneys and thyroid gland. Results: The research showed that the maximum discordance of all studied parameters took place at feto-fetal transfusion syndrome (FFTS). The presence of significant discordance was noted for the expected fetus weight in 2 nd and 3rd trimester $(31.7 \% \pm 2,9)$, amount of amniotic fluid $(76.2 \% \pm 3,36)$, size of the liver $(27.5 \% \pm 2,8)$ and thyroid gland $(34.7 \% \pm 2,35)$. Maximal discordance was seen in the amount of amniotic fluid which is a typical manifestation of pathology. In selective intrauterine growth retardation (SIUGR), there was significantly high discordance between fetuses in relation to the expected fetus weight $(32.4 \% \pm 3,36)$ and amount of amniotic fluid $(38.8 \% \pm 4,2)$, compared to the control group. The higher discordance of the amount of amniotic fluid is associated with oligohydramnion, which occurs in $33.3 \%$ of SIUGR. Since there was no polyhydramnion in the larger fetus, there was no discordance of the amount of amniotic fluid. Conclusions: The discordance of the expected fetus weight, the amount of amniotic fluid, the size of the liver, kidneys and thyroid gland in fetuses with specific and nonspecific complications of multiple pregnancy was evaluated. The discordance may be diagnosed not only with expected fetus weight, but also by measurement of only liver or thyroid gland.

\section{HELLP SYNDROME WITH MONOCHORIONIC GEMELLA: CASE REPORT}

Sonja Srzentic, Vesna Djokvucic, and Zoran Srzentic

Gynecology and Obstetrics Department, Jzu Opšta Bolnica (Phi General Hospital) Bar, Montenegro

Introduction: The number of twin pregnancies has increased over the last few decades and reaches $3 \%$ of all deliveries thanks to assisted fertilization. The mode of delivery depends on the existent complications in pregnancy. The birth itself is much often complicated even by placenta abruption, by placenta and vasa praevia, by premature rupture of membranes, by preterm delivery and prematurity, by abnormal presentation, collision, prolapsed of the umbilical cord, by uterine dysfunction, by hypotonia and postpartum hemorrhage. The optimal time to complete uncomplicated twins pregnancy at diorionic gemellas is the 38 th gestational week. In monohorionic gemella, it is necessary to finish pregnancy after the 34th gestational week, or the 37th gestational week at the latest. In monoamine twins pregnancy, there is a high risk of twisting of umbilical cord, so it is recommended to end pregnancy between 32nd and 34th gestational week after corticosteroid therapy. Case Report: A patient aged 30 years, primipara in 36 th $/ 37$ th gestational week. She denied previous illness, TA 140/100. She had been hospitalized due to fatigue and swollen feet. At the patient laboratory, clinical and ultrasound examination were performed, and it wAs concluded that it was a vital gemellar pregnancy 36th/37th gestational week (MH, DA). Biometrics and morphology of the fetus were neat. CTG records were reactive. Laboratory findings of April 7, 2017 showed WBC 9.28, RBC 3.65, Hgb 92, HCT 261, AST 470, ALT 389, CRP 13.2. The findings on April 9, 2017 showed CRP 21.3, AST 1084, ALT 1005, PLT 229. On April 9, 2017, delivery of 2 male children was performed by cesarean section: 1 st male child 3,110/52/34 APGAR 9/9; 2nd male child 2,700/50/33 APGAR 9/10. Puerperium started out neatly. The patient was released on the 4 th postoperative day in good general condition. Laboratory findings are of the reference values at discharge. Conclusion: Preeclampsia occurrs in $10 \%$ of pregnant women and is the leading cause of maternal and fetal morbidity. Increased pressure in pregnancy and the occurrence of HELLP syndrome was caused by an increase of placenta blood vessels development with placenta ischemia. HELLP syndrome is often overlooked and can be diagnosed as hepatitis. In this case, HELLP syndrome in a pregnant woman with monohormone gemella was diagnosed timely and the pregnancy worked out favorably. 


\section{THE UMBILICAL CORD PATHOLOGY - THROMBOSIS OF} THE UMBILICAL CORD: CASE REPORT

Vesna Djokvucic, Sonja Srzentic, and Zoran Srzentic

Gynecology and Obstetrics Department, Jzu Opšta Bolnica (Phi General Hospital) Bar, Montenegro

Introduction: The umbilical cord (funiculus umbilici) is a connection between the mother and the fetus and consists of one vein and two arteries. Its average length is about $55-60 \mathrm{~cm}$, but it can be up to 3 meters long. A gynecologist-obstetrician, during delivery, often comes across a vast variety of umbilical cord pathology. These states can be divided into two groups: (1) organic disorders - umbilical insertion disorders, vasa previa, umbilical blood vessels pathology, umbilical hernia and tumors of umbilical cord; and (2) functional disorders - true and false knot of umbilical cord, strangulation by the umbilical cord around the neck, umbilical cord descent, umbilical cord prolapsed, umbilical cord torsion, umbilical cord hematoma, umbilical cord thrombosis and umbilical cord edema. Umbilical venous thrombosis occurs rarely and it has an unpredictable prognosis. The incidence of reporting is 2.5-4.5/10,000. It hits more commonly the female sex - 1.6:1. Case Report: The patient D.S., aged 28, quartipara was hospitalized due to pain in the bottom of her stomach and notable edema in her feet. Clinical and ultrasound treatment of the patient was performed, and it was concluded that it was a vital pregnancy in the 39th gestational week. Biometrics and morphology of fetus were in good order. The CTG record was reactive. On the third day of hospitalization, the patient complained that she did not feel the movements of the fetus. The CTG record was silent, and an emergency cesarean section was conducted. A female baby was born live and reanimated. The amniotic fluid was green. The umbilical cord was thrombosed. The placenta measured $15 \times 13 \times 4 \mathrm{~cm}$ with cord length of $45 \mathrm{~cm}$ and a peripheral insertion of umbilical cord that was twisted. Cross-sectional, in luminal blood vessels, there were thrombotic masses (Dg: thrombosis venae umbilicalis. Oedema villorum placentae cum haemorrhagia). Conclusion: The presented case of a patient with umbilical venous thrombosis who gave birth to live female child thanks to the rapid cesarean section. Intrauterine diagnosis of umbilical venous thrombosis is difficult. The umbilical venous thrombosis rarely occurs and its prognosis is unpredictable, fatal in most cases. Maternal and fetal pathology is missing in many cases. It occurs suddenly and its loss is a tragic one. The diagnosis is often set out after delivery, based on histopathology of umbilical cord.

\section{THE ELIZABETH BRYAN MULTIPLE BIRTHS CENTRE \\ Merryl Harvey ${ }^{1,2}$, Jane Denton ${ }^{2,3}$, Louisa Clifford' ', and Laura McGrath-Scott' \\ ${ }^{1}$ Faculty of Health, Education and Life Sciences, Birmingham City University, Birmingham, $U K$ \\ ${ }^{2}$ Elizabeth Bryan Multiple Births Centre, Birmingham, UK \\ ${ }^{3}$ Multiple Births Foundation}

Introduction: The incidence of multiple births in the United Kingdom continues to rise (National Institute for Health and Clinical Excellence, 2011) with the number of multiple maternities increasing from 11.6 per 1,000 in 1990 to 16.1 per 1,000 in 2015 (ONS, 2016). While the NICE Clinical Guideline 129 (NICE, 2011) focused on improving maternity and perinatal care, there are currently no evidence-based guidelines for health care professionals regarding the provision of more long-term care and support for families with twins and higher multiples. To address this need, The Elizabeth Bryan Multiple Births Centre (EBMBC) has been established at Birmingham City University (BCU) and is a collaboration between the Multiple Births Foundation (MBF) and BCU. The core aims of the EBMBC are to: develop evidence-based education and professional development programs for health care and allied professionals involved in the care of multiple birth families; establish standards for education and professional development for health care and al- lied professionals; establish a dedicated research program that will provide an evidence base for practice; support the provision of best possible care for babies, children and their families; collaborate with families to inform education, practice and research. Materials and Methods: The EBMBC was established in April 2017 and is co-led by Dr Merryl Harvey (BCU) and Jane Denton (MBF). A program of research is being developed and will include collaborations with external partners. Graduate research teaching assistants are currently undertaking $\mathrm{PhDs}$ on various issues relating to multiple births. Results: An online module, 'The individualised care of multiple birth families from conception to one year', for undergraduate and postgraduate students will be delivered 2017-2018 at levels 6 and 7. It is anticipated this will be the first of a suite of modules for a range of health and allied professional groups. A study of health visitor experiences supporting multiple births families is in progress. Conclusions: It is anticipated that the EBMBC will support the provision of the best possible care for babies, children, parents and their families.

\section{A TWIN STUDY OF THE COMMON GENETIC AND ENVIRONMENTAL INFLUENCES BETWEEN MOTOR CONTROL AND ADHD SYMPTOMS}

Anna Ogliari ${ }^{2}$, Corrado Fagnani' ${ }^{1}$ Elettra Pezzica ${ }^{2}$, Raffaella Belotti ${ }^{2}$, Emanuele Medda ', Gabriel Baud-Bovy ${ }^{2}$, Marco Battaglia ${ }^{2}$, and Maria Antonietta Stazi' ${ }^{I}$ ISS, Rome, Italy

${ }^{2}$ Child \& Youth Psychiatry, Centre for Addiction and Mental Health, Toronto, Canada

Introduction: In the current study, visuo-motor performances, evaluated through a haptic interface, and scores of ADHD symptoms, tested by questionnaires, have been analyzed in a sample of young twins from the general population. The study was aimed at (1) measuring genetic and enviromental contributes related to individual variance of visuo-motor performances inaccuracy and ADHD symptoms by way of univariate method, and (2) examining cooccurrence casual source between ADHD symptoms and a particular motor control performance by bivariate approach. Materials and Methods: A total of 99 complete pairs aged 6-17 years from the Italian Twin Registry underwent a different motor task, while their parents completed a behavioral questionnaires. A haptic interface was used to deliver a force and record the hand trajectory. Every participant completed the same experimental design, which included several different motor tasks. Univariate and bivariate analyses with MX were then applied to psychometric and motor behavioral data. Results: The analysis results disclosed: (1) how enviromental factors are of prime importance to the motor tasks under observation, whereas the additional genetic contributes are of prime importance to ADHD symptoms; and (2) how the co-occurance between the choosen visuo-motor task and ADHD symptom scale is small but significant, and how that occurance is explained both by genetic and enviromental factors. It also points out that from one side the additional genetic factors, which are responsible for cooccurance between the traits, are widely the same in the two phenotypes, while the genetic enviromental contributions are almost entirely separated. Conclusion: Comparing our results to the preceding study, it emerges that both of them have found a weak but significant correlation between the examined motor task and ADHD symptoms, and that the link between these two variables is mainly explained by the common genetic component. In addition, our study found a sizeable overlap between genetic additive factors explaining the correlation between the traits considered. Starting from the results here reported, our research suggests that future study could be carried out using larger samples and more specific tasks in order to examine the co-occurence among the visuo-motor skills having tighter references with ADHD traits. 
THE RELATIVELY RECENT SOUTHERN EU EXPERIENCE - A DECADE OF TWIN RESEARCH IN SPAIN

\author{
Juan R. Ordoñana \\ University of Murcia and Murcia Institute for BioHealth Research, Spain
}

The first data from a large twin sample in Spain were collected a decade ago, in 2007, by the Murcia Twin Register (MTR). Putting aside previous isolated and limited attempts, this was the first structured initiative to incorporate Spanish science to the twin research community. During this decade the MTR has grown up as a population-based registry, experiencing both success and failure. Our main concerns as an incipient register were know-how and funding; however, there is much more to establishing a twin register. Based on this knowledge we identify some critical points that, obvious as they appear, still present some peculiarities that need to be highlighted. Therefore, their recollection can be of help in the first steps of registry development: (1) Clarify ideas (Do we really need a twin register? And, what for?); (2) Refine a concise research agenda (What are the main topics to study? Can our new registry make any meaningful/interesting contribution to scientific knowledge in the short-term?); (3) Look for synergies (Who is doing what in my area of interest? - In and out of the twin research community); (4) Identify basic human resources (Who is available and willing?) and training opportunities for them (Courses and workshops); (5) Have parallel plans according to different levels of resources (Can we adapt to different environments?); (6) Define recruitment tactics (Who? Where? How?); (7) Plan first funding opportunity (Agency? Are we competitive enough?). Key transversal elements present throughout these points are strategic planning of research objectives, optimization of available resources, and best practice benchmarking.

\section{A GENOME WIDE ASSOCIATION STUDY IDENTIFYING A GENETIC VARIANT FOR FRAILTY USING THE TWINSUK COHORT}

Serena Verdi ', Rosa Cheesman', Ruth Ce Bowyer', Massimo Mangino', Timothy D. Spector' ${ }^{\prime}$, and Claire J Steves ${ }^{1,2}$

${ }^{I}$ Department of Twin Research and Genetic Epidemiology, King's College London, London, UK

${ }^{2}$ Clinical Age Research Unit, Department of Clinical Gerontology, King's College Hospital, London, UK

Introduction: Frailty is commonly defined as poor resilience to external stressors, resulting from aging-related declines across numerous physiological systems, causing vulnerability to adverse outcomes. A study in the TwinsUK cohort suggests a genetic basis of frailty with a reported heritability of $39 \%$ in the Fried Frailty Phenotype (FP). However, a limited number of genes for frailty have been examined in small cohorts with no genetic variant consistently found to be associated. An approach using multiple large cohorts will improve understanding of the genetic underpinnings of frailty. Our study contributes to a meta-Genome Wide Association Study (GWAS) for Cohorts for Heart and Aging Research in Genomic Epidemiology (CHARGE). Materials and Methods: Using the TwinsUK cohort we investigated potential genetic variants associated with frailty in 753 mostly female $(90 \%)$ individuals over 65 . GWAS was implemented using an ordinal 5-point FP scale (comprising of grip strength, gait speed, exhaustion, physical activity, and weight loss) and a newly proposed FP continuous frailty scale. The GWAS included covariants of principle components, age and sex. SNPs were excluded at MAF0.95. Results: The ordinal 5-point scale GWAS showed a significant association (assumes genomewide significance at $p$ value $<5 \times 10-8$ ), $p$ value: $8.02 \times 10-09$ for two variants within the Mannose Receptor C-Type 1 (MRC1) and Transmembrane Protein 236 (TMEM236) gene at chromosome 10. The continuous scale GWAS showed a significant association for an unknown variant on chromosome 2 SNP rs115385423, $p$ value: $6.38 \times 10-09$.
Conclusions: Previous studies indicate MRC1 and TMEM236 have involvement with metabolic pathways governing immune response and body weight respectively; these etiologies can contribute to the complex frailty phenotype. However, our associations were not replicated in the continuous scale GWAS, stressing the importance to validate findings using a larger scale meta-GWAS. Further analysis across multiple cohorts will therefore increase our understanding of the genetic underpinnings of frailty.

\section{COGNITIVE PERFORMANCE IS ASSOCIATED WITH THE GUT MICROBIOTA OF COMMUNITY DWELLING AGEING ADULTS}

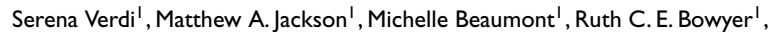
Jordana T. Bell', Tim D. Spector' ${ }^{\prime}$, and Claire J. Steves ${ }^{1,2}$

${ }^{1}$ Department of Twin Research and Genetic Epidemiology, King's College London, London, $U K$

${ }^{2}$ Clinical Age Research Unit, Department of Clinical Gerontology, King's College Hospital, London, UK

Background: Cognitive impairment is a serious debilitating concern associated with an aging population. Experiments using rodents have linked the gut microbiome and to cognitive function suggesting low microbial diversity is linked to poor cognition function. Here we investigate these findings in a human twin cohort with the aim of identifying novel associations which could be targets to encourage healthy cognition in an ageing population. Materials and Methods: We analyzed 16S rRNA gene sequence data derived from fecal samples obtained from 1,551 individuals in the TwinsUK cohort. Cognitive data was collected using four cognitive tests: Verbal fluency (VF) $(n=1368)$, Deary-Liewald Reaction Time Test (DLRT) $(n=873)$, Mini Mental State Examination (recall) $(n=1374)$ and Paired Associates Learning from the Cambridge Neuropsychological Test Automated Battery (CANTAB PAL) $(n=405)$. Mixed effects models were used to identify associations with alpha diversity, operational taxonomic units (OTUs) and taxa. Models were adjusted for physical frailty, proton pump inhibitor (PPI) and antibiotic use, and diet. Results: DLRT and VF had significant negative associations with alpha diversity of the gut microbiota (FDR adjusted $p \leq$ $.05)$. However, after including physical frailty as a covariate only associations between the DLTR and diversity measures remained. Turicibacter gen significantly associated with CANTAB PAL that also survived correction for frailty. We repeated microbiota associations analyses for the four cognitive phenotypes also including PPI, antibiotic use, and dietary intake as covariates. In these limited subsets we identified several novel associations between DLRT and the class Betaproteobacteria, the genus Lachnospira and the child order Burkholderiales, we observed DLRT to have significant negative associations with all four alpha diversity metrics, reflecting results from the continuous models. In ordinal model for VF we observed novel OTUs assigned to the genus Oscillospira and Clostridiales. Conclusions: We demonstrate a negative association between cognitive performance and gut microbiota diversity and various taxa which remain significant after adjusting for frailty that accounts for physical health. We cannot be sure if these relationships between cognition, frailty and the microbiome are causal or consequential. Nevertheless they represent targets for diagnostic surveillance in cognitive ageing, or for intervention studies to improve vitality.

\section{ACCEPTANCE OF VAGINAL MODE OF DELIVERY IN TRIPLET} PREGNANCIES

Assaad Kesrouani', Elie Choueiry ${ }^{2}$, Inaam Hatoum' ', Bernard Nasr ${ }^{3}$, Habib Atallah', and Ramzi Finan'

${ }^{I}$ Obstetrics and Gynecology Department, St Joseph University, Beirut, Lebanon

${ }^{2}$ Pediatrics Department, St Joseph University, Beirut, Lebanon

${ }^{3}$ Fetal Care Center, Beirut, Lebanon

Introduction: Patients with triplets encounter many problems before and during pregnancy. Patients are often reluctant to undergo 
vaginal delivery in these cases. Materials and Methods: This was a retrospective study at a university hospital from 2008 to 2016 . Patients with a triplets pregnancy above 23 weeks were included, with an obstetrical outcome that was considered acceptable by the obstetrician. We excluded cases with maternal medical problems, history of cesarean or myomectomy, and placenta previa. We considered age, IVF history, preterm labor, maternal wish for the way of delivery, preterm labor, trial of labor and final outcome for delivery. Results: Eleven patients were included in this study. Mean age was 28 years [22-39]. Prior IVF was reported in $7 / 11$ patients $(63 \%)$ and ovarian stimulation by oral or injection in 4/11 (37\%) with no spontaneous cases of triplets. No major obstetrical problem was reported except preterm labor, which occurred in all patients, treated with a mean latency period of 14 days [1-74]. Two patients had cholestasis. In all cases T1 presentation was cephalic. After discussing benefits and risks of vaginal birth, couples were asked to think about it and give their answer when ready. Only 3/11 (27\%) gave their approval. The remaining explained it by 'not wanting the risk' and five said that they took the advice of their family. It is noted that the group who said no were all IVF patients. Among the three patients who accepted vaginal delivery, one patient changed her mind and asked for a cesarean upon admission. The second patient had a spontaneous labor at 35 weeks but finally had a cesarean section at $5 \mathrm{~cm}$ dilation because of an abnormal fetal heart in one of the babies who was small for gestational age. The third patient went into labor at 36 weeks and delivered smoothly with an external version of T2 and T3. Babies had a normal outcome. Conclusions: Vaginal delivery is still feared by pregnant women with triplets. Despite an advanced hospital setting and detailed explanations, most of them chose a cesarean section. IVF history and family advice pushed this option.

\section{ISOLATED ECTOPIA CORDIS IN A TWIN FETUS: A CASE REPORT}

Habib Atallah', Camille Bou Saba ', Linda Daou ${ }^{2}$, Antoine Germanos ${ }^{3}$, and Assaad Kesrouani'

${ }^{I}$ Obstetrics and Gynecology Department, St Joseph University, Beirut, Lebanon ${ }^{2}$ Pediatrics Department, Cardiopediatrics, St Joseph University, Beirut, Lebanon ${ }^{3}$ Saint Joseph Hospital, Dora, Beirut, Lebanon

Introduction: Ectopia cordis is a rare congenital abnormality with a sporadic incidence of 6 per one million live births and may be confused with Cantrell pentad, which includes other associated malformations. Case Report: We report a case of a twin fetus with a complete isolated ectopia cordis in a dichorionic diamniotic twin gestation. There is no consanguinity, family history of abnormalities, IVF or first-trimester medication. Diagnosis of this isolated anomaly was done at 23 weeks in one fetus while the other had normal morphology; The pregnancy was complicated by preterm labor at 28 weeks and cesarean delivery followed at 33 weeks. The affected baby weighed $1,800 \mathrm{~g}$ and had an outcome complicated by episodes of desaturation and deceased at day 8. Parents did not wish in-utero termination of pregnancy or postnatal surgery for reintegration in the thorax and chose palliative care. Videos available. Conclusion: Isolated ectopia cordis can occur in twin pregnancy with a variable outcome.

\section{THORAGOPAGUS TWINS IN A TRIPLET PREGNANCY: A CASE REPORT}

Assaad Kesrouani ', Inaam Hatoum' ', Bernard $\mathrm{Nasr}^{2}$, Habib Atallah', Samer Maalouf', and Ramzi Finan'

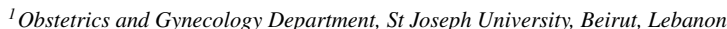
${ }^{2}$ Fetal Care Center, Beirut, Lebanon

Introduction: The incidence of conjoined twins is estimated at 1 in 50,000 births. The timing of ultrasound diagnosis seems to be correlated to the degree of organ sharing and the severity of the case. Case Report: A 38-year-old G1P0 underwent IVF-ICSI for male factor.
Three embryos grade A were transferred at day 3. The first-trimester scan at 12 weeks showed in one sac a normal evolving pregnancy with normal biometry and NT $(1.2 \mathrm{~mm})$. In the other sac, there was a twin thoragopagus pregnancy. One of these fetuses also had an encephalocele with a visible defect at the occipital region. Two hearts were distinctively seen in each side with a complex configuration, the smaller heart being in the fetus with encephalocele, and they were united by at least two vessels. Lower limbs were difficult to assess, and upper limbs were normal. Liver fusion was also suspected at that time. After thorough explanations, the parents chose to terminate that pregnancy. This was done with $\mathrm{KCl}$ intracardiac injection. The other fetus evolved normally. The patient had a vaginal delivery at 38 weeks with a favorable outcome. The thoragopagus baby looked macerated but with recognizable features. Conclusions: Thoragopagus twins is a rare congenital anomaly that can be diagnosed at the time of the first trimester scan to offer the parents an option for pregnancy termination.

\section{COMPARING AMNIOTIC FLUID ECHOGENICITY IN MULTIPLE PREGNANCY WITH IUGR}

Assaad Kesrouani' ', Elie Choueiry², Inaam Hatoum' , Elie Chalhoub' , Bernard $\mathrm{Nasr}^{3}$, Elie Attieh', and Ramzi Finan'

1.Obstetrics and Gynecology Department, St Joseph University, Beirut, Lebanon ${ }^{2}$ Pediatrics Department, St Joseph University, Beirut, Lebanon

${ }^{3}$ Fetal Care Center, Beirut, Lebanon

Introduction: Amniotic fluid echogenicity is rarely evaluated in the medical literature, mainly because of lack of an exact parameter to evaluate. We report a triplet pregnancy where a difference between the different sacs was noticeable for the IUGR fetus. Case Report: A 32-year-old G1 P0 with IVF leading to tri-chorial triamniotic triplets had a regular obstetrical follow-up. First-trimester ultrasound was normal with all babies having normal NT and a basic morphology scan without any particular features. No vaginal bleeding or amniocentesis are reported. Starting at 22 weeks, one of the babies had an impaired growth but was around the 10th percentile. Amniotic fluid index, umbilical and middle cerebral artery Doppler were normal. Follow-up at 32 weeks showed a decreased growth with $\mathrm{T} 2$ about 3 rd percentile and $\mathrm{T} 1$ and $\mathrm{T} 3$ were between 10th and 50th percentile. Amniotic fluid was within normal for all babies and showed no abnormalities on the abdominal scan. An endovaginal scan aimed to measure the cervical length as the patient was complaining of pelvic pain, showed a cervical length of $22 \mathrm{~mm}$; there also was a difference in the amniotic fluid appearance between $\mathrm{T} 1$ and T2, the latter being more echogenic, compared to the other sac. The patient went into labor two days later and was delivered by cesarean section with babies weighing respectively $1,790 \mathrm{~g}, 1,340 \mathrm{~g}$ and 1,490g for T1, T2, and T3. Amniotic fluid in all sacs looked clear without evidence of meconium. Infectious workup was negative. Babies did well in NICU and were in good health upon discharge. This case report was approved and registered by our institution's review board under the number CEHDF-904. Conclusion: Endovaginal scan seems to give better information about the amniotic pattern. Even though no clear correlation exists in the medical literature between amniotic echogenicity and IUGR, this case report raises the question about such an association.

\section{ESTABLISHING A TWIN REGISTER: WONDERFUL RESOURCES FOR PHENOTYPING AND BIOBANKING}

Dorret I. Boomsma

Netherlands Twin Register, Vrije Universiteit, Amsterdam, the Netherlands

Twin cohorts provide unique opportunities for assessing of the role of genetics in the etiology of complex human traits. Because twins and their families are often highly motivated participants, they offer unique opportunities for longitudinal follow-up, leading to rich datasets for studying development and aging, as demonstrated, for 
example, by the Australian, Dutch, British, and Scandinavian twin registers. Twins share environment throughout fetal periods and early years of life, and twin designs harmonize this component of complex traits. The classical twin design compares resemblance in mono- and dizygotic twin pair for uni- or multivariate traits and not only estimates the extent to which genes contribute to variation in single traits, but also the extent to which comorbidity and covariation among traits can be explained by genetics. Because twins are born in all strata of society, twin cohorts tend to be truly population based. Increasingly, registers collect biological samples for DNA isolation, to assess zygosity and take part in genome wide association and epigenetics studies; RNA for transcriptomics studies and multiple tissues for biomarkers. In this symposium, new developments in recruitment and collection, phenotyping, and 'omics' studies will be presented and discussed.

\section{THE NEW MOSCOW NEWBORN TWIN STUDY EXPERIENCE}

Veronika Odintsova' and Sergey Malykh ${ }^{2}$

${ }^{1}$ SPCDM, Moscow Healthcare Department, Moscow, Russia

${ }^{2}$ Psychological Institute of Russian Academy of Education, Moscow, Russia

The purpose of the Moscow Longitudinal Study of Newborn Twins (MLSNT) is to conduct a study aimed at investigating the effect of different genetic and pre- and postnatal environmental factors on child health and mental development. Data on health, cognitive, socio-emotional, biological, and physical characteristics will be collected at five time points: 1 st and 2 nd measures are be conducted during pregnancy, 3rd at birth, and 4th and 5th during the first year after birth. All families are recruited through family planning clinics during pregnancy. The data of MLSNT allows hypotheses to be tested about the influence of genetic, prenatal and postnatal environmental factors on cognitive, emotional and behavioral characteristics of children, as well as to identify specific genetic polymorphisms and describe the epigenetic processes involved in the development of these characteristics. The planning of the MLSNT study has required careful planning: project presentation to the state healthcare structures to receive their support; elaboration of protocols that can permit comparison with international data; provision of legal aspects of the project (approval of the Ethical Committee, informed consent, formalized agreement between researchers about use of data collection); recruiting pregnant women, particular with prejudice toward biosample collection, anxiety about the pregnancy; psychological aid to recruited mothers who have lost one of their children or who had a negative labor experience; maintaining contact with recruited families; correct conservation of biosamples before receiving the grant financing support for expensive data analysis; use of high-throughput human methylome analysis tools. We will discuss the problems of setting up a new longitudinal genetically informative study.

\section{INFANT MORTALITY RATES AMONG ZYGOTIC TWINS AND RISK FACTORS: BIRTH WEIGHT DISCORDANCE AND CAUSE OF DEATHS IN JAPAN, 1995-2008}

Y. Imaizumi
The Center for Twin Research, Osaka University Graduate School of Medicine, Osaka,
Japan

Introduction: Infant mortality rates (IMRs) for MZ and DZ twins decreased by $50 \%$ from 1995 to 2007 in Japan. In the present study, birth weight (BW) discordance (BWD) between co-twins was analyzed as a risk factor of IMRs. Materials and Methods: IMRs were estimated using Japanese vital statistics from 1995 to 2008. Data sources were live births (LBs), fetal deaths (FDs), and infant deaths (Ds). To calculate IMRs for MZ and DZ twins, denominators were total live twin pairs at birth: both LBs at birth and (one LB-one FD)/2. Numerators were twin pairs of both LBs-Ds + [one
LB (survivor)-one LB (D)]/2 + [one LB (D)-one FD]/2. IMRs for $\mathrm{MZ}$ and DZ twins are presented as 1,000 twin pairs of infant deaths divided by LB twin pairs. Intrapair BWD was computed by subtracting the $\mathrm{BW}$ of the smaller twin from that of the larger, dividing the difference by the heavier BW and multiplying by 100 . BWD was categorized into ten groups: less than 5\%, and eight groups from 5\%$9 \%$ to $40 \%-44 \%$, and $\geq 45 \%$. Results: The IMR was significantly higher in MZ than in DZ twins in each BWD category except the second lowest BWD category. The IMR for MZ twins was the lowest at BWD 5-9\% (2.5 per 1000 LB twin pairs) and increased up to the largest $\mathrm{BWD} \geq 45 \%$ (58.9). The IMR of MZ twins was significantly lower for BWD 5-9\% (2.2) than the other BWD categories except less than BWD 5\% (2.5). The IMR for MZ twins had a positive association with increased BWD category. As for DZ twins, the IMR was the lowest at less than BWD 5\% (2.3) and the rate was nearly constant up to BWD 20-24\% (2.4), then increased significantly from BWD $25-29 \%$ (3.8) to the highest BWD category (25.1). As for cause of deaths, twin-to-twin transfusion syndrome (TTTS) was assigned the code P50.3 and birth defects were assigned the code Q00-Q99 in the ICD 10th revision. The BWD was computed for both causes of deaths. As for TTTS, the IMR was the lowest at less than BWD 5\% (0.23) and the rate significantly increased from BWD 10-14\% (0.57) to the highest BWD category (27.43). As for birth defects, BWD was categorized into four groups: less than $10 \%, 10-19 \%, 20-34 \%$, and $\geq 35 \%$. The IMRs for MZ (0.95) and DZ (0.60) twins were the lowest at less than BWD $10 \%$ and significantly increased with BWD in MZ twins, whereas the IMR in DZ twins was significantly increased from $20-34 \%$ (2.24) to $\geq 35 \%$ (13.67). Conclusion: Infant mortality rates among zygotic twins increased with BWD. This trend was a remarkable for TTTS.

\section{POSSIBILITIES FOR BIOBANKING IN TWIN REGISTERS}

\section{P. Eline Slagboom}

Section of Molecular Epidemiology, Leiden University Medical Center, Leiden, the Netherlands

Twin registers collect biological samples to combine molecular and biochemical markers with assessed phenotypes. The quality of the biobank at collection and after storage determines the spectrum of molecular data that can be generated from the biomaterial. To perform such collection procedures in well-standardized fashion allows twin registers to link data analysis across longitudinally acquired samples and to comparable sample collections in cohort and patient based studies. We will discuss what methodology is available and preferred when biobanking blood, saliva or other tissue samples for the analysis of nuclear, mitochondrial and metagenomic DNA, at a quality suitable for whole genome sequencing and epigenetic analyses of the genome. The same will be discussed for biobanking samples for RNA analysis of mRNA and small non coding RNA. State of the art molecular epidemiological studies also include metabolomic or glycomic assays to be combined with genomic and phenotypic variation. Technological developments are aimed at miniaturizing the molecular assays allowing biobanks to make maximum use of the biobank collection for current and future research.

\section{GIRLS VERSUS BOYS: A TWO-YEAR FOLLOW-UP IN A SET} OF QUADRUPLETS

Adrian Craciun ', Amalia Stanescu', Sorina Dumitru', Alexandru Filipescu² , and Mirela Stocklosa ${ }^{\text {I }}$

${ }^{\prime}$ Neonatology Department,' Dr. I. Cantacuzino' Clinical Hospital, Bucharest, Romania ${ }^{2}$ Gynecology and Obstetrics Department, 'Elias' University Emergency Hospital, Bucharest, Romania

Introduction: The incidence of multiple births increased significantly in the late 20th century. These pregnancies are associated with a higher rate of neonatal morbidity and mortality. Case Report: We present the neonatal evolution, the growth and 
neurological development from a two-year follow-up of a set of quadruplets. The data were obtained from medical records. The pregnancy with quadruplets was obtained by assisted reproductive technology. The cesarean section was performed at 29 weeks of gestation. The first and the last newborn were girls with birth weight $1,225 \mathrm{~g}$, respectively $1,100 \mathrm{~g}$ and an Apgar score of 7, respectively 6 . The second and the third of the preterm were boys with birth weight $1430 \mathrm{~g}$, respectively $1460 \mathrm{~g}$ and Apgar score of 6 for both. In the first hour after the birth, the girls developed a mild respiratory distress which disappeared in a few hours. Also, they presented episodes of apnea, treated with caffeine, that persisted only for 2 days. The boys developed more severe respiratory distress and they underwent nasal CPAP for two days. They associated episodes of apnea treated with caffeine and were remitted after 8 days in the case of the first boy, respectively 20 days for the second boy. The quadruplets underwent 20,35, 39 and respectively 21 days of parenteral nutrition. All four required blood transfusion. The third newborn associated ulcero-necrotic enterocolitis stage I. After the neonatal period, the growth and the neurological development were in normal ranges and they didn't achieved any significant disease. Conclusions: This case of quadruplets revealed a better outcome for the girls although they had smaller birth weight. By the corrected age of 18 months they had normal weight, height and neurological skills.

\section{YEARS OF TWIN RESEARCH IN AUSTRALIA}

Nicolas G. Martin

Genetic Epidemiology, QIMR Berghofer Medical Research Institute, Brisbane, Queensland, Australia

'Is there a genetic contribution to scholastic performance?' was the motivating question behind the first Australian twin study, of school examination results from 1967. While the study of 150 twin pairs was fairly large by the standards of the time it soon became apparent that it was far too small to reliably estimate all genetic and environmental sources of variation, specifically the separation of additive genetic (A) and common or shared environmental variance (C), as well as dominance or non-additive genetic variance (D), which is positively confounded with A, and negatively confounded with $\mathrm{C}$. Explorations of power of the classical twin study, first by simulation and later by direct analysis, showed that many thousands of pairs would be needed to separate these sources of variance and this became the justification for founding the Australian Twin Registry in 1978 , which through various means of recruitment rapidly grew to $>30,000$ pairs. This has been a resource for hundreds of twin studies across a wide range of biomedical and social science disciplines. Because of the nationwide spread of recruitment, many of the studies using ATR have been by mailed questionnaire or telephone interview or, more recently, online survey. To enable clinical twin studies in Brisbane, the Queensland Twin Registry was started in 1992 and detailed clinical phenotypes have been obtained longitudinally on $>2,000$ twin pairs and their family members. Anticipating the age of molecular genetics, DNA collection from both ATR and QTR was begun in the early 1990s and this has become a highly valuable and much used resource in the GWAS revolution. Examples will be given.

\section{AN ITALIAN TWIN STUDY ON GENDER DIFFERENCES IN ADULTS' EMPATHY HERITABILITY}

Virgilia Toccaceli' , Corrado Fagnani' , Maurizio Ferri' ', Nancy Eisenberg² , Guido Alessandri ${ }^{3}$, Augusto Vitale', and Maria Antonietta Stazi'

${ }^{I}$ Centre for Behavioural Sciences and Mental Health, Istituto Superiore di Sanità, Rome, Italy

${ }^{2}$ Department of Psychology, Arizona State University, Tempe, USA; 3. Department of Psychology, Sapienza, University of Rome, Rome, Italy

Introduction: Empathy plays a central role in prosocial behavior and human cooperation. Its complexity from both phylogenetic and on- togenetic perspectives is high, as shown by previous evidence from developmental and social psychology, cognitive neuroscience, and behavioral evolution. Very few twin studies have investigated, in adult age, innate and environmental effects in empathic expression, and twin research on gender differences in these effects is almost lacking. Thus, our study aimed (1) to shed light on innate and environmental influences on the expression of empathy - and its cognitive (CE), emotional (EE) and social skills (SS) components in adulthood, and (2) to explore gender differences in these influences. Materials and Methods: Study subjects were about 1,700 twins, aged 18-65 years, already enrolled in the population-based Italian Twin Registry. Empathy was assessed with the Italian version (Preti et al., 2011) of the Empathy Quotient (EQ) (Baron-Cohen $\&$ Wheelwright, 2004). Factor analysis was performed to confirm the three-factor structure of the EQ (i.e., CE, EE, SS). Following the twin method, twin correlations in monozygotic (MZ) and dizygotic (DZ) pairs (males and females) were estimated for the total EQ and subscales scores, and univariate genetic model-fitting was carried out. Results: Women showed significantly higher scores than males for the total EQ and for the CE and EE components, while no gender differences emerged for the SS component. For what concerns heritability, women's empathic expression (i.e. total EQ and $\mathrm{CE}$ and $\mathrm{EE}$ subdimensions) was predominantly driven by genetic factors and individual (non-shared) experiences, while for males, no genetic contribution and important influences of both shared and individual environmental factors emerged; age did not appear to moderate empathy heritability in adulthood. Only for the SS subscale, genetic and environmental proportions of variance were similar between genders. Conclusions: This study suggests possible genderspecific innate and environmental influences on the expression of empathy facets (cognitive and emotional), except for social skills (often not considered a core component of empathy). From a phylogenetic perspective, the hypothesis of a mirroring of the gender differences in empathy levels by the gender differences in heritability patterns seems to come out. These findings might be relevant in the design of educational programs aimed to shape and enhance empathic behavior.

\section{TWIN STUDIES OF OBESITY: AN EPIGENETIC AND MICROBIOME PERSPECTIVE}

\section{Jordana Bell}

Department of Twin Research and Genetic Epidemiology, King's College London, London, UK

Obesity is a global health burden and one of the leading risk factors for developing type 2 diabetes, cardiovascular disease, and related metabolic and inflammatory conditions. Twin studies have formed the basis for characterizing genetic and environmental influences on obesity and its related diseases, and now offer an opportunity to explore biological mechanisms that may mediate these risk factors. Work in twins focusing on intermediate traits that capture variation in biological processes from host-gene regulation to host-microbe interactions has given insights into biological changes observed in obesity. Here, we present findings in epigenomics and human microbiome that identify novel molecular characteristics of obesity in twins. The results help improve our understanding of obesity risk pathways, as well as of the molecular changes that arise as a consequence of adiposity towards its progression to adverse clinical outcomes. 


\section{SLEEP QUALITY AND EMOTIONAL SELF-REGULATION IN}

\section{ADOLESCENCE}

\author{
Emanuela Medda', Corrado Fagnani', Guido Alessandri ${ }^{2}$, Davide Delfino ${ }^{3}$, \\ Antonio Arnofi', Cristiano Violani², Cristina D'ippolito', Sabrina Alviti' , and \\ Maria Antonietta Stazi ${ }^{1}$ \\ ${ }^{I}$ Centre for Behavioural Sciences and Mental Health, Istituto Superiore di Sanità, \\ Rome, Italy \\ ${ }^{2}$ Department of Psychology, Sapienza, University of Rome, Rome, Italy; 3. \\ Associazione di Clinica Cognitiva Lazio, Rome, Italy
}

Introduction: Poor or inadequate sleep during adolescence may have negative effects in terms of brain development, mood disturbances and health risk behaviors. Lukowski and Milojevich (2015) found a significant relation between sleep quality and effortful control (EC) in a sample of university students. The construct of EC reflects the temperamentally-based component of emotion-relevant self-regulation, and captures a set of relatively deliberate control functions needed for voluntary and goal-directed behavior. Children high in EC reveal better social competence and are less likely to develop internalizing and externalizing behavior. This study aimed to investigate the relationship between sleep quality and EC in adolescence and to disentangle the role of genetic and environmental factors in explaining this association. Material and Methods: The study sample included 382 adolescent twins enrolled in the populationbased Italian Twin Registry (120 monozygotic [MZ] pairs, 71 dizygotic [DZ] pairs, aged 14-18 years) and their parents. Adolescents and parents self-responded to the Youth Self-Report/11-18 and Child Behavior Checklist/6-18, respectively. Information from both raters was used to capture the construct of EC and its subdimensions (attentional focusing and inhibitory control). Sleep quality was assessed by the Sleep Disorders Questionnaire (Violani et al., 2004), which enables discrimination between good and bad sleepers. Twin pairs were considered discordant for sleep quality when one twin was a good sleeper and his/her co-twin was a bad sleeper. Emotional regulation measures were compared between MZ and DZ sleep discordant twins (McGue et al., 2010). Results: A statistically significant association, adjusted for age and gender, was detected between EC scores - or related subscales - and sleep quality in twins considered as individuals $(\beta E C 0.33, p<.001$; $\beta$ Attentional $0.29, p<.001 ; \beta$ Inhibitory $0.28, p<. .001)$. When further controlling for shared environmental factors and genetic background in the discordant twin-pair analysis, the association between EC and sleep quality weakened in DZ twins $(p=.05)$ and disappeared in MZ twins $(p=.46)$. Conclusions: Our results show that emotional self-regulation and quality of sleep are closely associated in adolescence; furthermore, they provide evidence against a direct causal link from sleep quality to emotional self-regulation and its components.

\section{TWIN PREGNANCIES AND ANEMIA - CASE REPORT}

\section{Milica Vusurovic, Rajko Nikitovic, and Vesna Njezic}

Neonatology Dept Hospital of Gynecology and Obstetrics, KBC Dr Dragisa Misovic, Dedinje, Belgrade, Serbia

Introduction: Twin pregnancies constitute significant risk to both mother and fetuses. Anemia in twins caused by fetomaternal hemorrhage is rarely described. Massive fetomaternal hemorrhage resulting in profound anemia and shock is associated with high perinatal morbidity and mortality. The incidence of 0.2 per 1,000 pregnancies, but in twin pregnancies it is unknown. Case Report: We report a case of chronic massive FMH in a newborn of a 28 -year-old mother with a first twin pregnancy. Medical visits and tests during pregnancy, including ultrasound scans, were performed. In pregnancy at 35.6 g.a., her obstetrician noted markedly decreased fetal movements of the first twin. Doppler ultrasound detects changes in the pattern of blood flow through the baby's circulation. There was no history of pain, trauma or vaginal bleeding. On arrival at the deliv- ery unit, cardiotocograph (CTG) showed reduced beat to beat variability with variable deceleration for which the mother underwent emergency lower segment cesarean section (LSCS). Outcome was two baby girls. The first baby's weight was $2,550 \mathrm{~g}$, length $47 \mathrm{~cm}$, head circumference $32 \mathrm{~cm}$, and Apgar scores were 5 and 7 at 1 and 5 minutes. The second baby's weight was $2,720 \mathrm{~g}$, length $48 \mathrm{~cm}$, head circumference $33 \mathrm{~cm}$, Apgar scores were 8 and 9 at 1 and 5 minutes and her hematological results were in normal range. The first baby at birth was markedly pale with respiratory depression, responding to bag and mask ventilation and oxygen. The child was tachypneic, audible systolic murmur 3/6. The liver was palpable 2 $\mathrm{cm}$, the spleen was palpable $1 \mathrm{~cm}$. Our initial impression was severe fetal anemia leading to birth asphyxia. The cause of fetal anemia was most likely to be due to fetomaternal hemorrhage. Hematological results-Albumin 18g/L, CBC - Hg 46g/L, Er1,17x10 12/L, $\mathrm{Htc} 0,148 \mathrm{~L} / \mathrm{L}, \mathrm{Wbc} 34,1 \times 10^{9} / \mathrm{L}$, PLT 256x10 $/ \mathrm{L}$, Retics $9,6 \%$. The baby required oxygen, bolus of saline and emergency red blood cell transfusion. The Kleihaure-Betke test (KB test) was done, the fetal hemoglobin level in maternal blood was $6 \%$. Conclusions: Manifestation of FMH depends on the magnitude and the acuity of blood loss. Therefore, it is very important to recognize signs of fetal distress. Diagnosis of FMH can be confirmed by fetal RBC cells in the maternal blood with KBT.

\section{TAPS WITH SIUGR: A NEW ENTITY?}

Manuela Tavares de Sousa, Andreia Fonseca, and Kurt Hecher Department of Obstetrics and Fetal Medicine, University Medical Center Hamburg-Eppendorf (UKE)

Twin anemia polycythemia sequence (TAPS) complicates up to $6 \%$ of monochorionic (mc) twin pregnancies. Diagnosis is based on Doppler Ultrasound findings with an increased MCA-PSV in the donor $(>1.5 \mathrm{MoM})$ and a decreased MCA-PSV in the recipient $(<1.0 \mathrm{MoM})$. Selective intrauterine growth restriction (sIUGR) complicates up to $10 \%$ of MC twin pregnancies. We report three cases (Nos. 1, 2, 3) of MC twin pregnancies with clinically TAPS Stage III that prenatally did not fulfill the Doppler criteria of TAPS in the donor. Furthermore, these cases showed large birth-weight discordances, indicating sIUGR. All placentas showed prenatally clearly different echogenicity and thickness. The recipient twins showed cardiac compromise, such as atrio-ventricular valve regurgitation and cardiac hypertrophy. The MCA-PSV were 0.76 (1), 0.77 (2) and 0.86 (3) MoM, respectively, according to the Doppler definition of TAPS. However, the donor twins had MCA-PSV measurements of 1.16 (1), 1.44 (2) and 1.35 (3) MoM, not fulfilling the criteria of TAPS. All recipients showed polyhydramnios. All cases were delivered by cesarean section at $30+6(1), 29+4$ (2) and $30+5$ (3) weeks of gestation, respectively. Birth-weight discordances were $24.4 \%$ (1), $38.7 \%$ (2) and $22.2 \%$ (3). Postnatally, all were confirmed having TAPS with an intertwin hemoglobin level difference of 10 $\mathrm{g} / \mathrm{dl}$ [1], $8 \mathrm{~g} / \mathrm{dl}$ [2] and $9 \mathrm{~g} / \mathrm{dl}$ [3] and placental injection showed thin arteriovenous anastomoses. To conclude, we believe that these cases of mc twin pregnancies showing TAPS in combination with sIUGR owing to unequal placental sharing present a specific entity of TAPS without severe anemia in the donor, but with cardiac compromise and polycythemia in the recipient. 


\section{OBJECTIVELY MEASURED PHYSICAL ACTIVITY PROFILE} AND COGNITION IN FINNISH ELDERLY TWINS

\author{
Paula Iso-Markku', Katja Waller ${ }^{2}$, Eero Vuoksimaa ${ }^{3,4}$, Henri Vähä-Ypyä ${ }^{5}$, Noora \\ Lindgren $^{6}$, Kauko Heikkilä ${ }^{3}$, Harri Sievänen ${ }^{5}$, Juha Rinne ${ }^{7}$, Jaakko Kaprio ${ }^{3,4}$, and \\ Urho M Kujala ${ }^{2}$ \\ ${ }^{I}$ Department of Clinical Physiology and Nuclear Medicine, HUS Medical Imaging \\ Center, Helsinki University Central Hospital and University of Helsinki, Helsinki \\ ${ }^{2}$ Faculty of Sport and Health Sciences, University of Jyväskylä, Jyväskylä \\ ${ }^{3}$ Department of Public Health, University of Helsinki, Helsinki \\ ${ }^{4}$ Institute for Molecular Medicine Finland, University of Helsinki, Helsinki \\ ${ }^{5}$ The UKK Institute for Health Promotion Research, Tampere \\ ${ }^{6}$ Turku PET Centre, University of Turku, Turku \\ ${ }^{7}$ Clinical Neurology, Turku PET Centre, University of Turku, Turku; Finland
}

Introduction: Physical activity (PA) has been associated with better school performance, better cognition in midlife and reduced incidence of dementia in late life. Few studies addressing PA and cognition have used objectively measured PA instead of questionnaires. We studied objectively measured PA in elderly Finnish twins. Materials and Methods: The study cohort of this cross-sectional study is a part of the older cohort of the Finnish Twin Cohort Study (the twins born in Finland before 1958 and both co-twins were alive in 1967). The twins born in 1940-1944 and who have filled in health questionnaires in 1975, 1981, and 1990 were invited to telephone interview measuring cognitive functioning followed by a week of PA monitoring with a waist-worn accelerometer (Hookie AM20, Traxmeet Ltd, Espoo). Their mean age was 72.9 years with data from 726 persons including 250 full twin pairs). The mean daily metabolic equivalent (MET), daily step count, the total time spent in sitting or lying down (SB), light PA (LPA) and moderate to vigorous PA (MVPA) were examined in relation to cognition in linear regression models. In between-family analyses, twins were compared to others as individuals and in within-family analyses, the intrapair differences in $\mathrm{PA}$ or SB were regressed on intrapair differences in cognition. $R e$ sults: In-between family analyses, LPA was positively and SB was negatively significantly associated with cognition in the final model adjusting for age, sex, education level, living condition, body mass index and for SB also for wearing time (SB: $\beta$-estimate -0.21 , 95\% CI [0.42, -0.003]), LPA: $\beta$-estimate $0.30,95 \%$ CI [0.02, 0.58]). In within-family analyses, no significant association was found between PA and SB with cognition. Conclusions: LPA and SB are significantly associated with cognition among Finnish twins in their seventies when fully adjusted. According to the within-family analyses, the association is likely to be attributable to shared sources of genetic and environmental similarity. Future studies should use genetically informative data such as families or twins or Mendelian randomization studies to uncover in which extent the earlier seen phenotypic associations of PA and cognition are explained by genetic and environmental factors common to both PA and cognition.

\section{A RARE CASE OF AMNIOTIC FLUID DISCORDANCE IN DICHORIONIC TWINS}

Andreia Fonseca, Manuela Tavares De Sousa, and Kurt Hecher

Department of Obstetrics and Fetal Medicine, University Medical Center

Hamburg-Eppendorf (UKE), Hamburg, Germany

Introduction: Twin-to-twin transfusion syndrome (TTTS) is a wellknown complication of monochorionic pregnancies. Extremely rare cases of TTTS have been reported in dizygotic twin pregnancies as well. In cases of amniotic fluid discordance other rare diagnosis should also be considered. Case Report: We report a case of amniotic fluid discrepance in a dichorionic pregnancy in a woman whose previous monochorionic pregnancy was complicated by TTTS. The patient was referred at 23 weeks owing to amniotic fluid discrepance and cervical insufficiency with a suspicion of TTTS. Upon ultrasound examination, oligohydramnios/polyhydramnios sequence was confirmed (deepest vertical pockets were $3 \mathrm{~cm}$ and $12 \mathrm{~cm}$, respectively), as was cervical insufficiency (cervix length $12 \mathrm{~mm}$ ).
However, ultrasound clearly showed dichorionicity. The twins were discordant for fetal sex. The fetus with polyhydramnios had an estimated fetal weight at the 72nd centile and the other twin at the 34th centile. Both twins presented with normal bladder fillings. All Doppler findings were normal. No specific morphologic anomalies were identified. Further placental examination revealed a chorioangioma $(30 \times 28 \mathrm{~mm})$ in the placenta of the larger fetus. At 25 weeks, macrosomia of this fetus was evident (estimated fetal weight above the 97th percentile), associated with an estimated $25 \%$ twin weight discordance. At $26+5$ weeks, following preterm labor with spontaneous rupture of membranes, a cesarean section was performed. Postnatal assessment confirmed the birth weight discordance. The macrosomic neonate developed massive macroglossia and the genetic investigation revealed Beckwith-WiedemannSyndrome (BWS). Histological examination of the placenta confirmed the prenatal diagnosis of chorangioma. Conclusions: BWS is a genetic growth disorder, typically presenting with macrosomia, macroglossia and polyhydramnios. Concomitant chorioangiomas have also been described. This case report shows that sometimes ultrasound findings may mimic TTTS, but dichorionicity and discordant twins' sex make this complication extremely unlikely and other rare diseases should be taken into consideration.

\section{FIRST EXPERIENCE IN SELECTIVE FETAL TERMINATION IN MONOCHORIONIC TWIN PREGNANCIES BY BIPOLAR CORD COAGULATION IN SERBIA}

Vesna Mandic Markovic, Zeljko Mikovic, Dejan Filimonovic, and Nikola Cerovic High-risk pregnancy Department, University Clinic for Gynecology and Obstetrics 'Narodni front', Belgrade, Serbia

Introduction: Selective fetal termination in monochorionic pregnancies is possible only by complete separation of twins' blood flow. Umbilical cord occlusion may be done by fetoscopic cord ligation, interstitial laser coagulation, monopolar coagulation, or ultrasoundguided bipolar cord coagulation (BCC). The aim of this study is to presents the first experience in selective fetal termination in monochorionic pregnancies by ultrasound-guided bipolar cord coagulation in Serbia. Materials and Methods: Since 2014, 12 patients with $\mathrm{MC}$ twin pregnancy were eligible for cord occlusion. All the procedures were done under general anesthesia, antibiotic profylaxis and 48 hours tocolysis. Under the ultrasound guidance a $3.3-\mathrm{mm}$ trocar was introduced into the amniotic cavity and a 3-mm bipolar forceps was passed down the trocar and directed towards the cord insertion of the terminating twin and the cord was grasped with the forceps. Coagulation started at the power of $26 \mathrm{~W}$ for 15 seconds and increased by $5 \mathrm{~W}$ if necessary. The effect was monitored by the appearance of turbulence and steam bubbles and hyperechogenic cord. The procedure was repeated in two other sites for safety. Results: Gestational age at procedure was $20.32 \pm 1.9 \mathrm{wg}(16+4-23+5 \mathrm{wg})$. Six procedures were done in cases of selective fetal growth restriction (sFGR); three in TTTS - two in donor and one in recipient twin; two in case of discordant fetal anomaly and one in a case of TRAP sequence. Before $\mathrm{BCC}$ we performed amnioinfusion in five cases $(41.67 \%)$ - three in TTTS and two in sFGR cases. There were no uterine activity and amniotic fluid leakage, nor the immediate postoperative death of the co-twin. Late death of the co-twin occurred in two cases $(16.67 \%)$ and abortion was induced. Gestational age at delivery was $32.83 \pm 5.37 \mathrm{wg}(23+1-39+1 \mathrm{wg})$. Term delivery occurred in 4 patients $(33.33 \%)$, preterm delivery in $6(50 \%)$ and fetal loss in $2(16.67 \%)$. Procedure-to-delivery interval was $88.08 \pm 36.47$ days (33-133 days). Neonatal period was uneventful in all cases and all children are healthy with normal neurodevelopment. Conclusion: Bipolar cord coagulation proved to be easy to perform and safe treatment for selective feticide in complicated MC twins. 


\section{PERINATAL OUTCOME OF TRIPLET PREGNANCIES:}

\section{SINGLE-CENTER EXPERIENCE}

N. Abián Franco, B. Gastón Moreno, S. García Francés, B. Pérez Munárriz, A. E. Safont Gascón, M. Ruiz García, I. Zabaleta Loinaz, C. Larrañaga Azcárate, and J. Zabaleta Jurío

Complejo Hospitalario de Navarra, Navarra, Spain

Introduction: To analyze perinatal morbidity and mortality in triplet pregnancies at a teaching hospital. Materials and Methods: A case series study was performed based on 45 triplet gestations attending at our hospital from 2003 to 2016. Results: A total of 45 triplet gestations were recorded (incidence $0.07 \%$ ). Up to $83.3 \%$ of pregnancies were trichorionic-triamniotic, $16.7 \%$ were dichorionic-triamniotic. All patients were admitted due to preterm labour, admission gestational age was 29.1 weeks on average, with no statistically significant differences according to chorionicity. We objectified statistically significant differences according to chorionicity in length of hospitalization before delivery (trichorionic 24.39 days; dichorionic 7.6 days), resulting in a global main gestational age at delivery of 32.79 days. The 50th percentile of neonatal weight for 33 weeks was 1,655 grams (based on fetal growth percentiles charts calculated from our sample). Delivery by planned cesarean section occurred in up to $59.3 \%$ of cases, and emergency cesarean section in up to $31.9 \%$, with no differences for chorionicity. Analyzing birth order, we found no differences in cord arterial $\mathrm{pH}$ (7.27 on average), neither in Apgar score during first minute ( 7.47 on average) or at 5 minutes ( 8.67 on average). No differences were found either for length of neonatal hospitalization in terms of birth (mean in first fetuses 25.02 days, 20.03 days in second fetuses and 25.68 days in third fetuses) or chorionicity (22.18 days on average in trichorionic group and 24.44 days in dichorionic). There were no differences for severe morbidity rates according to birth order (6.8\% in first fetuses, $13.6 \%$ in second fetuses, $17.8 \%$ in third fetuses) or chorionicity $(12.4 \%$ in trichorionic and $21.2 \%$ in dichorionic). We observed statistically significant correlation in higher rates of severe morbidity and mortality according to less gestational age at delivery. Total mortality rate was $6.7 \%$. There were also statistically significant differences in terms of mortality $(4.8 \%$ mortality rate in trichorionic, up to $2.9 \%$ within first week; $19 \%$ mortality rate in dichorionic, up to $9.5 \%$ stillbirth) but without differences in accordance with birth order. Conclusions: Triplet growth curves remain stable in 50th percentile until 24th weeks of gestation, with further decrease up to 10 th percentile with no signs of latter flattening. Severe morbidity and mortality rates under 33rd week were relatively low (10-20\% and $4.8 \%$, respectively).

\section{CHANGES IN GENETIC AND ENVIRONMENTAL INFLUENCES ON ARTERIOSCLEROTIC TRAITS: A LONGITUDINAL TWIN STUDY}

Adam Domonkos Tarnoki ', Corrado Fagnani ${ }^{2}$, Emanuela Medda ${ }^{2}$, David Laszlo Tarnoki' , Bence Fejer' ${ }^{1}$, Pal Maurovich-Horvat ${ }^{3}$, Maurizio Ferri ${ }^{2}$, Giacomo Pucci ${ }^{4}$, Pierleone Lucatelli ${ }^{5}$, Claudio Baracchini ${ }^{6}$, and Maria Antonietta Stazi ${ }^{2}$

${ }^{1}$ Semmelweis University, Department of Radiology, Budapest, Hungary

${ }^{2}$ Centre for Behavioural Sciences and Mental Health, Istituto Superiore di Sanità, Rome, Italy

${ }^{3}$ MTA-SE Cardiovascular Imaging Research Group, Heart and Vascular Center, Semmelweis University, Budapest, Hungary

${ }^{4}$ Dipartimento di Medicina, Università di Perugia, Perugia, Italy

${ }_{5}^{5}$ Department of Radiological, Oncological and Anatomo-Pathological Sciences, Sapienza University of Rome, Rome, Italy

${ }^{6}$ Department of Neurosciences, University of Padua School of Medicine, Padua, Italy

Introduction: Arteriosclerosis, the anomalous hardening and thickening of the arterial walls, has become the main factor negatively affecting human survival through cardiovascular diseases such as heart attacks, stroke, and peripheral vascular disease. Limited longitudinal evidence is available about the mechanisms underlying vascular aging. Thus, the main aim of this study was to assess the de- gree of variation of some arteriosclerotic measures over time, and to assess the contribution of genetic and environmental factors to this variation. Material and Methods: Study subjects were 368 twins (107 monozygotic [MZ] and 77 dizygotic [DZ] twin pairs), resident in Italy or in Hungary, who underwent a medical examination in 2009/10 (first wave) and 2014 (second wave). Aortic Augmentation Index (AIxao) as a measure of the arterial wave reflection, aortic pulse wave velocity (PWVao) as a measure of arterial stiffness, heart rate (HR), mean arterial pressure (MAP) and intima media thickness (IMT) were assessed during the first and second visit. As a first description of the longitudinal process, the following correlations were estimated for each trait: within-individual/cross-wave, crosstwin/within-wave and cross-twin/cross-wave correlation. Then, bivariate Cholesky models were fitted to decompose the total variance at each wave and covariance between waves into additive genetic (A), shared environmental (C), and unique environmental (E) components. Results: For each trait, at least moderate longitudinal stability was observed, with correlations between waves ranging from 0.23 (IMT) to 0.60 (AIXao). MZ cross-twin/cross-wave correlations were all significant and, in general, substantially higher than the corresponding DZ correlations, with the latter being significant in only one case (AIXao). A model including additive genetic and unshared environmental influences (i.e., AE) best explained the longitudinal data for all the traits. Under this model, genetic continuity was the main source of longitudinal stability, with genetic correlation estimates between 0.36 (IMT) and 0.70 (AIXao). Overlapping genetic factors explained from 55\% (AIXao) to 88\% (PWVao) of longitudinal covariance of the traits. Conclusions: This study shows that, the genetic contribution to the longitudinal stability is high for PWVao and MAP, while moderate for the other traits. Longitudinal changes develop mainly under the effect of genetic and unshared environmental influences.

\section{BIRTHWEIGHT DISCORDANCE IN TWINS - WHAT IS THE PROGNOSTIC VALUE IN THE ABSENCE OF FETAL GROWTH RESTRICTION?}

Joana Lyra' ', Catarina Melo', Teresa Costa-Castro ${ }^{1,2,3}$, Hernâni Gonçalves ${ }^{4}$, Carla Ramalho ${ }^{1,2,3}$, and Nuno Montenegro ${ }^{1,2,5}$

${ }^{1}$ Gynaecology and Obstetrics Department, São João Hospital, Porto, Portugal

${ }^{2}$ Faculty of Medicine, University of Porto, Portugal

${ }^{3}$ S. Instituto de Investigação e Inovação em Saúde, Porto, Portugal

${ }^{4}$ CINTESIS, Faculty of Medicine, University of Porto, Portugal

${ }^{5}$ Public Health Institute of University of Porto, Portugal

Introduction: Fetal growth restriction (FGR) is often related to birthweight discordance (BWD). However, BWD itself has been identified as an independent risk factor for adverse perinatal outcome. We aimed to investigate the outcome of twin gestations with BWD without FGR. Material and Methods: We conducted a retrospective analysis of all multiple pregnancies who gave birth in our institution from January 2010 until May 2016. Pregnancies complicated with structural anomalies, aneuploidies, triplets and twintwin transfusion syndrome were excluded. FGR was considered for birthweights $<10$ th percentile. Outcomes considered were fetal or neonatal death, 5'Apgar $<7$, admission to neonatal intensive care unit (NICU), neonatal morbidity and gestational age at delivery. The outcome of twin gestations with BWD without FGR (group A), with BWD and FGR in at least one twin (group B) and without BWD or FGR (group C) were evaluated and the groups were compared. Three different BWD cutoffs were used $(\geq 18 \%, 20 \%$ and $25 \%)$ and the analysis was stratified by chorionicity. $\chi^{2}$ tests (or Fisher exact test) were performed for categorical variables and Kruskal-Wallis test for gestational age at delivery; $p<.05$ was considered statistically significant. Results: A total of 379 twin gestations were included, with 293 dichorionic (DC) and 86 monochorionic (MC). In group A with 25\% BWD there were 4 DC and $1 \mathrm{MC}$; with 20\%: $14 \mathrm{DC}$ and $4 \mathrm{MC}$; and with 18\%: $19 \mathrm{DC}$ and $6 \mathrm{MC}$. Comparing all groups together (AvsBvsC) we observed significant differences 
in NICU admission for a BWD $25 \%$ and $20 \%$ in DC twins (BWD $25 \%: \mathrm{A}=75.0 \%, \mathrm{~B}=78.3 \%, \mathrm{C}=40.6 \%, p=.002$; $\mathrm{BWD} 20 \%$ : $\mathrm{A}=61.5 \%, \mathrm{~B}=61.5 \%, \mathrm{C}=39.8 \%, p=.023)$. Neonatal morbidity was different between the 3 groups only for a BWD 25\% (A = $100 \%, \mathrm{~B}=78.3 \%, \mathrm{C}=57.2 \%, p=.041)$. Gestational age at delivery was significantly lower in group A of DC twins for BWD 25\%, $20 \%$ and $18 \%(p=.049 ; p=.001 ; p=.013)$. No differences in Apgar 5' $<7$ were found between the 3 groups for any BWD. We also compared groups A versus B and A versus C for a BWD 25\% and $18 \%$, and we found a lower gestational age at delivery of DC twins in group A (25\%: A vs. B $p=.004$, A vs. C $p=.018 ; 18 \%$ : A vs. $\mathrm{B} p=.001 ; \mathrm{A}$ vs. $\mathrm{C} p=.025)$. For the other outcomes no significant differences were observed in groups A versus B and A versus C. Conclusions: Birthweight discordance without FGR in DC twins is associated with lower gestational age at delivery for all the BWD cutoffs tested. A BWD of $25 \%$ was associated with worst neonatal morbidity in all groups.

\section{TWIN REVERSED ARTERIAL PERFUSION (TRAP)}

\section{SEQUENCE: THREE CASES OVER ONE YEAR}

\author{
Joana Lyra', Teresa Costa-Castro ${ }^{1,2,3}$, Tiago Ferraz ${ }^{1,2,3}$, Carla Ramalho ${ }^{1,2,3}$, and \\ Nuno Montenegro $1,2,4$ \\ ${ }^{I}$ Gynaecology and Obstetrics Department, São João Hospital, Porto, Portugal \\ ${ }^{2}$ Faculty of Medicine, University of Porto, Portugal \\ ${ }^{3}$ i3S, Instituto de Investigação e Inovação em Saúde, Portugal \\ ${ }^{4}$ Public Health Institute of University of Porto, Portugal
}

Introduction: TRAP sequence is a rare condition affecting 1:100 of monochorionic (MC) twins and 1:35,000 of all pregnancies. Early intervention is associated with higher survival rate. Even with spontaneous cessation of flow in TRAP twin, some authors reported high pregnancy loss rates with expectant management. We present 3 cases of TRAP sequence, 2 in MC diamniotic (DA) pregnancies and 1 in MC triamniotic (TA) triplet pregnancy. Case Report: Case 1 - a 38-year-old woman (gravida3/para1/abortion1) diagnosed at $10 w+3 d$ with a MC/DA pregnancy complicated by TRAP sequence. At 14 weeks an intrafetal laser was attempted, but it was not possible for technical reasons; at 15 weeks cord occlusion was not performed because of an amniochorial hematoma and the risk associated to the procedure. At 16 weeks the acardiac twin was confirmed not to have blood flow. Regular ultrasound surveillance was maintained: at 31 weeks a fetus with normal growth (EFW P45.2), normal middle cerebral and umbilical artery Doppler and an acardiac twin maintaining no growth or flow. Case 2 - a 33-yearold woman (gravida3/para0/abortion2) with morbid obesity, type 2 diabetes and chronic hypertension was diagnosed with a MC/DA pregnancy complicated by TRAP sequence at $9 w+5 d$. Ultrasound was performed every week and at $16 \mathrm{w}+5 \mathrm{~d}$ spontaneous cessation of blood flow in the acardiac twin was observed. Close ultrasound surveillance was maintained. At 35 weeks, fetal growth restriction was diagnosed and at 37 weeks the labor was induced (healthy newborn, with 2,035g, Apgar 8/9/10). Case 3 - a 25-year-old woman (gravida1/para0) diagnosed with a MC/TA pregnancy complicated with TRAP sequence at $11 \mathrm{w}+6 \mathrm{~d}$. She was proposed to perform cord occlusion of the acardiac twin with laser ablation of the anastomosis between the other two fetuses or cord occlusion of both the acardiac and pump twin at $18 \mathrm{w}$. Ultrasound was made every week, until 15 th week when the death of all the three fetuses was diagnosed. Conclusions: We present two cases of spontaneous resolution of TRAP sequence before 16 weeks of gestation. The third one is an extremely rare situation of a spontaneous MC/TA pregnancy complicated with TRAP, which result in fetal death, according to the reserved prognosis described in literature.

\section{THE GENETIC MECHANISM BEHIND THE DIETARY FAT} INFLUENCE ON FAT TASTE: A TWIN STUDY

\author{
Dongli Liu' ${ }^{1,2}$, Andrew Costanzo', Nicholas S Archer ${ }^{3}$, Caryl Nowson ${ }^{4}$, Konsta \\ Duesing ${ }^{2}$, and Russell Keast ${ }^{1}$ \\ ${ }^{1}$ School of Exercise and Nutrition Sciences, Deakin University, Burwood, Victoria, \\ Australia \\ ${ }^{2}$ CSIRO Health and Biosecurity, North Ryde, New South Wales, Australia \\ ${ }^{3}$ CSIRO Agriculture and Food, North Ryde, New South Wales,Australia \\ ${ }^{4}$ Institute for Physical Activity and Nutrition, Deakin University, Burwood, Victoria, \\ Australia
}

Introduction: Obesity has become a worldwide epidemic that has been associated with genetic and environmental contributors. Among the environmental factors, fat has been identified as a problem nutrient in the obese. Consumption of a high fat has been associated with attenuated taste sensitivity to fatty acid. The genetic mechanism for the fat taste sensitivity-high fat diet relationship was explained in the animal models by the reduced expression of CD36, a putative fat taste receptor with nanomolar affinity to a range of lipid-based ligands. Materials and Methods: Thirteen twin pairs (3 DZ and $10 \mathrm{MZ}$, aged 20-62, BMI $17-36 \mathrm{~kg} / \mathrm{m} 2$ ) were recruited by Twins Research Australia for the study. A twin from each pair was randomly assigned to either a high-fat ( $>35 \%$ energy from fat) or low-fat $(<20 \%$ energy from fat) diet for 8 weeks. Before and after the trial, fungiform papillae biopsy was conducted and RNA was extracted from these samples. The expression level of the fat taste receptor genes CD36, GPR120, GPR43, GPR84 as well as the delayed rectifying potassium channel gene KV1.2 were analysed and compared between the high-fat and low-fat diet using the quantitative RT-PCR. A linear mixed effects model was applied with the twin pairs as a random effect and zygosity as a fixed effect to assess the pair-wise difference of the gene expression levels between the two diets. The statistical significance was set at $p<.05$. Results: The fat taste receptor genes analysed were not differently expressed between the twin pairs at the baseline. After the 8 weeks, CD36 presented significantly higher expression level in the twins following the low-fat diet compared to the high-fat diet $(p=.032)$. The other analysed genes showed similar trend after the dietary intervention, but with no statistical significant results. Conclusions: Dietary fat modulated CD36 expression suggesting it is the dominant taste receptor associated with the genetic regulation following the dietary fat intervention. Moreover, this provides further evidence that CD36 is a candidate fatty acid taste receptor involved with taste sensitivity to fatty acids.

\section{NEURODEVELOPMENTAL OUTCOME OF MONOCHORIONIC TWINS WITH SELECTIVE INTRAUTERINE GROWTH RESTRICTION USING THE BATTELLE DEVELOPMENTAL INVENTORY-2}

Ruben Quintero', Ramen H. Chmait', ${ }^{1,2}$ Eftichia Kontopoulos', Marian E. Williams ${ }^{3}$, Judy Sloop ${ }^{4}$, Carolina Pena-Ricardo ${ }^{5}$, Lisa M. Korst ${ }^{6}$, and Douglas Vanderbilt ${ }^{\top}$

${ }^{I}$ The USFetus Research Consortium, Miami-Los Angeles, USA

${ }^{2}$ Department of Obstetrics and Gynecology, Division of Maternal-Fetal Medicine, Keck School of Medicine, University of Southern California, Los Angeles, USA

${ }^{3}$ Children's Hospital Los Angeles Center for Excellence in Developmental Disabilities, University of Southern California, Los Angeles, USA

${ }^{4}$ JSloop Consulting, LLC, Boynton Beach, Florida, USA

${ }^{5}$ Kaiser Permanente West Los Angeles, Department of Pediatrics, Los Angeles, USA

${ }^{6}$ Childbirth Research Associates, LLC, Los Angeles, USA

${ }^{7}$ Department of Pediatrics, Division of General Pediatrics, Keck School of Medicine, University of Southern California, Los Angeles, USA

Introduction: To compare the neurodevelopmental outcome of monochorionic-diamniotic twins (MCDA) with type II selective intrauterine growth restriction (SIUGR-II) managed in utero either expectantly or with laser. Materials and Methods: Patients diagnosed with SIUGR-II between 16-26 weeks gestational age (GA) were randomly assigned to expectant management (EM) vs laser therapy (LT). Postnatal neurodevelopmental assessment was conducted 
by trained specialists using the Battelle Developmental Inventory (BDI-2). BDI-2 composite and domain (adaptive, personal-social, communication, motor, and cognitive) scores for the appropriately grown (AGA) and growth-restricted (IUGR) twins were compared by treatment arm. Results: Of 25 SIUGR-II patients, 4 declined randomization and chose expectant management (2) or umbilical cord occlusion (2). One patient had a dual fetal demise before randomization. The remaining 20 patients were randomized to either expectant management (EM) (6) or laser therapy (LT) (14). The mean (SD) $\mathrm{GA}$ at diagnosis was no different between the EM and LT groups (21.5 [2.0] vs. 21.1 [2.8] weeks, $p=.7414$, respectively]. However, GA at delivery was significantly lower in the EM vs LT groups (28.3 [1.8] vs. 33.4 [3.8] weeks, $p=.0039]$. At 6 months, all 20 AGA babies were alive, whereas only $3 / 6(50 \%)$ of the IUGR babies in the EM group and 4/14 (29\%) in the LT group were alive $(p=.6126)$. One family in the EM group and two families in the LT group declined BDI-2 assessment, leaving 5 AGA children in the EM group and 12 AGA children in the LT group available for comparison. The mean $(S D)$ age at BDI-2 assessment was no different between the EM and LT groups (75.6 [14.4] vs. 70.7 [18.2] months, $p=.5618$, respectively). There were no significant differences in composite BDI-2 scores for the EM vs LT AGA children (97.4 [10.4] vs. 98.0 [19.6], $p=.8741$ ), nor in any of the subdomain scores. For the IUGR twins, no statistically significant differences were detected in composite BDI-2 scores between the EM and LT (72.0 [31.1] vs. 92.8 [22.1], $p=.643]$, nor in any of the domain scores. The difference in standardized scores between the AGA and the IUGR children was non-significant for both study arms. Conclusions: Neurodevelopmental outcome appears to be similar between expectantly managed or laser-treated SIUGR-II MCDA twins, possibly due to lack of power, despite the significantly different GA at delivery (33.4 vs. 28.3 weeks, $p=.0039$, laser vs. expectant, respectively).

\section{A TWIN MODEL FOR MAKING CAUSAL INFERENCE: APPLICATIONS TO OBSERVATIONAL EPIGENETIC ASSOCIATIONS}

\author{
Shuai Li' ${ }^{1}$ Ee Ming Wong ${ }^{2,3}$, Lucas Calais Ferreira ', Minh Bui' , Melissa C \\ Southey ${ }^{2,3}$, and John L Hopper \\ ${ }^{I}$ Centre for Epidemiology and Biostatistics, Melbourne School of Population and \\ Global Health, University of Melbourne, Parkville, Victoria, Australia \\ ${ }^{2}$ Genetic Epidemiology Laboratory, Department of Pathology, University of Melbourne, \\ Parkville, Victoria, Australia \\ ${ }^{3}$ Precision Medicine, School of Clinical Sciences at Monash Health, Monash \\ University, Clayton, Victoria, Australia
}

Introduction: Whether or not epigenetic associations with diseases/traits found by observational studies are causal, and in which direction, are important issues with clinical and aetiological implications. We have developed a novel approach, ICE FALCON (Inference on Causation from Examination of Familial Confounding), which uses twin data to make causal inference. Body mass index (BMI) has been suggested to have a causal effect on BMI-related blood DNA methylation levels at the majority of 187 cytosineguanine dinucleotides (CpGs) based on a Mendelian Randomisation (MR) analysis (Wahl et al. Nature 2017). Materials and Methods: For 65 female monozygotic twin pairs, we constructed a methylation score (MS) based on the blood DNA methylation levels in our sample and the effect sizes of CpGs reported by Wahl et al. 2017. We fitted three models using generalised estimating equations in which BMI was predictor and MS was outcome: model 1 regressed a twin's MS against her own BMI, model 2 regressed a twin's MS against her co-twin's BMI, and model 3 regressed a twin's MS against her own BMI and her co-twin's BMI. Bootstrapping was used to make statistical inference on the changes in coefficients across models. In such approach, if a cause is predictor and a consequence is outcome, a decrease in co-twin's coefficient is expected from comparing model 2 to model 3. We then used MS as predictor and BMI as outcome and performed the same analyses. Results: From the analysis in which BMI was predictor, a twin's MS was associated with her own BMI (model $^{1}$, Bself $=0.96,95 \%$ CI $\left.[0.45,1.47]\right)$, and with her co-twin's BMI (model 2; Bco-twin $=0.57,95 \%$ CI: 0.06, 1.08). Conditioning on her own BMI (model 3), Bself remained unchanged ( $p=$ .93 ), and Bco-twin attenuated $(p=.03)$ to be 0.03 (95\% CI [-0.50, $0.55]$ ). From the analysis in which MS was predictor, a twin's BMI was associated with her own MS (model 1; Bself $=0.08,95 \%$ CI $[0.01,0.14]$ ), but not with her co-twin's MS (model 2; Bco-twin = $-0.002,95 \%$ CI $[-0.03,0.03]$ ). Conditioning on her own MS (model 3 ), Bself remained unchanged $(p=.16)$, and Bco-twin increased ( $p$ $=.03)$ to be $0.04(95 \%$ CI [0.001, 0.08]). Conclusions: Our results are consistent with the findings by Wahl et al. (2017) that the BMI has a causal effect on the BMI-associated blood DNA methylation level, but not vice versa. Using this application as an example, we suggest that ICE FALCON could be of value in making causal inference from observational epigenetic associations.

\section{TWIN ANEMIA POLYCYTHEMIA SEQUENCE (TAPS) IN THE ABSENCE OF PATENT PLACENTAL VASCULAR ANASTOMOSES}

Ruben Quintero', Mariam Al-Baloushi ${ }^{2}$, Adrian Charles ${ }^{3}$, Eftichia Kontopoulos', Justin Konje ${ }^{3}$, and Kenyab Najat ${ }^{2}$

${ }^{1}$ USFetus Research Consortium, Miami, Florida, USA

${ }^{2}$ Hamad Medical and Research Center, Doha, Qatar

${ }^{3}$ Sidra Medical and Research Center, Doha, Qatar

Introduction: Twin-Anemia-Polycythemia-Sequence (TAPS) is thought to occur from chronic unbalanced blood exchange between two monochorionic twins (MCTs) through small placental vascular anastomoses. However, this single etiological mechanism has recently come into question. We report the occurrence of TAPS in 2 patients with MCTs in the absence of patent placental vascular anastomoses (PPVAs). Case Reports: Case 1. A 37-year-old, G2, P0 underwent laser therapy for Quintero Stage II twin-twin transfusion syndrome (TTTS) at 22 weeks' gestation. A total of 4 arteriovenous anastomoses were identified and photocoagulated. Follow up ultrasounds showed resolution of the syndrome. The patient was delivered by cesarean section at 31 weeks. The recipient twin weighed $1,674 \mathrm{~g}$, had a hematocrit (hct) of $68.9 \%$ and survived intact. The donor twin weighed $1269 \mathrm{~g}$, had a hct of $22.3 \%$ and died on day 4 from respiratory distress syndrome and Grade III intraventricular hemorrhage. The placenta was examined fresh, weighed $810 \mathrm{~g}$, with $60 \%$ individual placental territory (IPT) for the recipient twin and 40\% IPT for the donor twin. The 4 photocoagulated areas were identified. Air injection studies showed no PPVAs. Case 2. A 30-year, old, G6, P3, underwent laser therapy for Quintero Stage II TTTS at another center at 25 weeks. Details of the surgery were unavailable. Follow-up ultrasound at 28 5/7 weeks showed the recipient twin to be hydropic. Middle cerebral artery peak systolic velocity (MCA-PSV) was 1.9 MOM and 1.2 MOM for recipient and the donor twin, respectively. There was no evidence of persistent or reverse TTTS. The patient was delivered by repeat cesarean section at $29+4$ weeks for worsening status. The recipient twin weighed $1100 \mathrm{~g}$, had a hemoglobin $(\mathrm{Hb})$ of $7 \mathrm{~g} / \mathrm{dL}$, a hct of $21 \%$ and died at 7 hours of life. The donor twin weighed $900 \mathrm{~g}$, had a Hb of $15.9 \mathrm{~g} / \mathrm{dL}$, a hct of $47.5 \%$, and survived intact. The placenta was examined fresh and weighed $666 \mathrm{~g}$. The IPT for each twin was approximately $50 \%$. There were 5-6 visibly photocoagulated areas. An intraplacental hematoma involving $50 \%$ of the placenta was seen, with large intervillous hemorrhage, areas of avascular villi and fetal vessel thrombosis. No PPVAs were documented on air injection studies. Peripheral blood smear of the demised twin showed severe anemia. Conclusions: TAPS may also occur in the absence of patent placental vascular anastomoses. Further research is needed to understand the pathophysiology of TAPS. 


\section{GWAS FOR DZ TWINNING IN A NEW SAMPLE OF} AUSTRALIAN FAMILIES

Nick Martin

QIMR Berghofer Medical Research, Brisbane, Queensland, Australia

Introduction: DZ twinning runs in families and the predisposing genes can be found by genomewide association scan (GWAS). A previous GWAS of about 1,900 mothers of DZ twins (MODZT) from Australia, the Netherlands, and Minnesota found significant hits for SNPs in two genes with other hits not far below the threshold for genomewide significance which a larger sample might reveal to be significant. Methods: A new sample of about 1,900 MODZTs from Australia and New Zealand, from multiplex families including 'affected' sister pairs, was originally collected for linkage analysis but has now been genotyped using the Illumina GSA array (750k SNPs). At the same time a large unselected population sample ( $n$ $=18,000)$ has been collected and typed on the same chip and can be used as controls. Results: Data cleaning is underway and GWAS results will be presented at the conference.

\section{CHARACTERISTICS AND OUTCOME OF TRAP SEQUENCE:}

\section{A THREE CASE SERIES}

\author{
Bernard Nasr ${ }^{1}$, Doris Elia Nasr ${ }^{2}$, and Assaad Kesrouani ${ }^{3}$ \\ ${ }^{1}$ Fetal Care Center, Beirut, Lebanon \\ ${ }^{2}$ Radiology Department, Bellevue Medical Center, Mansourieh, Lebanon \\ ${ }^{3}$ Obstetrics and Gynecology Department, St Joseph University, Beirut, Lebanon
}

Introduction: TRAP (twin reversed arterial perfusion) sequence/acardiac twinning is a rare anomaly that occurs in monozygotic monochorionic twins with an incidence of $1 \%$ and in 1 in 35,000 pregnancies overall. The anomalous twin appears as a heterogeneous mass, simulating a teratoma or intrauterine fetal demise. The normal twin faces increased morbidity and mortality. We report three cases of TRAP sequence with a variable outcome. Case Series: (1) In Case 1, 27-year-old G1P0 at 21 weeks gestation, with moderate hydramnios on the pump fetus. Bipolar cord occlusion of the feeding vessel of the acardiac mass, no color Doppler was seen in the mass after the procedure, normal follow-up. PPROM at 30 weeks' gestation, delivery by $\mathrm{C}$ section of live male baby weighed 1,780g. (2) Case 2: 22-year-old G1P0 at 19 weeks' gestation, severe IUGR less than the first percentile on the pump fetus with an intermittent reverse flow on the single umbilical artery, the acardiac mass was the same volume of the pump twin, the feeding vessel take origin from the cord occlusion. There was a moderate insufficiency with the disappearance of the $\mathrm{E}$ wave on the tricuspid valve. Under ultrasound guidance cord occlusion by bipolar coagulation was done, persistent severe IUGR with fetal demise at 27 weeks' gestation, the pump fetus weighed 500 g. (3) In Case 3, 29-year-old G2P1, a mass was seen beside a normal growing fetus since the eighth week ofgestation; at 12 weeks' gestation there was an abnormal mass with some cardiac activity with a visible feeding vessel. At 16 weeks' gestation, the growing mass showed negative Doppler color with spontaneous occlusion of the feeding vessel. Delivery occurred at 39 weeks gestation, by repeat $\mathrm{C}$ section of a 3,700 g normal baby.

\section{THE CAREER OF A STAFF SCIENTIST - COMBINING A SCIENTIFIC BACKGROUND WITH ADMINISTRATIVE TASKS IN A RESEARCH ENVIRONMENT}

\section{Inge Petersen}

The Danish Twin Regstry, University of Southern Denmark, Denmark

The topic of the presentation is career development from $\mathrm{PhD}$ to a permanent position in science. My career path goes from a $\mathrm{PhD}$ degree in health science, through a research position as a postdoc to an administrative position as a data manager, project coordinator, and data analyst at the Danish Twin Registry. When entering an esteemed research environment, the combination of analytical skills, obtained through work experience in natural science, and a $\mathrm{PhD}$ in health science provides a solid foundation both for when you work on research projects and at the same time participate in the organization of international research collaborations. Throughout my career, I have been involved in many research projects either as a data manager and/or as a scientist; for instance, I have taken part in a multisite international scientific collaboration based on 16 longitudinal surveys of twin registers from five countries (IGEMS). And recently, due to the EU act on personal data becoming effective as of May 2018, I have been using my administrative experience and skills on the legal issues that this act has given rise to, requiring increasingly careful project management in national as well as international collaborative research projects.

\section{FETOSCOPIC LASER PHOTOCOAGULATION IN}

\section{TWIN-TO-TWIN TRANSFUSION SYNDROME}

K. V. Kostyukov, K. A. Gladkova, V. A. Sakalo, O. V. Eremina, A. I. Gus, and N. K. Tetruashvili

National Medical Research Center for Obstetrics, Gynecology and Perinatology, Moscow, Russia

Introduction: Twin-to-twin transfusion syndrome (TTTS) is the most common severe complication of monochorionic (MC) twin pregnancies, with perinatal mortality rate up to $90 \%$, if untreated. This study aimed to review the perinatal and perioperative outcomes of MC twin pregnancies treated for TTTS by fetoscopic laser photocoagulation (FLP) at the Research Center for Obstetrics, Gynecology and Perinatology, Moscow. Methods: A retrospective review of 87 consecutive patients who underwent FLP of placental anastomoses for TTTS from 2011 to 2017 was carried out. FLP was offered to patients who were diagnosed with TTTS (Quintero Stage II-IV) before 26 weeks of gestation. Studied outcomes were perioperative complications and perinatal survival rates. Results: 87 sets of MC twin pregnancies underwent FLP during the study period. Median gestational age at laser photocoagulation was 21.5 (range 17-26) weeks. Overall, perinatal survival rate, double-infant survival rate and survival rate for at least one twin were $72.3 \%, 35.2 \%$, and $30.1 \%$, respectively. Conclusion: FLP is a feasible treatment for TTTS, with minimal maternal complications. Perinatal survival rates of these patients, managed in our Center, were comparable to those of other international centers.

\section{LONG-TERM PHYSICAL ACTIVITY AS A PREDICTOR OF LATE LIFE OBJECTIVELY MEASURED MODERATE-TO-VIGOROUS PHYSICAL ACTIVITY: A 40-YEAR LONGITUDINAL TWIN STUDY}

Katja Waller' , Henri Vähä-Ypyä², Timo Törmäkangas' , Pekka Hautasaari', Noora Lindgren ${ }^{3}$, Paula Iso-Markku ${ }^{4-5}$; Kauko Heikkilä6 ${ }^{6}$, Juha O. Rinne ${ }^{3}$, Jaakko Kaprio ${ }^{6-7}$; Harri Sievänen ${ }^{2}$, and Urho M. Kujala'

${ }^{1}$ Faculty of Sport and Health Sciences, University of Jyväskylä, Jyväskylä

${ }^{2}$ The UKK Institute for Health Promotion Research, Tampere

${ }^{3}$ Turku PET Centre, University of Turku, Turku

${ }^{4}$ Department of Clinical Physiology and Nuclear Medicine, HUS Medical Imaging Center, Helsinki

${ }^{5}$ University Central Hospital and University of Helsinki, Helsinki

${ }^{6}$ Institute for Molecular Medicine Finland, Helsinki

7 Department of Public Health, University of Helsinki, Helsinki

Introduction: Moderate-to-vigorous physical activity (MVPA) in old age is an important indicator of good health and functional capacity enabling independent living. This study aimed to investigate whether physical activity at age 32-48 years predicted objectively measured MVPA at age 73. Materials and Methods: This prospective Finnish twin study comprised 616 individuals (197 complete twin pairs, including 91 pairs known to be monozygotic) who were born in 1940-1944. Participants had responded to all baseline questionnaires in 1975, 1981, and 1990 and participated in 
accelerometer monitoring at follow-up during 2014-2016 at mean age of 73 years. Mean long-term leisure-time physical activity volume (LT-mMET index) was calculated from the baseline questionnaires and mean daily follow-up MVPA was measured with a waistworn triaxial accelerometer (at least 10 hours per day for at least 4 days). Baseline body-mass index (BMI), smoking, health status and social class were used as adjusting variables. Explanatory power of individual-based linear regression analyses was assessed with Rsquared (R2). Pairwise analyses among discordant twin pairs were done using Wilcoxon matched pairs signed rank test. Results: High baseline LT-mMET index predicted higher amounts of MVPA (increase in R2 $6.9 \%$ after age and sex adjustment, $p<.001$ ) at followup. When adding BMI to the regression model the R2 value for whole model was $17.2 \%$, and further adding baseline smoking, social class and health status this increased to $20.3 \%$. In pairwise analyses no differences were seen in the MVPA volumes among low number of twin pairs discordant for baseline LT-mMET $(n=23$ pairs, median follow-up MVPA 28 vs. $33 \mathrm{~min}, p=.6$ ). Monozygotic twin (MZ) pairs were more similar in MVPA compared to dizygotic pairs (DZ) as intraclass correlation was 0.57 (95\% CI [0.44, 0.68]) for $\mathrm{MZ}$ and $0.28(0.13,0.43)$ for $\mathrm{DZ}$ pairs. Mediation analysis confirmed the role of shared genetic factors explaining much of the associations between midlife and later physical activity variables as genetic correlation in best fitting model was $\mathrm{rG}=0.59(0.44,0.79)$. Conclusions: Low leisure-time physical activity at younger age predicted low MVPA at the old age in individual-based analyses. However, based on the pairwise analyses and quantitative trait modeling, genetic factors underlying midlife physical activity seem to be important determinants of later life MVPA.

\section{SIXTH CRANIAL NERVE PALSY AS THE FIRST MANIFESTATION OF PREECLAMPSIA}

Begoña Gastón, Natalia Abián, Beatriz Pérez, Sonia García, Andrea Safont, Irati Zabaleta, Marta Ruiz, Itxaso Pedroarena, and Carlos Larrañaga

Obstetrics and Ginecology, Complejo Hospitalario de Navarra, Pamplona, Navarra, Spain

Introduction: Visual disturbances are fairly common in women with severe preeclampsia or eclampsia and could be the initial symptom on very rare occasions. Among all the possibilities, abducens nerve palsy during pregnancy is an extremely rare condition and only five cases have been reported in the literature. Case Report: A 32-yearold woman with surgical history of adenoidectomy and appendectomy. Tricorial-triamniotic triplet gestation was achieved through assisted reproduction tecniques (artificial insemination). Hospital admission took place at 25 weeks of gestation due to threatened preterm birth, requiring fetal lung maturation and tocolytic treatment with intravenous atosiban, vaginal progesterone and rectal indomethacin. She was finally discharged at $26+4$ weeks of gestation. The patient was admitted again at $28+3$ weeks of gestation due to bilateral sacroiliac pain resistant to oral analgesic treatment. Because of the clinical suspicion of secondary to pregnancy sacroileitis, intravenous analgesics and strengthening exercises were recommended by traumatologists. During this time, uterine dynamic with a new cervical shortening appeared. Therefore, a third cycle of intravenous atosiban was required. After signing informed consent, a maintenance schedule was stablished up to $32+6$ weeks, according to our protocol. Serial obstetric ultrasonography revealed intrauterine growh restriction of the first twin $(\mathrm{EFG}<\mathrm{p} 3)$ with a normal Doppler study. The other two fetuses had an appropriate gestational age weight. Blood pressure remained within the limits of normality throughout the whole pregnancy. At $31+6$ weeks of gestation, the patient referred blurred vision, diplopia and headache. She did not have any other visual alterations, stomach ache, nausea, vomiting or decreased urine output. She had +1 proteinuria and biochemistry indicating normal coagulation, renal and liver function. Fundoscopic evaluation was normal, as was the rest of the exploration. Neuro- logical examination showed a probably idiopathic left sixth cranial nerve palsy. Trans-carotid Doppler and MRI were performed with no abnormal findings. Despite normal blood pressure, the cranial nerve palsy was suspected to be the first symptom of preeclampsia in this patient. She started using an eye patch, and neuroprotection with perfusion of magnesium sulphate was started. Spontaneously active labor started at $32+2$ weeks of gestation, so the patient had an urgent cesarean section under spinal epidural anaesthesia. The first twin was a male weighing 1,397 g with Apgar 9-10 and pH 7.25 and 7.36 (arterial and venous respectively). The second twin was a female weighing 1,470 g, Apgar 9-10 and venous $\mathrm{pH}$ of 7.29. The third twin was female weighing 1,460 g, Apgar 7-8 and $\mathrm{pH} 7.22$ and 7.24; requiring resuscitation through mask ventilation and aspiration of secretions. All of them were admitted to neonatology due to prematurity. During the early postpartum period, the mother's blood pressure reached severity range and diplopia persisted, with no other symptoms suggestive of gestosis. Biochemistry indicated protein of 405 $\mathrm{mg}$ in 24-h urine and albumin-creatinine index of 159. Diagnosis of preeclampsia was established. Subsequently, there was progressive normalization of blood pressure and improvement of diplopia without complete remission. Two months after childbirth, blood pressure had completely normalized and diplopia had almost disappeared. Conclusions: Preeclampsia is a potentially serious pathology and an important cause of maternal and neonatal morbidity and mortality. Although high blood pressure is the most common way of presentation, it is important to think about preeclampsia in the presence of less frequent symptoms, such as the sixth cranial nerve palsy.

\section{SLEEP AND WELLBEING: QUALITY AND DURATION OF SLEEP RELATE DIFFERENTLY WITH HEALTH-RELATED QUALITY OF LIFE}

Juan J. Madrid-Valero ${ }^{1,2}$, Eduvigis Carrillo ${ }^{1,2}$, Juan F. Sánchez-Romera ${ }^{1,2}$, Francisca González-Javier ${ }^{1,2}$, José M. Martínez-Selva ${ }^{1,2}$, and Juan R. Ordoñana ${ }^{1,2}$

${ }^{1}$ University of Murcia, Murcia, Spain

${ }^{2}$ IMIB-ARRIXACA, Murcia, Spain

Introduction: Health-related quality of life (HRQOL) is affected by multiple factors, including sleep problems. Short sleep duration or poor sleep quality is usually associated with higher prevalence of disease, psychopathology and life dissatisfaction (Paunio et al., 2009; Royuela \& Macías, 1997). In addition short sleep duration is associated with a higher risk of mortality (Hublin et al., 2011). A possible explanation, not yet explored, for the relationship between sleep problems and HRQOL could be through shared genes. Hence, our objective was to investigate this relationship in a twin sample of middle-aged adults. Furthermore, since sleep duration and sleep quality present clear and consistent differences between them, we aimed to explore possible differences in their relationship with HRQOL. Materials and Methods: The sample was composed of 1,471 same-sex twins participating in the Murcia Twin Registry (Mean age $=52.5 ; S D=7.2$ ). Sleep quality and duration were assessed through the Pittsburgh Sleep Quality Index and HRQOL through the EQ-5D questionnaire. Two continuous bivariate Cholesky genetic analyses were performed to fit the data. Results: were transformed into a correlated factor solution. Results: The three phenotypes were modestly but significantly heritable: 0.35 (CI 95\% [0.25, 0.44]), 0.24 (CI 95\% [0.15, 0.33]) and 0.31 (CI 95\% $[0.21,0.40])$ for sleep quality, sleep duration and HRQOL respectively. Phenotypic correlations between HRQOL and sleep problems were not high (-0.27 and 0.11 for sleep quality and sleep duration respectively). The genetic correlation between HRQOL and sleep quality was significant $(r \mathrm{G}=-.24 ; 95 \% \mathrm{CI}[-0.44,-0.01])$ and higher than for sleep duration $(r \mathrm{G}=.08 ; 95 \%$ CI $[-0.20,0.34])$. Conclusions: Our result confirms a moderate but significant association between HRQOL and sleep duration and quality. Moreover, there is a genetic association between these phenotypes, but the genetic relationship of HRQOL with sleep quality is stronger than with sleep 
duration. Our data highlight the difference between sleep quality and sleep duration regarding their association with well-being measures. Sleep quality is a broader concept and shows stronger associations with subjective affective states, what can explain the greater genetic correlation with well-being.

\section{RELATIONSHIP BETWEEN SLEEP AND ANTISOCIAL BEHAVIOR}

Juan J. Madrid-Valero ${ }^{1,2}$, Juan R. Ordoñana ${ }^{1,2}$, and S. Alexandra Burt ${ }^{3}$

${ }^{1}$ University of Murcia, Murcia, Spain

${ }^{2}$ IMIB-ARRIXACA, Murcia, Spain

${ }^{3}$ Michigan State University, Michigan, United States

Introduction: There is a close relationship between sleep and behavior problems. Those children that have poor sleep quality or short sleep duration have an increased probability of physical and verbal aggression, hostility and anger (Randler \& Vollmer, 2013). Such findings are often interpreted via environmental explanations, such that poor sleep directly exacerbates or causes symptoms of aggression and behavior problems. Put differently, it is assumed that, should the child get better or more sleep, the aggression or behavior problems would improve. Critically, however, there are other possible explanations for their association that are just as consistent with published phenotypic associations. One key possibility is that poor sleep and aggression are associated via shared genes, such that the genes predicting poor sleep also predict aggression. The current study sought to simultaneously consider these various possibilities. Our specific objectives were twofold. First, we sought to quantify the magnitude of genetic and environmental influences on sleep and on two dimensions of antisocial behavior (aggression and non-aggressive rule-breaking). Our second goal was to evaluate the possibility that sleep may be associated with aggression and rulebreaking for different etiologic reasons. Materials and Methods: The sample was composed of 1,030 twin pairs (426 monozygotic and 604 dizygotic). The sample was $51.3 \%$ male with a mean age of 8.06 years (range $6-11.96 ; S D=1.45$ ). Aggression (AGG), rulebreaking (RB) and sleep were assessed through the Child Behavior Checklist (CBCL) questionnaire. In order to elucidate the origins of the variance within and the covariance among, AGG, RB, and sleep, we fitted a trivariate Cholesky genetic analysis to the data. Results: These were transformed into a correlated factor solution. Results: Those children who slept 'less than most kids' evidenced notably higher mean levels of both AGG (Cohen's $d$ effect size $=.71$ ) and RB (Cohen's $d=.62$ ) as compared to children with adequate sleep. We found a moderate-strong heritability for these three phenotypes (65\% for AGG, $53 \%$ for RB, and $85 \%$ for sleep). Genetic correlation for sleep and AGG $(r \mathrm{G}=.78)$ was higher than that between sleep and $\mathrm{RB}(r \mathrm{G}=.12)$. Conclusion: As expected, there is a close relationship between sleep and aggressive behavior problems, all three phenotypes being highly heritable. Poor sleep shows a greater genetic overlap with aggression than with $\mathrm{RB}$ behavior.

\section{GROWTH IN EARLY CHILDHOOD AND WHEEZING AT THREE YEARS: NATURE OR NURTURE?}

Sonia Brescianini, Sabrina Alviti, Antonio Arnofi, Lorenza Nisticò, and Maria Antonietta Stazi

Center for Behavioural Sciences and Mental Health, Italian Twin Registry, Istituto Superiore di Sanità, Rome, Italy

Introduction: Many studies have reported an association between growth in early childhood and subsequent asthma and/or wheezing. However, it is still unclear how much of the increased risk comes from shared factors (e.g., maternal) and how much from unshared factors (e.g., epigenetics). Our objective was to use twin data to enlighten on the possible mechanisms of the association between growth and respiratory symptoms like wheezing and asthma. $M a-$ terial and Methods: MUBICOS (MUltiple BIrth COhort Study), a longitudinal twin birth cohort within the Italian Twin Registry (ITR), has been used for this purpose. Three-hundred and sixty twin families were enrolled at birth from 2010 to 2014 in eight Italian hospitals. Information on growth and wheezing were assessed by questionnaire (parents were reporting data from the pediatrician records) at $6,12,18$, and 36 months of age. Growth has been computed as $\mathrm{gr} / \mathrm{kg} / \mathrm{d}$ using the exponential formula and for wheezing, "wheezing ever' at 36 months was used. Other confounding variables used in this analysis were: parental asthma and smoking, maternal age and education, and number of siblings in the household. Regression models for twin studies as described in Carlin et al. (2005) were used. This approach implies the estimation of different models. The first was performed using twins as they were unrelated individuals (adjusting the $S E$ estimates) while the subsequent models estimate the within- and between-pair beta coefficients in order to test whether the association estimated in the first step is confounded or not by maternal/familial factors. Results: Results are available on 154 pairs. About 34\% of the twins had experienced at least one episode of wheezing by 3 years of age while the average growth was about $4 \mathrm{~g} / \mathrm{kg} / \mathrm{d}$. The odds-ratio $(O R)$ of the association between growth and wheezing ever in the whole sample of twins was 1.53 (95\%CI [1.1, 2.1]). The within-pair $O R$ was $1.44(95 \%$ CI $[0.3,6.6])$ while the between-pair $O R$ was $2.15(95 \%$ CI $[1.1,4.4])$. These results did not change when adjusted for the confounding factors reported above. Conclusions: The association seems to be due mostly to maternal/familial factors. These have yet to be explored but could be the same acting in utero in slowing down growth. In fact, infants with low birth weight have a faster growth in early childhood. Further analyses on a bigger sample will help to clarify these patterns to identify ways for public health itnervention.

\section{BIAMNIOTIC BICORIAL TWIN PREGNANCY: CASUAL FINDING OF VASA PREVIA IN FIRST TWIN}

Begoña Gastón, Natalia Abián, Marta Ruiz, Julen Barrenetxea, Irati Zabaleta, Marta Bazán; Sonia García; Maitane Urtasun, and Carlos Larrañaga

Obstetrics and Ginecology, Complejo Hospitalario de Navarra, Pamplona, Navarra, Spain

Introduction: Vasa previa is a condition in which some blood vessels flow through the amniotic membrane that lines the internal cervical orifice, so that they are not protected by Wharton gelatina within the umbilical cord. This increases the risk of fetal hemorrhage following amniotomy. Approximately, its incidence is of 1 in 2,500-5,000 deliveries. The fetal mortality rate may reach $60 \%$ if vasa previa has not been diagnosed before delivery. Once the diagnosis has been obtained by ultrasound, elective cesarean section will be programmed to avoid spontaneous rupture of membranes and a dangerous fetal bleeding. Material and Methods: A case of vasa previa is presented. Results: A 35-year-old patient with a history of idiopathic thrombocytopenic purpura and carrier of a total hip prosthesis due to avascular necrosis of the right hip, secondary to chronic corticosteroid treatment. Double gestation bicorial biamniotic was obtained from assisted reproduction techniques because of primary sterility from the age of 31. First trimester screening shows low risk for trisomy 21. No incidences were reported during first and second trimester of pregnancy. A transvaginal ultrasound was performed at 31 weeks of gestation to assess cervical length. Just above the internal cervical orifice, a structure that looked like umbilical cord was seen; it did not move with fetal movements and presented blood flow with Doppler. Upon diagnostic suspicion of vasa previa of the first twin, serial obstetric ultrasound were performed, confirming the diagnosis. The patient was admitted at $35+2$ weeks. Two doses of intramuscular betamethasone $12 \mathrm{mg}$ were given for fetal lung maturation and cross-test was obtained in case blood transfusion was required. Elective caesarean section was performed with spinal anesthesia. 
After a low transverse segmental hysterotomy, the placental border was dissected until a space of amniotic membrane without vasa previa was reached in order to perform the amniotomy. The first twin was a female weighing 2,300 g, Apgar 9-10 and $\mathrm{pH} 7.36 \mathrm{y}$ 7.40 (arterial and venous respectively). The second twin was a female weighing 2,090 gr, Apgar 9-10 and pH 7.29 and 7.34. They did not need resuscitation or admission to neonatology. Conclusions: Vasa previa is an ultrasound finding that implies the need for strict pregnancy control and elective ending by cesarean section in order to avoid the fetal morbidity and mortality that can be derived from it.

\section{THE HIGHER CONCORDANCE RATE OF BILIRUBIN LEVELS OF MONOZYGOTIC TWINS COMPARED TO DIZYGOTIC TWINS}

You Kyoung Uhm, Seung Mi Lee, Chan-Wook Park, Joong Shin Park, and Jong Kwan Jun

Department of Obstetrics and Gynecology, Seoul National University Hospital, Seoul, Republic of Korea

Introduction: Bilirubin levels are controlled by several factors, including the activity of enzymes which is determined by genetic components. Monozygotic twins (MZT) are a good candidate to differentiate genetic components from environmental factors. We hypothesized that the difference in the bilirubin level is smaller in MZT than in dizygotic twins (DZT) because MZT twins have the same genetic composition while DZT twins share only half. Materials and Methods: This retrospective cohort study was conducted on women with a twin pregnancy who delivered live-born neonates (gestational age at delivery, $\geq 37+0$ weeks) at Seoul National University Hospital between January 2004 and December 2013. We excluded neonates who were transferred to neonatal intensive care unit. Twin zygosity was determined according to the gender of the neonates(different gender $=$ dizygote, $n=324$ ), chorionicity (monochorionic twin $=$ monozygote, $n=120$ ), and DNA analysis (Powerplex 16, Promega) results of the umbilical cord blood at birth $(n=225)$. Transcutaneous (TC) bilirubin concentrations were measured by using a TC bilirubinometer (Minolta Jaundice Meter JM-103). The concordance of bilirubin levels was defined as less than $2 \mathrm{mg} \%$ difference in the highest levels between first and second twins staying at nursery. We used the Chi-squared test and Student's $t$ test, as appropriate. A $p$ value $<.05$ was considered to reflect statistical significance. $R e$ sults: The study population consisted of 162 MZT pairs and 507 DZT pairs. There was no difference in the gestational age at birth according to zygosity ( 37.7 vs. 37.9 weeks, $p=.303$ ). The discordance rate of difference in TC bilirubin was $20.4 \%$ (33/162) versus $28.6 \%(145 / 507)$, with statistical significance $(p=.039)$. Conclusions: MZT twins have a more similar pattern of bilirubin levels than DZT twins. Bilirubin levels may be affected by genetic control.

\section{IMPROVED PERFORMANCE OF VAGINAL DELIVERY IN TWIN PREGNANCY; 15 YEARS' EXPERIENCE IN SEOUL NATIONAL UNIVERSITY HOSPITAL}

Min Kyoung Kim, Seung Mi Lee, Chan-Wook Park, Joong Shin Park, and Jong Kwan Jun

Department of Obstetrics and Gynecology, Seoul National University Hospital, Seoul, Republic of Korea

Introduction: Vaginal delivery is recommended in twin pregnancy that has 1st vertex presentation and no other indication for cesarean section. However, young obstetricians and even some experienced obstetricians are reluctant to try vaginal delivery in twin pregnancy because failed vaginal delivery including combined delivery is not infrequent. Recently, the number of twin deliveries has increased year after year at our center and we showed our experience of trial of labor in twin pregnancy. Materials and Methods: We reviewed 3,026 pairs of twin pregnancies who delivered at Seoul National University Hospital from 2001 through 2015, divided into three groups by five years [2001 2005, Period 1 (P1), 2006 2010, Period 2 (P2), 2011 2015, Period 3 (P3)]. Out of them, we enrolled women with twin pregnancies who underwent trial of labor and whose gestational age at delivery was over 28 weeks(127 in P1, 469 in P2, and 947 in P3). Cases of at least one fetal death were excluded. We compared successful outcome (vaginal-vaginal delivery) and failed outcome (cesarean-cesarean and vaginal-cesarean delivery) among three groups. Statistical analysis was performed using SPSS 21.0 with $\chi^{2}$ test. Results: The number and proportion of twin deliveries was 332 and 6.2\%(332/5317), 906 and 15.1\%(906/6013), and 1788 and $22.8 \%(1788 / 7835)$ in $\mathrm{P} 1, \mathrm{P} 2$, and $\mathrm{P} 3$, respectively. As the years go by, the rate of successful trial of labor increased as follows: $72.4 \%$ (92/127) in P1, 82.9\% (411/496) in P2, and $86.3 \%$ (817/947) in P3, $p<.001$. Decreased rate of cesarean-cesarean delivery and vaginal-cesarean delivery was also found. The cesareancesarean delivery rate decreased as follows: $22.4 \%(31 / 127)$ in P1, $14.7 \%(73 / 496)$ in $\mathrm{P} 2$, and $13.2 \%(125 / 947)$ in $\mathrm{P} 3, p=.002$. The vaginal-cesarean delivery rate decreased as follows: $3.2 \%(4 / 127)$ in $\mathrm{P} 1,2.4 \%(12 / 496)$ in $\mathrm{P} 2$, and $0.5 \%(5 / 947)$ in $\mathrm{P} 3, p=.002$. Conclusions: Likewise other medical fields, more experience of vaginal delivery in twin pregnancy may increase successful outcome. Vaginal delivery of twin pregnancy also needs somewhat long learning curve.

\section{TRIPLET PREGNANCIES REVIEW: AN 11-YEAR EXPERIENCE OF A TERTIARY CARE CENTER IN MADRID, SPAIN}

Sergio Caballero Sanz', Javier Ignacio llabaca Salas ${ }^{2}$, Juan Antonio León Luis', Luana Riolobos Sierra ${ }^{3}$, Carlos Alonso Mayo' , Francisco José Amor Valera', and Santiago García-Tizón Larroca'

${ }^{1}$ Hospital General Universitario Gregorio Marañón, Madrid, Spain

${ }^{2}$ Hospital de Carabineros de Chile, Santiago, Chile

${ }^{3}$ Hospital Clínico San Carlos, Madrid, Spain

Introduction: Multiple pregnancies have increased lately by both the practice of assisted reproductive technology and the trend of advanced maternal age at spontaneous conceptions. Triplet pregnancies remain a challenge, mainly because of preterm birth and low birth weight; but they are also associated with poorer maternal outcomes and increased obstetric complications. Our objective was to describe all triplet pregnancies managed at the Hospital General Universitario Gregorio Marañón over an 11-year period, evaluate neonatal and maternal outcomes, and compare differences in obstetrical results between groups based on maternal and pregnancy characteristics. Material and Methods: We conducted a retrospective study with data collected from medical records of 67 triplet pregnancies that reached $\geq 22$ weeks of gestation (w) between January 2006 and July 2017. We performed a descriptive analysis for maternal and gestational characteristics and perinatal outcomes. A comparative analysis between groups was carried out for preeclampsia, intrauterine growth retardation (IUGR) and preterm delivery below 30 $\mathrm{w}$ taking into account all the variables collected. Results: The mean maternal age at birth was 33.4 years. IVF method of conception was the most common (50\%) and $76.4 \%$ were nulliparous. All patients had a cesarean delivery. The mean gestational age at birth was $32.2 \mathrm{w}$ and mean birth weight $1,622 \mathrm{~g}$. IUGR was the most frequent adverse outcome (50\%), followed by preterm delivery $<30 \mathrm{w}(18.2 \%)$, Apgar at 5 minutes $\leq 7$ of any newborn $(13.2 \%)$ and gestational diabetes $(7.35 \%)$. There were no statistical differences between groups comparing pregnancies complicated either by preeclampsia or by IUGR when analyzing the rest of the variables collected. We did find statistical differences between pregnancies complicated with preterm delivery $<30 \mathrm{w}$ and the rest in terms of birthweight $(1,006 \mathrm{~g}$ versus $1763 \mathrm{~g}, p<.01)$ and perinatal mortality ( $25 \%$ vs. $0 \%, p<.01)$. We also found statistical differences between birthweights when considering the order of birth, being the first newborn mean birthweight 
$1,732 \mathrm{~g}$ and the third $1,533 \mathrm{~g}(p=.014)$. Conclusions: Triplets have a greater risk of prematurity and neonatal morbidities such as IUGR or Apgar at 5 minutes $\leq 7$ of any newborn. Although birthweights between the first and the third newborn were significantly different, more studies are needed to determine whether that discrepancy is clinically relevant.

\section{RELATIONSHIP BETWEEN DNA DAMAGE RESPONSE AND METABOLIC PROFILE IN OVERWEIGHT/OBESE INDIVIDUALS}

Lorenza Nisticò' , Paola Fortini ${ }^{2}$, Francesca Marcon ${ }^{2}$, Valeria Guglielmi ${ }^{3}$, Valeria Simonelli ${ }^{2}$, Serena Laterza ${ }^{3}$, Egidio lorio ${ }^{4}$, Michele Signore ${ }^{4}$, Cristina D'ippolito', Miriam Salemi' ${ }^{1}$, Sonia Brescianini ${ }^{1}$, Carla Ferreri ${ }^{5}$, Paolo Sbraccia $^{3}$, Maria

Antonietta Stazi ${ }^{\prime}$, and Eugenia Dogliotti ${ }^{2}$

1 Centre for Behavioural Sciences and Mental Health, Italian Twin Registry, Istituto Superiore di Sanità, Rome, Italy

2 Department of Environment and Health, Istituto Superiore di Sanità, Rome, Italy 3 Centre for the Cure of Obesity, Department of Systems Medicine, Università di Roma Tor Vergata, Rome, Italy

4 Core facilities, Istituto Superiore di Sanità, Rome, Italy

5 Institute for organic synthesis and photoreactivity, Consiglio Nazionale Ricerche, Bologna, Italy

Obesity is a serious concern because of its high worldwide prevalence and causal relationship with serious medical illnesses, including type 2 diabetes, cardiovascular disease and cancer. Obesityassociated low-grade chronic inflammation is a main risk factor for cancer development. Recent studies suggest that an excessive caloric intake could lead to the production of metabolic byproducts, such as reactive oxygen species or lipid peroxides, which may lead to accumulation of unrepairable DNA damage and chronic DNA damage response (DDR). DDR activation leads to the senescence associated secretory phenotype, which if persistent, may promote age-related diseases. We aim to verify whether the DDR is differently activated in obese and lean subjects and it is correlated to markers of inflammation and obesity, and to study the effect of weight loss obtained either by diet or by bariatric surgery. We have planned an intervention study in two subject groups: (1) pairs of monozygotic (MZ) twins discordant for body mass index (BMI) before and after the heavier twin has undergone diet-induced weight-loss and (2) massively obese patients before and after bariatric surgery. So far, we have enrolled $12 \mathrm{MZ}$ pairs (mean BMI difference $=6.2 \mathrm{~kg} / \mathrm{m} 2$ ) and 9 surgically treated obese patients (mean BMI before surgery $=43.6 \mathrm{~kg} / \mathrm{m} 2$ ). We collect health and lifestyle info, anthropometric measures, hematological parameters and biological materials (blood cells, serum, plasma, urine, feces, buccal wash, visceral and subcutaneous adipose tissue (in patients only). We are currently carrying out lipid profiles in erythrocytes membranes and NMR-metabolic profiles of serum and fat tissue, as well as, phosphoproteomics analysis of DDR markers in isolated peripheral blood mononuclear cells. Preliminary data show that the overweight/obese twin have distinctive profiles of fatty acids methyl esters, accumulation of lactate, VLDL and LDL lipoproteins compared to the leaner co-twin. Final data in BMI-discordant MZ twins will detect those differences that are due to the effect of individual environmental exposures and, more importantly, will suggest if weight loss and DDR are causally related.

\section{EFFECTS OF RECENT STRESSFUL LIFE-EVENTS ON PRONENESS TO PSYCHOSIS IN AN ITALIAN TWIN SAMPLE}

Maria Nobile', Corrado Fagnani ${ }^{2, *}$, Marco Garzitto $^{3, *}$, Carolina Bonivento $^{4}$, Sara Piccin $^{3}$, Maddalena Mauri ', Eleonora Maggioni ${ }^{5-6}$, Miriam Salemi ${ }^{2}$, Antonio Arnofi $^{2}$, Virgilia Toccaceli ${ }^{2}$, Monica Bellina ${ }^{1}$, Massimo Molteni ${ }^{1}$, and Maria Antonietta Stazi ${ }^{2}$

${ }^{1}$ Scientific Institute IRCCS Eugenio Medea, Bosisio Parini, Lecco, Italy

${ }^{2}$ Centre for Behavioural Sciences and Mental Health, Istituto Superiore di Sanità, Rome, Italy

${ }^{3}$ Scientific Institute IRCCS Eugenio Medea, San Vito al Tagliamento, Pordenone, Italy ${ }^{4}$ Department of Medical Area (DAME), University of Udine, Udine, Italy

${ }^{5}$ Department of Basic Medical Science, Neuroscience and Sense Organs, University of Bari Aldo Moro, Bari, Italy

${ }^{6}$ Department of Neurosciences and Mental Health, Psychiatric Clinic, Fondazione IRCCS Ca' Granda, Ospedale Maggiore Policlinico, University of Milan, Milan, Italy

Introduction: Proneness to (non-)affective psychoses was widely investigated through self-report measures. Positive schizotypy showed moderate heritability in twin and family studies, but it is unclear how life events modulate this trait. In fact, personality risk could be both a marker of vulnerability and a reaction to distress, which is strongly associated to disorder onset. Materials and Methods: Among 294 twins enrolled in the population-based Italian Twin Registry, 139 complete pairs (54\% females) aged 8-32 years (mean: 18 \pm 6.7 ) were assessed with scales for positive schizotypy (MIS, Magical Ideation Scale, Eckblad \& Chapman 1983; PAS, Perceptual Aberration Scale, Chapman et al., 1978) and for proneness to affective disorders (HPS, Hypomanic Personality Scale; Eckblad \& Chapman 1986), scoring also subscales of HPS: SV, Social Vitality; MV, Mood Volatility; Ex, Excitement (Schalet et al., 2011). Lifetime stressful events (LEs) were collected with an ad-hoc inventory. Quality of parents' relationship was assessed (RQI, Relationship Quality Index; Norton 1983). Univariate twin models for quantitative traits were fitted on proneness scales in assessed monozygotic ( $N$ 104) and same-sex dizygotic ( $N$ 92) twins. Effects of introducing LEs (last year and lifetime) and RQI in selected models were evaluated. Results: No sex differences were observed for proneness scales. Age negatively correlated with HPS scales ( $r$ ranging from -0.34 to $0.22)$. Lifetime LEs were negatively correlated with SV (-0.17) and HPS (-0.15), while recent LEs correlated with MIS $(+0.18)$ and PAS $(+0.15)$. RQI was negatively correlated with MV (-0.18), HPS ($0.17)$, and MIS (-0.15). An AE model best explained MIS and SV data, with heritability estimates of $42 \%$ and $61 \%$ respectively. Under a CE model, shared environment explained $53 \%, 47 \%$ and $27 \%$ of variance in Ex, HPS and MV scores respectively. Unique environment only affected PAS. Sex showed significant effects in MIS and PAS final models, age in all HPSs ones. Introducing recent LEs had a significant effect on MIS and PAS models; in particular, in MIS model, last-year LEs modified variance explained by A (-8\%) and by $\mathrm{E}(+10 \%)$. Lifetime LEs and RQI did not affect fitted models. Conclusions: Recent stressful events resulted to increase positive schizotypy, while life-time ones reduced extraversion. Parental relationship could protect against neuroticism and positive schizotypy. Moderate heritability resulted for positive schizotypy and extraversion.

\section{INTRA-PAIR BIRTH WEIGHT DIFFERENCES AND BEHAVIOR PROBLEMS IN PRESCHOOL TWINS}

Elena C. Tore ${ }^{1,2}$, Evangelia E. Antoniou' ${ }^{\prime}$ Keith Reed ${ }^{3}$, Taunton R. Southwood ${ }^{4}$, Luc Smits' ${ }^{1}$, Joseph P. Mccleery ${ }^{5}$, and Maurice P. Zeegers'

${ }^{1}$ Maastricht University, Maastricht, the Netherlands

${ }^{2}$ University of Birmingham, Birmingham, United Kingdom

${ }^{3}$ The Twins and Multiple Births Association (TAMBA), United Kingdom

${ }^{4}$ Institute of Child Health, University of Birmingham, Birmingham, United Kingdom

${ }^{5}$ Children's Hospital of Philadelphia, Philadelphia, PA, United States of America.

Introduction: Internalizing and externalizing problem behaviors are of increasing public health concern because of the numerous consequences for the individual and the whole society. Twin 
studies have revealed substantial genetic and environmental influences on children's behavior, while examining birth weight difference could be helpful to identify the specific contribution of various non-shared prenatal environmental factors on later psychological development. Materials and Methods: As part of the Twins and Multiple Births Association (TAMBA) Heritability Study, we recruited mothers of twins aged 18 months to 5 years registered with TAMBA, a volunteer-based association in the United Kingdom. Nine-hundred and sixty twins (480 pairs) were included in this analysis. Twins' mothers answered questions relative to their pregnancy and their twins' characteristics, and completed the Child Behavior Checklist (CBCL) 1.5-5. This widely used tool gives information regarding internalizing and externalizing problems, identifying the more challenging areas for each child. The association between absolute birth weight difference and each CBCL scale's score difference was analysed by means of a multiple linear regression. Expected mean CBCL score differences were calculated. Results: In monozygotic and dizygotic twins analyzed together, statistically and clinically significant associations were found between intrapair birth weight difference and difference in total score and sleep problems scores. These results were repeated in monozygotic twins analysed alone. In addition, the associations of birth weight difference with difference in internalizing problem, emotional reactiveness and somatic complaints were statistically and clinically significant in monozygotic twins. No significant results were observed in dizygotic twins when analyzed as a separate group. Conclusions: The results of the present study show that, with increasing absolute birth weight difference, the intrapair difference in total problems, internalizing behaviors and sleep problems increases, with smaller twins being at major risk of behavior problems. Moreover, results in monozygotic twins suggest a causal association between birth weight and behavior development, as genetic and common environmental influences are automatically controlled for in this type of analysis.

\section{THE USP TWIN PANEL: CURRENT STATUS AND PROSPECTS FOR BRAZILIAN TWIN STUDIES IN PSYCHOLOGICAL RESEARCH}

Marco Antonio Corrêa Varella', Jonas Arantes ${ }^{2}$, Viviana Giampaoli ${ }^{3}$, Eloisa De Souza Fernandes' ', Tiziana G. Acquaviva' ', Tania Kiehl Lucci' ', Sylvia Corte ${ }^{4}$, Noelia Gomes ${ }^{4}$, Nancy L. Segal ${ }^{5}$, Fernando Leite Ribeiro', and Emma Otta'

${ }^{I}$ Department of Experimental Psychology, Institute of Psychology, Universidade de São Paulo, São Paulo, Brazil

${ }^{2}$ Institute of Biosciences, Universidade de São Paulo, São Paulo, Brazil ${ }^{3}$ Statistics Department, Institute of Mathematics and Statistics, Universidade de São Paulo, São Paulo, Brazil

${ }^{4}$ Faculty of Sciences, Universidad de La Republica, Montevideo, Uruguay

${ }^{5}$ Department of Psychology, California State University, Fullerton, USA

The USP Twin Panel, based at the Institute of Psychology of the University of Sao Paulo, was created in April 2017, by a faculty team under the leadership of Professor Emma Otta. The USP Twin Panel is registered at the USP Innovation Agency and the registration process is underway at the Brazilian National Institute of Industrial Property. The panel brings together twins interested in participating in research on psychological processes and in services provided by the University. Our research network brings together faculty and students from universities such as Federal University of Para, University of Brasilia, Catholic University of Salvador, La Universidad de la República (Uruguay) and California State University, Fullerton (USA). In Sao Paulo the Twin Research Project is part of the Center for Applied Research on Well-Being and Human Behavior, the largest scientific nucleus of the country focused on well-being research. There is a notable lack of Brazilian psychological research on twins. Now we have enrolled a total of 935 individuals: 311 parents and 624 twins included multiples. Registration on our Panel can be made in Portuguese or in Spanish, and we have a Facebook page (Painel USP de Gêmeos) with more than 1,300 followers. We have organized two twin meetings to increase personal interaction among twins and researchers. In addition to expanding the registrations at the Panel, our group published a study on a massive demographic database on live birth in São Paulo city (2003-2014), in order fill the information gap regarding twinning rates in our country. Out of a total of 2,056,016 deliveries, the overall average twin rate was $11.96 \%(7.15 \% \mathrm{DZ}$ and $4.42 \% \mathrm{MZ})$, and higher order multiple births was $0.36 \%$. The rise in the twin rate was about $40 \%$ over the period and the best predictor was increased mother's age. Out of an actualized database (2003-2015), we have now analyzed the twinning rates of São Paulo as a function of the district of mother's residence and the type of hospital. We found that there is heterogeneity within the city, with relatively more twins born in private hospitals than in public ones and the higher the average income of mother's residence district. Brazil is a huge and populous country, culturally, ethnically and economically diverse with much to offer for psychological twin research. We expect that the USP Twin Panel will encourage high quality twin studies on psychological processes and behavior in Brazil and South America. Grants: \#2014/50282-5, and 2017/10501-8 of São Paulo Research Foundation (FAPESP) and Natura Cosméticos S.A. 'Despite the fact the USP Twin Panel is registered at the USP Innovation Agency and the registration process is underway at the Brazilian National Institute of Industrial Property, it does not influence our work, so all authors declare no conflict of interest. Our grants \#2014/50282-5, and 2017/10501-8 of São Paulo Research Foundation (FAPESP) and Natura Cosméticos S.A. also do not represent conflict of interest.'

\section{MANAGEMENT OF A CONSECUTIVE SERIES OF MONOCHORIONIC TWIN PREGNANCIES COMPLICATED BY TWIN ANEMIA: POLYCYTHEMIA SEQUENCE (TAPS) AT A SINGLE CENTER}

Mariano M. Lanna, Stefano Faiola, 'Federica Fuse', Teresa Izzo, and Maria Angela Rustico

Fetal Therapy Unit 'U. Nicolini', Children's Hospital Buzzi, Milan, Italy

Introduction: Prenatal diagnosis of twin anemia-polycythemia (TAPS) sequence is based on Doppler velocimetry of the middle cerebral artery, with peak systolic velocity (PVS-MCA) $\geq 1.5$ multiples of median (MoM) in one twin and $\leq 1.0 \mathrm{MoM}$ in the co-twin, in absence of oligo-polyhydramnios sequence. TAPS sequence can occur as a spontaneous complication of monochorionic twin pregnancies or after laser coagulation of placental anastomosis for treatment of twin-to-twin transfusion syndrome (TTTS). Four stages are described, based on the progression of TAPS, and different management options are considered. The aim of this study is to analyze the management and outcome of a consecutive series of TAPS observed at a single center. Materials and Methods: A retrospective analysis of perinatal outcome of TAPS observed in our center during the period 2004-2016. Management of each case was tailored to the stage of severity. Treatment options were laser coagulation of placental anastomosis, intrauterine fetal transfusion (IUFT) of the anemic twin or, depending on gestational age (GA) and parental consensus, bipolar cord coagulation (BCC) of one twin. Results: There were 34 TAPS, 11 spontaneous and 23 post-laser, observed at a median GA of 22 weeks in both groups.Stage 1 occurred in 15 cases (44\%), and these were managed conservatively. Of the 19 remaining cases (thirteen stage 2 , four stage 3 , and two stage 4 ), a total of 7 (three stage 2 , three stage 3 , one stage 4 ) were managed actively with laser (5), IUFT (1) and BCC(1). Termination of pregnancy was chosen in one case. Spontaneous single fetal demise occurred in 7 out of $27(26 \%)$ untreated cases. No significant difference was observed between the treated and untreated groups in the rates of premature preterm rupture of membrane ( $28 \%$ vs. $11 \%, p=.39)$, GA at birth (30 and 31 weeks, $p=.20)$, or in overall survival rate $(10 / 14$, $71 \%$ vs. $38 / 54,70 \% p=.80$ ). In stages 2 and 3 , treatment improved outcome with an overall survival rate increasing from $55 \%$ in untreated cases to $75 \%$ after treatment. Neurological impairment at 
postnatal follow-up was found in only one untreated polycythemic twin. Conclusions: Untreated TAPS has a high incidence of fetal demise. Treatment in stages 2 and 3 can improve outcome with no effect on prematurity or neurological outcome.

\section{OUR TWO YEARS' EXPERIENCE WITH DIAGNOSTIC WHOLE EXOME SEQUENCING (WES) IN TWINS}

\author{
Petra Zwijnenburg', Quinten Waisfisz' , Hanne Meijers-Heijboer', Hans Gille', \\ and Dorret Boomsma ${ }^{2}$ \\ ${ }^{1}$ Department of Clinical Genetics, VU University Medical Center, Amsterdam, the \\ Netherlands \\ ${ }^{2}$ Netherlands Twin Register, Department of Biological Psychology, Vrije Universiteit, \\ Amsterdam, the Netherlands
}

Introduction: Recently, whole exome sequencing (WES) has become available as a diagnostic tool in mental retardation and/or congenital abnormalities. WES is a powerful approach for establishing the etiological diagnosis in those patients, but may also result in identifying a less expected cause or unsolicited findings. In order to recognize potential unique aspects of genetic counseling in twin pairs, we evaluated all genetic counseling consultations at the VUMC clinic in which WES has been performed. Materials and Methods: We evaluated all genetic counselings in which WES was performed between January 2015 and February 2017 on a twin. Results: WES was performed in 29 twins. Seven twins were unaffected parents, in whom WES was performed because of an affected child (trio analysis of both parents and the child). In 22 affected twins WES was performed; in 2 (concordant) twin pairs, DNA samples from whole blood from both twins were analyzed and in 18 twin pairs (14 discordant and 4 concordant) WES was only performed in one twin. In 11 cases WES identified a (possible) causative mutation, also including mutations with known reduced penetrance or variable expression. In two twins WES showed an unsolicited finding. In five twins, the identified mutation using WES was subsequently analysed by Sanger sequencing in the co-twin. In five samesex twin pairs zygosity was determined by DNA analysis either before or after WES. Evaluation of those cases illustrates several unique aspects of WES in twins: First, certainty about zygosity before WES is often helpful, in particular when a disease with reduced penetrance or variable expression is identified in the co-twin. Also, in most situations, in MZ twin pair WES in one twin (and parents) will be sufficient. We propose a flow chart for WES in same sex twins. Next, if a WES finding was subsequently investigated in the co-twin in blood-derived DNA, awareness of possible chimerism in blood is important. Furthermore, our experience illustrates the importance of being informed on zygosity, promoting understanding and decision making in the situation that one of the parents of an affected child included in WES analysis is a twin. Being a monozygotic twin may add another aspect to the complexity of potential unsolicited findings. Conclusion: Our first experiences highlight that it is important to be aware of unique aspects and pitfalls in genetic counseling in twins and the importance of being informed on zygosity in same sex twins.

\section{CASE REPORT OF ANTEPARTUM DEATH OF ONE TWIN}

Beatriz Pérez Munárriz, Lara Martínez Jimenez, Natalia Abian Franco, Maitane Urtasun Murillo, Sonia García Francés, and Carlos Larrañaga Azcárate

Gynecology and Obstetrics Department, Complejo Hospitalario De Navarra, Spain

Introduction: The death of one of the fetuses in a bichorionic pregnancy, although to a much lesser extent than in the monochorionic pregnancy, implies an increased risk for the surviving fetus, which includes: Death: 3\% (95\% CI [0.4, 5.7]); Preterm delivery: 54\% (95\% CI [41.5, 66.9]); Pathological neuroimaging: 16\% (95\% CI [7.8, 23.5]); Alteration of neurodevelopment: $2 \%$ (95\% CI [1.6, 4.9]). Case Report: We present the case of a twin pregnant woman of 32 weeks' gestation with normal course of pregnancy. The pa- tient was referred to gynecological urgencies for abdominal pain and was diagnosed of fetal death of the second twin. She was diagnosed of threat of preterm labor (she had contractions and cervical shortening). No signs of chorioamnionitis or coagulation alterations were found. Tocolytic treatment was started with atosiban for 48 hours and 2 doses of $12 \mathrm{mg}$ of betamethasone were administered to achieve fetal lung maturation. Additional treatment with nifedipine was necessary to control uterine dynamics. Magnesium sulfate was not administered as fetal neuroprotection because of its gestational age. Blood tests were performed with serologies for cytomegalovirus, parvovirus B19, syphilis being all normal. The patient was discharged from the hospital at week $32+6$ gestation and continued follow-up in the high-risk obstetric consultation. At week 36 of gestation, an emergency cesarean was performed due to the onset of spontaneous labor with podalic presentation of the first twin. The patient refuses to perform necropsy of the dead fetus. She had a favorable evolution in her postpartum stay in observation. The live newborn evolved favorably in his stay in the neonatology unit. He is currently a healthy child. Conclusions: In cases of antepartum fetal death parents should be informed of the risks that this new situation implies. Gestation control can be performed in the vast majority of cases of ambulatory control. The risk of coagulopathy is very unlikely in these pregnant women, so it is not considered necessary at the present time a special surveillance in this sense. If there is no complication, the management must be expectant until reaching the gestational age at term. In dichorionic twins, death of one twin is not, by itself, a strong indication for delivery of the surviving twin. However, if a condition affecting both twins is present (e.g., preeclampsia, chorioamnionitis), then close surveillance and timely delivery of the surviving twin are indicated to prevent a second fetal loss.

\section{MORTALITY AND MORBIDITY OF THE SECOND TWIN RELATED WITH DELIVERY MODE}

Paula Alonso, Virginia Domínguez, Santiago García-Tizón, Pilar Pintado, Laura Pérez, and Yolanda Cuñarro

Hospital General Universitario Gregorio Marañón, Madrid, Spain;

Introduction: The incidence of twin pregnancies has increased due to both use of assisted reproduction techniques (ART) and increased maternal age in the past 20 years. The second twin is the most affected one as a result of delivery trauma. The objective of this study was to analyze mortality and morbidity of the second twin and its relation to delivery mode. Methods: We performed a retrospective analysis from a cohort of 873 twin births assisted in Gregorio Marañon University Hospital between 2012 and 2016. Data were collected from wards clinical history. Results: The mean gestational age at delivery was 35 weeks, $83 \%$ were dichorionic diamniotic and $58 \%$ were obtained from ART. Regarding delivery mode $51 \%$ were vaginal and $49 \% \mathrm{C}$-section. There were no significant differences in mortality rate between the first and the second twin (1.7 vs. 2.1, $p=.8$ ), nor between vaginal delivery and C-section for both twins (3.7 vs. $2.4, p=.08$ ). However, when we analyzed the mortality rate between vaginal delivery and cesarean stratified by gestational age, mortality was significantly higher for vaginal delivery below 32 weeks (46 vs. $11.9, p<.01$ ) but there were no significant differences after 32 weeks ( 1.1 vs. $1, p=.56)$. Morbidity was defined by $\mathrm{pH}$ at birth less than 7 and NICU admission requirement. Regardless of delivery mode, second twins needed more NCIU admission than first ones ( 34.7 vs. $41.8, p=.02$ ), but there were no significant differences in low $\mathrm{Ph}(0.6$ vs. $1.2, p=.5)$. When the second twin was not cephalic, low $\mathrm{pH}$ was only found in vaginal deliveries compared to C-section ( $1.8 \%$ vs. $0 \%, p=.01)$; but we did not find significant differences for NCIU admission between vaginal and cesarean delivery(38.8 vs. $47.4, p=.17$ ). We did not find differences in morbidity for vaginal delivery of the second twins when they were cephalic or not cephalic ( $\mathrm{pH}<70.7$ vs. $1.8, p=.6$; NICU admission 31.4 vs. $38.8, p=.21)$. Discussion and Conclusion: Election 
criteria regarding mode of delivery for twins in our center is the same as recommended by both Spanish and American guidelines. The results obtained in this study are consistent with the literature. To conclude, despite of an increased second twin morbidity, it does not seem to be related to delivery mode if the election criteria for vaginal delivery or $\mathrm{C}$-section is properly indicated.

\section{ACADEMIC ACHIEVEMENT OF TWINS BASED ON PLACE OF RESIDENCE AND TYPE OF EDUCATIONAL ESTABLISHMENT}

\section{Marina Egorova', Yulia Chertkova', and Anna Fominykh²}

${ }^{1}$ Department of Psychology, Lomonosov Moscow State University, Moscow, Russian Federation

${ }^{2}$ Psychological Institute of Russian Academy of Education, Moscow, Russia

This article examines the effect of inter-family environment factors on the academic achievement of twins in the Russian Federation. The factors considered include the prosperity of the region of residence (gross regional product, or GRP, per capita), the type of settlement where student attended school (megalopolis, city, rural), and the type of educational establishment (ordinary public schools vs. advanced learning schools). Achievement was assessed based on Unified State Exam (USE) scores in the Russian Federation in 2010-2012. Students could take tests in 14 subjects, two mandatory (Russian and mathematics) and 12 optional (English, German, French, Spanish, history, literature, physics, chemistry, biology, geography, information and communication technologies, and social studies). The USE is scored on a scale of 0 to 100 , where 100 is the top score. Data on the USE scores of 11,577 twin pairs $(13,214$ girls and 9,940 boys) were received. The sample was representative of the twin population of relevant age residing in the Russia. Twins accounted for about $0.9 \%$ of all school graduates who took the USE. The data show that the academic achievement of twins upon graduation from school is not inferior to that of singletons. Prosperity in the region of residence did not exhibit a great effect on the academic achievement of twins. Spearman's correlation coefficients for GRP per capita and USE scores did not exceed 0.10 for mandatory subjects and 0.17 for optional subjects. Students from cities performed better on the USE than students from rural areas. There were only two subjects out of fourteen (history and geography) where a significant difference between the scores of urban and rural students could not be observed. Graduates of advanced learning schools did better in all subjects than graduates of ordinary public schools, which may reflect the selective admissions process and/or a better quality of teaching at advanced learning schools. Twin intrapair similarity did not correlate with regional prosperity, but it did exhibit links with the size of the settlement and the type of educational establishment. There is less intrapair similarity among students from cities and students from gymnasiums, presumably due to greater environmental variation and individualization of development. On the whole, one can say that economic factors have a lesser effect on the academic achievement of twins than the type of settlement or the type of educational establishment.

\section{TRANSABDOMINAL CERCLAGE IN TWINS: SERIES OF SEVEN CASES}

Frederic Debieve, Patricia Steenhaut, and Corinne Hubinont

Obstetric Department, Cliniques Universitaires Saint Luc, Brussels, Belgium

Background: Cervical insufficiency is an inability of the uterine cervix to retain a pregnancy in the second trimester, in the absence of clinical contractions, labor, or both. The diagnosis is based on history of recurrent second or early third trimester. The incidence of cervical insufficiency among pregnant women is 0,5 to $1 \%$, but can be as high as $75 \%$ among women with preterm birth. Transvaginal cerclage is the common treatment of cervical insufficiency. However, TVC has several limits, especially in women with very short cervix and in twin pregnancies. In those patients, a transabdominal cerclage appears to be a very effective procedure, as it is placed at the cervico-isthmic junction. Nevertheless, transabdominal cerclage remains controversial. Aim: to evaluate the outcomes of transabdominal cerclage in twin pregnancies in terms of preterm birth rate and neonatal morbidity and mortality. Materials and Method: We conducted a retrospective unicenter study of seven patients with twin pregnancies who underwent transabdominal cerclage at the end of first trimester (12-15weeks). We selected patients who met the indications of TAC, which are a history of TVC failure or a cervix too short to allow TVC with history of fetal loss. The antenatal and delivery data of the selected patients were collected and compared to those of their previous pregnancy. Outcomes: All patients carried their pregnancy throughout second trimester and delivered 100\% living newborns during the third trimester. Mean gestational age was $321 / 7$ weeks and mean birth weight was $2124 \mathrm{~g}$. Neonatal morbidity rate was $50 \%$, which was mostly related to preterm birth. Mean duration of neonatology stay was 32 days. As for the surgical procedure of TAC placement, no operative complications were reported. Conclusions: Perinatal outcomes were considerably improved after transabdominal cerclage. Our findings corroborate those of previous case reports and support the efficacy of TAC in twin pregnancies.

\section{THE SIGNIFICANCE OF PLACENTAL CORD INSERTION SITE IN TWIN PREGNANCY}

Erkan Kalafat, Basky Thilaganathan, Aris Papageorghiou, Amar Bhide, and Asma Khalil

Fetal Medicine Unit, St George's Hospital, St George's University of London, Cranmer Terrace, London, UK

Objective: The aim of this study was to investigate the association between abnormal cord insertion and the twin-specific complications, including birthweight discordance (BWD), sFGR and TTTS Methods: A single-center cohort study of twin pregnancies. Information on the pregnancies, ultrasound findings, prenatal investigation and interventions, placental histopathological findings, pregnancy outcomes, birthweight and gestation at delivery were obtained from the maternity records. Categorical variables were compared by the $\chi^{2}$-test, while continuous variables were compared using the $t$ test, ANOVA for multiple comparison and the Kruskal-Wallis test. Results: 497 twin pregnancies, 351 (70.6\%) DC and 146 (29.3\%) $\mathrm{MC}$, were included in the analysis. The incidence of BWD of $25 \%$ or more was significantly higher in pregnancies with velamentous and marginal cord insertions compared to those with normal cord insertion $(24.5 \%, 15.2 \%$ vs. $7.5 \%, p<.001$ and $p=.020$ respectively). In pregnancies with BWD of $25 \%$ or more, the smaller twins had significantly higher prevalence of velamentous (13.8\%) and marginal $(34.2 \%)$ cord insertions compared to the larger twins $(1.8 \%$ and $18.5 \%$, respectively $p<.001)$. The smaller twins of the MCDA pregnancies showed an even higher prevalence of velamentous $(29.5 \%)$ and marginal cord insertions $(40.9 \%)$ compared to the larger twins $(2.3 \%$ and $29.5 \%$ respectively $p<.001)$. Compared to the normal cord insertion group, only velamentous insertion was significantly associated with the risk of sFGR (OR 9.24; 95\% CI [2.05, 58.84], $p<.001$ ), BWD of $20 \%$ or more (OR 4.34; $95 \%$ CI $[1.36,14.61], p=.006)$ and $25 \%$ or more (OR $6.81 ; 95 \%$ CI $[1.67,34.12], p=.003)$ in MC twin pregnancies. There was no significant association between velamentous cord insertion and TTTS $(p=.265)$. There was no significant association between marginal cord insertion and the development of sFGR $(p=.233)$, BWD of $25 \%$ or more $(p=.114)$ or TTTS $(p=.487)$. Subgroup analysis of DC twins showed that abnormal cord insertion was not associated with the risk of BWD $(p=.250)$, sFGR $(p=.308)$, composite neonatal adverse outcome $(p=.637)$ or intrauterine death $(p=$ .349). Conclusion: MC twins with velamentous cord insertion are at increased risk of BWD and SFGR. Sonographic delineation of the cord insertion could be of value in the antenatal stratification of twin 
pregnancies. Prospective studies are required to assess the value and the predictive accuracy of this potential screening marker.

\section{MANAGEMENT DIFFICULTIES IN THE CASE OF A MAJOR MORPHOLOGICAL ABNORMALITY IN ONE OF THE TWINS}

Assaad Kesrouani', Inaam Hatoum', Elie Choueiry ${ }^{2}$, Bernard Nasr ${ }^{3}$, Linda Daou ${ }^{4}$, Elie Attieh', and Ramzi Finan'

${ }^{I}$ Obstetrics and Gynecology Department, St Joseph University, Beirut, Lebanon

${ }^{2}$ Pediatrics Department, St Joseph University, Beirut, Lebanon

${ }^{3}$ Fetal Care Center, Beirut, Lebanon; 4. Pediatrics Department, Cardiopediatrics, St Joseph University, Beirut, Lebanon

Introduction: Many challenges are present in the case of prenatal abnormality in a fetus. The difficulty is increased in case of twins where difficult decisions face the parents and the physician. We report 6 cases that have in common a major abnormality in one of the fetus. Case Series: From 2009 to 2016, our retrospective study includes 6 cases of morphological abnormality in one the fetuses, the other having a normal follow-up. These cases included: (1) posterior encephalocele with a single umbilical artery; (2) thoragopagus twins aside a normal fetus; (3) A spina bifida; (4) Multiple anomalies including pyelectasis, clubfoot and cleft palate; (5) Two cases with cardiac malformation (Right ventricular hypoplasia with VSD - A complex cardiac malformation). All cases had a prenatal ultrasound diagnosis, at a mean age of 17 weeks. Three pregnancies resulted from IVF. Couples were informed about the possibility of invasive diagnostic procedures and about the interruption of the pregnancy involved. Issued discussed by the couple included ethics, religion, preterm delivery, the relevance of invasive procedure, postnatal outcome, and risk for the other baby. All couples chose not to do an amniocentesis. Only one couple chose interruption of pregnancy (thoragopagus twin). Delivery was done at a mean term of 34 weeks. Conclusion: Management of a twin pregnancy with a major abnormality in one fetus includes many challenges as couples require explaining the many facets of the invasive procedure and they often are reluctant for doing it.

\section{NEONATAL AND MATERNAL OUTCOMES IN SPONTANEOUSLY-CONCEIVED TWIN PREGNANCIES ACCORDING TO MODE OF DELIVERY}

Samantha McKenzie Stancu and Manuela Cristina Russu

St. Mark's Hospital and Academic Institute NHS Foundation Trust, London, UK and Dr. I. Cantacuzino Clinical Hospital, Bucharest, Romania

Introduction: Appropriate intrapartum conduct in a twin delivery remains a challenging aspect of obstetric practice. The objective of this study was to compare neonatal and maternal outcomes in twin pregnancies according to mode of delivery. Materials and Methods: This is a single centre retrospective cohort study of all consecutive spontaneously-conceived twin deliveries ( $\geq 24$ weeks, estimated fetal weight $\geq 500$ grams) between 01/01/2007-31/12/2016 at a tertiary-level centre. Neonatal outcomes included survival, Apgar score, prematurity-associated pathology (PAP), admission to the neonatal intensive care unit (NICU) and length of stay (LOS). Maternal outcomes included postpartum complications and LOS. Statistical analysis encompassed both univariate and multivariate statistics, the latter comprising chi-square test with subsequent $p$ value and odds ratio with $95 \%$ confidence interval. Statistical significance was set at $p<.05$. Results: A total of 173 consecutive women with spontaneously-conceived twin deliveries were enrolled in this study. One-hundred and twenty-nine $(74.6 \%)$ women delivered by cesarean section (CS). The success rate of vaginal delivery (VD) was $93.6 \%$ (44/47). The proportion of low and critically-low Apgar scores in neonates delivered by CS and VD was similar, $11.6 \%$ and $11.3 \%(p=.49)$, respectively. Birth weight was significantly lower among neonates delivered by CS $(p=.0017)$. A strong statistical correlation was identified between CS and neonatal intensive care unit (NICU) admission; $53.2 \%$ vs. $1.5(p=.0001)$. Neonatal LOS in the NICU was significantly longer in the CS group; range: 1-68 days, average: 22.78 days compared to the VD group: 1-30, 12.05 days). Prematurity-associated pathology was noted in 75 pairs of twins (75/173); 61 pairs were delivered by CS, bearing strong statistical significance $(p<.0001)$. Postpartum complications occurred in $14.7 \%$ of CS compared to $13.6 \%$ of VDs $(p=.85)$. Maternal LOS was longer in women who delivered by CS; range: 4-83 days, average: 15.65 compared to 3-83, 12.13 following VD. Conclusions: The success rate of VD was $93.6 \%$. Neonates delivered by CS had a higher rate of NICU admission, PAP, longer LOS and lower birth weight. None of the cases involved intrapartum complications or intrapartum fetal death. In conclusion, this study showed that VD is safe, especially when the first twin is in cephalic presentation.

\section{TRENDS IN THE PREVALENCE OF TWIN PREGNANCIES AND ROUTE OF DELIVERY IN A TERTIARY CARE CENTER IN LEBANON}

Assaad Kesrouani ', Inaam Hatoum' ', lyad Mallak ${ }^{2}$, Norma Aouad ${ }^{3}$, Elie Choueiry ${ }^{4}$, Linda Daou ${ }^{4}$, Elie Attieh', and Ramzi Finan'

${ }^{1}$ Obstetrics and Gynecology Department, St Joseph University, Beirut, Lebanon ${ }^{2}$ Faculty of Medicine, St Joseph University, Beirut, Lebanon

${ }^{3}$ Registered Midwife, Hotel-Dieu de France University Hospital, Beirut, Lebanon ${ }^{4}$ Pediatrics Department, St Joseph University, Beirut, Lebanon

Introduction: Over the years there seems to be an increase in twin pregnancies mainly due to use of assisted reproductive techniques, but the optimal route of delivery of these pregnancies is not yet defined. Despite an increasing tendency toward cesarean delivery of twin pregnancy, some patients demand a normal delivery. The aim of this study was to investigate the change in the prevalence of twin pregnancies and to evaluate their mode of delivery over a 14-year period, in order to define the current trends in clinical practice regarding twin deliveries in Lebanon. Material and Methods: This was a retrospective, observational cohort of pregnancies delivered at a single tertiary care center in Beirut from 2002 to 2016. Patients included were above 24 weeks at delivery. We analyzed the evolution in the prevalence and the route of delivery of twin pregnancies over time. The data collected were analyzed using the JonkheereTerpstra test. The significance level was 0.05. Results: There were 14,190 infants born at a tertiary care center from 2002-2016. Of these, 384 deliveries were twin births. The proportion of twin pregnancies did not change over time $(p=.618)$. This is surprising in the light of the more frequent use of IVF and ovarian stimulation in this time period. The proportion of cesarean delivery and vaginal delivery remained stable over the 14-year period $(p=.149$ and $p=.149$ respectively). Cesarean section is the main route of delivery in most of twin pregnancies. The changes in the prevalence and the route of delivery over years are attributable to random variation. Conclusion: The prevalence of twin pregnancies remains stable over time. We found no change in the evolution of the route of delivery over 14 years, the cesarean delivery being the main route of delivery. These trends may reflect a health care issue that deserves further research.

\section{THE EFFECTIVENESS OF ARABIN PESSARY IN TWIN} PREGNANCY

Olesya Bespalova' and Gabriel Sargsyan 1,2

${ }^{I}$ FSBSI, The Research Institute of Obstetrics, Gynecology and Reproductology named after D.O.Ott, St. Petersburg, Russia

${ }_{2}^{2}$ SBHCI, Tosno Clinical Interdistrict Hospital, Tosno, Russia

Introduction: Preterm birth (PB) represents an unsolved challenge in modern obstetrics, particularly due to the increasing rates of twin pregnancy (TP), a well-known risk factor for $\mathrm{PB}$, with the widespread introduction of assisted reproductive technologies. We aimed to evaluate the effectiveness of Arabin obstetric pessary for $\mathrm{PB}$ in women with TP and untimely ripening of the cervix. Materials 
and Methods: Sixty-four women with TP and short cervix $(\leq 38 \mathrm{~mm}$ (4.5, sanitized. Pessary was removed prematurely in the occurrence of premature rupture of membranes (PROM), vaginal bleeding, or with development of labor. In the absence of indications for early removal, the obstetric pessary was removed at 37 weeks of gestation. Results: In this group, $53(82.8 \%)$ had dichorial diamniotic twins, and $11(17.2 \%)$ had monochorial diamniotic twins. In $48(75.0 \%)$ cases, pregnancy occurred after in vitro fertilization. Fifty $(78.1 \%)$ women suffered from infertility - primary in $32(50.0 \%)$, and secondary in $18(28.1 \%)$. A high level of somatic $(59.4 \%)$ and gynecological pathology $(81.2 \%)$ was present among the included women. After the installation of Arabin pessary, on average, the duration of pregnancy was prolonged with $10,4 \pm 0,7$ weeks, $14,2 \pm 0,3$ weeks for term deliveries, and 8,1 $\pm 0,5$ weeks for PBs. Labor was complicated with PROM in 19 (29.7\%) pregnant women. After removal of the pessary at the 37 weeks of gestation, the interval to delivery was on average 5.6 days. In $33(51.5 \%)$ pregnant women, delivery occurred after 37 weeks of gestation (term delivery). PB occurred in $31(48.4 \%)$ women, of whom $<34$ weeks in $9(14,1 \%)$, between 34 and 36 6/7 weeks in $22(34,4 \%)$; no births occurred $<28$ weeks of gestation. Conclusion: The use of Arabin obstetric pessary in twin pregnancy is an effective and safe method that allows prolonging pregnancy, reducing the occurrence of PB and improving perinatal outcomes.

\section{INVESTIGATING THE GENETIC BASIS OF SINGING ABILITY: A PILOT ONLINE TWIN STUDY}

Yi Ting Tan 1,4, Gary E. Mcpherson ', Isabelle Peretz ${ }^{2}$, Sam F. Berkovic ${ }^{3}$, and Sarah J Wilson ${ }^{3,4}$

${ }^{I}$ Melbourne Conservatorium of Music, University of Melbourne, Parkville, Victoria, Australia

${ }^{2}$ International Laboratory for Brain, Music and Sound Research and Department of Psychology, Université de Montréal, Montreal, QC, Canada

${ }^{3}$ Department of Medicine, Epilepsy Research Centre, University of Melbourne,

Heidelberg, Victoria, Australia

${ }^{4}$ Melbourne School of Psychological Sciences, University of Melbourne, Parkville, Victoria, Australia

Introduction: For centuries, many have been intrigued by the expression of musical abilities. The past decade has seen an increase in behavioral and molecular genetic studies investigating various music behaviors such as music perception abilities and music creativity. While some promising and converging genetic evidence have emerged, the genetic basis of singing ability has received minimal research attention thus far, despite singing being a universal musical trait which emerges spontaneously in infancy even prior to formal music training. Materials and Methods: A pilot twin study involving 108 twin pairs (70 MZ; $38 \mathrm{DZ}$ ) was conducted using a novel, purpose-built online program 'Let's Hear Twins Sing!' with online recording capability. Singing ability (in terms of pitch accuracy) was objectively assessed by three different singing tasks (i.e., singing a familiar song, imitating single pitches, and imitating short melodies), and participants also provided ratings of their singing ability. Environmental factors associated with singing ability were also investigated using a questionnaire on music and singing experience. Results: Univariate genetic modelling demonstrated that in this pilot sample, additive genetic variance was more important than shared and unshared environmental variances in explaining the variation in singing ability, both for singing pitch accuracy measured across all the singing tasks $(A=69 \% ; C=11 \% ; E=19 \%)$, as well as self-rated singing ability $(\mathrm{A}=72 \% ; \mathrm{C}=13 \% ; \mathrm{E}=15 \%)$. Significant additive genetic components were also estimated for several environmental factors associated with singing ability: instrumental expertise $(A=68 \%)$, years of music training $(A=46 \%)$, and singing in public $(A=66 \%)$. Bivariate genetic analyses revealed that the associations between singing ability and both instrumental expertise and years of music training were mediated significantly by shared additive genetic influences. Conclusions: Pilot study find- ings provide preliminary evidence for the role of genes in influencing singing ability and several associated environmental factors. A larger twin sample is necessary to provide more robust estimations of genetic and environmental influences on singing ability. To this end, we are conducting a large online twin study using an innovative cross-platform HTML5 web application and we plan to extend our participant recruitment internationally.

\section{MOST FREQUENT COMPLICATIONS IN TWIN PREGNANCIES - EXPERIENCE OF A PORTUGUESE PUBLIC HOSPITAL}

Inês Morais Rodrigues, Inês Vermelho Lourenço, Helena Gomes, Diana Martins, Elsa Dias, and Carlos Verissimo

Hospital Beatriz Ângelo, Loures, Lisboa, Portugal

Introduction: Twin pregnancy associates with maternal and perinatal complications. Hypertensive complications are more frequent in theses pregnancies than in singleton pregnancies. Among other complications, prematurity, preterm premature rupture of membranes (PPRM) and fetal growth restriction (FGR) can be mentioned. The aim of this work was to characterize the population of women with twin pregnancies and evaluate the more frequent maternal/perinatal complications. Materials/Methods: retrospective study of all twin pregnancies that delivered in a Portuguese hospital, Hospital Beatriz Ângelo, from 2013 to 2016. Revision of all clinical records allowed to evaluate age, parity, type of conception and chorionicity. The five more frequent maternal (PIH/preeclampsia, Gestational Diabetes) and perinatal (threat of premature birth, premature birth and FGR) complications were analyzed. Results: Average age of these pregnants $(n=121)$, was 31.9 years (18-45). Approximately half $(63 / 121)$ were nulliparous and the majority concerned spontaneous conception (106/121) and bichorionic/biamniotic pregnancies (93/121). The most frequent complication was prematurity (73/121), associated with PPRM in $23 \%$ of the cases. It consisted frequently of deliveries after 34 weeks (58/73). Other frequent perinatal complications were threat of premature delivery $(18 / 121)-$ $39 \%$ of which with delivery before 34-weeks; and FGR (17/121) - about one third were monochorionic (5/17) and in more than half (10/17) this was the reason for termination of pregnancy before 37 weeks. Hypertensive disorders were the most frequent maternal complication (PIH 5/121 and preeclampsia 12/121). Although PIH occurred earlier (in a 29-week pregnancy compared to preeclampsia in a 32-week pregnancy), preeclampsia determined end of pregnancy sooner (32 vs. 34-weeks of pregnancy). Gestational diabetes mellitus was also a frequent maternal complication (12/121), all cases diagnosed in the second trimester. Only one case associated with one fetus with fetal growth superior to the 90th percentile. Conclusions: Twin pregnancies revealed high prevalence of hypertensive complications as stated in literature. Prematurity is an important complication, although most of the cases consist of late preterms. FGR is frequent and motivates part of the premature deliveries.

\footnotetext{
HERITABILITY OF COLD AND HEAT PATTERNS

Yoon-Mi Hur', Hana $\mathrm{Yu}^{2}$, Hee-Jeong Jin ${ }^{2}$, and Siwoo Lee ${ }^{2}$

${ }^{1}$ Research Institute for Welfare Society, Mokpo National University, Jeonnam, South Korea

${ }^{2}$ Mibyeong Research Center, Korea Institute of Oriental Medicine, Daejeon, South Korea
}

Introduction: Although there have been growing interests in the role of the Cold and Heat Patterns (CHPs) of the patients in the diagnosis and treatment outcomes of various diseases, the genetic and environmental etiology of the CHPs remains largely unknown. The purpose of the present study was to investigate heritability of CHPs with a special emphasis on sex differences. Materials and Methods: As part of the South Korean Twin Registry project, over 1,800 adolescent and young adult twins completed a telephone interview for CHPs. 
Twins' zygosity was determined by the questionnaire method. Twin correlations were computed. Using Mx, we applied a scalar sexlimitation model to the raw twin data. Results: Consistent with prior studies, the Cold Pattern (CP) was higher in females than in males, whereas the Heat Pattern (HP) was higher in males than in females. Also, HP was significantly positively associated with BMI, especially in males. MZ twin correlations were significantly higher than DZ twin correlations for HP and CP in both sexes. Model-fitting analysis indicated that males had higher heritability than did females for both CP and HP.

HP showed significant nonadditive genetic influences especially in males. Conclusions: Both CP and HP are heritable phenotypes. In the future, genetic and environmental correlations between these phenotypes and various diseases will be investigated to better elucidate the etiologies of the diseases.

\section{SEX DIFFERENCE IN GENETIC AND ENVIRONMENTAL INFLUENCES ON SOMATIZATION SYMPTOMS}

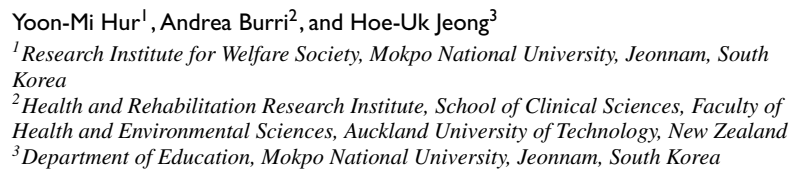

Introduction: A somatic symptom disorder is any mental disorder that manifests as physical symptoms that suggest illness or injury, but which cannot be explained fully by a general medical condition or by the direct effect of a substance or by another mental disorder. The prevalence rates of the somatic symptom disorder have been reported to be higher in females than in males (about $2 \%$ vs. $0.2 \%$ ). The present study aimed to explore heritability of somatization symptoms in a general population sample with a special emphasis on sex differences. Materials and Methods: Approximately 1,800 adolescent and young adult twins drawn from the South Korean twin registry were interviewed to assess the somatization symptoms. Zygosity was determined using a questionnaire method. Results: Consistent with previous studies, females showed higher levels of the symptoms than did males. MZ twin correlations were significantly higher than DZ twin correlations for the somatization symptoms in both sexes. Using Mx, we applied a scalar sex-limitation model to the data. The model-fitting analysis yielded moderate heritability in somatization symptoms in South Korean twins. Females had higher heritability than did males. Conclusions: There were sex differences in heritability of somatization symptoms (females $>$ males). Gender differences should be taken into account in treatments for the somatization symptoms.

\section{LEISURE-TIME PHYSICAL ACTIVITY SHOWS NO EFFECT ON} DNA METHYLATION AGE IN A DISCORDANT TWIN STUDY

\author{
Elina Sillanpää ', Miina Ollikainen ${ }^{2,3}$, Jaakko Kaprio ${ }^{2,3}$, Timo Törmäkangas ${ }^{\text {, and }}$ \\ Urho Kujala ${ }^{4}$ \\ ${ }^{I}$ Gerontology Research Center, Faculty of Sport and Health Sciences, University of \\ Jyväskylä, Jyväskylä, Finland \\ ${ }^{2}$ Institute for Molecular Medicine (FIMM), Helsinki, Finland \\ ${ }^{3}$ Department of Public Health, University of Helsinki, Helsinki, Finland \\ ${ }^{4}$ Faculty of Sport and Health Sciences, Exercise Medicine, University of Jyväskylä, \\ Jyväskylä, Finland
}

Introduction: DNA methylation (DNAm) age is a novel marker of biological age, which at the population level correlates highly with chronological age. DNAm age acceleration describes the discrepancy between chronological age and DNAm age i.e. if a person is biologically younger or older compared with his/her chronological age. High DNAm age acceleration predicts higher mortality risk. Materials and Methods: We performed quantitative genetic modeling in young (20- to 25-year-old) and older (55- to 75-year-old) monozygotic (MZ, $n=168$ young, $n=122$ older) and dizygotic
(DZ, $n=121$ young, $n=42$ older) twin pairs to investigate the contributions of genetic and shared and non-shared environmental variation on age acceleration. Age acceleration (residuals from a linear regression model of DNAm age on chronological age) in white blood cells was calculated from methylation data using an online calculator (https://dnamage.genetics.ucla.edu). Thereafter, we tested the hypothesis that leisure-time physical activity is one of the non-shared environmental factors that affect epigenetic aging. We performed a co-twin control analysis with same-sex twin pairs who had persistent discordance in leisure-time physical activity for 32 years. 16 twin pairs (seven MZ and nine DZ pairs, mean age 60.4 years) of which the same co-twin had a higher leisure time-activity volume at the majority or all of the follow-up time points according to reported/interviewed physical-activity data participated in the study. Results: More of the variation in DNA methylation based age acceleration was explained by non-shared environmental factors among older twin pairs $47(35,63) \%$ compared with younger pairs $26(19,35) \%$, difference between age groups $p<.001)$. The proportions accounted for genetic variation were $26(19,35) \%$ and 74 $(65,82) \%$ respectively. Despite of multiple phenotypic differences associated with leisure-time physical activity throughout adult life, DNAm age did not differ among active and inactive co-twins, being 60.7 vs. 61.8 years, respectively (between groups mean-difference: 1.17 (95\% CI [3.43,1.10]). Conclusions: Results suggest that leisuretime physical activity during adult years has at most minor effects on epigenetic aging. This supports recent findings that long term leisure-time physical activity in adulthood has little or no effect on mortality after controlling for genetic factors.

\section{IS THE ASSOCIATION BETWEEN OBESITY AND HIP OSTEOARTHRITIS EXPLAINED BY FAMILIAL CONFOUNDING? RESULTS FROM THE NOR-TWIN OA STUDY}

Karin Magnusson, Katrina Scurrah, Ragnhild Elise Ørstavik, Thomas Sevenius Nilsen, Ove Furnes, and Kåre Birger Hagen

${ }^{1}$ National Advisory Unit on Rehabilitation in Rheumatology, Department of Rheumatology, Diakonhjemmet Hospital, Oslo, Norway

${ }^{2}$ Clinical Epidemiology Unit, Orthopaedics, Department of Clinical Sciences Lund, Lund University, Lund, Sweden

${ }^{3}$ Australian Centre of Excellence in Twin Research, Centre for Epidemiology \& Biostatistics, University of Melbourne, Melbourne, Australia

${ }^{4}$ Norwegian Institute of Public Health, Oslo, Norway

${ }^{5}$ Department of Orthopaedic Surgery, Haukeland University Hospital, Bergen, Norway ${ }^{6}$ Department of Clinical Medicine, Institute of Medicine and Dentistry, University of Bergen, Norway

Introduction: To study whether familial confounding due to genetics or environmental factors explains the association between Body Mass Index (BMI) and severe hip osteoarthritis (OA). Materials and Methods: Data from the Norwegian Arthroplasty Registry were linked with the Norwegian Twin Registry in 2014, generating a population-based prospective cohort study of same-sex twins born 1915-60 (53.4\% females). BMI was calculated from self-reported height and weight. The outcome was incident hip arthroplasty due to OA (follow-up time: 1987-2014, 424,914 person-years). We performed sex-specific co-twin control analyses of dizygotic (DZ, $N=$ $5,226)$ and monozygotic (MZ, $N=3,803$ ) twin pairs using Cox regression models and compared the findings to those of cohort analyses. A weaker/non-significant association within MZ pairs compared to the cohort association would provide evidence of familial confounding. Results: The mean (SD) BMI was 22.6 (2.96), peak lifetime BMI 25.6 (2.61) and $N=614$ had hip surgery due to OA. In cohort analyses, BMI was associated with hip OA for women and men (Hazard Ratio $[\mathrm{HR}]=1.09,95 \%$ confidence intervals, $95 \%$ CI $[1.06,1.11]$ and HR $=1.08,95 \%$ CI $[1.04,1.12]$, respectively). When adjusting for familial confounding in within MZ twin analyses, the association got stronger for women (HR $=1.19,95 \%$ CI $[1.05,1.36])$ but weaker for men $(\mathrm{HR}=0.93,95 \%$ CI $[0.75$, 1.16]). Conclusion: The association between BMI and hip OA was 
likely non-causal and explained by familial confounding for men. For women, there was no evidence for familial confounding, consistent with a causal association.

\section{THE TWINS RESEARCH AUSTRALIA HEALTH AND LIFESTYLE QUESTIONNAIRE}

\begin{abstract}
Tessa L. Cutler, John L. Hopper, and Katrina Scurrah
Twins Research Australia, Melbourne School of Population and Global Health, The University of Melbourne, Melbourne, Australia
\end{abstract}

Introduction: In 2014 Twins Research Australia (TRA) began administering an online Health and Lifestyle Questionnaire (HLQ) to members. The questionnaire contained demographic questions and covered a broad range of health conditions and risk factors. It aimed to enable research by (1) allowing TRA to better describe its membership; (2) enabling identification of potential participants for specific research studies to reduce costs to researchers and reduce unnecessary approaches to ineligible TRA members; and (3) fast-tracking research by providing de-identified data to researchers for ethically approved research analyses. Methods: The design of the HLQ incorporated advice from twin researchers and validated questionnaires were used where possible. Many of the demographic and health questions were selected to enable comparison with Australian Bureau of Statistics data. All parents of twin pairs aged 1-18 and twins aged 18 or above were eligible to participate in the HLQ and were invited to complete the 20 minute online questionnaire. New members continue to be approached. Results: Over 6,700 adult twins, including over 1,800 pairs and over 3,600 parents of twin children have completed the HLQ. The responses to the survey have provided information on the zygosity of each pair from three sources; self-report, DNA test result and validated zygosity questionnaire. The results include lifestyle factors and current medical conditions for each twin which allowed analysis of the incidence as well as concordance within pairs. Examples of information obtained included self-reported health rating, diabetes, asthma, epilepsy, psychological wellbeing, mental illness, cancer, vision problems, smoking and alcohol consumption. Conclusions: Responses to the HLQ have allowed identification of over 1,000 twin pairs with specific medical conditions to meet the eligibility requirements of several studies recruiting through TRA. With this information, TRA has been able to use targeted approach methods to invite participants, allowing more participants to be recruited in less time. Identification of appropriate twin pairs has also provided important information for funding applications, both in Australia and internationally. Data from the HLQ has already been provided to researchers for several national and international projects either as unaccompanied de-identified data or as a linked dataset for analyses.

\section{CIGARETTE SMOKING, CIRCULATING LEVELS OF IMMUNE AND INFLAMMATORY MARKERS AND EPIGENTICS IN MONOZYGOTIC TWINS}

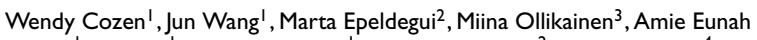
Hwang', Yang Yu' ', Ann S. Hamilton', Larry Magapantay ${ }^{2}$, Rachel Tyndale ${ }^{4}$, Thomas M. Mack', Otoniel Martinez-Maza ${ }^{2}$, David V. Conti' , and Jaakko Kaprio ${ }^{4}$ ${ }^{1}$ Keck School of Medicine of USC, University of Southern California, Los Angeles, California, USA

${ }^{2}$ David Geffen School of Medicine, University of California at Los Angeles (UCLA), Los Angeles, California, USA

${ }^{3}$ University of Helsinki, Helsinki, Finland

${ }^{4}$ University of Toronto, Toronto, Ontario, Canada

Introduction: Tobacco smoking can cause an inflammatory response, which may play a key role in the development and progression in smoking-related diseases. However, data regarding a comprehensive evaluation of the systemic immune perturbation caused by smoking is limited. We investigated the association between smoking, DNA methylation and circulating levels of immune/ inflammatory markers in serum samples from healthy monozygotic
(MZ) twin pairs discordant for smoking exposure. Materials and Methods: $67 \mathrm{MZ}$ twin pairs were identified from the Finnish Twin Cohort Study. We identified subsets of smoking discordant and concordant pairs from a group who had participated in clinical evaluations. Cotinine and 3hydroxycotinine were measured from the serum using LC-MS/MS. 25 immune/inflammatory biomarkers were measured using a Luminex platform $(R \& D$, Minneapolis, MN). DNA Methylation was assessed using the Infinium HumanMethylation 450K BeadChip array (Illumina, Inc. San Diego, CA) and beta values calculated for each locus for each twin. Current smokers were defined by cotinine $>3.08 \mathrm{ng} / \mathrm{ml}$ (Benowitz et al. AJE, 2009), with light vs. heavy smokers categorized using the median cotinine level $(78.17 \mathrm{ng} / \mathrm{ml})$ among smokers. Questionnaire reports included amount (cigarettes per day, CPD), duration (years smoked) and years since quitting. We used linear mixed models, adjusted for age and sex to assess the association between the smoking variables and serum levels of each individual biomarker, and between the DNA methylation loci and biomarker levels, accounting for multiple comparisons. Results: Smokers had significantly higher levels of B-cell activating factor (BAFF) and thymus and activation-regulated chemokine (TARC) compared to non-smokers, with a dose-response by level of smoking, measured by CPD $(p=.046$ and $p=.044$, respectively) or years of smoking ( $p=.011$ and $p=.005$, respectively). Higher cotinine levels were significantly associated with higher levels of TARC $(p=.002)$ but not BAFF $(p=.186)$. Glycoprotein130 (gp130) levels were inversely associated with smoking, but associations were not significant after multiple comparison adjustment. An expression quantitative trait locus (eQTL) analysis evaluating the effect on biomarker levels of DNA methylation across all loci and loci in the biomarker genes is being conducted and will be presented. Conclusions: TARC and BAFF, both associated with Th2 immunity, were increased in smokers, accounting for genetic factors.

\section{THE STUDY OF THE RELATIONSHIP BETWEEN HUMAN GUT MICROBIOTA AND BMI FOR JAPANESE TWINS}

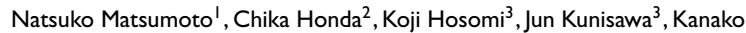
Akada $^{2}$, Mikio Watanabe ${ }^{1,2}$, Yoshinori Iwatani ${ }^{1,2}$, Osaka Twin Research Group ${ }^{2}$, and Rie Tomizawa ${ }^{2,4}$

${ }^{I}$ Department of Biomedical Informatics, Division of Health Sciences, Osaka University Graduate School of Medicine, Osaka, Japan

${ }^{2}$ Center for Twin Research, Osaka University graduate school of medicine, Osaka, Japan

${ }^{3}$ Laboratory of Vaccine Materials and Laboratory of Gut Environmental System, National Institutes of Biomedical Innovation, Health and Nutrition, Osaka, Japan ${ }^{4}$ Department of Biodesign for Healthcare Innovation, Osaka University Graduate School of Medicine, Japan

Introduction: Obesity is considered to be a leading cause of various lifestyle-related diseases, such as diabetes, high blood pressure, and cardiovascular diseases. Thus, prevention of obesity is a very important target in extending healthy life expectancy. Recently it is suggested that the human gut microbiota correlate with obesity, and many researches are conducted to prevent obesity through intervention of gut microbiota. It was reported that the proportion of Firmicutes was increased and that of Bacteroidetes was decreased in the obese group compared to non-obese group. However not all data from previous studies correspond to this report, we have no hard evidence about it. It is necessary to identify which bacteria is related to being non-obese as well as obese to better understand the relationship between human gut microbiota and obesity. Japanese are known to be low on BMI and live long. They keep comparatively traditional dietary habits including diverse and balanced meals. In addition, it was reported that the Japanese have unique gut microbiota compositions compared to other countries. Ours is the very first study focusing on the relationship between human gut microbiota and health using the samples from Japanese twins. We aim to indicate how the genetic and environmental factors affect Japanese gut microbiota 
composition. Materials and Methods: We collected fecal samples of Japanese male twin pairs who are registered in Center for Twin Research, Osaka University Graduate School of Medicine. Then we extracted bacterial DNA and identified bacteria in each human gut microbiota by $16 \mathrm{~S}$ rRNA analysis using the next generation sequencer (NGS). We analyzed 48 samples, and 46 of them were complete twin pair samples (23 pairs). We focused on the two monozygotic twin pairs who had low BMI $(\mathrm{BMI}<20)$, and compared the compositions of gut microbiota. Result: One twin pair showed relatively similar compositions of gut microbiota (Firmicutes: $59.1 \%$ and $58.3 \%$, Bacteroidetes: $35.8 \%$ and $30.9 \%$ ), and both individuals had no diseases. On the other hand, the other pair showed quite different (Firmicutes: $23.8 \%$ and $38.1 \%$, Bacteroidetes: $8.2 \%$ and $50.3 \%$ ). In this pair, one individual reported diabetes and the other had no reported diseases. Conclusion: Even among monozygotic twins with low BMI, they showed concordant and discordant compositions of their gut microbiota.

\section{DIFFERENCES IN FECAL MICROBIOTA IN LONG-TERM HODGKIN LYMPHOMA SURVIVORS AND THEIR UNAFFECTED TWINS.}

Wendy Cozen', Nancy Huang ${ }^{2}$, Venu Lagishetty ${ }^{2}$, Marta Epeldegui ${ }^{2}$, Yang Yu', James Buxbaum ', Joshua Millstein', Bharat N. Nathwani ${ }^{3}$, Amie E. Hwang', Guoqin $\mathrm{Yu}^{4}$, Thomas M. Mack', Otoniel Martinez-Maza ${ }^{2}$, and Jonathan P.jacobs ${ }^{2}$ ${ }^{I}$ Keck School of Medicine of USC, University of Southern California, Los Angeles, California, USA

${ }^{2}$ David Geffen School of Medicine, University of California at Los Angeles (UCLA), Los Angeles, California, USA

${ }^{3}$ City of Hope National Medical Center, Duarte, California, USA

${ }^{4}$ National Cancer Institute, National Institutes of Health, Bethesda, Maryland, USA

Introduction: Immune dysregulation is clinically and pathologically associated with adolescent /young adult Hodgkin lymphoma (AYAHL) at diagnosis and alterations in inflammatory cytokine levels are evident years after cure. Gut bacteria play a role in modulating immune responses. In a pilot study, AYAHL survivors had significantly lower fecal microbial diversity compared to their unaffected twin. We conducted a follow-up study to determine whether there are differences in the relative abundance of specific fecal microbiota in survivors compared to their unaffected twins. Materials and Methods: The subjects are twins enrolled in the USC International Twin Study. One twin was diagnosed with histologically verified AYAHL $<50$ years old, at least 10 years ago (mean interval since diagnosis $=22$ years ago), and the other twin has remained unaffected. 24 AYAHL survivors and their unaffected twins provided fresh stool samples, frozen at -80 , shipped to our laboratory and maintained at that temperature until use. DNA extraction was performed using bead beating with the MO BIO Powersoil kit. Sequencing of the $16 \mathrm{~S}$ ribosomal DNA V4 region was performed using an Illumina HiSeq 2500. Raw sequence data was processed in QIIME and 97\% OTUs picked using the Silva database. Alpha diversity was compared with a paired t-test. Relative abundance of genera were determined using DESeq2, based upon negative binomial models with twin pair as a covariate. P-values were adjusted for multiple comparisons. Results: Sequencing yielded an average of 219,373 reads, with a minimum of 64,742 and a maximum of 391,120. Among like-sex twins, survivors had lower alpha diversity compared to their unaffected twins $(p=.04)$. There were no differences in beta-diversity. Significant differences in relative abundance of the following genera were observed between AYAHL survivors and their unaffected twins: Streptococcus $(p=9.7 \times 10-5)$, Sellimonas $(p=.005)$, Candidatus Stoquefichus $(p=.020)$, unclassified S24-7 ( $p=.035)$, Faeclitalea $(p=.035)$, Veillonella $(p$ $=.040)$, Faecalibacterium $(p=.026)$ and [Eubacterium] oxidoreducens group $(p=.028)$. The last two taxa were more abundant in survivors compared with unaffected twins and the remaining taxa were more abundant in unaffected twins compared with survivors. Conclusions: Even after decades, AYA HL survivors harbor fecal microbiota that differ from that of their unaffected twins who never experienced the lymphoma or its treatment.

\section{KEY INDICATORS IN MULTIPLE BIRTHS CARE IN THE UNITED KINGDOM AND THEIR RELATIONSHIP WITH STILLBIRTH AND NEONATAL DEATHS}

Evangelia E. Antoniou', Keith Reed ${ }^{2}$, Amy McCarthy ${ }^{2}$, Helen Turier ${ }^{2}$, and Nikki Roberts ${ }^{2}$

${ }^{1}$ University of Maryland University College Europe, Germany

${ }^{2}$ The Twins and Multiple Births Association, United Kingdom

Introduction: According to the 2015 MBRRACE report the rate of perinatal mortality in the UK has decreased from 6.04 to 5.61 deaths per 1,000 total births over the period 2013 to 2015. However, there is a small decrease in the rate of neonatal mortality. This report provides information on perinatal deaths and indicators related to mortality rates by Neonatal Networks across England. Materials and Methods: National data were provided by the Office of National Statistics, the National Neonatal Research Database, the NHS National Reporting and Learning System and the TAMBA maternity survey. This report explored: time trends in rates of stillbirth and neonatal deaths in multiple pregnancies compared to singleton pregnancies; rates of admissions to neonatal care and recorded patient safety incidents; NICE compliance and its influence on stillbirths and neonatal admissions; the influence of patient care on satisfaction rates; the influence of the number of multiple pregnancies on perinatal mortality rates; the estimated number of admissions to neonatal care if networks had the lowest admission rate; the number of baby lives who would be saved if networks had the lowest neonatal and stillbirth rates. The main findings are presented in time trend graphs and tables. For comparison purposes the mortality rates for individual networks are presented compared to the UK averages. Results: The multiple births across UK regions demonstrate higher perinatal mortality rates in comparison to singleton pregnancies; admission to neonatal care is related with patient safety incidents; greater levels of compliance with antenatal guidelines relate to lower rates of stillbirths but don't influence neonatal admissions; patient care is related to positive patient satisfaction rates; smaller cohorts of multiple pregnancies may result in poorer care, patient satisfaction and outcomes; admissions to neonatal care would be reduced by 3,067 per year if all neonatal networks had the lowest admission rate; 420 baby lives would be saved every year if all neonatal networks had the lowest perinatal mortality rates. Conclusions: A better understanding of the causes of preterm birth and the development of interventions to prevent preterm birth are needed for a sustained reduction in the rates of perinatal deaths and in particular neonatal deaths. Standardised guidance for intrapartum care in multiple pregnancies could reduce stillbirths, neonatal deaths and the wide differences in neonatal admissions.

\section{RELATIONSHIP OF LUMBAR DISC PROTRUSIONS WITH MTDNA COPY NUMBER AND TELOMERE LENGTH IN TWINS}

Dávid László Tárnoki', Adam Domonkos Tárnoki', Dóra Melicher², Anett Illés ${ }^{3}$, András Falus ${ }^{2}$, Mária Judit Molnár ${ }^{3}$, Marcell Szily', Dániel Tamás Kovács', Bianka Forgó ', László Kostyál ${ }^{4}$, and Csaba Oláh ${ }^{4}$

${ }^{1}$ Semmelweis University, Department of Radiology, Budapest, Hungary

${ }^{2}$ Semmelweis University, Department of Genetics, Cell- and Immunobiology, Budapest, Hungary

${ }^{3}$ Semmelweis University, Institute of Genomic Medicine and Rare Disorders, Budapest, Hungary

${ }^{4}$ Borsod-Abaúj-Zemplén County and University Teaching Hospital, Department of Neurosurgery, Miskolc, Hungary

Introduction: There is evidence that telomere length (TL) and age are related to the presence of degenerative disc disease (DDD), a common condition characterized by progressive loss of human nucleus pulposus cells and extracellular matrix. Although there is a 
slight genetic component in DDD, the role of mtDNA copy number (mtDNAcn) and TL in the development of degenerative disc disease has never been assessed in twins. Materials and Methods: 92 twins from the Hungarian Twin Registry (36 monozygotic, and 10 dizygotic twin pairs, mean age $53 \pm 15$ years) underwent lumbar spine MRI (Siemens Magnetom Verio 1.5T) and phlebotomy. After DNA extraction from peripheral blood mononuclear cells, absolute telomere length (kilobase per diploid cell) and absolute mitochondrial copy number (number of circular DNA per cell) were analysed by qPCR standard curve method. The presence and number of disc protrusions and herniation was recorded and compared with mtDNA copy number and telomere length. Results: In twins with presence of disc protrusion $(n=40)$, mtDNAcn was insignificantly higher than in twins without disc protrusion $(n=20)(189 \pm 61$ vs. 160 $\pm 67, p=.105)$. TL was shorter in twins with disc protrusion (189 \pm 87 vs. $236 \pm 106, p=.09$ ), especially in MZ twins ( $p=.06$ for both parameters). In 5 monozygotic pairs discordant for the presence of disc protrusion, mtDNAcn and TL was insignificantly less and shorter (mean $\triangle$ mtDNAcn difference $-11.1, p=.783$ and mean $\Delta \mathrm{TL}-14.1, p=.860)$ in the affected monozygotic twin. Conclusions: Albeit our population was small, it seems that the presence of lumbar disc protrusion might be related to higher mtDNAcn and shorter TL. Further research is needed to examine common genetic factors in the background.

\section{SERIAL CERVICAL CONSISTENCY INDEX MEASUREMENTS AND PREDICTION OF PRETERM BIRTH $<34$ WEEKS IN TWIN PREGNANCIES}

Hadar Rosen, Vasi Stratulat, Nimr Melamed, Amir Aviram, Rania Okby, Jon Barrett, and Phyllis Glanc

Maternal Fetal Medicine, Department of OB/GYN, Sunnybrook Health Sciences Centre, University of Toronto, Toronto, Canada

Objective: To assess the effectiveness of serial Cervical Consistency Index (CCI) measurements in the predictions of spontaneous preterm birth $(\mathrm{sPTB})<34$ weeks in twin pregnancies. Study Design: Prospective observational cohort study in a single tertiary center including women with twin pregnancies. CCI was calculated as the ratio of maximum anteroposterior diameter of the cervix measurements at maximal compression and at rest, and was measured (as was standard cervical length (CL)) via transvaginal ultrasound exam at 2 week intervals between 16-30 weeks gestation. Analysis was made in clusters according to gestational age: 16-19 weeks, 20-23 weeks and 24-29 weeks. Primary outcome was preterm birth prior to $34+0$ weeks of gestation. Results: A total of 76 parturients with twin gestations were included in the study, out of which 17 (22.4\%) delivered at 0.99) (Figure). Conclusion: Serial CCI measurements in twin pregnancies were not proven useful predicting spontaneous preterm birth prior to 34 weeks gestational age.

\section{SERIAL UTERO-CERVICAL ANGLE MEASUREMENTS AND PRETERM BIRTH $<34$ WEEKS IN TWIN PREGNANCIES}

Hadar Rosen, Vasi Stratulat, Nir Melamed, Amir Aviram, Rania Okby, Jon Barrett, and Phyllis Glan

Maternal Fetal Medicine, Department of OB/GYN, Sunnybrook Health Sciences Centre, University of Toronto, Toronto, Canada

Objective: To assess the use of serial utero-cervical angle (UCA) measurements to predict spontaneous preterm birth (sPTB) $<34$ weeks in twin pregnancies. Study Design: Prospective observational cohort study in a single tertiary center including women with twin pregnancies. UCA was defined as the angle between the lower uterine segment and the cervical canal. UCA, as well as standard cervical length (CL) were measured via transvaginal ultrasound at 2 week intervals between 16-30 weeks gestation. Analysis was made in clusters according to gestational age: 16-19 weeks, 20-23 weeks and 24-29 weeks. Primary outcome was preterm birth prior to $34+0$ weeks of gestation. Results: Overall, 76 women with twin gestations were eligible for analysis, of whom $17(22.4 \%)$ delivered spontaneously prior to 34 completed weeks of gestation. For women who delivered prior to 34 weeks of gestation, mean UCA measurement (expressed in degrees) at 16-19, 20-23 and 24-29 weeks' gestation was $105.6 \pm 27.9,114.4 \pm 22.5$ and $100.2 \pm 28.6$, respectively, and for women who delivered at 34 weeks' gestation or later $110.6 \pm 19.3$, $113.0 \pm 19.6$ and $116.6 \pm 15.9$, respectively. The differences between UCA measurements at 24-29 weeks proved to be significantly lower among women who delivered prior to 34 weeks' gestation $(p<$ .035). Conclusion: While serial measurements of UCA up to 23 weeks of gestation did not predict spontaneous preterm birth among twins, a single measurement at 24-29 weeks of gestational age may assist in the prediction of it.

\section{SINGLE MID-TRIMESTER UTERO-CERVICAL ANGLE MEASUREMENT AND PREDICTION OF PRETERM BIRTH IN TWIN PREGNANCIES}

Hadar Rosen, Vasi Stratulat, Nir Melamed, Amir Aviram, Rania Okby, Jon Barrett, and Phyllis Glanc

Maternal Fetal Medicine, Department of OB/GYN, Sunnybrook Health Sciences Centre, University of Toronto, Toronto, Canada

Objective: To assess the use of mid-trimester utero-cervical angle (UCA) to predict spontaneous preterm birth in twin pregnancies. Study Design: Prospective observational cohort study in a single tertiary center including women with twin pregnancies. UCA was defined as the angle between the lower uterine segment and the cervical canal. UCA, as well as standard cervical length (CL) were measured via transvaginal ultrasound exam at $18+0-23+0$ weeks' gestation. The primary and secondary outcomes were defined as spontaneous preterm birth prior to $34+0$ weeks, and prior to $37+0$ weeks, respectively. Results: Overall, 76 women with twin gestations were eligible for analysis, of whom 17 (22.4\%) delivered spontaneously prior to 34 completed weeks of gestation, and 42 (55.3\%) delivered spontaneously prior to 37 completed weeks of gestation. Regardless of gestational age cut-off chosen (34 or 37 weeks), neither CL nor UCA were significantly different between those delivering before or after the cut-off. For 34 weeks' gestation, the area-under-the-curve (AUC) of CL was 0.652 and of UCA 0.555 . CL of $3.95 \mathrm{~cm}$ and UCA of 114 degrees reached the best test performance with sensitivities of $57 \%$ and $44.8 \%$ and specificities of $68.7 \%$ for both, respectively. Conclusion: Single mid-trimester UCA measurement in twin pregnancies does not seem the predict spontaneous preterm birth prior to 34 or 37 weeks of gestation.

\section{PREDICTION OF PRETERM BIRTH IN TWIN PREGNANCIES BY SINGLE MID-TRIMESTER CERVICAL CONSISTENCY INDEX MEASUREMENT}

Hadar Rosen, Vasi Stratulat, Nir Melamed, Amir Aviram, Rania Okby, Jon Barrett, and Phyllis Glanc

Maternal Fetal Medicine, Department of OB/GYN, Sunnybrook Health Sciences Centre, University of Toronto, Toronto, Canada

Objective: To assess the utility of a single, mid-trimester Cervical Consistency Index (CCI) measurement as a prediction tool for spontaneous preterm birth (sPTB) in unselected twin pregnancies. Study Design: Prospective observational cohort study in a single tertiary center including women with twin pregnancies. CCI was calculated as the ratio of maximum anteroposterior diameter of the cervix measurements at maximal compression and at rest, and was measured (as was standard cervical length (CL)) via transvaginal ultrasound exam at $18+0-23+0$ weeks' gestation. sPTB $<34$ weeks and SPTB $<37$ weeks were defined as primary and secondary outcomes, respectively. Results: A total of 76 parturients with twin gestations were included in the study, out of which $17(22.4 \%)$ delivered spontaneously at $<34$ weeks of gestation, and $42(55.3 \%)$ at $<37$ weeks 
of gestation. CCI was not different between those delivering prior to 34 or 37 weeks of gestation, and those who delivered after these gestational ages (Table). The area-under-the-curve (AUC) of CL was 0.645 and of UCA 0.538 , for delivery $<34$ weeks' gestation. CL of $3.95 \mathrm{~cm}$ and CCI of 71.05 had the best test performance with sensitivities of $57 \%$ and $34.5 \%$ and specificities of $64.5 \%$ and $88.2 \%$, respectively. Conclusion: Single mid-trimester CCI measurement does not seem to play a pivotal role in the prediction of spontaneous preterm birth in twin gestations.

\section{VANISHED TWIN: OBSTETRIC AND PERINATAL OUTCOMES AND ASSOCIATION WITH METHYLENETETRAHYDROFOLATE REDUCTASE (MTHFR) POLYMORPHISMS(S)}

Murat Aykut Ozek', Ergun Karaagaoglu² , Gokcen Orgul $^{3}$, Fatma Gumruk ${ }^{4}$, Murat Yurdakok ${ }^{5}$, and M. Sinan Beksac ${ }^{3}$

${ }^{1}$ Perinatology Department, Cengiz Gokcek Womens Health Hospital, Gaziantep, Turkey ${ }^{2}$ Department of Biostatistics, Hacettepe University, Ankara, Turkey

${ }^{3}$ Division of Perinatal Medicine, Department of Obstetrics and Gynecology, Hacettepe University, Ankara, Turkey

${ }^{4}$ Division of Hematology, Department of Pediatrics, Hacettepe University, Ankara, Turkey

${ }^{5}$ Division of Neonatology, Department of Pediatrics, Hacettepe University, Ankara, Turkey

Introduction: The vanished twin (VT) phenomenon is defined by the disappearance of an entire gestational sac or one of the fetuses after detection of cardiac activity during multiple pregnancies. The pathophysiology behind VT is thought to be the same as that of miscarriages, which are mainly due to genetic disorders that lead to formation of blighted ovum and early pregnancy loss. A relationship between methylation pathway disorders and poor pregnancy outcomes (repeated miscarriages, intrauterine growth retardation, preterm delivery, preeclampsia, and ablation placenta), as well as chromosomal abnormalities and congenital malformations, has also been reported. Our research aimed to investigate the outcomes of pregnancies with vanished twin and its possible association with methylenetetrahydrofolate reductase (MTHFR) polymorphisms. Material and Methods: In this study, we evaluated the outcomes of 'VT gestations' and compared them with those of singleton pregnancies, spontaneous twins, and twins concieved through assisted reproductive techniques (ART). We also investigated the possible relationship between the VT phenomenon and maternal polymorphisms of MTHFR genes. This study consisted of 30 of 38 VT pregnancies delivered at our hospital (group 1: VT group), 109 singletons (group 2), 70 spontaneous twins (group 3), and 101 in vitro fertilization (IVF) / intracytoplasmic sperm injection (ICSI) twins (group 4).

Results: Most patients in group 1 (28/30) were tested for MTHFR genes (C677T or A1298C polymorphisms). Eight of the 38 pregnancies with VT $(21.1 \%)$ resulted in a miscarriage. The prevalence of ' 2 or more pregnancy losses' in the 'obstetric history' in group 1 was higher $(23.3 \%)$ than those in the other groups $\left(p=0.007, \chi^{2}\right.$ $=17.8$ ). The allelic frequencies of MTHFR 677 and MTHFR 1298 in group 1 were 0.268 and 0.429 , respectively (higher than those in healthy population). The median birthweights in groups $1,2,3$, and 4 were $2,940,3,200,2,300$, and 2,095 g respectively. The prevalence of respiratory distress syndrome was significantly higher in the IVF/ICSI twin pregnancy group $\left(\mathrm{p}<.001, \chi^{2}=21.2\right)$. Conclusions: This is the first study evaluating the 'vanished twin' phenomenon in terms of MTHFR polymorphisms, to the best of our knowledge. Multiple pathophysiological processes are thought to be responsible for VT phenomenon. The coincidence of VT and MTHFR polymorphisms might play an incidental or factual role in this connection.

\section{TWIN REVERSED ARTERIAL PERFUSION SYNDROME: A CASE REPORT OF ACARDIAC FETUS IN MONOCHORIAL-DIAMNIOTIC TWIN PREGNANCY}

João Pinto, Vera Ribeiro, Angela Ferreira, Vera Mourinha, and Diana Almeida Prenatal Department, University Hospital of Algarve, Faro, Portugal

Introduction: Multiple gestations involve a high-risk situation for both mother and fetuses, but monozygous twin pregnancy is the ultimate challenge for an obstetrician. Serious complications may occur during the pregnancy. One of these complications is reversed arterial perfusion (TRAP). We present a case of a very rare TRAP-acardiac twin pregnancy which is reported to happen in $1 \%$ of monochorionic twin pregnancy and 1 in 35,000 of all pregnancies. Case Report: A 37-year-old pregnant woman, in her sixth pregnancy presented to us at 12 weeks and 6 days of a monochorionic twin pregnancy with a malformed twin and a normal one. Ultrasonography evaluation revealed a monozygotic twin pregnancy with a first normal fetus (pump twin) and a second acardiac fetus (the head, the neck, the trunk and upper limbs were also absent). A thin complete membrane was present between the two twins. The patient was counseled about the risks of this pregnancy according the most recent literature: risk of chromosomal abnormalities, involution of the acardiac twin, hemodynamic complications which could lead to the involution of the pregnancy, premature birth and polyhydramnios as well as possible interventions and its risks. She was also informed about the possibility of legal termination of pregnancy, however as the chorionic villus sampling had a normal feminine karyotype, she decided to keep it. The women was periodically monitored by performing serial ultrasounds. In addition, at 17 weeks and 3 days of pregnancy, modifications were observed. The second twin had no blood flow while the first twin was with normal range of growth. A second trimester morphology ultrasound performed at 20 weeks described a normal first twin and second twin measuring $20 \mathrm{~mm}$ with no blood flow, anasarca and no amniotic fluid. Conclusion: Diagnosis of acardiac twin can be made the first trimester by ultrasound and doppler. Early sonographic prenatal diagnosis improves the survival rate of the normal twin. In literature conservative treatment is best suited for salvation of the pump twin when the acardiac twin is less than one fourth the weight of the pump twin. Treatment at appropriate time improves the survival of the pump twin by $95 \%$. Invasive intervention is justified when the acardiac twin receive more than $70 \%$ of the pump twin; however, the chance of survival is still low. The comprehension and study of these cases is extremely important in order to offer maximum survival and health chances to the viable fetus.

\section{THE MOSCOW LONGITUDINAL STUDY OF NEWBORN TWINS: PILOT STUDY FINDINGS OF IVF AND NON-IVF GROUP}

Veronika Odintsova ${ }^{1,2}$, Alexander Konoplyannikov $^{3}$, Lali Sichinava ${ }^{3}$, Oleg Latyshkevich $^{4}$, Elena Nurahova ${ }^{4}$, Irina Voronina ${ }^{2}$, Izabella Airapetian ${ }^{2}$, and Sergey Malykh ${ }^{2}$

${ }^{1}$ Research Center for Emergency Medicine, Moscow Healthcare Department, Moscow, Russia

${ }_{2}^{2}$ Psychological Institute of Russian Academy of Education, Moscow, Russia

${ }^{3}$ Russian National Research Medical University, Moscow, Russia

${ }^{4}$ Center for Family Planning and Reproduction, Moscow, Russia

Introduction: The longitudinal studies are crucial for the current psychological, epidemiological and clinical research. The cohorts of families, enrolled before or early after the birth of the child, comprise a significant part of empirical data in each of those research areas. The results of existing longitudinal studies suggest that more research is needed for the understanding of the role of child development and its influence on human lifespan. Despite the importance of the information on child development, provided by these longitudinal studies, the necessity of the studying of family cohorts at the 
period of pregnancy is becoming more obvious. Such family cohorts will provide a way to assess how the influence of different environments embeds itself into the child's biology, and further influence the developmental trajectory. Materials and Methods: All families are recruited through family planning clinics during pregnancy. Two cohorts are assessed: twins born through IVF (40 families) and naturally conceived twins (40 families). Data are collected at five time points: 1 st and 2nd measures were conducted during pregnancy, $3 \mathrm{rd}$ - at birth, 4th and 5th will be done during the 1st year after birth. Physical, cognitive and socio-emotional development will be assessed using a specially developed battery which includes valid and currently widely used questionnaires and tests. The biomaterials are collected: cord blood for molecular genetic and epigenetic analysis; mothers' hair samples for biochemical analysis. Results: For today, our research team has developed a protocol, organized recruitment of 80 participants and data collection in a hospital with the support of medical staff. Data collection on the first three points has been done and interaction with laboratories analyzing bio-samples has been organized. A comparative analysis of standard pre- and postnatal data according to the medical records of twins born with IVF and naturally conceived twins was carried out. The relationship of a number of behavioral characteristics of the mother (before and during pregnancy) with the physical characteristics of twins was analyzed. The research will be continued at the 4th and 5th points. Conclusions: The results of this longitudinal genetically informative study will contribute to the understanding the underpinnings of mental development, including gene-environment interaction in the development of individual differences in social, emotional and cognitive development.

\section{FETAL GROWTH DISCORDANCY IN MULTIPLES}

Lali Sichinava', Olga Panina ${ }^{2}$, and Ketevan Gamsakhurdiya ${ }^{3}$

${ }^{1}$ Moscow National Research Medical University, Moscow, Russia

${ }^{2}$ Lomonosov Moscow State University, Moscow, Russia

${ }^{3}$ Center Family Planning And Reproduction, Moscow, Russia

Introduction: In addition to perinatal mortality and morbidity attributable to preterm delivery fetuses in multiple gestations are vulnerable to a variety of complications. The significant weight differences between twins may have different causes. Twin birth weight difference is an important factor for poor perinatal outcome. The degree of birth weight discordance that might be of great clinical importance in management of twin pregnancies is not yet established. The aim of this study was to ascertain the relationship between the degree of birth weight discordance and pregnancy outcome in dichorionic and monochorionic twins. Materials and Methods: A retrospective study of 120 twin pregnancy births of known chorionicity (65 DC and $55 \mathrm{MC}$ ) was performed. The following degrees of fetal discordancy were analyzed: $<15 \%, 15-20 \%, 20-25 \%,>25 \%$. According to these degrees, birth weight discordance was found in $64,15,12$, and 29 pregnancies. Adverse perinatal outcomes included preterm delivery (PD) rate ( $\leq 34$ weeks), IUGR, fetal distress, low Apgar score (less than 7), perinatal death, pathological neurosonographic findings. Results: Birth weight discordance was significantly more prevalent in MC twins in the group of patients with weight difference more than $25 \%$ : 32.4 versus $19.4 \%$ in DC twins. In the other groups of patients (less than 25\%) chorionicity itself did not significantly influenced the frequency of fetal weight discordance. Birth weight discordance $>20 \%$ in MC twins was associated with the prematurity of $21.6 \%$ versus $11.1 \%$ in discordancy $\leq 20 \%$, in DC twins $-21.7 \%$ versus $17.5 \%$ accordingly. In MC twins IUGR was diagnosed in $11.1 \%$ of twins with discordance $\leq 20 \%$, in $42.9 \%$ in discordancy $>20 \%$; in DC $-6.9 \%$ and $28.6 \%$ accordingly. Fetal distress in IUGR MC group was six times higher comparing with the patients without IUGR (43.3\% vs. $6.3 \%)$. The highest rate of low Apgar score - 33.3\% and even more in patients with severe IUGR was in the MC group with discordancy $>25 \%$. Patholog- ical neurosonographic findings were $33,3 \%$ and $46,3 \%$ in MC twins with discordancy $\leq 20 \%$ and $>20 \%$ accordingly. Conclusions: Fetal weight discordance $(>20 \%)$ is associated with the high risk of adverse perinatal outcomes. Ultrasonographic monitoring of growth within a twin pair is a mainstay in the management of twin gestation, especially in monochorionic twins. Depending on the degree of discordance, the gestational age and chorionicity fetal surveillance may be indicated, especially in IUGR fetus.

\section{THE IMPACT OF THE IN UTERO ENVIRONMENT ON DNA METHYLATION AND BRAIN DEVELOPMENT: LONGITUDINAL INVESTIGATIONS IN MONOZYGOTIC TWINS}

Linda Booij ${ }^{1,2}$, Kevin F. Casey ${ }^{2}$, Tiago R. Magalhaes ${ }^{3}$, Elmira Ismaylova ${ }^{2}$, Melissa L. Levesque $^{2}$, Frank Vitaro ${ }^{2,4}$, Mara Brendgen ${ }^{2,5}$, Ginette Dionne ${ }^{6}$, Moshe Szyf ${ }^{7}$, Michel Boivin ${ }^{6}$, and Richard E. Tremblay $2,8,9$

${ }^{1}$ Psychology, Concordia University, Montreal, Canada

${ }^{2}$ CHU Sainte-Justine, Montreal, Canada

${ }^{3}$ Academic Centre on Rare Diseases, University College Dublin, Dublin, Ireland

${ }^{4}$ Psychoeducation, University of Montreal, Montreal, Canada

${ }^{5}$ Psychology, UQAM, Montreal, Canada

${ }^{6}$ Psychology, University of Laval, Montreal, Canada

${ }^{7}$ Department of Pharmacology and Therapeutics, McGill University, Montreal, Canada ${ }^{8}$ Psychology \& Pediatrics, University of Montreal, Montreal, Canada

${ }^{9}$ School of Public Health, Physiotherapy and Sports Science, University College Dublin, Dublin, Ireland

Background: Studies have shown that the quality of the in utero environment, which can be indexed by birth weight (BW), is predictive of brain development in adolescence and adulthood. Work in monozygotic (MZ) twins suggests that this association is driven by non-shared environmental factors; and that such association could be the result of in utero impacts on DNA methylation. In this presentation, we present data on the association between the prenatal environment (as indexed by BW), DNA methylation and brain processes in MZ twins. Materials and Methods: 52 adolescent and 12 newborn MZ twin pairs with various levels of BW discordance were tested. DNA methylation was obtained from mouth swabs at 1 month (newborns) and at age 15 years (adolescents) and assessed with the Illumina Infinium HumanMethylation450 BeadChip Kit. In addition to measures of behavior and family environment collected in both twin samples, adolescent twins underwent an fMRI at age 15. Results: In adolescent MZ twins, BW discordance was associated with discordance in cortical morphology. Genes involved in neurodevelopment were tentatively identified as mediators of the BW-cortical volume and BW-cortical surface area relationships. We also found in the adolescent twins that discordance in DNA methylation in the serotonin transporter (SLC6A4) gene, a gene important for emotion regulation, was linked to discordance in frontal-limbic neural responses to negative stimuli. Preliminary analyses of the newborn twin data showed that intrapair methylation variation was lower than in adolescent twins, but (as in adolescents) organized in certain functional gene pathways. Intriguingly, 23 functionally relevant probes were differentially methylated as a function of BW discordance in both the adolescent and newborn twins. We also found a very preliminary positive trend between BW discordance and DNA methylation discordance in serotonin system genes (SLC6A4, MAOA) in the newborn twins. Conclusions: The association between BW and cortical morphology in adolescence appears to be attributable to in utero environmental effects, and DNA methylation may play a role in mediating this relationship. Future epigenetic-imaging twin studies in young twins are needed to study whether impacts of in utero environment on early development are similar to its impacts in adolescence. Such research will inform the design and optimum timing of early preventive interventions to reduce risk for health problems. 


\section{ASSOCIATION BETWEEN SMOKING AND DEPRESSION: A LONGITUDINAL INVESTIGATION AMONG TWINS FROM LATE ADOLESCENCE TO YOUNG ADULTHOOD}

Anu Ranjit ${ }^{1}$, Tellervo Korhonen ${ }^{1,2,3}$, Jadwiga Buchwald ${ }^{1,2}$, Kauko Heikkila $^{1,2}$, Annamari Tuulio-Henriksson ${ }^{4,5}$, Richard J. Rose ${ }^{6}$, Jaakko Kaprio ${ }^{1,2}$, and Antti Latvala ${ }^{1,2}$

${ }^{I}$ Department of Public Health, University of Helsinki, Helsinki, Finland

${ }^{2}$ Institute for Molecular Medicine FIMM, University of Helsinki, Helsinki, Finland ${ }^{3}$ Institute of Public Health and Clinical Nutrition, University of Eastern Finland, Kuopio, Finland

${ }^{4}$ Research Unit, Social Insurance Institution, Helsinki, Finland

${ }^{5}$ Department of Psychology and Logopedics, University of Helsinki, Helsinki, Finland ${ }^{6}$ Department of Psychological and Brain Sciences, Indiana University, Bloomington, United States

Introduction: Longitudinal research is needed to understand the development of the relationship between cigarette smoking and depressive symptoms. Our aim was to examine both the association of adolescent cigarette smoking with depressive symptoms in young adulthood and the reverse association between adolescent depression and subsequent smoking. Materials and Methods: We analyzed prospective longitudinal data from twins participating in the late adolescent (mean age 17.5) and young adult (mean age 22) surveys of the FinnTwin12 study $(n=2921)$. In these two surveys, both smoking patterns and depressive symptoms were assessed. Depressive symptoms were assessed with the 10-item version of the General Behaviour Inventory (GBI). We used negative binomial regression and multinomial logistic regression analyses, which were further adjusted for multiple confounders. Furthermore, we conducted within pair analyses to control for familial confounding. Results: Cigarette smoking during adolescence predicted depressive symptoms in young adulthood. The incidence rate ratio (IRR) estimates, when adjusted for all confounders including baseline depressive symptoms, were higher among those who had smoked $>100$ cigarettes (IRR $=1.15,95 \%$ CI $[1.02 .1 .30]$ ), and among daily smokers $($ IRR $=1.17,95 \%$ CI 1.02- 1.34$)$ compared to never smokers. In the within-pair analysis, compared to the individual analysis, the association was slightly reduced in dizygotic pairs (DZ) $($ IRR $=1.11,95 \%$ CI $[0.93,1.34])$, and fully attenuated in monozygotic pairs $(\mathrm{MZ})(\mathrm{IRR}=0.80,95 \% \mathrm{CI}[0.62,1.03])$ for those who had smoked $>100$ cigarettes. Among daily smokers, a similar pattern was observed: $\mathrm{DZ}(\mathrm{IRR}=1.06,95 \% \mathrm{CI}[0.87,1.28])$ and $\mathrm{MZ}$ $(\mathrm{IRR}=0.86,95 \% \mathrm{CI}[0.67,1.11])$. For the reverse association, relative risk ratio (RRR) estimates for becoming a daily smoker were slightly but statistically significantly elevated (RRR $1.09,95 \%$ CI $[1.00,1.18])$ for those with higher depression scores in late adolescence (compared to remaining never smokers), whereas the depression score did not predict subsequent occasional smoking. Conclusions: Cigarette smoking during adolescence is a significant predictor of depressive symptoms later in life. Additionally, depressive symptoms during adolescence may slightly increase the likelihood of becoming a daily smoker later. However, the associations may not be independent of several measured confounding factors as well as shared genetic and other familial influences.

\section{HOUSEHOLD STRESS INCREASES THE HERITABILITY OF EMOTIONAL EATING IN CHILDHOOD: A GENE-ENVIRONMENT INTERACTION STUDY}

\section{Moritz Herle', Alison Fildes ${ }^{2}$, Fruhling Rijsdijk ${ }^{3}$, and Clare Llewellyn'}

${ }^{I}$ Department of Behavioural Science and Health, University College London, London, United Kingdom

${ }^{2}$ School of Psychology University of Leeds, Leeds, United Kingdom

${ }^{3}$ Social, Genetic and Developmental Psychiatry Centre, King's College London

London, United Kingdom

Introduction: Emotional overeating (EOE) and undereating (EUE), the tendency to eat more or less in response to stress, have been linked with greater risk for obesity and mental health problems. Twin research in children has indicated that variation in EOE and
EUE largely stems from shared environmental factors, while heritability is low. However, the diathesis-stress model hypothesises that genetic effects increase in adverse environments, and emotional eating is a behavioral response to environmental stressors. This study aims to investigate whether genetic influence on EOE and EUE is conditional on household stress levels. Materials and Methods: Participants were 907 five-year-old twin pairs from the Gemini cohort. EOE and EUE were measured using the Child Eating Behaviour Questionnaire. Household stress was assessed using the Confusion, Hubbub and Order Scale. Two continuous moderator twin models were conducted to test if increasing household stress changes the etiology of child EOE and EUE. All analyses controlled for age at measurement, sex and gestational age of the twins. Results: Findings suggested significant gene-environment interactions underlying EOE and EUE. Variance explained by additive genetic factors rose significantly with increasing stress in the home. Overall effect of the interaction was small, but variance explained by genetic effects at highest level of household stress was four times larger than in the absence of any household stress. However, shared environmental factors explained the majority of variation in both EOE and EUE at all levels of household stress. For EUE, variance explained by non-shared environmental factors decreased slightly with increasing home stress. Conclusions: Results position the development of child EOE and EUE within the diathesis-stress framework, whereby exposure to increased household stress results in greater genetic influence. However, shared-environmental factors within one family remain the dominant contributor to variation in EOE and EUE in childhood, regardless of household stress levels, and should therefore be considered key targets for intervention.

\section{ON INFORMATIONAL WITHIN-PAIR CORRELATION}

Andreas Jensen' and Jacob Hjelmborg ${ }^{2}$

${ }^{1}$ Biostatistics, Institute of Public Health, University of Copenhagen, Denmark ${ }^{2}$ Epidemiology, Biostatistics and Biodemography, Institute of Public Health, University of Southern Denmark, Denmark

The within-pair correlation coefficient is used widely for comparing mutual dependence of a continuous outcome observed in MZ and DZ twin pairs. This measure, the product-moment correlation coefficient introduced by K. $\sim$ Pearson in 1904, shortly called the Pearson correlation, underlies all classic twin analysis. The biometric polygenic ADCE model of quantitative genetics providing measures as heritability and environmental effects is formulated in terms of the Pearson correlation, hence the importance for twin analyses can not be overstated. The Pearson correlation is a measure of linear dependence. Deviations from linearity in within pair association and more elaborate functional relationships will not be detected. It is easy to obtain functional relationships for which the Pearson correlation is zero. This also holds for the Spearman rank correlation, the Kendall's tau and other measures used for assessing the degree of similarity within twin pairs. One remedy is to transform the outcome, however, this changes the interpretation and may not be feasible for instance when having multiple outcomes; for example, epigenetic $\mathrm{CpG}$ sites or alike to study. A measure of correlation that equals the Pearson correlation when the outcome is bivariate normal distributed and is zero if and only if the twins are independent is desirable. Such a measure exists: It comes from information theory, was introduced by E. H. Linfoot in 1957 and is highly relevant for studying independence and functional dependence between random variables. Based on the fundamental mutual information of random variables it bridges information theory and statistics. It is hard to estimate in general. We aim to make the informational correlation coefficient operational in twin analysis settings, in particularly when classical correlation measures fall short. We present applications and discuss implications for studies with twin data. 


\section{EFFECTS OF PREGNANCY COMPLICATIONS ON}

\section{OUTCOMES OF TWINS BIRTH}

Olesya Bespalova and Olga Kosyakova

The Research Institute of Obstetrics, Gynecology and Reproductology named after D.O. Ott, St. Petersburg, Russia

Introduction: The frequency of multiple pregnancies varies from 0.7 to $14.6 \%$ worldwide. Perinatal care and delivery of multiple pregnancies are topical problems in modern obstetrics. The reason for it is high level of complications for women and fetuses during gestation, delivery and neonatal periods. In $75-100 \%$ cases multiple pregnancy occurs under the threat of interruption, and as a consequence in developing of placental insufficiency, which in turn leads to premature delivery. In this case, prematurity, low birth weights combined with intrauterine growth retardation are the main factors leading to poor perinatal outcomes in multiple pregnancies. Materials and Methods: We conducted retrospective analysis of the pregnancy duration, labor and perinatal outcomes in 765 cases of twins births in the Research Institute of Obstetrics, Gynecology and Reproductology named after D.O. Ott between 2007 to 2017. Results: The percentage of twins that were delivered in the institute was $2.2 \%$. The ratio of spontaneous and induced pregnancies with twins was 1:3. The ratio of monochorionic and dichorionic types of twins was $1: 10$. Premature birth was diagnosed in $74 \%$ of cases of monochorionic and $58.7 \%$ of dichorionic pregnancies, preeclampsia in $71 \%$ and $45 \%$ respectively, placental insufficiency in $77 \%$ and $47.4 \%$, premature rupture of membrane in $41 \%$ and $30 \%$. In the case of induced twins, complications were observed seven times more often. Vaginal delivery occurred in 189 (25\%) pregnant with twins. A total number of $576(75 \%)$ pregnancies with twins were completed by a cesarean section, 281 (48.7\%) of which were on time, and 295 $(51.3 \%)$ prematurely. The most frequent indications were: placental insufficiency $-42 \%$ and $18 \%$, acute hypoxia of one of the fetus $-27 \%$ and $15 \%$, preeclampsia $-15 \%$. According to the analysis of state of newborn twins, the percentage of children without born asphyxia was $71.3 \%$, while $15.7 \%$ were born in a state of mild asphyxia, $8 \%$ in the average severity of asphyxia and $5 \%$ in severe asphyxia. No significant difference in incidence of twins born vaginally and with caesarean section was revealed. Conclusion: Multiple pregnancies require a differentiated approach to them, depending on the history of the occurrence and the type of placentation. Development of new methods of dynamic testing for pregnant and fetuses may help to predict and reduce perinatal complications.

\section{THORACO-OMPHALOPAGUS CONJOINED TWINS SHARING} THEIR HEARTS

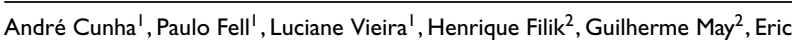
Barbosa $^{2}$, Guilherme Albé ${ }^{2}$, Giovana Sartori ${ }^{2}$, Paulo Zen ${ }^{2}$, and Rafael Rosa ${ }^{2}$

${ }^{I}$ Hospital Materno Infantil Presidente Vargas, Porto Alegre, Brazil

${ }^{2}$ Universidade Federal de Ciências da Saúde de Porto Alegre, Porto Alegre, Brazil

Introduction: Conjoined twins are a rare condition, estimated in 1:75.000 births. Our aim was to report the prenatal findings of a case of thoraco-omphalopagus conjoined twins that shared their hearts. Case Report: The mother was a 31-year-old pregnant woman in her fourth gestation. She was referred to the fetal medicine service due to an ultrassound performed at 12 gestational weeks that found conjoined twins fused by the abdomen. This exam was repeated in the hospital at 14 gestational weeks, showing conjoined twins apparently fused by the thorax and upper abdomen. They apparently shared the heart and liver, however this interpretation was hindered by the early gestational age. There seemed to be only a single umbilical cord, which emerged caudally to the fetuses union. The umbilical arteries were observed emerging laterally to the bladders. It was a monocorionic and monoamniotic gestation. Echocardiography disclosed that the fetus at left had a normal heart and the fetus at right presented dextrocardia. The interventricular septum and the exit ways were not properly visualized. The second trimester ultrassound performed at 22 gestational weeks did not identify other malformations (normal cranium, limbs and spine). Two venous ducts were visualized. Four defined cavities were observed and the hearts were apparently connected by ventricles. The pericardium could not be defined. The echocardiography performed soon after revealed apparent heart communication between the right atrium of the fetus at right and the left atrium of the fetus at left. The fetus at left had tetralogy of Fallot with pulmonary atresia. There was a big interventricular perimembranous communication with a large aorta overriding the trabecular septum in $50 \%$ of its perimeter. Magnetic resonance imaging displayed conjoined twins fused by thorax and superior abdomen, above the umbilical cord insertion. The right hepatic lobe of the fetus at right was related to the left hepatic lobe of the fetus at left. There were two stomachs, two gallbladders and four kidneys. Conclusions: prenatal diagnosis of conjoined twins is very important. This allows the evaluation of the point of attachment and its complexity, for, then, help in the management and prognosis determination.

\section{TWIN PREGNANCY WITH AN ACARDIC FETUS PRESENTING A CONSERVATIVE MANAGEMENT}

André Cunha', Jorge Telles', Henrique Filik ${ }^{2}$, Guilherme May ${ }^{2}$, Eric Barbosa ${ }^{2}$, Guilherme Albé ${ }^{2}$, João Gonzales ${ }^{2}$, Maria Angélica Ferreira ${ }^{2}$, Daniel Arenas ${ }^{2}$, Paulo Zen ${ }^{2}$, and Rafael Rosa ${ }^{2}$

${ }^{I}$ Hospital Materno Infantil Presidente Vargas, Porto Alegre, Brazil

${ }^{2}$ Universidade Federal de Ciências da Saúde de Porto Alegre, Porto Alegre, Brazil

Introduction: Twin reverse arterial perfusion sequence (TRAPS) or acardiac twinning is a very rare condition. Our aim was to report the conservative management of a twin pregnancy with an acardic fetus. Case Report: the patient was a 29-year-old woman referred due to obstetric ultrasound at 14 weeks showing monochorionic and diamniotic twin pregnancy with twin 2 presenting malformation of the upper portion of the body, suggestive of an acardiac fetus. Fetal ultrasound performed at hospital confirmed this diagnosis, that was of TRAPS. After this, she was referred to laser treatment for umbilical cord ablation in a reference service of the country. At evaluation, there were no signs of hemodynamic decompensation in the pump fetus, and, as blood flow to the acardiac fetus was mostly restricted to the umbilical cord, there was no surgical indication at that moment. Second trimester ultrasound performed at 22 weeks revealed a pump fetus without morphological abnormalities, weighing $560 \mathrm{~g}$, and an acardiac fetus, represented by a dysmorphic mass, weighing $165 \mathrm{~g}$. There was evidence of vascularization inside the acardiac fetus through Doppler evaluation. Echodopplercardiogram of the pump fetus was normal. Magnetic resonance imaging did not show alterations of the pump fetus. The acardiac fetus was composed by the lower portion of abdomen and lower limbs, presenting significant edema. Periodic ultrasounds were performed from 26 weeks of pregnancy in order to early diagnose any cardiac decompensation of the pump fetus. The parameters evaluated consisted of the ratio between the weights of the acardiac twin and the pump fetus; cardiothoracic ratio of the pump fetus; tricuspid regurgitation view; presence of polyhydramnios in the pump fetus and ductus venosus Doppler of the pump fetus. These parameters remained normal and it was decided to hospitalize the patient at 30 weeks for ultrasound monitoring. The pump fetus, a girl, was born at 33 weeks of pregnancy weighing 2,125 g and presenting Apgar scores of 8/9. The acardiac fetus weighed 1,090 $\mathrm{g}$ and had an aspect of a shapeless mass with relative more development of the lower limbs. The pump twin presented early respiratory dysfunction. However, it evolved without complications. Conclusions: There are few described cases of TRAPS in the literature with conservative management. We identified some factors that could help in the management of future cases, as early diagnosis and assessment of hemodynamic stability. 


\section{DIZYGOTIC TWINS CONCORDANT FOR} MYELOMENINGOCELE

André Cunha' ', Cristine Dietrich', Vanessa Petersen ${ }^{2}$, Juliana Chinelato ${ }^{2}$, Fernanda Altmann ${ }^{2}$, Elisa Correia ${ }^{2}$, Victória Guimarães ${ }^{2}$, Rosana Rosa ${ }^{2}$, Paulo Zen $^{2}$, and Rafael Rosa ${ }^{2}$

${ }^{1}$ Hospital Materno Infantil Presidente Vargas, Porto Alegre, Brazil

${ }^{2}$ Universidade Federal de Ciências da Saúde de Porto Alegre, Porto Alegre, Brazil

Introduction: Structural defects, including concordant malformations, are more frequently observed in monozygotic than dizygotic twins. Our aim was to describe dizygotic twins concordant for defects in neural tube closure. Case Report: The patient was a 29-yearold pregnant woman when she was referred to the high risk prenatal due to dichorionic diamniotic gestation (concordant with sex), hypothyroidism and fetal spina bifida associated to Arnold-Chiari malformation type II. She denies the use of folic acid supplementation during pregnancy. The previous pregnancy was uneventful. The husband of the patient was a 32-year-old and nonconsanguineous man without history of health problems. There are reports of neural tube defect - in this case, myelomeningocele — in the family (a sister of the woman), who died in the first months of life. Ultrasound examination performed at 22 weeks gestation showed, in both fetuses, lemon sign, flattened cerebellum (banana sign), and lumbosacral myelomeningocele. Ventriculomegaly was observed only in the second twin. Fetal echocardiography of the twins was normal, and fetal magnetic resonance imaging confirmed the findings seen on ultrasound. Children born by cesarean section at 38 weeks of pregnancy. Clinical evaluation of them confirmed the presence of the fetal findings. The additional examination through cerebral ultrasonography showed the presence, in both newborns, of dilated ventricular system. The correction of myelomeningocele occurred in both children in the first day after birth. Conclusions: The association observed in our patients is considered rare. However, we cannot exclude the possibility that the positive family history of defects in neural tube closure, considering a model of multifactorial disease, and the lack of maternal supplementation with folic acid may have contributed to a greater chance of involvement of both fetuses. Events related to the twinning itself, as well as other intrinsic not known genetic factors, cannot be discarded.

\section{MONOZYGOTIC TWINS DISCORDANT FOR CONGENITAL CYSTIC ADENOMATOID MALFORMATION}

André Cunha' , Jorge Telles' , Luciano Targa ', Gisele Calai', Marine Del Maschi' Marco Farina Jnr ${ }^{2}$, Fernanda Altmann², Luciana Beltrão ${ }^{2}$, Paulo Zen², and Rafael Rosa $^{2}$

${ }^{1}$ Hospital Materno Infantil Presidente Vargas, Porto Alegre, Brazil

${ }^{2}$ Universidade Federal de Ciências da Saúde de Porto Alegre, Porto Alegre, Brazil

Introduction: Congenital cystic adenomatoid malformation (CCAM) is a rare anomaly of lung development. Our aim was to report a case of monozygotic twins discordant for CCAM. Case Report: The pregnant woman initially came to the evaluation at 23 weeks of pregnancy due to monochorionic and diamniotic twin pregnancy. First trimester screening had not been made. The patient was a 31-year-old woman in her second pregnancy. Ultrasound examination performed at our hospital showed the presence of polyhydramnios and a lesion in the right lung with numerous small cysts inside in twin 2, that is, a type II CCAM. Both fetuses had weight in the 50th percentile Magnetic resonance imaging (MRI) revealed the presence of a multicystic image in the right lung of twin 2, predominantly of the lower lobe topography, measuring about $4 \mathrm{~cm} \times 3.1 \mathrm{~cm} \times 2.7 \mathrm{~cm}$, apparently affecting over $50 \%$ of the lung volume. There was no significant deviation of the mediastinum. Echocardiography of both fetuses was normal. The following ultrasound examinations showed similar findings. There was no evidence of cardiac decompensation or hydrops in twin 2. Children were born by cesarean section at 36 weeks of gestation.
Both were male. The first twin weighed 2,360 grams and had Apgar scores of 9 in the first and fifth minutes. The second twin presenting type II CCAM had similar measures and Apgar scores: he weighed 2,330 grams and presented Apgar scores of 8 and 9. He clinically evolved uneventful and did not need to undergo surgery during his first weeks of life. Conclusions: Monozygotic twins have an increased risk of structural defects compared with dizygotic twins. CCAM diagnosed during the prenatal period have a good prognosis except in children with large lesions and pulmonary hypoplasia. Large masses can cause mediastinal shift and lead to cardiovascular compromise resulting in fetal hydrops and death. MRI has also been helpful as an important complementation to the ultrasound examination, which can assist both in diagnosis but also in evaluation, for example, of the lesion extension.

\section{PATTERNS IN ORAL GLUCOSE TOLERANCE TEST - ARE THEY HERITABLE?}

Christine Dalgård', Sören Möller ${ }^{2}$, and Kirsten Ohm Kyvik ${ }^{3}$

${ }^{I}$ Department of Public Health, Environmental Medicine, University of Southern Denmark, Odense, Denmark

${ }^{2}$ Odense Patient data Explorative Network (OPEN), Odense University Hospital and Department of Clinical Research, University of Southern Denmark

${ }^{3}$ Department of Clinical Research and The Danish Twin Registry, University of Southern Denmark and Odense Patient Data Explorative Network (OPEN), Odense University Hospital, Odense, Denmark

Introduction: Recent studies demonstrated that there is considerably heterogeneity in glucose response curves during oral glucose tolerance test (OGTT), and this heterogeneity was associated with specific cardiometabolic risk profiles. However, it is unknown whether genetic factors can explain this heterogeneity. Materials and Methods: Data was included from the GEMINAKAR cohort established during 1997-2000 within the population-based Danish Twin Registry where glucose concentrations were measured at three times during a 2-h oral glucose tolerance test following an overnight fast. The GEMINAKAR population consists of 756 complete zygosity determined twin-pairs, aged 18 to 67 years of age and initially free of known diabetes or cardiovascular disease. Heterogeneity of glucose response during OGTT was examined with latent class mixedeffects models, evaluating goodness of fit by Bayes Information Criterion (BIC). Furthermore, we calculated the casewise concordance of twins in a pair belonging to the same predicted latent class, separately for MZ and DZ twins. Results: In total, 1,496 twins had valid glucose concentrations measured from the OGTT (99\% with all three measures). We identified five latent classes with different glucose response patterns and varying between $1 \%(n=13)$ and $38 \%(n=572)$ of the cohort. In total for 53\% (397 of 747) both twins in a pair were assigned into the same class, consisting of a concordance of $62 \%$ (191 of 308) in MZ pairs and 47\% (206 of 439) in DZ pairs. In comparison, the expected class concordance for two unrelated twins in the cohort would be only $32 \%$. The observed casewise concordances (apart from the smallest class, in which no concordant pairs were observed among 13 individuals) varied between $29 \%$ and up to $62 \%$ in total, varying from $38 \%$ to $74 \%$ in MZ twins and $23 \%$ to $53 \%$ in the DZ twins. Conclusions: The five identified classes fit well with studies on singletons in the literature, while the observed higher concordance in MZ compared to DZ and still higher than for unrelated individuals indicate heritability of glucose response patterns. The varying casewise concordances between classes indicate possible differences in heritability between classes. 


\section{PROVIDING INDIVIDUALISED CARE FOR MULTIPLE BIRTH FAMILIES FROM CONCEPTION TO 1 YEAR: DEVELOPMENT OF AN E-LEARNING MODULE FOR HEALTHCARE PRACTITIONERS}

Nathalie Turville, Laura Maguire, Louisa Clifford, and Laura McGrath-Scott

Faculty of Health, Education \& Life Sciences, Birmingham City University, Birmingham, UK

Introduction: The number of multiple births in the United Kingdom has been increasing with just over 11,000 births in 2015 (ONS, 2016). As parents adjust to family life and caring for two or more babies, who may also have been born prematurely, they are faced with many social, emotional, practical and economic challenges. Pregnancy and the transition to parenthood are widely recognised as critical time periods that will influence longer term outcomes for infants and their families. Multiple birth families require practitioners who are both knowledgeable and able to effectively support them, providing the right level of care and support at the right time. In response to this, an e-learning module has been developed to introduce practitioners to the discrete and potentially complex bio-psycho-social needs of multiple birth families, from conception to 1 year of age. Material and Methods: The Elizabeth Bryan Multiple Births Centre (EBMBC) was established in April 2017 arising from the collaboration between Birmingham City University (BCU) and the Multiple Births Foundation (MBF). A core aim of the EBMBC is 'to develop evidence-based education and professional development programs for health care and allied professionals involved in the care of multiple birth families'. A multidisciplinary team within the Faculty of Health, Education and Life Sciences (BCU) was established to develop a foundation module for health care practitioners whose roles bring them in to contact with multiple birth families and who wish to gain further knowledge and skills in caring and supporting them. E-learning is time efficient, cost effective and flexible. The learning activities are designed to promote skills of enquiry, reflection and problem solving within a supportive community of practice. The content draws on current evidence and focuses on four broad areas: (1) An introduction to multiple births: (2) Management of pregnancy, labour and delivery; (3) Care in a neonatal unit; (4) Parenting and caring for multiple babies at home. The experiences of parents of multiples arising from research carried out by the EBMBC form an essential element of the module. Results and Conclusions: As the first of a proposed suite of modules, delivery of the 'The Individualised Care of Multiple Birth Families from Conception to 1 Year' will begin January 2018. Evaluation will be undertaken throughout and following completion to ensure that the EBMBC vision to 'deliver excellence in education, practice and research' is met.

\section{NEONATAL OUTCOMES IN TWIN PREGNANCIES - 10 YEARS' EXPERIENCE OF VAGINAL VERSUS CESAREAN DELIVERY}

Filipa Coutinho, lolanda Ferreira, Sofia Cabrita, Teresa Bombas, Etelvina Fonseca, and Paulo Moura

Obstetric Department, Centro Hospitalar e Universitário de Coimbra, Coimbra, Portugal

Introduction: Twin gestations account for $0.5-2 \%$ of all pregnancies and this rate has been growing as a result of successful assisted reproductive treatments and increasing maternal age. Twin birth is associated with a higher risk of adverse perinatal outcomes than singleton birth, even more when considering monochorionic twins. Recent studies state that there is no evidence of better outcomes for cesarean delivery versus planned vaginal delivery in an otherwise uncomplicated pregnancy. The aim of this study was to evaluate if the mode of twin delivery influences perinatal outcomes, chorionicity considered. Material and Methods: We performed a retrospective study using 581 multiple pregnancies followed in our hospital between 2006 and 2017. We separated the population by chorionicity (monochorionic vs dichorionic) and evaluated neonatal outcomes comparing mode of delivery (vaginal vs cesarean) within each group, using the chi-Square test from SPSS Statistic 22.0. Results: We studied 118 (20.3\%) monochorionic and 463 (79.7\%) dichorionic pregnancies. These groups were similar regarding age and parity, with a mean age of 30.7 years $(17-44)$ in the monochorionic group (G1) and 32.1 years (17-46) in the dichorionic group (G2). Regarding gestational age at birth, $88 \%$ of monochorionic pregnancies were delivered before 37 weeks and only $67.8 \%$ of the dichorionic were preterm $(p<.001)$. When evaluating the great prematurity, both G1 and G2 had similar rates of delivery before 32 weeks of gestational age ( 16.2 vs. $9,9 \%, p=.053)$. Respecting G1, there were $42(35.6 \%)$ vaginal deliveries and $76(64,4 \%)$ deliveries by csection. The difference in admission to neonatal intensive care unit between vaginal and cesarean delivery was not significant (50\% vs. $45,3 \% ; p=.49$ ) and the Apgar scores of $<4$ at first and $<8$ at fifth minute were also similar (0\% vs. $2.6 \%, p=.14$ and $19.5 \%$ vs. $22 \%$, $p=.66$ respectively). Considering dichorionic pregnancies, there were $189(40.8 \%)$ vaginal deliveries and $274(59.2 \%)$ by cesarean section. As we noticed in G1, there was no significant difference in admission to NICU between vaginal and cesarean delivery $(31.7 \%$ vs. $38 \%, p=.052$ ) as well as in the Apgar score $<4$ at first and $<8$ at fifth minute $(1.3 \%$ vs. $3.3 \%, p=.06$ and $18.3 \%$ vs. $17.9 \%, p=$ .89 , respectively). Conclusions: As previous studies demonstrated, our study concludes that, despite the chorionicity, the mode of birth does not influence the primary neonatal outcomes.

\section{GESTATIONAL DIABETES AND MULTIPLE PREGNANCY - A 10 YEAR RETROSPECTIVE OF OBSTETRIC COMPLICATIONS AND NEONATAL OUTCOMES}

Filipa Coutinho, Iolanda Ferreira, Sofia Cabrita, Teresas Bombas, Etelvina Fonseca, and Paulo Moura

Obstetrics Department, Centro Hospitalar e Universitário de Coimbra, Coimbra, Portugal

Introduction: In singleton pregnancies, gestational diabetes (GD) is associated with large for gestational age neonates and adverse perinatal outcomes, including stillbirth and perinatal death. The impact of GD in multiple pregnancies is, however, unclear. The aim of this study is to clarify the impact of GD in multiple pregnancies concerning obstetric complications and neonatal morbidities. Material and Methods: This was a retrospective study, including women with twin pregnancies who attended our service between 2007 and 2016. We considered two groups: Group 1 - Pregnant women with GD, and Group 2 - pregnant women without GD. We compared obstetric and perinatal outcomes between the two groups. Statistical analysis was made using Chi-Square test from SPSS Statistic 22.0. Results: In our 581 patients with twin pregnancies we identified 53 cases of gestational diabetes $(9.1 \%)$. The two groups were similar regarding age and parity, with a mean age of 31.6 years (17-46) in G1 and 33.9 years (26-44) in G2. Regarding obstetric complications, the incidence of hypertension $(2.5 \% \mathrm{G} 1$ vs. $0 \% \mathrm{G} 2)$, preeclampsia $(1.1 \%$ G1 vs. $0 \%$ G2), threatened preterm birth $(29.4 \%$ G1 vs. $28.3 \%$ G2) and fetal growth restriction $(20.1 \% \mathrm{G} 1$ vs. $24.5 \% \mathrm{G} 2)$ was similar in both groups $(p=.06)$. Also, the incidence of delivery before 32 weeks had no significant difference between the two populations in study $(11.2 \%$ vs. $11.5 \% ; p=.937)$. Concerning neonatal outcomes, in the diabetic group, the incidence of Apgar score $<4$ at the first minute was $7.5 \%$ comparing to $1.9 \%$ in the non-diabetic group and this difference was statistically significant $(p=.006)$. Also, the rate for Apgar score $<8$ at the 5 th minute was higher in the diabetic group ( $30.2 \%$ vs. $17.6 \%, p=.21) .37,8 \%$ of the newborns of G1 were admitted to a NICU compared to $38,7 \%$ in G2 $(p=.25)$. Conclusions: Despite the evidence that in singleton pregnancies with GD there is a higher rate of obstetric complications and worst neonatal outcomes, twin pregnancies may not follow the trend. Our study showed that in 
twin pregnancies there is no difference in the complications between diabetic and non-diabetic women and, regarding neonatal outcomes, only the first minute Apgar score $<4$ had a superior frequency (statistically significant) in children from diabetic mothers.

\section{'WHAT ABOUT US?' NEGOTIATING THE REALITIES OF PARENTING MULTIPLES DURING THE FIRST FIVE YEARS}

\section{Laura Maguire}

Faculty of Health, Education and Life Sciences, Birmingham City University, Birmingham, England

Introduction: The aim of this research is to explore the experiences of parents of twins or higher order multiples during the first five years, in order to gain insight and understanding of the reality they make of their lives, behaviors, emotions and interactions There is limited recognition of the voices of multiple birth parents within both literature and family centred health and social care practices. Pressures from contemporary discourses of the 'good' mother and 'involved' father are examined but rather than examining mothers and fathers in isolation the study asks how parents together manage ideals of shared parenting and how their identities as parents of twins or higher order multiples develops. Professionals, popular 'experts', media, family and friends can all provide support to couples when parenting multiples. The study also explores how such advice and support contributes to multiple birth parent's experiences, selfconfidence and identities and whether any advice or support is particularly helpful, or indeed unhelpful. Materials and Methods: The study consists of two phases, utilizing a grounded theory methodology. Ten couples who are first time parents of twins or higher order multiples have been interviewed through the use of loosely structured dyadic and individual interviews. In addition, participatory visual methods, including participant generated diagrams and photograph elicitation have facilitated exploration and discussion of support that parents find enabling and contribute to their family wellbeing. Results: Five emerging themes have been identified, 'powerlessness', 'multiple birth family practices', 'impact of burden' and 'barriers to support and unmet needs'. These themes provide insight into the realities of multiple birth parents during the first five years and the impact this has upon their families' wellbeing. Conclusion: These findings illustrate areas where multiple birth families require support during the first five years and give health and social care practitioners insight into how to best support them.

\section{CHANGES IN EATING BEHAVIORS DURING CHILDHOOD: A STUDY OF TWINS}

\section{Cláudia Ribeiro ${ }^{1,2}$, Andreia Oliveira ${ }^{1,2}$, Carla Moreira ', and Elisabete Ramos ${ }^{1,2}$ \\ ${ }^{1}$ EPIUnit-Instituto de Saúde Pública, Universidade do Porto, Porto, Portugal \\ ${ }^{2}$ Departamento de Ciências da Saúde Pública e Forenses e Educação Médica, \\ Faculdade de Medicina, Universidade do Porto, Porto, Portugal}

Introduction: Childhood is a relevant period to define long-term eating behaviors, thus it is crucial to improve our knowledge on the determinants of eating behaviors during early life. Although the heritability of human behavioral traits is now well established, environmental factors are also believed to be key determinants and it is expected an increase of their influence with growth. Twin samples are the ideal settings to study the potential role of both genetic and environmental factors. To understand the changes in selective eating behaviors between 4 and 7 years old, we compare eating behaviors between pairs of twins. Materials and Methods: The participants are twins from the Generation XXI birth cohort, Porto, Portugal. Information on children's eating behaviors was collected at 4- and 7-year-old by face-to-face interviews applied to a parent, generally mothers. A total of 98 mothers of twins (65 pairs dizygotic and 29 monozygotic) provided information on children's eating behaviors. The questionnaire evaluated if the children: eat too much; refuse soup or fish; ask reward for eating certain foods and impose their food preferences. Comparison of eating behavior changes (difference between 4 and 7 years old) between twins were evaluated by Wilcoxon's test for paired data. Results: In the present study the behaviors with large change between 4 and 7 years old were 'refuse soup or fish' and 'impose their food preferences'. Regarding 'food refusal', $18.7 \%$ of children who did not refuse food at 4 years began to decline soup or fish at 7 years, by the contrary $7.8 \%$ refused at 4 years and did not refuse at 7 years. Regarding 'imposition of food preferences', from the $37.2 \%$ of children who changed their behaviors, $25.0 \%$ began to impose more their food preferences during this period. No significant differences were observed between twins for the changes on any of the eating behaviors traits under study. After stratifying for dizygotic and monozygotic twins, the trend for eating behaviors changes did not differ between pairs and no significant differences were observed. Conclusions: From 4 to 7 years old children acquire more control over their own eating behaviors, perceived by the increment of the desire to impose their food preferences and increase to refuse certain types of food. The changes of eating behaviors during childhood was similar between twins.

Financed by the European Union [HEALS (FP7-ENV-2013603946)] and by PathMob (POCI-01-0145-FEDER-016837).

\section{PERINATAL OUTCOME ACCORDING TO CHORIONICITY IN TWIN PREGNANCY}

Yolanda Cuñarro López, Paula Alonso Ortuño, Santiago García-Tizón Larroca, Ignacio Cueto Hernández, and Juan Antonio De León Luis

Obstetrics and Gynecology Department, Hospital General Universitario Gregorio Marañón, Madrid, Spain

Introduction: The rate of twins has increased over the last years mainly due to the current use of assisted reproductive technology and the results of these pregnancies can be different according to chorionicity. Materials and Methods: A descriptive longitudinal retrospective study of twin deliveries was performed between 20122016 in a tertiary referral hospital in Madrid. We analyzed maternal and peripartum variables as well as perinatal mortality according to chorionicity. Results: Our sample included 1743 newborns from 873 twin pregnancies, $1450(83.05 \%)$ were dichorionic (DC) and 296 (16.95\%) monochorionic (MC). Maternal age mean (DC 35.18 vs. MC $33.68 \mathrm{y}, p<.001$ ), gestational age at delivery (DC 35.56 vs. MC $33.67, p<.001)$, proportion of delivery $<37 \mathrm{w}$ (DC $51.86 \%$ vs. MC $82.43, p<.001),<34 \mathrm{w}(\mathrm{DC} 14.07 \%$ vs. $35.81 \%, p$ $<.001)$ and $<28 \mathrm{w}(\mathrm{DC} 2.62 \%$ vs. MC $7.43 \%, p<.001)$ were different. Any fetal pathology was more common in MC (OR 4.17, $p$ $<.001$ ) and the most frequent complication was IUGR (DC 3.73\% vs. MC $18.84 \%, p<.001)$. Twin-to-twin transfusion sindrome was present in $14.38 \%$ of MC. Differences were seen in mode of delivery; vaginal (DC $51.31 \%$ vs. $43.73 \%, p<.05$ ) and C-section (DC $48.69 \%$ vs. MC $56.27 \%, p<.05)$, but no differences in proportion of C-section of the second twin (DC $49.52 \%$ vs. MC 57.43\%, $p$ $=.013$ ). Mean neonatal birthweight was statistically lower in MC, both in the 1st twin (DC 2,313.6 g vs MC 1,913.5 g, $p<.001$ ) and in the 2 nd one (DC 2,251 g vs. MC $1.905 \mathrm{~g}, \mathrm{p}<0.001$ ) but there were no differences in $\mathrm{pH}$ at birth (1st twin: DC 7.30 vs MC 7.31, $p=.28$; 2nd: DC 7.27 vs. MC 7.29, $p=.010)$. MC presented worse results in Apgar score (1st min $<7$ in 1st twin: DC $15.8 \%$ vs. MC 27.28\%, $p=.002 ; 5 \mathrm{~min}<7$ in 2 nd twin: DC $32.96 \%$ vs. MC $46.16 \%, p<$ .001 ). Neonatal intensive care unit admission (DC $34.16 \%$ vs. MC $59.09 \%, p<.001)$ and length of stay were higher in MC (DC 19.23 days vs MC 31.59 days, $p<.001)$. Perinatal mortality was higher in MC, both crude (OR 3.558, $p<.001)$ and adjusted for twin order (OR 3.559, $p<.001)$. Conclusions: In our sample of twins, monochorionic presented a higher risk of prematurity, lower birth weight and adverse perinatal outcome. 


\section{EFFECTIVENESS OF DINOPROSTONE FOR INDUCTION OF}

\section{LABOUR IN TWINS}

Andrea Agustín Oliva', Marta Benito Vielba ${ }^{2}$, Vanessa Elena Lázaro García ${ }^{3}$, Raquel Crespo Esteras ${ }^{2}$, José Manuel Campillos Maza², and Sergio Castán Mateo²

${ }^{I}$ San Jorge Hospital, Huesca, Spain

${ }^{2}$ Miguel Servet University Hospital, Zaragoza, Spain

${ }^{3}$ Reina Sofía Hospital, Tudela, Spain

Introduction: In recent years an increase in multiple gestations has been observed due to IVF techniques. Vaginal delivery in twins is conditioned by: presentation of the first twin, gestational age, fetal weights and obstetric experience. Fetal-maternal risks in twins at 37-38 weeks are similar to single pregnancies at 42 weeks. When reaching this gestational age, induction of labor is recommended. Previously it may be necessary for medical or obstetric reasons. As advantages, induction decreased fetal morbimortality and as disadvantages, an increased cesarean rate and risk of tachysystole with fetal compromise is described. Pharmacological and mechanical methods for induction of labor exist. The slow release vaginal PGE2 device is a widely used method in single gestations and its use is approved in twin pregnancies. Material and Methods: A descriptive observational, longitudinal and prospective study of controlled twin pregnancies was performed at the Miguel Servet University Hospital, between 1/1/2014 and 5/31/2015. Objective: to determine the effectiveness of slow release vaginal PGE2 device in induction in twins. Inclusion criteria: 1st twin in cephalic presentation, fetal well-being, unfavorable cervical conditions and gestational age $\geq$ 32 weeks. Results: 203 twins delivered in the study period. Applying criteria of inclusion, the sample obtained was 51 patients. After insertion of the device, $52.9 \%$ started labor, $35.29 \%$ required posterior oxytocic induction and only $15.68 \%$ needed a second method of cervical maturation. $78.43 \%$ of pregnant women had a vaginal delivery, $19.6 \%$ had urgent cesarean delivery and a single case required cesarean section of the second twin after vaginal delivery of first twin. The first twin was born by an eutocic delivery in $43.1 \%$ and $35.3 \%$ by vacuum extraction. The most frequent indication for labor induction was to reach 37 weeks of gestation. The appearance of hypertonus and tachysystole with repercussion in fetal cardiotocographic record was $7.8 \%$, in all cases resolved with measures of resuscitation and removal of the device; None of them required urgent cesarean section for this reason. The perinatal outcomes, analyzed in a subsequent subanalysis, were favorable in vaginal delivery group and in the cesarean group. Conclusions: The slow release vaginal PGE2 device is an effective and safe method for the induction of labor in twin pregnancies, achieving a high rate of vaginal delivery without increasing perinatal risks.

\section{NECROTIZING ENTEROCOLITIS IN TWIN PREGNANCIES: CAN WE FIND A KEY IN PLACENTAL ABNORMALITIES?}

R. R. Aapkes' , K. E. A. Hack ${ }^{2}$, C. Koopman-Esseboom ${ }^{3}$, P. G. J. Nikkels ${ }^{4}$, J. B. Derks $^{5}$, and H. A. A. Brouwers ${ }^{3}$

${ }^{I}$ Department of Obstetrics, University Medical Center Utrecht (UMCU), Utrecht, the Netherlands

${ }^{2}$ Department of Gynecology and Obstetrics, Gelre Ziekenhuizen, Apeldoorn, the Netherlands

${ }^{3}$ Department of Neonatology, University Medical Center Utrecht (UMCU), Utrecht, the Netherlands

${ }^{4}$ Department of Pathology, University Medical Center Utrecht (UMCU), Utrecht, the Netherlands

${ }^{5}$ Department of Obstetrics, University Medical Center Utrecht (UMCU), Utrecht, the Netherlands

Objective: We aimed to evaluate the relationship between chorionicity, placental abnormalities and NEC in multiple pregnancies. We hypothesized that unbalanced interfetal transfusion through vascular anastomoses in MC placentation causes hypoperfusion of the intestinal mucosa, increasing the risk of developing NEC. Methods: All women with multiple pregnancies who delivered at our institution between January 1995 and December 2015 were retrospec- tively selected. We compared baseline characteristics and neonatal and maternal outcomes of MC placentation with dichorionic (DC) placentation. Second, we analyzed ultrasound and placental pathology findings of MC multiples with and without NEC. Finally, we compared illness characteristics of NEC in MC multiples with NEC in DC multiples. Results: We included 2859 DC neonates and 817 $\mathrm{MC}$ neonates. NEC occurred significantly more often in MC twins as compared to DC twins (3.3\% and $1.6 \%$ respectively), after correction for birth weight, gestational age and nulliparity (OR 1.7, 95\% CI $[1.0,2.8])$. Ultrasound abnormalities were not associated with NEC. Histopathology however showed that NEC was significantly associated with the presence of unbalanced interfetal transfusion $(76.9 \%$ of MC multiples with NEC versus $31.4 \%$ of cases without NEC, $p=$ $.001)$. MC twins appeared to develop a more severe disease course as compared to DC twins, with $72.7 \%$ of MC multiples and $46.2 \%$ of DC multiples needing surgery $(p=.138)$. Conclusion: NEC is more common in MC multiples as compared to DC multiples, at least in part due to the presence of and related to the presence of unbalanced interfetal transfusion through arterial-venous anastomoses in the placenta. Subtle ischemic damage caused by intra-uterine fetal hypotension or anaemia likely plays a key role in the development of NEC in MC twins.

\section{OUTCOME OF THE SECOND TWIN ACCORDING TO INTERTWIN DELIVERY INTERVAL IN DIAMNIOTIC PREGNANCIES: DOES TIME MATTER?}

Clelia Callegari, Paola Algeri, Salvatore Andrea Mastrolia, Maddalena Incerti, Sabrina Cozzolino, Valentina Giardini, and Patrizia Vergani

Department of Maternal-Fetal Medicine, MBBM Foundation, San Gerardo Hospital, University of Milano-Bicocca, Monza, Italy

Introduction: Optimal management of twin deliveries, in particular regarding intertwin delivery interval, is a matter of debate, in literature is suggested a cut off interval of 30 minutes. We aimed to asses, in diamniotic twin pregnancies after vaginal delivery of the first twin, the outcome of the second twin according to this interval. Materials and Methods: This was a retrospective observational study including diamniotic twin pregnancies delivered at our Institution between 2000 and 2015. We compared the outcomes of the second twin according to intertwin delivery interval considering a latency of 30 minutes as cutoff. Inclusion criteria were: gestational age at delivery $\geq 35$ weeks, birth weight $\geq 1800 \mathrm{~g}$, vaginal delivery of the first twin. Exclusion criteria were: higher twin order, caesarean section of the first twin, fetal malformations and stillbirth. Results: A number of 275 diamniotic twin pregnancies were delivered during the study period: $257(93.5 \%)$ had an intertwin delivery inter$\mathrm{val} \leq 30$ minutes and the remaining $18(6.5 \%)$ had an interval $>30$. Maternal characteristics were similar in both groups, while the rate of preeclampsia was higher in the group with a intertwin delivery interval $>30$ minutes $(7.0 \%$ vs. $27.8 \%, p=.01)$. Of interest, the latency was longer in those women undergoing induction of labor $(55.3 \%$ vs. $78.8 \%, p=.05)$. In addition, vaginal delivery of the second twin was more frequent if the intertwin delivery interval was $\leq 30$ minutes $(89.5 \%$ vs. $50 \%, p=.001)$, while the rate of vacuum delivery or emergency caesarean section were significantly higher for a latency $>30$ minutes (respectively $7.0 \%$ vs. $27.8 \%, p=.01$; $3.5 \%$ vs. $22.2 \%, p=.01$ ). A significantly higher rate of jaundice in the second twin born after $>30$ minutes was observed $(6.6 \%$ vs. $27.8 \%, p=.01)$. No difference was found in the composite neonatal outcome (at least one among neonatal death, respiratory distress syndrome, disseminated intravascular coagulation, patent ductus arteriosus, intraventricular hemorrhage, leukomalacia, retinopathy of prematurity, necrotizing enterocolitis, and neonatal sepsis) as well as in the rate of neonatal hypoxia, between the study groups $(3.5 \%$ vs. $5.6 \%, p=.5$ ). Conclusions: In our study an intertwin delivery interval $>30$ minutes was not associated to an increase in neonatal adverse outcomes for the second twin after vaginal delivery of the 
first one. The optimal intertwin delivery interval cut off should be defined considering fetal wellbeing.

\section{NUCHAL TRANSLUCENCY: AN ULTRASOUND PARAMETER FOR OUTCOMES OF MONOCHORIONIC DIAMNIOTIC TWIN PREGNANCIES?}

lolanda Ferreira, Filipa Coutinho, Sofia Cabrita, Elsa Vasco, and Paulo Moura Obstetrics A Department, University and Hospitalar Center of Coimbra, Portugal

Introduction: Twin monochorionic diamniotic (MCDA) pregnancies are associated with higher frequency of adverse perinatal outcomes, like fetal death, twin-to-twin transfusion syndrome (TTTS) and relevant birth weight discordances. The intertwin discordances between nuchal translucency (NT) measurements during the first trimester screening may be of value in predicting those adverse outcomes. Materials and Methods: The aim of this study was to evaluate the discordance of first-trimester ultrasound nuchal translucency (NT) and its correlation with the outcome of MCDA pregnancies. We present a retrospective study evaluating 117 MCDA twin pregnancies followed in our institution between January 2007 and December 2016. Intertwin discordances of nuchal translucency (NT) was assessed as well as its ability to predict complications during twin pregnancy, such as TTTS and birth weight discordance of $\geq 20 \%$. We determined receiver operating characteristic (ROC) curves using SPSS version 22. Results: Of the 117 cases of MCDA pregnancies, 26 (22\%) had at least one adverse outcome: there was one case $(0,9 \%)$ of fetal death after the first trimester; 15 cases $(12.8 \%)$ of TTTS and 21 cases $(17.9 \%)$ of fetal weight discordance at birth of $\geq 20 \%$. The mean discordance of NT was of $24.8 \% \pm 21.6$ $[0-104.8 \%]$ and about $41.9 \%$ of women $(n=49)$ had a NT discordance superior to $20 \%$ on fetal ultrasound. The area under the curve (AUC) regarding NT discordance was 0.56 for TTTS and 0.55 for birth weight discordance of $\geq 20 \%$. The optimal values for NTdiscordance to predict TTTS were superior to $22.2 \%$ and for predicting birth weight discordance of $\geq 20 \%$ were more than $14.8 \%$. Conclusions: ROC curves regarding NT discordance in this sample showed an AUC $>0,5$, which indicates that this ultrasound parameter can be used to predict the evaluated adverse outcomes in MCDA, in our study TTTS and fetal weight discordance at birth of $\geq 20 \%$.

\section{DOES TWIN PREGNANCY IN OLDER WOMEN HAVE THE} SAME OUTCOMES AS IN YOUNGER WOMEN?

Iolanda Ferreira, Filipa Coutinho, Sofia Cabrita, Elsa Vasco, and Paulo Moura Obstetrics A Department, University and Hospitalar Center of Coimbra, Portugal

Introduction: The number of gestations in older women and the use of assisted reproduction techniques in the same group has shown an increasing tendency in the last decades. It is known that older women with singleton pregnancies have a higher risk of complications during pregnancy and after delivery compared to younger women. However, these outcomes on twin pregnancy with advanced maternal age are still under investigation. Materials and Methods: The aim of this study was to evaluate pregnancy and perinatal outcomes of twin gestations between old and young women groups. We performed a retrospective study evaluating 581 twin pregnancies followed in our institution between January 2007 and December 2016. Women were divided into two groups: $\geq 35$ years (study group [SG]) and $<35$ years (control group [CG]). We assessed population characteristics, complications during pregnancy and postpartum and perinatal outcomes using student t-test and chi-square test with SPSS version 22. Results: A total of 581 twin pregnancies were evaluated (CG: 423 women; SG: 158 women). The mean age in CG was 29.8 [17-34] and in the SG was 37.2 [35-46] years old. There was not a significant difference between both groups regarding the frequency of hypertension (CG: $2.1 \%$; SG: $2.5 \% ; p=.77$ ) and preeclampsia (CG: $1.2 \%$; SG: $0.6 \% ; p=.56$ ) during pregnancy.
The rate of cesarean delivery was of $60.5 \%$ in the CG and $58.9 \%$ in the SG $(p=.72)$. The mean gestational age at delivery was 34,5 weeks [24-38] in the CG and 34.8 weeks [24-38] in the SG ( $p=$ .69). There was no difference between groups regarding labor before 37 weeks (CG: $74.2 \%$; SG $65.8 \%$; $p=.05$ ). However, in this sample, the CG presented a significant higher rate of low birth weight ( $<2500$ gr): $75.8 \%$ vs $65.8 \%(p=.002)$. There were no differences on NICU admission (CG: $39.2 \%$; SG: $34.2 \% ; p=.11$ ) or perinatal death rate (CG: $1.2 \%$; SG: $1.3 \%$; $p=.91)$. There were also no differences concerning post-partum complications, in particular postpartum bleeding (CG: 1.2\%; SG: $1.3 \% ; p=.93$ ) and post-partum anemia (CG: $8.3 \%$; SG: $9.5 \% ; p=.64)$. Conclusions: Maternal age does not seem to have a negative impact in twin pregnancy. In fact, there was no difference in the above-mentioned outcomes between groups, except for low birth weight rate, which was higher in the control group. This probably reflects the tight pregnancy surveillance regarding the SG.

\section{MATERNAL COMPICATIONS IN TWIN PREGNANCY}

Odeta Hoxhaj, Maksim Gjoni, Eneida Dede, and Denisa Bajraktari University Hospital for Obstetrics and Gynecology 'Mbreteresha Geraldine', Tirana, Albania

Background: Twin pregnancy is a high-risk pregnancy as it is associated with significant increase in the perinatal mortality and morbidity and maternal mortality and morbidity. Due to the increased fertility procedures, the number of twin pregnancies has also increased. Materials and Methods: This study was carried out to determine the maternal complications related with twin pregnancy. This was a cross-sectional observational study among all women with twin pregnancy, admitted at University Hospital for Obstetrics and Gynecology in Tirana, Albania during the period January 2013January 2014. Results: Out of 6,875 deliveries, 213 (2.4\%) patients had twin pregnancy; 134 (64.2\%) were multipara and 69 (35.8\%) were primipara. Most of the patients were between 22-29 (67.9\%) years group. The duration of gestations was 32-36 weeks for 60 (32.1\%) patients, 37-40 weeks for $110(56.6 \%)$ patients. Maternal complications were noticed as anaemia in $67(35.8 \%)$ patients, hypertension in $44(22.6 \%)$ patients, post-partum haemorrhage (PPH) in $39(18.9 \%)$ patients, hyperemesis gravidarum in $17(7.5 \%)$, polyhydramnios in $13(5.7 \%)$ patients, antepartum hemorrhage (APH) in $13(5.7 \%)$ patients, premature rupture of membranes (PROM) in $9(3.8 \%)$ patients, gestational diabetes in $13(5.7 \%)$ patients, urinary tract infection (UTI) in $9(3.8 \%)$ patients, complications of operative delivery in $9(3.8 \%)$ patients. There was no maternal death in our study. Conclusions: Twin pregnancy is a high-risk pregnancy associated with significant increase in maternal mortality and morbidity. With aggressive management of complications such as preterm labour, fetal distress, hypertension, $\mathrm{APH}, \mathrm{PPH}$, premature rupture of membranes; morbidity and mortality can be improved.

\section{MONOAMNIOTIC MONOCORIAL MANAGEMENT. CASES AND COMPLICATIONS IN OUR HOSPITAL DURING LAST YEAR}

Oscar Vaquerizo Ruiz, Carmen Gutiérrez-Cecchini Pérez, Jose Adánez García, Ana Escudero Gomis, and Marina Navarro López

Obstetric and Gynecology Department, The Central University Hospital of Asturias, Oviedo, Spain

Introduction: Twin pregnancy occurs in one of every 250 pregnancies. If the division occurs between the 8 th and 13th day, gestation will be monoamniotic monochorionic. This happens in less than $1 \%$ of the twin pregnancies. These gestations present the typical complications of twin gestation, adding the specific ones of monoamnionicity, the most serious of them is the intrauterine fetal death by cord entanglement which appears in most cases. We present three cases of monochorionic monoamniotic twin pregnancies at our center during 
the last year and their complications. Case Reports: The first case was a pregnant woman referred from another center at week 26 for control at a third-level hospital, admitted at week 29 for intensive daily control, and decided to end at week 32 by a pathologic cardiotocographic fetal monitoring of a twin, appreciating in the delivery a great entanglement of both cords. The second case was a pregnant woman controlled in the high-risk obstetrical and prenatal unit of our center, admitted at week 29 for control, objectifying a discordance of fetal weights, so that cesarean section was decided at week 32 , observing a mild entanglement of cords and confirming the difference of weights. The third case was a pregnancy controlled from week 8 , when the diagnosis of monoamninicity was made, with a biweekly ultrasound follow-up, who had a miscarriage of both twins at week 14. Conclusions: Although the obstetric control, the time of hospitalization and the end of gestation remain controversial, the need for correct diagnosis, a biweekly ultrasound follow-up in prenatal and high obstetric care units, is evident. Hospital admission around 28-30 weeks for intensive daily control, as well as ending between $32-34$ weeks by cesarean section, are accorded with by the majority of authors. The high frequency of complications, both early and late, with severe consequences in the majority have to be considered in these gestations.

\section{PREGNANCY OUTCOMES OF MONOCHORIONIC DIAMNIOTIC TWIN PREGNANCIES FROM NATURAL CONCEPTION VERSUS THOSE CONCEIVED BY ASSISTED REPRODUCTION TECHNOLOGIES}

Alba Roca Prats, Julia Escrig Estrems, Joana Dasí Carrasco, Alicia Martinez Varea, Vicente José Diago Almela, and Alfredo Perales Marín

Obstetrics Department, La Fe University Hospital, Valencia, Spain

Introduction: The objective of this study was to evaluate and compare pregnancy outcomes between monochorionic diamniotic (MCDA) twin pregnancies conceived spontaneously and those resulting from assisted reproduction technologies (ART). Whether the mode of conception is associated to different pregnancy outcomes in MCDA twin pregnancies is still unclear. Materials and Methods: A retrospective study was carried out at La Fe's University Hospital (Valencia, Spain). All MCDA twin pregnancies between July 2009 and January 2017 were included. Maternal baseline characteristics, pregnancy complications, as well as perinatal outcomes of these gestations, were reviewed. Results: The study cohort included 210 MCDA twin pregnancies; 182 (86.67\%) were from natural conception (NC) and $28(13.33 \%)$ were conceived by ART. 48 out of $182(26.4 \%)$ pregnancies were from the NC group, and 3 out of $28(10.7 \%)$ gestations after ART had twin-to-twin transfusion syndrome (TTTS; $p=.12$ ); 36 out of the 48 cases (75\%) from NC group, and 2 out of the 3 cases $(66.7 \%)$ from the ART group underwent fetoscopic selective laser photocoagulation (FSLP). 39 out of $182(21.4 \%)$ gestations from NC, and 4 out of 28 (14.3\%) pregnancies after ART had preterm labor $(p=.5)$. The mean gestational age at diagnosis of preterm labor in the NC group was $30+0$ weeks, and in the ART group was $29+1$ weeks. Pregnant women were treated with ritodrine or nifedipine. The mean gestational age at delivery was $33+3$ weeks in the NC group, and $33+1$ weeks in ART group. The overall mean gestational age at delivery was $34+6$ and $34+4$ weeks' gestation in ART and NC gestations, respectively. In 144 out of $182 \mathrm{NC}$ pregnancies (79.12\%), and in 21 out of 28 ART pregnancies $(75 \%)$ both twins were live birth $(p=.8)$. In 7 NC pregnancies $(3.8 \%)$, and in 1 ART pregnancy $(3.6 \%)$ only one twin was a live birth. From all of the live births, in 29 cases from the NC group and in 2 cases from the ART group, both twins were admitted to the NICU. In $23 \mathrm{NC}$ cases and in 3 ART cases one twin was admitted to the NICU. There were $11(3.7 \%)$ neonatal deaths in the NC group and $1(2.3 \%)$ in the ART group $(p=.9)$. Conclusions: MCDA twin pregnancies from NC showed a non-statistically significant higher incidence of TTTS and preterm labor than those pregnancies after
ART. Nevertheless, the mean gestational age at delivery was not different between the study groups. Future studies with a bigger sample size of both groups of patients are required in order to verity these results.

\section{WHEN TWIN ANAEMIA POLYCYTHAEMIA SEQUENCE (TAPS) AND TWIN-TO-TWIN TRANSFUSION SYNDROME (TTTS) COEXIST}

Katarzyna B. Gajewska-Knapik, Nishigandh Deole, and Sarah L. Adams

${ }^{1}$ Fetal Medicine Department, Cambridge University Hospitals, Cambridge, UK

${ }^{2}$ Department of Obstetrics and Gynaecology, The Ipswich Hospital NHS Trust, Ipswich, $U K$

${ }^{3}$ School of Clinical Medicine, Cambridge University Hospitals, Cambridge, UK

Introduction: Twin anaemia-polycythaemia sequence (TAPS), first described in 2006, is caused by minuscule placental anastomoses and arises spontaneously in 3-5\%, or after laser treatment in $13 \%$ of monochorionic pregnancies. It is prenatally diagnosed based on discordant measurements in middle cerebral artery peak systolic velocity (MCA PSV); $>1.5$ multiples of the median (MoM) in one twin and $<0.8$ in the other. More often, a postnatal retrospective diagnosis is made, based on a haemoglobin $(\mathrm{Hb})$ disparity of $8 \mathrm{~g} / \mathrm{dL}$ between anaemic and plethoric twins. An absence of oligo/polyhydramnios sequence distinguishes TAPS from TTTS prenatally. But what is the haemodynamic presentation when these 2 pathologies coexist? Case Report: A 39-year-old woman, G2P1, presented with monochorionic diamniotic (MCDA) twins. There was no significant crownrump length (CRL) or nuchal translucency (NT) discrepancy in the 1st trimester (CRL 64.3 and $61.8 \mathrm{~mm}$; NT $1.4 \mathrm{~mm}$ for both twins). The pregnancy was uncomplicated until 30 weeks and 6 days when $24 \%$ discrepancy in size was found, with oligohydramnios in the sac of the smaller twin 1 (T1) and polyhydramnios in the bigger twin 2 (T2) (deepest vertical pool $2 \mathrm{~cm}$ and $13 \mathrm{~cm}$, respectively). T1 Dopplers were normal. T2 Dopplers showed normal waveform and pulsatility index (PI) in the umbilical artery, but an abnormal flow in the Ductus venosus (DV) with PIV above 95th centile. A very low PI in the MCA and impaired cardiac contractility were also observed in T2. The MCA PSV of T1 was 1.13 MoM and T2 0.9 MoM. Based on abnormal DV Dopplers in T2, a diagnosis of TTTS Quintero stage 3 was made, and the twins delivered by emergency caesarean section. T1 was anaemic at delivery, with Apgar 9,10, 10 (at 1,5 and $10 \mathrm{~min}$ ) and $1430 \mathrm{~g}$ weight. T2 was plethoric and required resuscitation, with Apgar 6,8, 9 and weight $1680 \mathrm{~g}$. T2 spent 3 days on intensive care and 7 days on the high dependency unit. A significant difference in $\mathrm{Hb}$ value was noted at delivery; $10.7 \mathrm{~g} / \mathrm{dL}$ in $\mathrm{T} 1$ and $24.1 \mathrm{~g} / \mathrm{dL}$ in T2. Examination of the placenta revealed a large, pale area belonging to anaemic $\mathrm{T} 1$, and a congested area for plethoric T2. A postnatal diagnosis of pregnancy complicated by TTTS coexisting with TAPS was made. Conclusion: We discuss a case of late presentation of TTTS with coexistent spontaneous TAPS. Although TAPS and TTTS are distinct entities, their characteristic Doppler changes may not always be reflected if the two pathologies coexist, making diagnosis very difficult at advanced gestation.

\section{RISK OF PREECLAMPSIA IN TWIN PREGNANCIES - A} BIRTH REGISTER STUDY

Katariina Laine, Gulim Murzakanova, Kristina Baker Sole, and Sari Räisänen

${ }^{I}$ Oslo University Hospital, Department of Obstetrics, Oslo, Norway

${ }^{2}$ University of Oslo, Norway

${ }^{3}$ University of Helsinki, Finland

Introduction: Preeclampsia is a hypertensive disorder combined with proteinuria and occurs only during pregnancy. The condition is potentially dangerous for the mother and the fetus, and is one of the most common reasons for maternal and foetal mortality and morbidity globally. The etiology is still unknown, but many risk factors are identified. Risk of preeclampsia is higher among nulliparous women 
compared with parous women. Advanced maternal age, obesity, diabetes mellitus and pre-pregnancy hypertension increase the risk of preeclampsia. Materials and Methods: The study is a populationbased registry study. During the years 1999-2014, 930,612 pregnancies and deliveries were recorded in the Medical Birth Registry Norway, and 16,180 of these were twin pregnancies. Results: Overall prevalence for preeclampsia in the entire study population was $3.6 \%$, in singleton pregnancies it was $3.4 \%$ and in twin pregnancies it was $11.8 \%(p=.000)$. The odds for preeclampsia in a twin pregnancy was doubled $(O R=2.16,95 \%$ CI $[1.36,3.41])$ when compared with singleton pregnancy. After adjusting for maternal age and parity, twin pregnancy remained an independent and significant risk factor for preeclampsia (aOR 2.07, 95\% CI [1.31, 3.29]). Conclusions: Twin pregnancy doubles the risk of preeclampsia regardless of parity or maternal age.

\section{CONSENSUS DEFINITION FOR SELECTIVE FETAL GROWTH RESTRICTION IN TWIN PREGNANCY: A DELPHI PROCEDURE}

Asma Khalil, Irene Beune' ', Kurt Hecher, K. Wynia, Wessel Ganzevoort, Keith Reed, Liesbeth Lewi, Dick Oepkes, Eduardo Gratacos, Basky Thilaganathan, and Sanne Gordijn

${ }^{1}$ Fetal Medicine Unit, St George's Hospital, St George's University of London, UK ${ }^{2}$ Department of Obstetrics and Gynecology, University Medical Center Groningen, University of Groningen, Groningen, the Netherlands

${ }^{3}$ Department of Health Sciences, Community and Occupational Medicine, University Medical Center Groningen, University of Groningen, Groningen, the Netherlands ${ }^{4}$ Department of Obstetrics and Gynecology, Academic Medical Center Amsterdam, University of Amsterdam, Amsterdam, the Netherlands

Objective: Twin pregnancies complicated by selective fetal growth restriction (sFGR) are associated with heightened perinatal mortality and morbidity. Inconsistences in the diagnostic features of sFGR hinder the ability to compare or even combine the findings of existing studies. Therefore, it is challenging to establish evidencebased management or monitoring pathways of these pregnancies. The main aim of this study was to determine the diagnostic features of sFGR through a Delphi procedure. Method: A Delphi survey was conducted among an international panel of experts on sFGR. Panel members were provided with a list of literature-based parameters for diagnosing sFGR and were asked to rate the importance of these parameters on a 5-point Likert scale. Parameters were described as solitary parameters (parameters that are sufficient to diagnose sFGR, even if all other parameters are normal) and contributory parameters (parameters that require other abnormal parameter(s) to be present for the diagnosis of sFGR). Consensus was sought to determine the cut-off values for accepted parameters, as well as parameters used in the monitoring, management and assessment of the pregnancy outcome. The questions were presented in two separate categories according to chorionicity. Results: A total of 72 experts were approached, of whom 60 agreed to participate and entered the first round, and $48(80 \%)$ completed all four rounds. For sFGR in monochorionic (MC) twin pregnancy, one solitary parameter (estimated fetal weight (EFW) of one of the twins less than the third centile) and at least two out of four contributory parameters (EFW $<10$ th centile of one of the twins, abdominal circumference (AC) of one twin 95th centile) were agreed upon. For sFGR in dichorionic (DC) twin pregnancy, one solitary parameter (EFW of one twin less than the third centile) and at least two out of three contributory parameters (EFW of one twin less than the 10th centile, EFW discordance of $25 \%$ or more, and UA PI of the smaller twin above the 95th centile) were agreed upon. Conclusion: Consensus-based diagnostic features of sFGR in both MC and DC twin pregnancies, as well as cut-off values for the parameters involved, were agreed upon by a panel of experts. Future studies are needed to validate these diagnostic features before they can be used in clinical trials of interventions.

\section{VAGINAL DELIVERY IN DIAMNIOTIC TWINS, REGARDLESS OF THE CHORIONICITY: FIVE-YEAR EXPERIENCE OF A PORTUGESE HOSPITAL}

Inês Lourenço, Helena Gomes, Inês Rodrigues, Elsa Dias, and Carlos Veríssimo Department of Gynecology and Obstetrics, Hospital Beatriz Ângelo, Loures, Portugal

Introduction: The optimal route of delivery in women with twin gestations depends on the chorionicity, fetal presentation and experience of the clinician. According to the recent guidelines, the planned cesar-ean delivery did not decrease the fetal and neonatal risks, compared with a vaginal delivery. Therefore, a twin gestation in and of itself is not an indication for a cesarean delivery. At our hospital, every women with diamniotic twin gestation, without contraindication for vaginal delivery and whose presenting fetus is in a vertex position, are candidates for a vaginal birth, regardless of choronicity and the presentation of the second twin. Materials and Methods: The purpose of this study was to present the obstetric outcomes of diamniotic twin gestations regarding the route of delivery and neonatal mortality and morbidity of the newborns. It is a retrospective, descriptive study that included all pregnant women with diamniotic twin gestations whose prenatal surveillance and delivery occured in our hospital, between 2012 and 2016. The higherorder multifetal gestations and monoamniotic twin gestations were excluded. Results: This study included 131 diamniotic twin gestations, 102 dichorionic and 29 monochorionic. The median age of the pregnant women was 32 years. As for the delivery route, 51 women had a vaginal delivery, 72 a cesarean and 8 a vaginal birth of the first twin followed by a cesarean for the second twin. Concerning the first twin, there were 33 normal deliveries and 18 operative vaginal deliver-ies. After the birth of the first twin, 43 second fetus presented in vertex, 11 were in breech position and 5 were in transverse lie. The median time between the birth of the first and second twin was 14 minutes. In the second twin's group there were 22 normal births in vertex position; 4 total breech extraction; 6 assisted frank breech deliveries; and 19 operative vaginal deliveries. Regarding the Apgar score, no differences were found between the first and second twins. Conclusion: Results show that the vaginal delivery is a reasonable option and should be considered in uncomplicated diamniotic twin gestations. This route of delivery seems safe for both twins, since this results do not show an increase in the associated perinatal morbidity and mortality. However, a study comparing planned vaginal versus planned cesarean delivery is needed to corroborate these results.

\section{RESEARCHING WITH MULTIPLE BIRTH FAMILIES: AN ETHICAL PERSPECTIVE.}

Nathalie Turville, Laura Maguire, Louisa Clifford, and Laura McGrath-Scott Faculty of Health, Education and Life Sciences, Birmingham City University, Birmingham, UK

Introduction: Ethical principles need to be considered at all stages of a research project, ensuring that the participants are protected and ultimately the research is of benefit to the participant and society (ESCR, 2016). The process of obtaining ethical approval is not uncommonly considered as challenging (Parsell et al., 2014) due to the time-consuming, stressful and complex nature of the review process (Kara, 2012). There are different approaches to identifying ethical issues from using a recognised framework or considering the ethical issues at each stage of the research process (Cresswell, 2013) however different and unanticipated ethical challenges may arise during the conduct of the study. The experience of three doctoral students at varying stages of their studies researching with multiple birth families will be explored. Case Report: Ethical principles: An often overlooked aspect is the dissonance that can be experienced by the researcher arising from the multiple identities that the researcher brings to the study. A trusting and supportive relationship with the 
families is essential to enable exploration of incidents of great significance and emotional impact. For one student, being asked to share personal information about their status as a parent and being presented with scenarios that are incongruent with professional values proved challenging. Process: Traditionally, the National Health Service (NHS) ethical review process is seen to favor a positivist approach to research that does not sit easily with the differing methodologies and more creative methods that are used within qualitative studies. However, for one student, a stimulating and questioning discussion around the use of creative interview techniques provided an unexpectedly positive experience. Practice: A doctoral student, who is also a registered health care professional, experienced tensions that shaped her research with multiple birth families in subtle, yet profound ways. Reflexive attention to these complexities will be explored, which may have resonance for researchers from professional backgrounds. Conclusions: The purpose of this poster is to share our experiences of ethical, iterative researcher reflexivity in the field which has been critical to the progress of our studies with multiple birth families.

\section{PREGNANCY OUTCOMES IN TRIPLET PREGNANCIES}

Joana Dasí Carrasco, Julia Escrig Estrems, Alba Roca Prats, Alicia Martínez Varea, Jose Alberola Rubio, Vicente Jose Diago Almela, and Alfredo Perales Marin

Obstetrics Department, La Fe University Hospital, Valencia, Spain

Introduction: The widespread use of assisted reproduction techniques(ART)has raised multiple pregnancies. Objective: Evaluate the management and outcome of triplet pregnancies. Methods: A retrospective study was carried out,including all women with triplet pregnancies who were admitted at La Fe's University Hospital (Valencia, Spain) between May 2010 and July 2017. Results: 38 patients were included. Mean age: 33 years (range 18-42 years). There were $22(57.9 \%)$ cases from natural conception, and $16(42.1 \%)$ cases from ART; 24 pregnancies $(64.9 \%)$ were dichorionic triamniotic, $9(24.3 \%)$ trichorionic triamniotic, $3(8.1 \%)$ monochorionic triamniotic, and 1 monochorionic diamniotic. Six women were lost to follow-up. Three (7.9\%) had spontaneous abortions. Three had vanishing twin. Three selective fetal embryo reductions were performed. A patient with a monochorionic diamniotic triplet pregnancy desired an elective termination. After the exclusion of these cases, 22 triplet pregnancies were enrolled. Overall management: 5 women $(22.7 \%)$ were prophylactically treated with vaginal progesterone (PG), 1 with elective cervical cerclage (CC) at the end of the first trimester, and $16(72.7 \%)$ received expectant management. The mean gestational age at delivery of the group treated with $\mathrm{PG} / \mathrm{CC}$ was $35+0$ weeks (none underwent preterm labor [PTL]) and $32+4$ weeks the group with expectant management $(p=.006)$. PTL occurred in $11(50 \%)$ women (mean gestational age of 28 weeks $[ \pm 3.01])$. Ten patients received oxytocine receptor antagonist and 1 received nifedipine. Mean age at delivery of women with PTL: $31+2$ weeks vs. $34+6$ weeks of women who did not develop PTL $(p=.007)$. Two patients had twin-to-twin transfusion syndrome.One (stage I) reached 35 weeks. Another (stage II) had stillbirth (21 weeks). One pregnancy developed anemia polycythemia sequence ( 27 weeks), finishing at $31+4$ weeks because of PTL. Mean gestational age at delivery: $33+1$ weeks ( \pm 19 days). Two patients $(9.5 \%$ ) delivered $<28$ weeks, 4 (19\%) 28-31+6 weeks, $3(14.3 \%) 32-33+6$ weeks, 12 (57\%) 34-36+6 weeks. Mean gestational age at delivery of monochorionic-triamniotic pregnancies: $34+3$ weeks; dichorionic-triamniotic: $33+3$ weeks; trichorionictriamniotic: 32 weeks $(p=.51)$ Cesarean section was performed in all patients.Mean birthweight:1,615 g (+/- $463.06 \mathrm{~g})$. No neonatal mortality. Conclusions: PTL was the most common pregnancy complication of triplet pregnancies, entailing an earlier gestational age at delivery. Prophylactic treatment of patients with triplet pregnancies with vaginal progesterone/cerclage associated a later gestational age at delivery.

\section{SECOND TWIN DEMISE IN A MONOCHORIAL BIAMNIOTIC} TWIN PREGNANCY

Marta Ruiz, Begoña Gastón, Natalia Abián, Beatriz Pérez, Irati Zabaleta, Andrea Safont, Julen Barrenetxea, Sonia García, Maitane Urtasun, Marta Bazán, and Itxaso Pedroarena

Obstetrics and Ginecology Complejo Hospitalario de Navarra Pamplona, Navarra, Spain

Introduction: Twin pregnancy is associated with higher rates of complication of pregnancy, the most serious risk being the spontaneous preterm delivery. In addition, monochorionic twins are at risk for complications unique to these pregnancies, such as twinto-twin transfusion syndrome (TTTS), which can be lethal or associated with serious morbidity. For this reason, monochorionic twin pregnancies should be monitored every two weeks. Case Report: A 34-year-old woman undergoing her first pregnancy, a monochorionic biamniotic twin pregnancy, and without any significant medical history. First trimester screening showed low risk for trisomy 21. Closer and accurate follow-up by ultrasound started at week 15 . Two weeks later, it a clear large weight difference existed between both twins (first twin weight in percentile 10 to 25 and second twin weight in percentile lower than 3); as well, as it was not possible to visualize the urinary bladder in the second twin, and there was suspicion of oligohydramnios in this fetus. Doppler examination of both fetuses was normal when monitored and genetic screening was negative. TTTS and growth restriction of second twin was diagnosed; both different diagnosis and therapeutic approachs were explained to the couple and they were referred to the Hospital Clinic of Barcelona. By this time, the second twin had already died. As the patient was zero negative, she was given anti-D immunoglobulin. Afterwards, both a fetal neurosonography and magnetic resonance imaging were realized in week 20 , obtaining normal results. As the living fetus was in breech presentation, an external cephalic version was undergone at term without success. A cesarean section was performed at week 39 . A healthy male was born weighing 3,110 grams with Apgar 9/10 and $\mathrm{pH} 7.14$ and 7.26 (arterial and venous respectively). Conclusions: Early ultrasound examination is crucial in order to provide an accurate follow-up of twin pregnancies; not only to estimate the gestational age, but particularly to determine the chorionicity and amnionicity. This is critical because monochorionic twins have a shared fetoplacental circulation, which puts them at risk for specific serious pregnancy complications, such as TTTS and twin anemia-polycythemia sequence. These complications increase the risk for neurologic morbidity and perinatal mortality compared with dichorionic twins.

\section{PREOPERATIVE ANEMIA-POLYCYTHEMIA IN TWIN-TWIN TRANSFUSION SYNDROME: INCIDENCE, PLACENTAL CHARACTERISTICS AND NEONATAL OUTCOME}

Lisanne S. A. Tollenaar' ', Femke Slaghekke', Johanna M. Middeldorp', Monique C. Haak' , Frans J Klumper' , Enrico Lopriore ${ }^{2}$, and Dick Oepkes'

${ }^{1}$ Department of Obstetrics and Fetal Therapy, Leiden University Medical Center, the Netherlands

${ }^{2}$ Department of Neonatology, Leiden University Medical Center, the Netherlands
Detands

Introduction: Unbalanced inter-twin blood transfusion in monochorionic twin pregnancies may lead to twin-twin transfusion syndrome (TTTS) and twin anemia-polycythemia sequence (TAPS). Whereas TTTS is characterized by a rapid transfusion through large placental anastomoses resulting in twin oligohydramnionpolyhydramnion sequence (TOPS), TAPS is a result of slow transfusion through miniscule anastomoses, leading to anemia in the donor and subsequent polycythemia in the recipient, without signs of TOPS. Although these complications are classified as two separate twin transfusion disorders, there are reports on the presence of anemia-polycythemia (AP) in TTTS twins. However, the true incidence of AP is insufficiently investigated. The aim of this study was 
to evaluate the incidence of preoperative AP in a large population of TTTS twins and to compare maternal, placental and neonatal characteristics between TTTS twins with AP to twins with TTTS alone. Materials and Methods: All TTTS cases treated with laser between 2003-2017 were screened for the presence of AP preoperatively. AP was defined as a middle cerebral artery — peak systolic velocity (MCA-PSV) $>1.5$ multiples of the median (MoM) in one twin and 0.5 MoM. Results: In total, 58/320 (18\%) TTTS twins with complete MCA-PSV records prior to laser fulfilled the criteria for AP. There was no difference in maternal age, parity and Quintero stage between the TTTS-alone and the TTTS-AP group. TTTS twins with AP were more likely to receive laser treatment at a higher gestational age than the TTTS-alone group $(p=.000)$, and their placentas were characterized by fewer vascular anastomoses $(p=0.002)$. The chance of intrauterine fetal demise and neonatal death was the same for both groups, $p=.787$ and $p=.121$ respectively. Conclusions: Preoperative AP is detected is $18 \%$ of the TTTS twins treated with laser. TTTS twins with AP were treated with laser at a significantly higher gestational age and showed fewer vascular anastomoses compared to twins with TTTS alone, corresponding with the pathogenesis of TAPS that is characterized by a later onset of the disease and the presence of only a few anastomoses.

\section{TAPS TRIAL: FETOSCOPIC LASER SURGERY VS. STANDARD CARE FOR TWIN ANEMIA-POLYCYTHEMIA SEQUENCE - AN OPEN LABEL RANDOMIZED CONTROLLED TRIAL}

Lisanne S. A. Tollenaar, Femke Slaghekke, Johanna M. Middeldorp, Monique C. Haak, Frans J. Klumper, Enrico Lopriore, and Dick Oepkes

Department of Obstetrics and Fetal Therapy, Leiden University Medical Center, the Netherlands

Introduction: Monochorionic twins share a single placenta and are connected to each other through inter-twin vascular anastomoses, allowing the blood to transfer bidirectionally between the two fetuses. Unbalanced net inter-twin blood transfusion can lead to twin anemia-polycythemia sequence (TAPS). TAPS is a newly described form of chronic and slow inter-twin blood transfusion characterized by large inter-twin haemoglobin differences without signs of amniotic fluid discordances. TAPS can occur spontaneously or after laser surgery for twin-to-twin transfusion syndrome. Management options include fetoscopic laser surgery of the vascular equator at the placental surface, intra-uterine blood transfusion with or without partial exchange transfusion, selective feticide, delivery and expectant management. The best treatment option for TAPS is not clear and preliminary data are based on case reports and small cohort studies. Design: We propose to conduct a multi-center open-label randomized controlled trial to assess if fetoscopic laser surgery improves the outcome of TAPS compared to standard care. We will randomly assign 140 monochorionic twin pregnancies diagnosed with TAPS between 20-28 weeks of gestation to the fetoscopic laser surgery group or the standard care group, using a web-based application with a computer-generated list with random permuted blocks, stratified by gestational age at inclusion (20-24 weeks vs. 25-28 weeks) and TAPS type (spontaneous vs. post-laser TAPS). The inclusion period will be $2.5-3$ years. Outcome: Primary outcome will be a composite of perinatal mortality and severe neonatal morbidity; secondary outcomes include hematological complications, parental anxiety and long-term neurodevelopmental outcome at the corrected age of 2 years.

\section{PUERPERAL COMPLICATIONS AFTER INDUCTION OF} LABOR WITH DINOPROSTONA IN TWIN GESTATIONS

Andrea Agustín Oliva', Marta Benito Vielba ${ }^{2}$, Sara Agustín Oliva ', Rebeca Herrero Serrano ${ }^{2}$, Jose Manuel Campillos Maza², and Sergio Castán Mateo ${ }^{2}$ ${ }^{1}$ San Jorge Hospital, Huesca, Spain

${ }^{2}$ Miguel Servet University Hospital, Zaragoza, Spain

Introduction: The World Health Organization describe a greater risk of maternal, gestational and puerperal complications in twin pregnancies. An increased risk of mortality, hypertensive disorders, gestational diabetes, instrumental delivery and postpartum haemorrhage, need for transfusion, admission to the intensive care unit and puerperal hysterectomy has been reported. Cesarean delivery associate with increased puerperal morbidity. Induction of labor can reduce complications from a planned cesarean section, however there is an increased puerperal risk if case of urgent cesarean section. Material and Methods: A descriptive observational, longitudinal and prospective study of controlled twin pregnancies was performed at the Miguel Servet University Hospital between 1/1/2014 and 5/31/2015. Puerperal complications were compared after induction of labor with dinoprostone in the group that ended by vaginal delivery and cesarean section. Inclusion criteria: 1st twin in cephalic presentation, fetal well-being, unfavorable cervical conditions and gestational age $\geq 32$ weeks. A sample size of 51 patients was obtained. Results: The group ended by vaginal delivered had $40 \%$ of puerperal complications and this percentage reaches $63 \%$ in case of cesarean section, although this difference was not statistically significant $(p=.163)$. The most frequent puerperal complications were: moderate anemia ( $\mathrm{Hb} 7-9.9 \mathrm{~g} / \mathrm{dl})$ in $21.6 \%$, mild anemia ( $\mathrm{Hb} 10-10.9 \mathrm{~g} / \mathrm{dl}) 5.9 \%$ and severe anemia $(\mathrm{Hb}<7 \mathrm{gr} / \mathrm{dl})$ in $5.9 \%$. Other less frequent complications were: puerperal fever, endometritis, perineal tear III / IV and episiotomy infection. Nulliparous women had a sigifitively high risk of complications $(52.3 \%$ vs. $11.1 \% p=.024)$. There was no increased risk in obese women (50\% vs. $43.58 \% p=.666)$. Patients undergoing some assisted reproduction technique had an increased risk $(56.2 \%$ vs. $23.52 \%$, $p=.028 \%$ ).Conclusions: Nulliparous women undergoing assisted reproductive techniques had an increased risk of postpartum complications following twin pregnancy. The risk of postpartum hemorrhage is increased due to uterine distension and this risk increases after induction of labor. Twin pregnancies are risky pregnancies and it is necessary to take prophylactic measures (directed delivery of the placenta and uterotonics) and an early treatment in case of postpartum haemorrhage.

\section{COMPLICATIONS OF DICHORIONIC TWIN PREGNANCIES}

M. Benito Vielba' , M. Choliz Ezquerro ${ }^{2}$, R. Laborda Gotor ${ }^{2}$, A. Agustin Oliva ${ }^{3}$, C. De Bonrostro Torralba ${ }^{4}$, and J.M. Campillos Maza ${ }^{4}$

${ }^{\prime}$ Obstetrics Department, Miguel Servet University Hospital, Zaragoza, Spain ${ }^{2}$ Obstetrics Department, Miguel Servet University Hospital, Zaragoza, Spain ${ }^{3}$ Obstetrics Department, Miguel Servet University Hospital, Zaragoza, Spain ${ }^{4}$ Obstetrics and Gynecology Dept, San Jorge Hospital, Zaragoza, Spain

Introduction: Multiple pregnancies, when compared with single pregnancies, are more likely to present maternal and fetal complications: birth defects, preterm delivery, intrauterine growth restriction, hypertensive disorders and gestational diabetes. Materials and Methods: We collected data from twin pregnancies assisted at our center from January 2013 to June 2017, analyzing feto-maternal complications. The aim of this report is to describe the frequency of these complications in dichorionic twin pregnancies in comparison to single gestations. Results: During the study period, we assisted 604 twin deliveries; $0.7 \%$ monochorionic-monoamniotic, $14.1 \%$ monochorionic-diamniotic and $85.2 \%$ dichorionic-diamniotic. Up to $14.7 \%$ of them were complicated with a gestational diabetes; $10.4 \%$ controlled exclusively by diet, $4.3 \%$ required insulin for the correct glucose control and $0.2 \%$ pregestational diabetes. The 
prevalence of gestational diabetes in single pregnancies is approximately $10 \%$. Another frequent complication is hypertensive disease. It complicated $11.9 \%$ of these pregnancies; $4.7 \%$ expressed as a preeclampsia, $4 \%$ as transient hypertension, $0.4 \%$ as chronic hypertension and $0.4 \%$ were complicated by eclampsia or HELLP syndrome. The estimated prevalence of hypertensive disorders in single pregnancies ranges from $1 \%$ to $5 \%$. Attending to fetal growth restriction, it was noted in $3 \%$ and $5.2 \%$ of the first and second twins respectively. The average gestational age at diagnosis was 31.3 and 28.8 weeks of pregnancy respectively. The prevalence of IUGR in single pregnancies is $3-5 \%$. The most frequent complication of twin pregnancies is prematurity. We observed that the $2.9 \%$ of these deliveries occurred before 28 weeks of gestation, $12.5 \%$ between 28 and 34 weeks, $30.8 \%$ between 34 and 37 weeks and the other $53.7 \%$ over 37 weeks of pregnancy. Global prematurity fluctuates around 9.5\%.With regard to postpartum complications we noticed that uterine atony appeared in the $6.3 \%$ of our patients; $37.5 \%$ of them were resolved only with medical treatment, $43.8 \%$ required the application of a Bakri balloon, 3.1\% needed an embolization of uterine arteries, $12.5 \%$ required compression sutures, and $3.1 \%$ required a hysterectomy. Conclusions: Multiple pregnancies are exposed to a high risk of feto-maternal complications. The early detection and prevention of risk factors allows the adoption of preventive measures with the aim of reducing the morbidity associated to multiple gestations.

\section{COMPLICATIONS OF MONOCHORIONIC TWIN \\ PREGNANCIES}

M. Benito Vielba' , A. Agustin Oliva ${ }^{2}$, M.J. Puente Luján ${ }^{3}$, M. Roca Arquillue ${ }^{3}$, C. De Bonrostro Torralba ${ }^{3}$, and J.M. Campillos Maza ${ }^{3}$

${ }^{I}$ Obstetrics Dept, Miguel Servet University Hospital, Zaragoza, Spain ${ }^{2}$ Obstetrics and Gynecology Dept, San Jorge Hospita, Huesca, Spain

${ }^{3}$ Obstetrics Dept, Miguel Servet University Hospital, Zaragoza, Spain

Introduction: Multiple pregnancies are more likely to present fetal and maternal complications compared to single gestations. Monochorionic pregnancies present a greater risk than dichorionic due to the presence of vascular anastomosis between both fetuses. Materials and Methods: We collected data from twin pregnancies from 2013 to June of 2017 assisted at our center, analyzing feto-maternal complications. The aim of this report is to describe the frequency of this feto-maternal complications in monochorionic pregnancies compared to single pregnancies. Results: During the study period, we assisted 604 twin deliveries; $0.7 \%$ monochorionic-monoamniotic, $14.1 \%$ monochorionic-diamniotic and $85.2 \%$ dichorionic-diamniotic. Up to $10.1 \%$ of them were complicated with gestational diabetes; $5.6 \%$ with gestational diabetes controlled exclusively by diet and $4.5 \%$ required insulin for the correct glucose control. The prevalence of gestational diabetes in single pregnancies is $10 \%$ approximately. Another frequent complication is the hypertensive disease. It complicated the $10.2 \%$ of pregnancies; $4.5 \%$ expressed as a preeclampsia and $5.7 \%$ of transient hypertension. It is estimated that the prevalence of hypertensive disorders in single pregnancies ranges from $1 \%$ to $5 \%$. Attending to the fetal growth restriction it was noted in $2.2 \%$ and $3.4 \%$ of the first and second twins respectively. The average gestational age at diagnosis was 26 and 29 weeks of pregnancy respectively. The prevalence of IUGR in single pregnancies is $3-5 \%$. With regard to prematurity, we observed that $4.5 \%$ of these deliveries occurred before 28 weeks of pregnancy, $21.3 \%$ between 28 and 34 weeks, $30.3 \%$ between 34 and 37 weeks, and the other $43.9 \%$ over 37 weeks of pregnancy. Global prematurity fluctuates around $9.5 \%$. With regard to postpartum complications we noticed that uterine atony appeared in 3 patients; two of these were treated only with medical therapy and one of them needed an embolization of uterine arteries. With respect to specific complications of monochorionic pregnancies, we observed that $30.6 \%$ of cases suffered twin-to-twin transfusion syn- drome; $80.7 \%$ of these patients were treated by anastomosis coagulation and the rest, $19.3 \%$, by cord occlusion. Conclusions: Monochorionic pregnancies are exposed to a high risk of maternal-fetal complications. The early detection and prevention of risk factors allows the adoption of preventive measures with the aim of reducing the morbidity associated to multiple gestations.

\section{BULGING MEMBRANES IN MULTIPLE PREGNANCY}

J. Escrig Estrems, J. Dasí Carrasco, A. Roca Prats, A. Martínez Varea, J. Alberola Rubio, V. J. Diago Almela, and A. Perales Marin

Obstetrics Department, La Fe University Hospital, Valencia, Spain

Introduction: Cervical insufficiency leads to mid-trimester losses or preterm births. Objectives: To evaluate pregnancy outcomes of women with multiple pregnancy and bulging membranes(BM) without intraamniotic inflammation (IAI, defined by IL6 $<2.9 \mathrm{ng} / \mathrm{ml}$ ), compared with those with IAI, with and without microbial invasion of the amniotic cavity(MIAC). Methods: All women with multiple pregnancy and BM admitted into La Fe University Hospital between 2010 and 2016 were included. Patients underwent an amniocentesis of the first twin to quantify amniotic glucose, leukocytes, IL6, leukocyte esterase levels and for microbiological culture. Results: Sixteen patients were included. One $(6.3 \%)$ was a trichorionic triamniotic triplet pregnancy, and $15(93,8 \%)$ were twin pregnancies (1 [6.3\%] monochorionic-diamniotic, 14 [87.5\%] dichorionic). None had a history of miscarriage at 14-24 weeks or preterm delivery. All patients underwent an amniocentesis. The 3 patients without IAI underwent a rescue cerclage. Thirteen had IAI. Median gestational age at diagnosis of patients without IAI: $22+1$ weeks ( \pm 24 days) versus with IAI $21+6$ weeks ( \pm 19 days; $p=.89$ ). Gestational age at delivery of those without IAI: $35+6$ weeks $( \pm 19$ days), median interval from diagnosis until delivery: 98 days $( \pm 32)$, versus women with IAI $23+6$ weeks ( \pm 32 days), median interval until delivery: 14 days $( \pm 22 ; p=.001$ and $p<.001$, respectively). There was 1 delivery at term and 2 preterm in those without IAI, and 5 preterm deliveries and 8 pregnancy losses in the IAI group $(p=.013)$. No statistical differences were found between women with IAI with (4) and without MIAC (9). The ROC curve for amniotic IL6, glucose and leukocyte concentration was determined to assess the diagnostic accuracy of each marker for having a good prognosis (interval of at least 10 weeks between diagnosis and delivery). The IL6 showed the highest diagnostic accuracy, with an area under the ROC curve of 0.927; the best cut-off point was $2.75 \mathrm{ng} / \mathrm{ml}$. Conclusions: Multiple pregnancies with BM without IAI have a higher gestational age at delivery and a longer interval diagnosis-to-delivery than those with IAI.Rescue cerclage may have improved the pregnancy outcome in those without IAI. No different gestational age at delivery has been found between patients with IAI with and without MIAC. We reaffirm, as in singleton pregnancies, that IL6 is the amniotic fluid marker with the highest diagnostic accuracy to determine IAI. The best cut-off point associated with good prognosis was of 2.75 $\mathrm{ng} / \mathrm{ml}$.

\section{DELAYED-INTERVAL DELIVERY: REPORT OF THREE CASES}

M. Benito Vielba', V. Pallares Arnal ${ }^{2}$, R. Herrero Serrano ${ }^{2}$, A. Agustín Oliva ${ }^{2}$, C. De Bonrostro Torralba ${ }^{3}$, and J.M. Campillos Maza ${ }^{2}$

${ }^{1}$ Obstetrics Dept, Miguel Servet University Hospital, Zaragoza, Spain

${ }^{2}$ Obstetrics Dept, Miguel Servet University Hospital, Zaragoza, Spain

${ }^{3}$ Obstetrics and Gynecology Dept, San Jorge Hospital, Huesca, Spain

Introduction: Delayed delivery is sometimes performed in selected multifetal pregnancies when the first twin birth occurs inevitably at the limit of viability. The aim of this procedure is to improve the prognosis and decrease the morbidity and mortality of the second twin. Case Report: We report three cases of delayed interval delivery of dichorionic-diamniotic twin pregnancies assisted at our center between 2015 and 2017. After the first twin delivery, we 
performed a high ligation of the umbilical cord and the second twin was left in utero. The patient received tocolytic therapy and antibiotics and remained hospitalized. Cervical cerclage was never performed due to the current controversy regarding the use of this technique in this clinical context. Our patients were admitted between $21+3$ and $23+6$ weeks of gestation due to a premature rupture of membranes of the first twin, syntomps of preterm birth and cervical incompetence, respectively. The birth of the first twin occured between $24+0$ and $25+4$ weeks. We achieved an average interval delivery of 6.33 days (1-14). The second twin delivery occured between $24+1$ and $26+1$ weeks. The average birth weight of the first and second tiwn was $740 \mathrm{~g}$ and $746 \mathrm{~g}$, respectively. We obtained a survival rate of $34 \%$ for the first twin and $34 \%$ for the second twin. The average stay of the first and second twins that were admitted to the Neonatal Intensive Care Unit (NICU) was of 72 days (28-116) and 39.5 days (12-67) respectively. The first twin birth was vaginal in all cases while the second twin delivery was performed by cesarean section in two out of our three patients. Our neonatal results are not favorable, probably due to the extreme prematurity. Investigation in this field is necessary to promote an accepted protocol for the management of these cases. The maternal postpartum went by without complications, excluding one mother who was readmitted due to a infection of the surgical wound requiring intravenous antibiotic therapy. Conclusion: Delayed delivery of the second twin before 28 weeks of gestation can be an alternative for the obstetrician since it could prolong the pregnancy until a gestational age that confers a better prognosis and a better perinatal outcome for the second twin. This obstetrical procedure is not exempt from complications, so it should be performed in selected cases in which there is no contraindication, and after having informed the patient of risks, benefits, and possible alternatives.

\section{BALLOON TAMPONADE IN TWINS}

Andrea Agustín Oliva ${ }^{1}$, Marta Benito Vielba ${ }^{2}$, Beatriz Diaz Rabasa ${ }^{2}$, Pilar Andrés Orós ${ }^{2}$, Belén Rodriguez Solanilla ${ }^{2}$, and Sergio Castán Mateo ${ }^{2}$

${ }^{I}$ San Jorge Hospital, Huesca, Spain

${ }^{2}$ Miguel Servet University Hospital, Zaragoza, Spain

Introduction: Postpartum hemorrhage (PPH) is the leading cause of maternal mortality worldwide. Several guidelines for PPH management include intrauterine balloon as an effective option when uterotonic drugs are not effective. Twin gestations have an increased risk of PPH due to uterine overdistension since the uterine volume reaches 10 liters. Objectives: The main objective was to determine the effectiveness of intrauterine balloon for management of PPH in our hospital in cases where medical treatment failed and to assess whether twin pregnancies are a risk factor for $\mathrm{PPH}$, its incidence and the effectiveness of the intrauterine balloon in these cases. Materials and Methods: A retrospective descriptive study of patients who required balloon intrauterine $(n=89)$ to stop the PPH. Data about maternal and fetal characteristics, medical treatment and risk factors for PPH were collected during the period between July 2010 and February 2015 from the clinical history. Results: During the study period there were a total of 19,586 births at our hospital; 1,566 PPH and 89 women required balloon taponade $(0.46 \%)$. These patients had a mean age of 33 years (21-45), delivery at 38 weeks (25-42), and their newborns had an average weight of 3,149 $\mathrm{g}(890-4,850$ g). The following risk factors for PPH were found: multiple gestation $27 \%(n=24)$, induction labor $39 \%(n=35)$, delivery expulsive period prolonged $(>3 \mathrm{~h}) 16.9 \%(n=15)$ and did not present risk factors $37.1 \%(n=33)$. The more frequent type of delivery was eutocic $(51.68 \%)$ followed by cesarean section $(31.46 \%)$ and instrumental (28.85\%). $61.8 \%$ of the patients required blood transfusion. As medical treatment oxytocin and rectal misoprostol were used in all of them, prostaglandins in 50.6\% $(n=45)$ and methylergometrine $80.9 \%(n=72)$. The balloon was effective in $95.5 \%$ of patients $(n=85)$ and four patients required the rescue method.
There were no maternal deaths. In twin pregnancies that required the balloon, this was effective in $87.5 \%$ of the cases $(n=21)$; two patients required an embolization and in one case uterine capitonation was performed. $70 \%$ of twins were delivered vaginally delivery, and $20.8 \%$ by urgent cesarean section and planned cesarean section. Conclusions: Recognizing risk factors for PPH optimizes the treatment. The Bakri balloon is an effective technique to treat PPH unresolved with uterotonics. In most cases it prevents the need for surgery or embolization. Multiple gestations are a risk factor for postpartum hemorrhage, despite vaginal delivery.

\section{MAJOR DEPRESSIVE DISORDER AND NAUSEA AND VOMITING DURING PREGNANCY. SHARED GENETIC FACTORS?}

Lucia Colodro-Conde' , Baptiste Couvy-Duchesne ${ }^{1,2}$, Penelope Lind', Jodie Painter ${ }^{1}$, Margie Wright ${ }^{2}$, Grant Montgomery ${ }^{2}$, Dale Nyholt ${ }^{3}$, and Sarah E. Medland'

${ }^{1}$ QIMR Berghofer Medical Research Institute, Brisbane, Australia

${ }^{2}$ University of Queensland, Brisbane, Australia

${ }^{3}$ Queensland University of Technology, Brisbane, Australia

Introduction: Nausea and vomiting in pregnancy (NVP) affects $\sim 70 \%$ of pregnant women to different degrees. Around $14 \%$ of pregnant women experience severe NVP and this progresses to hyperemesis gravidarum (HG) in around $1-3 \%$. NVP and $\mathrm{HG}$ are more prevalent in twin pregnancies as well as in pregnancies involving female fetuses. Women who experience severe NVP and HG have higher rates of major depressive disorder (MDD) prior to the index pregnancy. We therefore hypothesized that there is a genetic correlation between NVP/HG and depression. Materials and Methods: To test our hypothesis, we examined the extent to which individual differences in the occurrence of NVP, HG and the severity of NVP can be predicted by a polygenic risk score (PRS) derived from the most recent Psychiatry Genomics Consortium MDD analyses (PGC-MDD2, leave QIMR out). Data on NVP were obtained from two studies undertaken at the QIMR Berghofer (Australia), $(N=$ 1,440 unrelated women, age at time of survey of 48, range: 26-78). The phenotypes were: (1) presence of NVP for 7 days or more, (2) degree of severity of NVP (5 point scale), and (3) presence of severe NVP, characterized by disruption of daily routine, prescription of medication, loss of weight, and intravenous feeding (proxy for HG). Polygenic risk scores were calculated using the PLINK profile score method for clumped SNPs (thresholds of $p \leq 0.5 * 10-8$, $.1 * 10-5, .001, .01, .05, .1, .5$ or 1$)$. Logistic and linear regressions on the profile scores were performed, controlling for ancestry, age and age 2 at survey time, cohort, and whether it was a twin pregnancy. Results: Among the women from our sample, in at least one of their pregnancies, $53.1 \%$ had symptoms of NVP for 7 or more days and $18.7 \%$ had severe symptoms. After fitting covariates, the MDD PRSs predicted the probability of presenting NVP and the degree of severity (all PRS under threshold $<0.001$ ), and very severe NVP or HG (all PRS under threshold 0.05 ) at a nominally significant level. However, only MDD-PRS $<0.1$ predicting the degree of severity of NVP survived multiple correction testing. Conclusions: There was a signal of the genetic risk for MDD (independent of the time at which any depressive symptoms manifest) predicting NVP. However, it did not survive multiple correction testing. Our results had a power limitation. We expect to increase the sample size in the next few months and to contribute to the understanding of the comorbidity between depression and NVP. 


\section{INCREASED RISK OF PREMATURE MENOPAUSE IN KOREAN WOMEN WHO HAD CHILDBIRTH DURING KOREAN WAR (1950-1953)}

Seung-Ah Choe ${ }^{1,2}$ and Joohon Sung 3,4

${ }^{I}$ Department of Obstetrics and Gynecology, CHA university, Gyunggi-do, South Korea ${ }^{2}$ Department of Epidemiology, Brown University School of Public Health, Providence, RI, USA

${ }^{3}$ Department of Epidemiology, School of Public Health, Seoul National University, Seoul, South Kored

${ }^{4}$ Institute of Health and Environment, Seoul National University, Seoul, South Korea

Introduction: Premature menopause (defined as spontaneous menopause before age of 40 years) is known to be associated with increased risk of cardiovascular disease, osteoporosis and neurodegenerative disorders. Malnutrition or stress during peripartum period is suggested to have a life-long effect on reproductive function. The purpose of this study was to explore the possible effect of childbirth during wartime on the timing of natural menopause. Materials and Methods: Data from the Korean Genome and Epidemiology Study (KoGES) conducted from 2004 to 2013 were used. Among the women who reported their menstrual status, those who naturally experienced menopause before age of 40 years were classified to have premature menopause. Women were determined to have a childbirth during the Korean War when the time of her first or last childbirth was between 1950 and 1953. Potential confounding factors such as early menarche, parity, level of education, early onset of smoking, alcohol use, and year of birth were included in the analysis. $R e$ sults: A total of 109,928 Korean women aged 45 years or older were included in the analysis. Among 75,557 postmenopausal women, $85.1 \%$ reported naturally experienced menopause. The prevalence of premature menopause and having first delivery during the Korean War was $5.0 \%$ and $5.1 \%$ respectively. In the multivariable analysis, having childbirth during the Korean War was related with higher risk of premature menopause in Korean (odds ratio $[O R]=2.61,95 \%$ CI]1.90, 3.57]). Conclusion: Childbirth experience during wartime is associated with higher risk of premature menopause. This finding may contribute to the understanding of the impact of war on the reproductive span of women.

\section{ASSOCIATION BETWEEN FECAL MICROBIOME AND COLON ADENOMAS AND HYPERPLASTIC POLYPS IN MONOZYGOTIC TWINS}

Wendy Cozen', Yang Yu', Amie Hwang', Bing Ma ${ }^{2}$, Laura Buchanan', Guoqin Yu ${ }^{3}$ Anna Wu', James Buxbaum' ', Heinz-Josef Lenz', Thomas M. Mack', Joshua Millstein ${ }^{1}$, and Jacques Ravel ${ }^{2}$

${ }^{I}$ Keck School of Medicine of USC, University of Southern California, Los Angeles, California, USA

${ }^{2}$ School of Medicine, University of Maryland, Baltimore, Maryland, USA

${ }^{3}$ National Cancer Institute, National Institutes of Health, Bethesda, Maryland, USA

Introduction: Colorectal cancer (CRC) arises from adenomatous and serrated colon polyps that are identifiable with colonoscopy. We conducted a study to determine whether fecal microbiota composition and structure are associated with colon adenomas and hyperplastic polyps in a set of MZ twins, controlling for genome. Materials and Methods: 95 individual MZ twins representing 55 pairs from the California Twin Program provided a stool sample and completed questionnaires. Of these, 56 (representing 38 twin pairs) had documented recent colonoscopy and no gastrointestinal diseases, cancer or recent antibiotic use. Body mass index (BMI) and food frequency information was collected 15 years before stool collection and again at the time of stool collection. The V4 region of the $16 \mathrm{~S}$ rRNA gene was sequenced using the HiSeq 2500 system. Alpha diversity measures including number of OTU (operational taxonomic units) and Shannon index, beta diversity and relative abundance of specific taxa were calculated for each sample. Mixed ANOVA models with a random effect for twin pair status adjusted for BMI and family history of CRC were used to examine the association of alpha diversity with polyp status. Results: 8 twins had an adenoma and 8 had a hyperplastic polyp at last colonoscopy; the remainder had no polyps. We found that alpha diversity was higher in twins with adenomas compared to twins with no polyps. When restricted to subjects with colonoscopy in the 3 years prior to stool collection, differences were stronger and statistically significant (mean OTUs for subjects with adenoma, hyperplastic polyps and no polyps $=908$, 850 , and 801 , respectively; $p$ trend $=.018$ ). Beta diversity was lower among randomly paired subjects with adenomas compared to randomly paired subjects with no polyps $(p<.05)$. We also confirmed the reported relative deficit of Bacteroides (genus) and Akkermansia muciniphila and a higher abundance of Fusobacterium associated with adenomas. Conclusion: We found increased richness but more phylogenetic similarity among subjects with adenomas compared to those with no polyps. The timing of measurement of fecal microbiota years after colonoscopy obscures the causal relationship between adenoma development and fecal microbiome, but suggests there are persistent differences in microbiota even after polyps are removed.

\section{ARE MONOCHORIONIC TWINS MONOZYGOTIC?}

Catherine Derom ${ }^{1,2}$, Evert Thiery ${ }^{3}$, Kristien Roelens $^{\prime}$, Steven Weyers' ${ }^{1}$, and Robert Vlietinck ${ }^{2}$

${ }^{I}$ Department of Obstetrics and Gynaecology, Ghent University Hospitals, Ghent, Belgium

${ }^{2}$ Centre of Human Genetics, University Hospitals Leuven, Leuven, Belgium

${ }^{3}$ Department of Neurology, Ghent University Hospital, Ghent, Belgium

Introduction: Traditionally, monochorionicity in multiple pregnancies is associated with monozygosity and opposite-sex twins with dizygosity. For same-sex dichorionic twins one has to rely on genetic markers to confirm their zygosity. Recently, an increasing number of cases of dizygotic monochorionic twins has been described. Most of these twins are from opposite sex and the result of assisted reproductive technologies (ART). Material and Methods: Between 1964 and 2016, placentation, zygosity and the use of ART have been recorded in the population-based East Flanders Prospective Twin Survey. Placentation was recorded by postnatal examination and, between 1964 and 1989, confirmed by histological examination of the dividing septum of the two chorionic sacs. Results: In a consecutive series of 2,006 monochorionic pairs and 3,156 opposite-sex twins all but one of the monochorionic twins were of the same sex and all but one of the opposite-sex twins were dichorionic. The pregnancy of the opposite-sex monochorionic twin was spontaneously conceived. No vascular anastomoses could be detected after injection of the placental vessels. Follow-up data were available in 3 random series of 499 monochorionic pairs. Phenotypically and/or according to a similarity questionnaire the diagnosis of monozygosity could be confirmed in all cases. In addition, blood group typing and/or DNA analysis of tissue samples collected on the maternal side of the cotyledons of each member of the pair in 670 random monochorionic placentas, showed one pair with more than one discordance. The pregnancy of this pair was the result of an artificial induction of ovulation without in vitro fertilization. No vascular anastomoses could be detected after injection of the placental vessels. Conclusion: From these data one can conclude that the monozygosity diagnosis based on the structure of the fetal membranes can be considered accurate in almost all instances and that a monochorionic placenta remains an excellent predictor of monozygosity. 


\section{MINIMIZING IATROGENIC MULTIPLE BIRTHS IN THE UK}

\section{Jane Denton' and Valerie Peddie ${ }^{2}$}

${ }^{1}$ Multiple Births Foundation, Queen Charlotte's and Chelsea Hospital, London, UK

${ }^{2}$ Aberdeen Maternity Hospital, University of Aberdeen, Aberdeen, Scotland

Introduction: Since the conception of Louise Brown in 1978, advances in assisted reproductive technologies (ARTs) have resulted in excess of 5 million births worldwide. Multiple births pose the single biggest risk from IVF; multiple pregnancies being concomitant with increased obstetric and neonatal morbidity and mortality, usually related to preterm delivery. In 2011, the European Society for Human Reproduction \& Embryology (ESHRE) published data from 32 countries on assisted reproductive technology (ART), which highlighted the risk of extremely preterm birth rates, including singleton, twin and triplet deliveries. It is now widely recognised that these risks to both mother and child are unacceptable. In 2004, 24\% of all IVF pregnancies (about 1 in 4) in the United Kingdom resulted in a multiple birth. Following public consultation, the regulatory body - the Human Fertilisation \& Embryology Authority (HFEA — introduced a policy supported by professional bodies and patient groups requiring all clinics to develop multiple birth minimisation strategies enabling a year on year reduction in iatrogenic multiple pregnancy rates to a maximum of $10 \%$ (over 4 years) without compromising success rates. Materials and Methods: The HFEA convened an Expert Group to review and recommend ways to reduce iatrogenic twin pregnancies. In 2009, the HFEA applied an outcome based regulatory policy whereby each clinic was required to develop a Multiple Births Minimisation Strategy, advocating elective single embryo transfer (eSET). Results: As a result, by 2013, multiple pregnancy rates (MPRs) had reduced significantly from 1:4 live births in 2008 , to $1: 6$, and by $2016,1: 7$, and the majority of clinics have met and maintained their target $(<10 \%)$. Despite the reduction in MPR's, pregnancy and live birth rates were sustained. The HFEA has recently started reporting live birth rates "per embryo transferred', which has facilitated continued advocacy of safe clinical practice and eSET. Conclusion: Multidisciplinary involvement has been key to the successful implementation of eSET policies. The way in which the information and data are presented - coupled with acceptance and belief in the regulatory functions - will ensure maintenance of effective eSET programs. Most importantly, collaborative efforts in the UK have resulted in a downward trend of iatrogenic multiples, while improving obstetric outcome for both mother and baby.

\section{CONFRONTING GRIEF: IMPLICATIONS OF TWIN DEATH RITUALS ON THE SURVIVING TWIN IN BOTSWANA}

\section{Senzokuhle Doreen Setume}

Department of Religious Studies, University of Botswana, Gaborone, Botswana

Introduction: Since time immemorial, a number of rituals have been practiced on the surviving twin after the death of other twin. Taking my personal experience as a starting point, the purpose of this article is to explore the process of twin grief in Botswana in the last 10 years. It seeks to explore their grief journey and experiences. It will investigate what rituals are taking place today and explore the extent to which twin grief rituals have assisted their process of grief. Materials and Methods: This was a qualitative study that combined auto-ethnography and phenomenology. The researcher as a twin who has lost their twin-sister is a participant as well. In-depth interviews were carried out with four twins who have lost their twin brothers or sisters. All research protocols were followed. Consent was given through a signed consent form. Results: The study found that the following rituals are still taking place: surviving twin not allowed to view the deceased, lying in the coffin or grave-side lying, not participating in the funeral by the surviving twin, exchange of clothes between the dead and living twin (as the surviving twin lies in the grave or by its side, they take-off their t-shirt and leave it in the grave or beside it, then wear that for the deceased that they find placed by the grave. Conclusions: The results are that surviving twins report mixed feelings: while some participate in the rituals others do not; while some report that the rituals are traumatic, the complicated process of grief. One surviving twin reports that the rituals are processes of comfort. The study recommends that the beliefs that informs the said rituals and practices needs to be understood and then obliterated in order to enable twins to grief effectively.

\section{GENOME-WIDE ANALYSIS OF BUCCAL DNA METHYLATION WITH THE METHYLATIONEPIC BEADCHIP IN MONOZYGOTIC TWINS CONCORDANT OR DISCORDANT FOR AGGRESSION}

Jenny van Dongen', Erik E. Ehli ${ }^{2}$, Fiona A. Hagenbeek', Peter J. Roetman ${ }^{3}$, Toos C.E.M. van Beijsterveldt' ${ }^{\prime}$, Gonneke Willemsen' ${ }^{\prime}$, Robert R.J.M. Vermeiren ${ }^{3}$, Bastiaan T. Heijmans ${ }^{4}$, Meike Bartels', Gareth E. Davies ${ }^{2}$, and Dorret I. Boomsma' ${ }^{1}$ Department of Biological Psychology, VU Amsterdam, Amsterdam, the Netherlands ${ }^{2}$ Avera Institute for Human Genetics, Sioux Falls, USA

${ }^{3}$ Curium-Leiden University Medical Center, Department of Child and Adolescent Psychiatry, Leiden, the Netherlands

${ }^{4}$ Molecular Epidemiology Section, Leiden University Medical Center, Leiden, the Netherlands

Introduction: The Illumina HumanMethylation450 BeadChip (HM450) has been widely applied to measure genome-wide DNA methylation and was recently succeeded by the MethylationEPIC BeadChip (EPIC). The ACTION project (Aggression in Children: Unraveling gene-environment interplay to inform Treatment and InterventiON strategies; http://www.action-euproject.eu/) will measure buccal DNA methylation with EPIC arrays in $>1000$ children, including monozygotic (MZ) twin pairs concordant or discordant for aggression and a clinical sample. Before initiating the main study, we aimed to (1) validate the EPIC array in comparison to the HM450 array for buccal DNA; (2) compare MZ twin correlations for DNA methylation at probes assessed by EPIC compared to probes assessed by HM450; and (3) perform preliminary analyses of buccal DNA methylation and aggression. Materials and Methods: Samples from $105 \mathrm{MZ}$ twins (58\% males, mean age at DNA sampling $=7$, range $=1-10$ ) from the Netherlands Twin Register were measured on EPIC at the Avera Institute of Human Genetics. Based on aggressive and rule breaking behavior at age 3, 7, 10 and 12, assessed with the Child Behavior Checklist (CBCL) by the mother and father, MZ pairs were classified as concordant high $(N=15)$, concordant low $(N=29)$ or discordant $(N=8)$. For 2 twins, technical replicate measures on EPIC were obtained. For 10 samples, methylation had been assessed before with HM450. Quality control (QC) and normalization was performed in meffil; 102 samples passed QC (49 MZ pairs). For each methylation site, the Pearson correlation between MZ twins was computed $(N=$ 789,852 methylation sites, $N=49$ pairs). Results: Correlations between samples across all probes were high for technical replicates (rho $=0.9919$, and rho $=0.9933$, respectively), for matched samples based on common probes on the HM450 and EPIC (mean rho $=0.9817$, range $=0.9794-0.9831$ ) and for samples from MZ twins on EPIC (mean rho $=0.9891$, range $=0.9717-0.9947$ ). Correlations for MZ twins correlated negatively with within-pair difference in predicted $\%$ of buccal epithelial cell $(r=-.60)$. MZ twin correlations for individual sites were higher and displayed a bimodal distribution for EPIC probes (mean $r=.31$ ) compared to probes common to EPIC and HM450 (mean $r=.27$ ). Conclusions: Results indicate high reproducibility between common probes on HM450 and EPIC and greater heritability of methylation levels at novel EPIC probes. Next steps using this dataset include association analyses for aggression. 


\section{GENETIC EFFECTS INTEGRATING THE LAYERS OF PERSONALITY: A TWIN STUDY OF ADOLESCENT OF PERSONALITY TRAITS AND VALUES}

Ariel Knafo-Noam, Louise Twito, and Hila Segal

Psychology Department, The Hebrew University of Jerusalem, Israel

Values are abstract goals, serving as standards to guide behaviors and to evaluate actions, persons, and the self (Schwartz, 1992), whereas personality traits are 'dimensions of individual differences in tendencies to show consistent patterns of thoughts, feelings and actions' (McCrae \& Costa, 1990). Thus, values and traits can be seen as two separate layers of personality, with traits describing what people do, and values describing the motivational aspect of behavior (McAdams, 2015). Indeed, research has found consistent associations between traits and values (Parks-Leduc et al., 2015). For example, extraversion related positively to openness to change and power values. Because values guide behavior, the origin of the values-traits association could reflect the effect of values; however, values also justify behavior, and individuals' values could reflect their self-perception of behavior (Roccas et al., 2002). We therefore employed a genetically-sensitive design to address the origins of the values-traits association. Monozygotic $(N=145$ pairs $)$ and dizygotic ( $N=255$ pairs) 11-year-old twins rated their personality traits with the Big-Five Inventory (John et al., 2001) as part of the Longitudinal Israeli Study of Twins (Avinun \& Knafo, 2013). Adolescents also rated their values with Schwartz's Portrait Values Questionnaire, and ranked them with the Picture-Based Values Survey (Doring, 2010). Phenotypic correlations between values and traits replicated the findings found previously with an adult Israeli sample. Multivariate analyses indicated that most of the association between traits and values reflected overlapping genetic effects. We conclude that common underlying genetic factors give rise to both values and traits. A longitudinal genetic design is needed to further assess whether the genetic effects operate independently on both kinds of variables, or alternatively that genetic effects on one variable give rise to the other variable, across development.

\section{GENE-ENVIRONMENT CORRELATIONS AFFECTING CHILDREN'S EARLY DELINQUENCY AND AGGRESSIVE PLAY BEHAVIORS}

Lisabeth Dilalla and David Dilalla

Southern Illinois University School of Medicine, Carbondale, USA

Introduction: Some early-influenced adolescent delinquents can be biologically based and early developing (continuous antisocials; DiLalla \& Gottesman, 1989). My early work with Irv Gottesman led me to explore childhood influences that affect aggression and delinquency through biological as well as social mechanisms. Besides parenting, child peer play behaviors are a primary influence on child socialization. Gene-environment correlations (rGE) are difficult to assess, but reactive rGE are likely an important aspect of peer interactions. It is likely that young children's transactional influences during play are driven in part by their genotypes through mechanisms of rGE. Thus, early peer interactions may include genotypeevoked behaviors, which may be reflected in play behaviors. Materials and Methods: Twins were assessed at age 5 during a peer play task. Children were paired randomly with an unfamiliar, same-age, same-sex child, thus controlling for parent- and child-chosen environments (passive and active rGE). Twins played separately from each other with unmatched children. Play behaviors were independently, reliably coded for aggressive, commanding, compliant, and difficult behaviors. Children also were independently rated by parents for aggressive and rule-breaking problem behaviors at age 5, and some children were rated again on these behaviors $2-10$ years later. Results: We have earlier shown that children at genetic risk for aggression were more likely to be treated aggressively by un- familiar peers during the peer play (DiLalla \& John, 2014). Further analyses suggest that children at genetic risk for early delinquency/rule breaking are more likely to have partners who behave more aggressively (Spearman's $\mathrm{r}(214)=.19, p=.004$ ), suggesting this is evoked behavior during play. Preliminary evidence also suggests that those at genetic risk of delinquency may elicit more commanding and positive enjoyment from their unfamiliar peers. Some evidence of early bloomers emerged based on early difficult temperament and parent-rated delinquency significantly predicting later delinquency. Conclusions: Early work considering precursors to delinquency suggests the importance of genetic as well as family and peer influences. Here, preschool children's peer interactions added to the equation as important systemic variables. Children's play, which is one of the most important influences on early development, requires further study from an rGE perspective.

\section{PARENTS' EXPERIENCES OF PARENTING PRETERM MULTIPLES}

\section{Louisa Clifford}

Birmingham City University, Westbourne Road, Birmingham, United Kingdom

The poster will set out the research proposal for a study exploring the experiences of parents of multiples born before 37 weeks gestation using a grounded theory approach. Over $50 \%$ of twins and almost all triplets are born before 37 weeks gestation and these account for approximately $15-20 \%$ of all admissions to neonatal units (Royal College of Obstetrics and Gynaecology, 2011). The proposed research will explore the experiences of parents of multiples born before 37 weeks gestation. The overall aim of the study is to further understand how does the preterm birth of multiples impacts parents' experiences, their transition to parenthood and their parental, couple and family relationships during the first year. The study will consist of two phases incorporating semi structured and photo elicitation interview techniques. A constructivist grounded theory approach will be used to obtain relevant narratives from parents, establish an understanding of parents' experiences of raising multiples and 'give voice' to their perspectives (Charmaz, 2006). Parents will be interviewed together to establish a greater insight to their dyadic parenting experiences. They will also be asked to take one photograph per week while their babies are in hospital and for 4 weeks after discharge; the photographs will be used during a photo elicitation interview in phase 2 of the study. This research is required to establish the health and social care needs of families with preterm multiples for services to provide more effective, tailored support and improve the long-term outcomes for families with multiples.

\section{DELAYED-INTERVAL DELIVERY IN DICHORIONIC TWIN} GESTATIONS: A SINGLE-CENTER EXPERIENCE

S. García Francés, I. Jiménez Ubieto, N. Abián Franco, L. Martinez Jiménez, B. Gastón Moreno, B. Pérez Munárriz, M. Ruiz García, I. Zabaleta Loinaz, and C. Larrañaga Azcárate

Complejo Hospitalario de Navarra, Pamplona, Spain

Introduction: To evaluate obstetric and perinatal outcome in twin pregnancies managed with delayed-interval delivery. Materials and Methods: It was performed a retrospective review of all cases of attempted delayed-interval delivery from 2006 to 2015 in a teaching hospital. Results: We found 8 cases of twin gestations managed with delayed delivery after the spontaneous delivery of the first fetus. All pregnancies were dichorionic diamniotic less than 32 weeks. Exclusion criteria were: suspected fetal distress, congenital abnormalities or preterm rupture of membranes in second twin; severe metrorrhagia after first twin delivery; signs of chorioamnionitis or maternal desease. Mean maternal age was 35 years. Up to $75 \%$ of pregnancies were conceived after assisted reproductive techniques. Mean reasons for admission were: threatened preterm labour (37\%) and 
preterm rupture of membranes $(63 \%)$. Mean gestational age at admission was 25 weeks. Delivery of both fetuses was delayed in up to $75 \%$ of cases, achieving a prolongation of 17.33 days on average (from 3 to 64 days). Mean gestational age of first twin delivery was 181 days. All first fetuses were delivered vaginally without episiotomy, and administration of antibiotics and atosiban was continued. We performed expectant management during the first our after first twin delivery in order to assure second twin well-being. Mean first fetal weight was 910 grams (from 290 to 1515 grams). Mortality rates for first fetus achieved 50\% (25\% occurred in previable gestational age before 24 weeks). It was performed cervical cerclage after first twin delivery in two cases $(19+3$ and 20 weeks), prolonging in 20 days and 14 days respectively without attaining viability (second twin delivery at $22+2$ and 22 weeks). For all other cases, clinical surveillance was carried out and no maternal complications were registered during delayed-interval delivery. Mean interval was 9 days (from 1 to 20 days). Second twin delivery took place spontaneously in all cases, vaginally in up to $75 \%$ and by cesarean delivery in two cases due to breech presentation. Delivery of the placenta and postpartum recovery occurred normally in all cases. Conclusions: From our experience, delayed-interval delivery is a reasonable option in pregnancies at an early gestational age in which only the first fetus spontaneously delivers and it should be encouraged in tertiarylevel hospitals in order to prolonging gestational age and reducing morbidity and mortality.

\section{NEONATAL RESULTS OF THE SECOND TWIN DEPENDING ON THE TYPE OF VAGINAL DELIVERY}

\section{Benito Vielba' , B. Diaz Rabasa ${ }^{2}$, M. Rodrigo Rodriguez ${ }^{2}$, A. Agustin Oliva ${ }^{3}$, C. De Bonrostro Torralba ${ }^{2}$, and J. M. Campillos Maza ${ }^{2}$

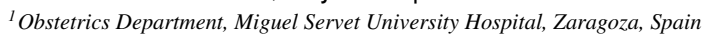 ${ }^{2}$ Obstetrics Department, Miguel Servet University Hospital, Zaragoza, Spain ${ }^{3}$ Obstetrics and Gynecology Department, Hospital of San Jorge, Huesca, Spain}

Introduction: The aim of this study is to analyze the neonatal results according to the type of vaginal delivery of the second twin, comparing the breech extraction with any other type of vaginal delivery. Materials and Methods: We collected data of 555 twin deliveries attended at our center between 2007 and 2017 using these inclusion criteria: gestations $>32$ weeks and vaginal delivery of both twins. A descriptive study was performed with normal quantitative variables expressed as mean and standard deviation, and nonnormal variables expressed as median and interquartile range. Qualitative variables were expressed as percentages. We performed a comparative study using chi-square for categorical variables and Student $t$ and U-Mann-Whitney for quantitative variables. We considered $p<.05$ as statistically significant. Results: The average maternal age was $35.39 \pm 4.882$ and $34.03 \pm 5.144$ in the breech extraction group and in the non-breech extraction group respectively ( $p=$ $.04)$. The gestational age median and the interquartile range was $260(52)$ and 259(52) $(p=.242)$ respectively. The $48 \%$ and $50.5 \%$ were nuliparous women in the breech extraction and in the nonbreech extraction group respectively $(p=.683$, OR $0.90,95 \%$ CI [0.56.1.46]). We found a statistical difference when analyzing the incidence of gestational diabetes with a higher incidence in the breech extraction $\operatorname{group}(p=.006$, OR $2.55(95 \%$ CI $[1.28,5.08])$ and in the spontaneous termination of the pregnancy, which was more frequent in the non-breech extraction group ( $p=.034$, OR $1.68,95 \%$ CI $[1.03,2.74])$. There was no difference in the incidence of hypertensive disease $(p=.326$, OR $0.64,95 \%$ CI $[0.26,1.56])$. According to the neonatal results, there was no difference in $\operatorname{birthweight}(p=$ .446), average interval between twins $(p=.062)$, score in Apgar test at 5 minutes $(p=.193, O R=3.12,95 \%$ CI $[0.51,19.03])$ and arterial blood $\mathrm{pH}<7.15$ ( $p=.519$, OR $1.28,95 \%$ CI [0.60, 2.74]). On the other hand, we found a statistical difference in the Apgar test score $<4$ at the first minute $(p=.002$, OR $6.58,95 \%$ CI $[1.39$, 24.89]), which was more frequent in the breech extraction group.
Conclusions: Vaginal delivery of the second twin by breech extraction performed by trained obstetricians is a safe technique. While it is associated with a lower Apgar score at the first minute, there is no difference in the Apgar score at 5 minutes and in the arterial blood $\mathrm{pH}$ so we can conclude that this technique is not associated with worse neonatal outcomes compared to other types of vaginal delivery.

SOCIAL STRAIN AND IRRITABLE BOWEL SYNDROME: HOW ARE THEY RELATED?

May-Bente Bengtson', Julia Kutschke', and Jennifer Ruth Harris ${ }^{2}$

${ }^{I}$ Medical Department, Vestfold Hospital Trust, Tonsberg, Norway

${ }^{2}$ Department of Genetic research and Bioinformatics, The Norwegian Institute of Public Health, Oslo, Norway

Introduction: Irritable bowel syndrome (IBS) is a prevalent and painful gastrointestinal disorder for which symptoms include abdominal pain associated with abnormal bowel habit. Negative social interactions are associated with IBS, but little is known about the nature of these relationships. The aim of this study was to examine models of causal versus non-causal effects underlying the relationship between IBS and a measure global social strain (GSS). Materials and Methods: The data are based on questionnaire responses received from 5,442 Norwegian twins aged 40 to 80 years. The sample includes 1,986 complete pairs and 1,470 single responders. The questionnaire included a checklist of 43 illnesses and symptoms, including IBS, several psychosocial factors and measurements of social relationships with co-twin, spouse/partner, family and friends. The GSS reflects overall social strain as assessed in each of these four relationships. We used the discordant twin design to explore the nature of the association between IBS and GSS and test for causal effects. Results: The relative risk of having IBS, given a positive report of IBS in the co-twin was $2.72(1.87,3.95)$ among MZ pairs and $1.37(0.83,2.28)$ among DZ pairs. The relative risk of experiencing a high level of GSS given the co-twin reports a high level of GSS was $2.86(2.28,3.58)$ among $\mathrm{MZ}$ and $1.98(1.57,2.50)$ among DZ pairs. In our sample, 168 monozygotic (MZ) pairs and 173 dizygotic (DZ) twin pairs were discordant for IBS. The odds of experiencing high GSS, given a history of IBS, was significant among pairs of unrelated individuals $(O R=2.56,95 \%$ CI $[2.11,3.10])$. However, a high level of GSS was not associated with IBS among twins from $\mathrm{MZ}(O R=1.25,95 \% \mathrm{CI}[0.77,2.03])$ or $\mathrm{DZ}(O R=1.53,95 \% \mathrm{CI}$ $[0.94,2.48])$ pairs. The sample included $249 \mathrm{MZ}$ and $252 \mathrm{DZ}$ pairs discordant for a high level of GSS. The odds of IBS, given a high level of GSS, was significant among pairs of unrelated individuals $(O R=1.93,95 \%$ CI $[1.60,2.32])$. However, IBS was not associated with a high level of GSS among twins from MZ $(O R=1.18$, $95 \% \mathrm{CI}[0.71,1.96])$ or $\mathrm{DZ}(O R=1.59,95 \% \mathrm{CI}[0.92,2.71])$ pairs. Conclusion: Our pattern of results suggests that the association between IBS and GSS is not a causal one, but reflects shared familial and genetic factors.

\section{GENOTYPE SPECIFIC FOR MONOZYGOTIC TWINS DISCORDANT FOR ANTI-THYROGROBULIN AUTOANTIBODY (TGAB) POSITIVITY}

Mikio Watanabe ${ }^{1,2}$, Yoichi Takenaka ${ }^{2,3}$, Chika Honda ${ }^{2}$, Osaka Twin Research Group $^{2}$, and Yoshinori Iwatani ${ }^{1,2}$

${ }^{1}$ Department of Biomedical Informatics, Osaka University Graduate School of Medicine, Osaka, Japan

${ }^{2}$ Center for Twin Research, Osaka University Graduate School of Medicine, Osaka, Japan

${ }^{3}$ Kansai University Faculty of Informatics, Japan

Introduction: Epigenetic factors associated with the development of autoimmune diseases are unclear. Monozygotic twin pairs discordant for positive anti-thyroglobulin autoantibodies $(\mathrm{TgAb})$ are useful to examine the epigenetic factors because of their identical genetic background. To clarify the genetic background specific for 
$\mathrm{Tg} \mathrm{Ab}$ discordant twins, we genotyped concordant and discordant twin pairs for TgAb positivity. Subjects and Methods: We selected subjects from 257 Japanese monozygotic twins, recruited from the registry established by the Center for Twin Research at Osaka University. $\mathrm{TgAb}$ positive concordant (PC) pairs were $5.7 \%$ (4 pairs) and $9.6 \%$ (18 pairs) of male and female pairs, respectively. $\mathrm{TgAb}$ discordant (DC) pairs were $11.4 \%$ (8 pairs) and $8.0 \%$ (15 pairs) of male and female pairs, respectively. TgAb negative concordant (NC) pairs were $78.6 \%$ (55 pairs) of male pairs and $74.3 \%$ (139 pairs) of female pairs. To perform stricter grouping, in this study, we set the cut-off value for positive $\mathrm{TgAb}$ to $50.0 \mathrm{IU} / \mathrm{mL}$ ( TgAb Negative $=50.0 \mathrm{IU} / \mathrm{mL}$. TgAb Borderline $\geq 28.0 \mathrm{IU} / \mathrm{mL}$ and $<50.0 \mathrm{IU} / \mathrm{mL})$. Nineteen discordant ( 6 male and 13 female pairs) and 185 concordant pairs (48 male and 137 female pairs) for $\mathrm{TgAb}$ positivity were finally examined. We genotyped gene polymorphisms using the Omni5-4 BeadChip Kit (Illumina) to clarify genetic background. Results: 155 polymorphisms specific for TgAb discordant pairs were significantly different in genotype frequencies from those of concordant pairs, and none of them was located on the HLA region of chromosome 6 , which known as susceptible to autoimmune thyroid disease. Conclusions: We found that the genetic background specific for $\mathrm{TgAb}$ discordant twins who are susceptible to epigenetic changes are different from that specific for $\mathrm{TgAb}$-positive concordant twins. (The authors are thankful to all the consultants from the Osaka Twin Research Group. This project was supported by University Grants from the Japanese Ministry of Education, Culture, Sports, Science and Technology, and by JSPS KAKENHI JP16H03261.)

\section{THE RELATIONSHIP BETWEEN LONELINESS AND SELF-REPORTS OF PHYSICAL AND MENTAL HEALTH: A CO-TWIN CONTROL STUDY}

\footnotetext{
Thomas S. Nilsen', Julia Kutschke², and Jennifer Harris'

${ }^{I}$ Norwegian Institute of Public Health, Oslo, Norway

${ }^{2}$ Medical Department, Vestfold Hospital Trust, Tønsberg, Norway
}

Introduction: Loneliness is recognised as a public health problem in Norway. Numerous studies report a strong association between loneliness with adverse health effects and increased mortality. A frequent issue is how to assess cause and effect in these relationships. We use the co-twin control model to explore the associations between loneliness with self-ratings of physical and mental health, which provides insight into the nature of the association because it controls for genetic and familial confounding. Materials and Methods: The data are based on responses from 5446 twins (1989 pairs and 1468 single responders) who participated in a questionnaire study on social factors and health. 1612 twin pairs completed the UCLA revised loneliness scale (20 items, mean score 31, mean age 60 years). Measures of self-reported physical and mental health were available for 1,950 and 1,873 twin pairs respectively. UCLA scale scores were dichotomised (cut-off at 40 points), resulting in $14 \%$ of the sample classified as lonely. The mean intrapair difference for discordant pairs was 7 (75\% had a difference score of $>10$ ). Self-rated physical and mental health were reported on 4-point scale where 'very good' and 'good' were recoded as good physical or mental health, and 'not entirely good' and 'bad' were coded as 'poor' physical or mental health; $31 \%$ and $32 \%$ reported poor physical and mental health respectively. Logistic regression was conducted and the co-twin control design was employed. Results: The $O R$ for reporting poor physical health when lonely was $2.4(95 \%$ CI $[1.9,2.9], p<.001)$ when all twins are treated as individuals. Among MZ twins discordant for loneliness, the $O R$ for reporting poor physical health was 1.4 (95\% CI [0.69, 2.9], $p=$ .4 ), and the corresponding value for DZ twins was 1.95 (95\% CI $[1.9,16.7], p<.01)$. The $O R$ for all twins treated as individuals reporting poor mental health when lonely was 6.4 (95\% CI [4.8, 8.4], $p<.001)$. Among MZ twins discordant for loneliness, the $O R$ for reporting poor mental health was $6.0(95 \% \mathrm{CI}[2.1,23.8], p<.01)$, and for DZ twins the $O R$ was 5.0 (95\% CI $[1.9,16.7], p<.01)$. Conclusions: Although confidence intervals are wide and overlapping, results point towards a causality interpretation for the association between loneliness and poor mental health. The lower $O R$ for MZ twins in the association between loneliness and poor physical health suggests partial genetic and familial confounding. These preliminary results will be followed up with further analyses.

\section{OUTCOMES OF MONOCHORIONIC DIAMNIOTIC TWIN PREGNANCIES: A COMPARISON OF ASSISTED AND SPONTANEOUS CONCEPTIONS}

Maria De La Calle, Miguel Cabrero, Eva Pena, Elena Martin Boado, Beatriz Herrero, Roberto Rodriguez, Eugenia Antolin, and Jose Luis Bartha Maternal-Fetal Unit, Obstetrics Department, University La Paz Hospital, Madrid, Spain

Introduction: As a consequence of the increase of assisted reproductive techniques (ART) there has been a rising number of monochorionic diamniotic twins (MCDA) pregnancies, which are associated with a higher number of fetal complications (FFTS, IUGR or TAPS). These complications are well known in spontaneous MCDA pregnancies but there is almost no information in ART MCDA pregnancies. Objective: We have compared the incidence of fetal and neonatal complications between spontaneous MCDA pregnancies and TRA MCDA pregnancies. Material and Methods: Case-control retrospective study. We analyzed the fetal and neonatal complications of 98 MCDA pregnancies (75 spontaneuos MCDA and 23 ART MCDA) controlled in the Maternal-Fetal Unit of Obstetric Service of Hospital La Paz, Madrid, between 2012 and 2017. Results: Of the total number of pregnancies in which IUGR appears as follows: $7.1 \%$ were type I, $2 \%$ type II, and $3 \%$ type III. In the control group (spontaneous MCDA) there were $11(14.7 \%)$ and in the group of cases (ART MCDA) 1 (4.3\%), this difference being not statistically significant $(p=.28)$. Of the total number of pregnancies in which FFTS appears, $1 \%$ were stage I, $10.2 \%$ were stage II, $4.1 \%$ were stage III and $1 \%$ were stage IV. In the control group there are 13 $(17.3 \%)$ and in the case of cases there are $3(13.0 \%)$, this difference being not statistically significant $(p=.76)$. The only case of TAPS appeared in the group of cases, with no statistically significant differences between the two groups $(p=.23)$. The neonates with weight between 1,500-2,500 g were more frequent in spontaneous MCDA group and the ones with weight $>2,500 \mathrm{~g}$ in TRA group $(O R=0.47(0.22-0.97))$. The neonates born between 32-37 weeks are more frequent in spontaneous MCBA pregnancies and the ones born $>37$ in TRA group $(O R=0.27(0,09-0,80))$. These diferencies are lost when we adjust the results by maternal age. Conclusions: ART are not related with a higher number of complications of fetuses in MCDA pregnancies. If the results are not adjusted by maternal age, there would be better outomes such as premature and neonatal weight in TRA group.

\section{THE BRAZILIAN TWIN REGISTRY: UPDATE AND BASELINE CHARACTERISTICS OF REGISTERED TWINS}

Paulo Ferreira, Guilherme Jardim, Fernando Siqueira, Vinícius Cunha Oliveira; Hércules Ribeiro Leite, and Lucas Calais Ferreira

Faculty of Health Sciences, The University of Sydney, Sydney, Australia; Programa de Pós-Graduação em Reabilitação e Desempenho Funcional, Universidade Federal dos Vales do Jequitinhonha e Mucuri, Diamantina, Brazil; Centre for Epidemiology and Biostatistics, Melbourne School of Population and Global Health, The University of Melbourne, Melbourne, Australia; CAPES Foundation, Ministry of Education, Brasilia, Brazil

Introduction: Twin studies are vital for the understanding of genetic and environmental factors on the development, prognosis, and management of health conditions. Genetic and environmental influences are likely to depend on cultural and ethnic backgrounds. The establishment of the Brazilian Twin Registry (BTR) in 2013, the first of 
its kind in South America, was therefore a promising enterprise. We here provide an update on the registry's activities and the baseline characteristics of the registered twins. Materials and Methods: More than 10,000 adult twins have been identified through a variety of sources and 839 twins have been successfully registered. An additional 300 twins under 18 years of age have been pre-registered. We collected and analysed data from 290 twins who answered the baseline questionnaire, which include sociodemographic and anthropometric variables. Data on this sample was collected between 2013 and 2017. Zygosity was determined by assessing twin similarity at baseline through the peas-in-a-pod questionnaire. Results: From the sample of 290 twins, a total of 177 twins $(61.1 \%)$ self-declared as whites, $95(32.77 \%)$ as browns, $7(2.42 \%)$ as mulattoes, $6(2.07 \%)$ as blacks, and $5(1.73 \%)$ preferred not to declare. The mean age was 32.49 years, $204(70.34 \%)$ were women and $86(29.66 \%)$ were men. A total of $5(1.72 \%)$ twins reported living less than 2 years in the same household, $4(1.38 \%)$ between 10 and 15 years, $56(19.31 \%)$ between 15 and 20 years, $125(43.1 \%)$ between 20 and 25 years, and $100(34.49 \%) 25$ years or more. The mean height was 1.65 meters (m) and the mean weight was 63.4 kilograms. The mean waist circumference was $80.08 \mathrm{~cm}$ and the hip circumference was $95.77 \mathrm{~cm}$. From zygosity determination by questionnaire Peas-in-a-Pod, 176 twins were found to be monozygotic and 40 dizygotic. There were 88 complete MZ pairs (17 male and 71 female pairs) and 20 complete DZ pairs ( 2 male, 7 female and 11 opposite-sex pairs). The BTR also includes a range of data on health conditions and lifestyle factors such as physical activity, depression, musculoskeletal pain, diabetes, and cardiovascular diseases. Conclusions: The BTR is becoming a rich resource for the international scientific community, and will help health researchers to understand human diseases and conditions with significant impact globally. Data will be available for future ethically approved studies.

\section{SMOKING AND CANCER AMONG MONOZYGOTIC DISCORDANT TWIN PAIRS: THE NORDIC TWIN STUDY OF CANCER}

Tellervo Korhonen ${ }^{1,2,3}$, Jacob Hjelmborg ${ }^{4,5}$, Klaus Holst ${ }^{6}$, Axel Skytthe ${ }^{4,5}$, Eero Pukkala $^{9,10}$, Julia Kutschke ${ }^{11}$, Jennifer R Harris ${ }^{11}$, Lorelei A Mucci ${ }^{7,8,13}$, Kaare Christensen ${ }^{4,5}$, Kamila Czene ${ }^{12}$, Hans-Olov Adami ${ }^{12,13}$, and Tuomas Scheike ${ }^{6}$

${ }^{1}$ Institute for Molecular Medicine, University of Helsinki, Helsinki, Finland ${ }^{2}$ Department of Public Health, University of Helsinki, Helsinki, Finland ${ }^{3}$ Institute of Public Health and Clinical Nutrition, University of Eastern Finland, Kuopio, Finland

${ }^{4}$ Department of Epidemiology, Biostatistics and Biodemography, University of Southern Denmark, Odense, Denmark

${ }^{5}$ The Danish Twin Registry, University of Southern Denmark, Odense, Denmark ${ }^{6}$ Department of Biostatistics, University of Copenhagen, Copenhagen, Denmark ${ }^{7}$ Channing Division of Network Medicine, Brigham and Women's Hospital, Harvard Medical School, Boston MA, USA

${ }^{8}$ Centre for Public Health Sciences, University of Iceland, Reykjavik, Iceland ${ }^{9}$ Finnish Cancer Registry, Institute for Statistical and Epidemiological Cancer Research, Helsinki, Finland

${ }^{10}$ School of Health Sciences, University of Tampere, Tampere, Finland

${ }^{11}$ Division of Epidemiology, The Norwegian Institute of Public Health, Oslo, Norway

${ }^{12}$ Department of Medical Epidemiology and Biostatistics, Karolinska Institutet,

Stockholm, Sweden

${ }^{13}$ Department of Epidemiology, Harvard School of Public Health, Boston, MA, USA

Introduction: There is strong evidence of the role of smoking in cancer. However, analysis of discordant monozygotic (MZ) twin pairs is one of the most powerful designs to investigate causal nature of such association. We investigated the associations of smoking with selected cancer types while controlling for genetic and shared environmental confounding. Material and Methods: NorTwinCan includes population-based cohorts from the Danish, Finnish, Norwegian, and Swedish twin registries. Each twin has an individually unique national registration number, allowing for linkage to the national cancer and mortality registries with complete follow-up, drop-out being only due to death or emigration. Cancer occurrence was obtained from the national cancer registries and computed from the baseline when smoking status was determined until the end of follow-up. We classified the participants as never, former or current smokers. We focused on MZ twin pairs where one co-twin had any of the following histologically confirmed cancer (Bladder, Kidney, Larynx, Liver, Lung, Oral cavity Oesophagus, Pancreas) while his/her co-twin had no cancer. Results: We recorded total of 988 incident cancers among never smokers, 2394 cases in current and 630 cases in former smokers. Time-to-event analysis based on individuals showed that in comparison to never smokers, current and former smokers had elevated hazard ratios $(\mathrm{HR})=3.06$ (95\% CI $[2.47$, $3.79])$ and $\mathrm{HR}=1.77$ (95\% CI [1.40, 2.25]) respectively. Among the MZ twin pairs which were discordant for cancer status (one twin had while his/her co-twin did not have cancer), the currently smoking co-twin had HR=2.66 (95\% CI $[1.72,4.11])$ and for the formerly smoking co-twin $\mathrm{HR}=1.87$ (95\% CI $[1.16,3.03]$ ) in comparison to never-smoking co-twin. Conclusions: The associations of smoking with several types of cancer found in individuals were replicated among the discordant identical twin pairs.Our data confirm that the association of smoking with cancer is not confounded by shared genetic or environmental influences.

\section{SUCCESSFUL CERVICAL CERCLAGE IN MULTIPLE PREGNANCY ACCORDING TO A CLINICAL CASE}

N. Abián Franco, B. Gastón Moreno, S. García Francés, M. Ruiz García, I. Zabaleta Loinaz, B. Pérez Munárriz, L. Martinez Jimenez, A. Safont Gascón, I. Jimenez Ubieto, M. Huarte Ciganda, C. Larrañaga Azcárate, and J. Zabaleta Jurío Obsterics and Gynaecology, Complejo Hospitalario de Navarra, Spain

Clinical Case: A 32-year-old (gravida 3 para 3), came asymptomatic to the first antenatal visit. She was at 9th weeks of spontaneous bichorionic-biamniotic gestation. As relevant obstetrical background it must be pointed out her first pregnancy that ended in preterm delivery at 35 weeks of pregnancy seven years ago. During her second pregnancy delivered at term five year ago, she presented painless cervical dilatation (amniotic sac prolapsed in vagina) in 15th week of pregnancy and urgent Shirodkar cervical cerclage was performed. It was scheduled a prophylactic Shirodkar cervical cerclage in 13rd week of this current gestation, with no incidents recorded. The cerclage was removed electively at 36 weeks of gestation. During third trimester, fist fetus remained in breech presentation. Fetal wellbeing controls were performed, and expectant management was chosen until 38 weeks of gestation. During the admission for practicing cesarean section due to first fetus breech presentation at $38+3$ weeks, it was objectified spontaneous change to cephalic presentation of first fetus. Owing to favorable Bishop test, it was decided to start induction of labor with endovenous oxytocin under continuous fetal cardiotocography control. First fetus eutocic delivery occurred after 8 hours of dilatation period, and the first newborn weight was 2,515 grams. The second fetus was delivered manually, assisted on account of traverse fetal lie, and weight for this second newborn was 2,360. Uterine involution in immediate postpartum occurred but blood loss was quantified higher than usual for vaginal delivery. First fetus umbilical cord $\mathrm{pH}$ was 7.27 and 7.21 for the second fetus. Apgar score for first fetus was 9 during the first minute and 10 for the first five minutes of life. The second fetus presented an Apgar score 7 during the first minute and 9 for the first five minutes of life. Both newborns were admitted to the maternal department and no intensive care were needed. Our patient needed one month of oral ferro therapy for mild anemia, and she recovered normally during the postpartum period. Conclusions: From our experience, we empirically support urgent and prophylactic cervical cerclage in good candidates with twin pregnancies, despite lack of wide scientific support. Nevertheless, further studies are necessary in order to conclude a positive risk-benefit balance in this respect. 


\section{TWIN-TWIN TRANSFUSION SYNDROME: ABOUT A CASE} REPORT

I. Zabaleta, M. Ruiz, B. Gastón, J. Barrenetxea, N. Abian, A. Safont, I. Pedroarena, M. Bazán, M. Urtasun, S. Garcia, I. Jimenez, and L. Martinez

Obstetrics and Gynecology Service, Hospital Center of Navarra, Pamplona, Spain

Introduction: Twin-to-twin transfusion syndrome (TTTS) is one of the most important complications of monochorionic multiple gestation due to high risk of fetal and neonatal mortality. The physiopathology of this complication is not already clear. We know that in monochorionic gestations there are placental intertwin vascular anatomoses and these play a very important role in this process. The precipitating event for the development of TTTS is likely to be relative hypovolemia in one twin (called the donor twin) because of the imbalance in placental vascular anastomoses. This process produce oliguria in the donor twin thar finally results in anhydramnios and the 'stuck twin' phenotype. Changes in the donor leads to hypervolemia in the non-donor twin (called the recipient twin) and polyuria and polyhydramnios finally occurs in the recipient twin, appearing as cardiovascular changes. TTTS is suspected by ultrasound examination and evidence of monochorionic placentation with discordant amniotic fluid volumes (maximum vertical pocket: donor $80 \mathrm{~mm}$ ). To classify the TTS syndrome there are lots of classification systems. One of the most used system is Quintero stages classification, based on Doppler velocimetry in the umbilical artery and vein and ductus venosus, and in the visualization of the bladder. The clinical evidence shows that we should monitor monochorionic pregnancies for development of TTTS with serial ultrasound examinations. When TTTS appears, the management is different according to the stage of the syndrome. The managment options are expectant management, fetoscopic laser ablation of the placental anastomosis, or amnioreduction. Case Report: This is a case of a 36-year-old woman with secondary infertility history. She got pregnant by in vitro fertilization. On the 12th week of pregnancy a monochorinic biamniotic twin gestation was diagnosed. On the 14th week of gestation TTTS signs appeared but at the 16th week those signs disappeared. On the $18+2$ week signs of STFF again appeared at stage II, so fetoscopic laser ablation of the placental anastomosis was offered to the couple. No new TTTS signs and no other complications derived from the treatment appeared. Conclusions: TTTS is a very important complication of monochorionic gestations. It is necessary to monitor these pregnancies to detect this process. When TTTS is established, a correct classification and an indicated treatment are necessary.

\section{ACCURACY OF A ZYGOSITY DETERMINATION QUESTIONNAIRE OF ADULT TWINS AND TWIN CHILDREN}

Tessa L. Cutler, Katrina Scurrah, and John L . Hopper

Twins Research Australia, Melbourne School of Population and Global Health, The University of Melbourne, Melbourne, Australia

Introduction: Accurate determination of zygosity is critical for twin research; however, it is not always feasible to conduct DNA zygosity testing for all participants. Twin registries and studies often rely on self-reported zygosity or use questionnaires to determine zygosity. This project aimed to test the accuracy of a simple, three question zygosity determination questionnaire. Methods: Zygosity determination questionnaires were included in a questionnaire sent to Twins Research Australia members. The questionnaire was completed by adult twins and parents of twin child. These members provided opinions and information about their zygosity from themselves, parents and doctors as well as available DNA zygosity test results. Individual responses to the determination questionnaire were compared with paired responses to determine accuracy of single twin responses. Both individual and paired responses were compared with DNA zygosity test results. Self-reported, parent-reported and doctor-reported zygosity was compared with DNA zygosity test results. Results: DNA results were obtained for 857 adult twin pairs where at least one twin had completed the zygosity questionnaire (780 pairs MZ and 77 pairs DZ; 442 pairs where both completed zygosity determination questionnaire and 411 pairs where only one twin completed the questionnaire). DNA results were obtained for 860 twin pairs under 18 years of age where a parent completed the zygosity questionnaire about the twin children (708 pairs MZ and 152 pairs DZ). Of the adult responses, $97 \%$ of questionnaires completed by both twins returned a correct result (100\% of MZ and 52\% of DZ) while $96 \%$ of questionnaires results by individual twins returned a correct result. Of the child responses, $90 \%$ of questionnaires completed by a parent about both twins returned a correct result (94\% of MZ and 68\% of DZ). Conclusions: The short zygosity determination questionnaire provided accurate zygosity of MZ twins with reduced accuracy for DZ twins. Accuracy was higher for children compared with adults and for adults the accuracy was slightly higher where both twins completed the questionnaire compared with only one twin completing the questionnaire. Results: for MZ pairs were significantly more accurate than results for DZ pairs and further validations should be conducted with larger samples of DZ twins.

\section{EPIGENETIC STUDIES OF TWINS: INSIGHTS INTO NONSHARED ENVIRONMENT}

Jeffrey M. Craig', Richard Saffery', Yuk Jing Loke', Anna Czajko', Boris Novakovic', Eric J. Joo', Alicia Oshlack', Jovana Maksimovic ${ }^{\prime}, K^{\prime}$ atrina Williams', David Amor' ', Kylie Crompton' ', Dinah Reddihough' ', Sue Reid' , Lata Vadlamudi Michael Permezel ${ }^{3}$, Euan Wallace ${ }^{4}$, and Mark Umstad ${ }^{5}$

${ }^{I}$ Murdoch Childrens Research Institute, Royal Children's Hospital, Parkville, Melbourne

${ }^{2}$ Faculty of Medicine, University of Queensland Centre for Clinical Research, Queensland, Australia

${ }^{3}$ The Ritchie Centre, Monash Institute of Medical Research, Victoria, Australia ${ }^{4}$ Department of Obstetrics and Gynaecology, University of Melbourne, Mercy Hospital for Women, Heidelberg, Victoria, Australia

${ }_{5}^{5}$ Department of Obstetrics and Gynaecology, University of Melbourne, Royal Women's Hospital, Melbourne, Victoria, Australia

Introduction: The Peri/postnatal Epigenetic Twins Study (PETS) is a unique cohort of 250 mothers and their twins. Women were recruited from three Melbourne hospitals midway through their second trimester, which enabled measurement of maternal and fetal factors at multiple time points. We collected multiple biospecimens at birth (cord blood, cords, placenta and buccal tissue) and repeat samples of blood and buccal swabs when infants were 18 months and 6 years of age. We aimed to study the plasticity of epigenetic marks and the genes they control during the intrauterine period and in early childhood. The UNIQUE twin study is a program of research exploring the epigenetic mechanisms and biomarkers of neurodevelopmental disorders using discordant MZ co-twin models. We have cohorts of 11-16 twin pairs discordant for cerebral palsy, autism or epilepsy, and are commencing a study of developmental coordination disorder (dyspraxia). Materials and Meth$o d s$ : We performed genome-wide analysis of the epigenetic mark of DNA methylation in multiple cell types using Illumina Infinium arrays. Within-pair DNA methylation state was calculated across twin pairs with respect to birth weight or disease state. Results: Twin pairs exhibited a wide range of within-pair epigenetic discordance at birth in genes associated with development and response to environment. Genes whose expression and methylation levels correlated with birth weight in $\mathrm{MZ}$ pairs were enriched in functions related to cardiometabolic function and lipid metabolism. We have also identified a number of regions whose DNA methylation state in DNA from neonatal dried blood spots correlates with a future diagnosis of cerebral palsy. Conclusions: Our data support the idea that nonshared (feto-placental) environmental factors have the greatest impact on the developing epigenome. They also suggest that multiple early environments may be epigenetically reprogramming genes 
involved in metabolism, which may provide a mechanism for the early origins of cardiometabolic disease. Data from CP-discordant $\mathrm{MZ}$ wins suggests the potential for predictive epigenetic biomarkers for the disorder.

\section{GROWTH AND BODY COMPOSITION OF TWINS PREMATURE INFANTS ACCORDING TO GESTATIONAL AGE AT BIRTH}

Nadia Liotto, Paola Roggero, Pasqua Piemontese, Anna Orsi, Orsola Amato, Daniela Morniroli, Maria Lorella Giannì, and Fabio Mosca

Fondazione IRCCS Ca Granda Ospedale Maggiore Policlinico, Neonatal Intensive Care Unit, Department of Clinical Science and Community Health, University of Milan, Italy

Introduction: Twin infants can show an altered fetal and postnatal growth compare to singletons. Poor data are available on growth and body composition of twins preterm infants. Aim of the study was to compare growth and body composition of twins and singletons premature infants from birth to term corrected age. Materials and methods: Anthropometric measurements — weight $(\mathrm{g})$, length $(\mathrm{cm})$ and head circumference $(\mathrm{HC})(\mathrm{cm})$ - were performed at birth and at term corrected age (CA) on 572 infants (221 twins). Body composition was assessed at term CA in all infants enrolled by an air-displacement plethysmography. Infants were categorized as extremely preterm infants (EPI: GA $<28$ weeks), very preterm infants (VPI: GA from $281 / 7$ to 32 weeks), moderate preterm infants (MPI: GA from $321 / 7$ to 34 weeks) and late preterm infants (LPI: GA to $341 / 7$ to 36 weeks). Results: Infants enrolled were: EPI: 11 twins and 32 singletons, VPI: 60 twins and 107 twins, MPI: 29 twins and 46 singletons, LPI: 121 twins and 166 singletons. Overall infants 279 (48.8\%) were male. Anthropometric measurements at birth and at term CA were similar between MPI, VPI and EPI twins compare to singletons. Furthemore, no differences were found in fat mass and fat-free mass amount in these category of infants: Fat mass (g): EPI twins $480 \pm 169$ versus EPI singletons $537 \pm 225$, VPI twins $508 \pm 198$ versus VPI singletons $481 \pm 183$, MPI twins $416 \pm 171$ versus MPI singletons $371 \pm 163$; Fat free mass (g): EPI twins $2354 \pm 480$ versus EPI singletons $2375 \pm 470$, VPI twins $2418 \pm 350$ versus VPI singletons $2352 \pm 438$, MPI twins $2201 \pm 413$ versus MPI singletons $2140 \pm 358$. LPI twins showed a lower weight and length at birth and at term CA compare to singletons $(2274 \pm 358 \mathrm{~g}$ and $44.7 \pm 2.4$ vs. $2496 \pm 400$ and $45.5 \pm 2.4$ respectively at birth, $3273 \pm 475$ and $48.8 \pm 2.1$ vs $3468 \pm 523$ and $49.8 \pm 2.5$ respectively at term CA; $p<.002)$. Fat free mass $(\mathrm{g})$ was also lower in these infants compare to singletons $(2783 \pm 362 \mathrm{~g}$ vs. $2932 \pm 412$ respectively). Conclusion: At term CA, moderate, VPI and EPI twins showed growth and body composition similar to singletons whereas LPI twins showed a different growth pattern compare to singletons, characterized by a fat-free mass deficit. Further study is necessary to explore growth and body composition later in life.

\section{TWINNING AND MULTIPLE BIRTH RATES ACCORDING TO MATERNAL AGE IN THE ENTIRE COUNTRY OF BRAZIL AND ITS FIVE REGIONS FROM 2003 TO 2014}

Marco Antonio Corrêa Varella ', Tiziana G. Acquaviva' , Eloisa De Souza

Fernandes', Tania Kiehl Lucci', Jonas Arantes ${ }^{2}$, Sylvia Corte ${ }^{3}$, Noelia Gomes ${ }^{3}$,

Vinicius Frayze David', Nancy L. Segal ${ }^{4}$, Vera Silvia Raad Bussab', Fernando Leite

Ribeiro', Jaroslava Varella Valentova', and Emma Otta'

${ }^{1}$ Department of Experimental Psychology, Institute of Psychology, Universidade de São Paulo, São Paulo, Brazil

${ }^{2}$ Institute of Biosciences, Universidade de São Paulo, São Paulo, Brazil

${ }^{3}$ Faculty of Sciences, Universidad de La Republica, Montevideo, Uruguay

${ }^{4}$ Department of Psychology, California State University, Fullerton, USA

Brazil is a populous country that is culturally, ethnically and economically diverse, so it presents many opportunities for twin research. In contrast to more developed countries, however, there is still a gap regarding country-wide twinning rates, since current studies have focused on a city or a few hospitals within a city. The USP Twin Panel research team filled this gap by exploring twinning rates for the entire country and its five regions between 2003 and 2014, and its relationship with maternal age. The data were drawn from an official public database and transformed into rates of maternities. We performed correlations between time period and maternity rates separately for the five Brazilian regions: South, Southeast, CentralWest, North, and Northeast. We performed correlations separately for eight categories of mothers' age (45 years). A multivariate general linear model (GLM) was used to explore the effects of region, maternal age, and time period on singleton, twin and higher order multiple birth rates. The overall average rates of singletons, twins, and higher order multiple births per 1,000 maternities (\%) were $987.34,10.36$, and 0.66 , respectively. In general, the developed regions (e.g., Southeast) presented higher twinning and multiple birth rates than underdeveloped ones (e.g., Northeast). Our GLM results showed that maternal age strongly positively predicted twin (partial $\eta^{2}=0.56$ ) and multiple (partial $\eta^{2}=0.21$ ) birth rates, and negatively predicted singleton birth rates (partial $\eta^{2}=0.62$ ). The decade time period predicted also positively, albeit weakly, twin birth rates (partial $\eta^{2}=0.03$ ), negatively predicted singleton rates (partial $\eta^{2}$ $=0.02$ ), and had no effect on multiple birth rates. North and NorthEast regions had the lowest rates of twins relative to the other regions ( $p$ s <.001), and South, South-East and Central-West regions did not differ from each other in the twin rates. The South-East had larger rates of multiple births than the other regions $(p s<.001)$. The observed pattern of results from this country-wide decade-long study is consistent with the literature: it points to the existence of an agedependent mechanism that leads to a strong increase in twinning and multiple births, at the expense of singleton births, among older mothers. Further studies should explore how local socioeconomic factors influence between-regions difference in twinning rates.

\section{PULMONAR ACUTE EDEMA IN A MONOCHORIONIC TWIN PREGNANCY COMPLICATED WITH THE DEATH OF ONE} TWIN

María Huarte Ciganda, Izarbe Jiménez Ubieto, Lara Martinez Jiménez, Beatriz Pérez Munárriz, Anabel Ochoa Prat, and Blanca Bezares Pérez Complejo Hospitalario Navarra B, Pamplona, Navarra, Spain

Introduction: All twin pregnancies are at increased risk of preterm delivery, congenital anomalies, and growth restriction compared with singleton pregnancies. Case Report: A 33-year-old woman had an spontaneous monochorionic diamniotic gestation. Pregnancy was uneventful until the 27th week of gestation. Abdominal ultrasound scans were made every 2 weeks to exclude complications. At 28 $4 / 7$ weeks she was admitted for vaginal bleeding and contractions. Shortening of the cervical length $(22 \mathrm{~mm})$ was found. Death of the first twin was confirmed during the abdominal scan. The fetus had signs of cardiac failure. An hidramnios was detected in the second amniotic sac. A twin-to-twin transfusion syndrome was suspected. Antenatal corticosteroids, magnesium sulfate for fetal neuroprofilaxis and tocolytic treatment (atosiban) was started. During her admission to hospital, the patient suddenly developed dyspnea at 29 1/7. A low oxigen saturation was detected (89\%). The thorax $\mathrm{X}$-ray showed a small pleural effusion in left lung. An ecocardiography showed normal changes related to pregnancy. To exclude a pulmonar tromboembolism a computerised tomography pulmonary angiogram was done, which was normal. An acute pulmonar edema was suspected. The patiet required admission to an intensive care unit during pregnancy. The patient improved after various courses of furosemida and supplemental oxygen. Maintenance tocolytic with atosiban was administered from 29 5/7 weeks of pregnancy because the patient had uterine contractions and a shortened cervix. At $306 / 7$ magnesium sulfate for neuroprotection was restarted and a repeat course of maternal corticosteroid was given because the patient went 
into labor. A cesarean section was done at 31 weeks of pregnancy. A dead fetus $(960 \mathrm{~g})$ was delivered. The alive co-twin weighed 1,360 g. Conclusions: Acute pulmonary edema occurs in approximately $0.08 \%$ of pregnancies. Approximately $50 \%$ of the cases of pulmonary edema were attributed to tocolytic therapy or cardiac disease. The remaining cases were ascribed to either preeclampsia or iatrogenic volume overload. Preterm labor increases the risk of pulmonary edema, likely due to the increased exposure of these patients to tocolytic therapy

\section{WELLBEING AND THE BRAIN}

Meike Bartels, Bart Baselmans, Rick Jansen, HillFung Ip, Jenny van Dongen, Abdel Abdellaoui, Margot van de Weijer, Gonneke Willemsen, Jouke Jan Hottenga, Eco de Geus, Dorret Boomsma, and Michel Nivard

Department of Biological Psychology, Vrije Universiteit Amsterdam, the Netherlands

Introduction: Wellbeing plays an important role in psychology and medicine, as well as in economics. Wellbeing owes its interdisciplinary prominence to its associations with physical and mental health, and its role as a desired socio-economic outcome and index of economic development (Helliwell et al., 2015). In our recent work, we identified many genetic variants that are associated with wellbeing. Material and Methods: In order to more accurately pinpoint brain regions where genes relevant to the wellbeing spectrum are differentially expressed, we computed stratified LD scores based on differential gene expression in an anatomically comprehensive set of 210 brain regions, based on 3,707 measurement in 6 human brains (Hawrylycz et al., 2012). For each brain region, genes were selected that showed higher expression compared to all other regions, inclusion is determined based on a $t$ statistic of the difference test. For each region, we selected the top $10 \%$ most strongly expressed genes. Results: The LD scores were significantly enriched at FDR $<0.05$ at multiple gyri in the cortex (e.g., fusiform gyrus, parahippocampal gyrus and precentral gyrus) as well as the precuneus, planum polare, temporal pole and the superior -and paracentral lobule. To reveal regions related to the wellbeing spectrum within these regions, we computed differential brain expression only within the cortex, subcortical structures, brainstem and cerebellum and identified enrichment of genes specifically expressed in the subiculum $(z=3.60, p<.001)$. The subiculum is considered part of the hippocampal formation and plays a key role in hippocampal-cortical interaction (O'Mara et al., 2001) in the inhibition of the hypothalamic-pituitary-adrenal-axis and the human response to stress (Herman \& Mueller, 2006). Conclusions: By leveraging the genome-wide results, LD score regression, and an atlas of brain gene expression, we were able to pinpoint brain regions where region specific gene expression exists for genes enriched in their effect on wellbeing, and we report evidence for enrichment of genes differentially expressed in several cortical regions, as well as in the subiculum.

\section{SOME ASPECTS OF PARENTAL SUPPORT FOR TWINS' EDUCATION}

Kristiina Heinonen' and Jaakko Kaprio ${ }^{2}$

${ }^{I}$ Department of Nursing Science, University of Eastern Finland, Savo Vocational College, Kuopio, Finland, Department of Public Health, University of Helsinki, Finland ${ }^{2}$ University of Helsinki, Helsinki, Finland

Introduction: A previous study on the learning disabilities of twins found that the 'Generalist Genes Hypothesis' held for language and general cognitive disabilities, as well as reading and mathematics disabilities. There was also a need to improve consultation between families and schools and to make important information more widely available to parents. Our study aims to provide a perspective on cooperation between families and the school, and to uncover kinds of learning difficulties experienced by twins at school. Materials and Methods: The study sample consisted of responses from par- ents of 263 twin pairs born 1983-1984 taking part in the FinnTwin12 study. Comparisons focused on gender differences (boys vs. girls) and birth order (A vs. B twins). The data were obtained through interviewing with a questionnaire. The key themes are the individuality of twins, cooperation between parents and the school and the support needed by twins in their schoolwork. Content analysis and NVivo was used. Results: The parents' decision to put their children in the same class was often compelled by circumstances. Being close together was felt to be easier and safer for the children themselves. As the children grew, twins also experienced being in a different class. The decision to have them in different classes was influenced by a child's need for special support, promotion of their independence and behavioral problems. Parents contacted the teacher about learning difficulties, cases of bullying, issues related to a child's activeness and disturbance, special support needs and health problems. The school also contacted families about problems related to understanding, sloppiness and reading problems. Twins had learningrelated problems especially in mathematical subjects, languages and theoretical subjects. Parents supported the twins' school progress by motivating, encouraging and listening and going over things at home. Parents reported emphasizing the similarity of the children, particularly with similar clothes when the children were small. In steering children towards hobbies, both taking account of their individuality and offering the same hobby were reported. Conclusions: More attention should be given to a twin's learning process and support measures, particularly in mathematics, languages and theoretical subjects. Learning-related problems appear in different sets of twins and the need for support in the learning process with twins is more parallel than different.

\section{FAMILIAL RISK OF CROSS-CANCER ASSOCIATIONS: FINDINGS FROM THE NORDIC TWIN STUDY OF CANCER (NORTWINCAN)}

Jennifer R. Harris ${ }^{1}$, Jaakko Kaprio ${ }^{2}$, Kamila Czene $^{3}$, Lorelei Mucci ${ }^{4}$, Eero Pukkala ${ }^{5}$, Kaare Christensen ${ }^{6,7}$, Thomas Scheike 8; Hans-Olav Adami ${ }^{3,9}$, and Jacob Hjelmborg 6,7

${ }^{1}$ Department of Genetic Research and Bioinformatics, The Norwegian Institute of Public Health, Oslo, Norway

${ }^{2}$ Institute for Molecular Medicine, University of Helsinki, Helsinki, Finland ${ }^{3}$ Department of Medical Epidemiology and Biostatistics, Karolinska Institutet, Stockholm, Sweden

${ }^{4}$ Channing Division of Network Medicine, Brigham and Women's Hospital, Harvard Medical School, Boston, USA

${ }^{5}$ Finnish Cancer Registry, Helsinki, Finland; 6. Department of Epidemiology, Biostatistics and Biodemography, University of Southern Denmark, Odense, Denmark ${ }^{7}$ The Danish Twin Registry, University of Southern Denmark, Odense, Denmark ${ }^{8}$ Department of Biostatistics, University of Copenhagen, Copenhagen, Denmark ${ }^{9}$ Institute of Health and Society, University of Oslo, Oslo, Norway

Introduction: NorTwinCan, based on the Nordic cancer and twin registries, comprises the worlds' most comprehensive database of twins for studying cancer. Our previous analyses of NorTwinCan data explored familial clustering for 40 types of cancer and revealed that twin concordance for cancer manifests most often across, rather than within anatomical sites. Among the cancer-concordant pairs, $62 \%$ (861 pairs) of the $\mathrm{MZ}$ and $74 \%$ (1,437 pairs) of the DZ pairs were discordant for the site of cancer. We explored familial risk underlying these cross-cancer associations. Materials and Method: We studied cross-cancer associations using the following analyses that take into account censoring and competing risk of death: (a) cross-cancer concordances that represent the lifetime risk that a pair will develop a particular combination of cancers, (b) cross-casewise concordance that estimates the risk to one twin for a cancer type given that their co-twin has a different cancer, (c) relative recurrence risks, which provide information about how likely the particular combination of cancer-co-occurrences is in the pair compared to non-related individuals, (d) estimates of heritable effects that reflect the importance of genetic influences for explaining specific sets of cross-cancer associations, and (e) time to event analyses revealing 
the average within pair difference between cancer occurrences. $R e$ sults: Our analyses revealed a great many linkages between sets of cancers. The relative recurrence risk (RR) among MZ and DZ pairs is often significantly greater than among unrelated individuals, and the RR_MZ is significantly elevated above the DZ value for many of these cross-cancer occurrences. Time to event analyses also revealed that median differences in age at diagnosis were less among MZ than DZ pairs for certain sets of cancer. Conclusions: Although the specific pattern of effects varies across the different sets of associations, familial factors underlie most of the cancer co-occurrences within pairs, and these are often explained by genetic factors. Evidence also suggests genetic effects are important for the timing of disease development for certain sets of cancers.

\section{OBSTETRIC AND NEONATAL COMPLICATIONS IN TWIN PREGNANCIES AFTER OOCYTE DONATION}

Félix Boria, Miguel Cabrero, Alejandra Mateos, María Martín-Cameán, and María De La Calle

Obstetrics And Gynecology Department, University Hospital La Paz, Madrid, Spain

Introduction: During the past years, oocyte donation (OD) pregnancies have been dramatically increased. The literature suggests that among in vitro fertilization (IVF) users, women using OD are at a higher risk of obstetrical complications than women who use their own oocytes (AO). Most of the studies include only singleton pregnancies. As multiple pregnancies are frequent among IVF users, and twinning is an independent risk factor for many obstetric and neonatal complications, we consider that a study comparing only twin populations would help us to distinguish the separate roles of OD conception and twinning in perinatal complications. Materials and Methods: We conducted an observational single-center retrospective study that included 100 women with twin pregnancies who gave birth after 24 weeks of gestation from January 1,2012 , to December 31, 2016 (50 oocyte donation pregnancies and 50 autologous oocytes) in La Paz Hospital. We reviewed each woman's complete obstetrical medical file. Women were classified into two groups: IVF with OD (OD) and IVF with AO (AO). We collected from the obstetrical records the mother's social and demographic characteristics and obstetrical histories, obstetrical complications, gestational age at birth, mode of delivery, and neonatal complications. Perinatal outcomes studied were: pregnancy induced hypertension, preeclampsia, gestational diabetes, cholestasis of pregnancy, anemia, premature rupture of the membranes, cesarean delivery, preterm birth, threatened preterm labor, birth weight, apgar score and fetal acidemia. For qualitative variables, the groups were compared with the use of chi-square test or Fisher exact test, as appropriate. For quantitative variables, we used Mann-Whitney U-test, as appropriate. The statistical analyses were performed with the use of SPSS 20. Results: There was an increased incidence of preeclampsia (OD 24\%, AO $8 \%, p=.029$ )and preterm birth (OD $52 \%, \mathrm{AO} 32 \%, p=.043$ ) in the OD group compared with the AO. The cesarean delivery rate was also higher in the OD group than in the AO (OD 90\%, AO 33\%, $p=.004)$. The OD group was not at higher risk than the $\mathrm{AO}$ for other complications. Conclusions: $\mathrm{OD}$ twin pregnancies are associated with a higher risk of preterm birth and incidence of preeclampsia than $\mathrm{AO}$ twin pregnancies.

\section{RECIDIVIST PRETERM DELIVERY IN A WOMAN WITH MULTIPLE RISK FACTORS AND CURRENT MONOCORIAL BIAMNIOTIC PREGNANCY: A CASE REPORT}

María Cabanes, Miguel Cabrero, María Martín, Alejandra Mateos, María De La Calle, and José Luis Bartha

Obstetric Dept, Hospital Universitario La Paz, Madrid, España

Introduction: Preterm birth refers to a delivery that occurs before 37 weeks of gestation. According to the gestational age, it might be moderate-to-late preterm delivery ( 32 to $<37$ weeks), very preterm delivery ( 28 to $<32$ weeks) or extremely preterm delivery $(<28$ weeks). There are many factors related to this complication of pregnancy. History of preterm birth is the major risk factor for recurrence, especially when it occurrs at 23 to 27 weeks. Other risk factors are multiple gestation, uterine malformations, short cervical length and dilated cervix, systemic infection and bacteriuria or excessive uterine contractility. Intervention to prevent preterm delivery depends on the cause: progesterone supplementation for short cervical length or previous history of preterm delivery, surgery for uterine malformations, cerclage for short cervical length or cervical insufficiency, the controversial use of progesterone and pessaries in multifetal gestation or treatment for asymptomatic bacteriuria. Case Report: We report a case of recidivist preterm delivery in a monocorial biamniotic pregnancy. A 31-year-old woman attended our High Risk Obstetrical Services after spontaneus monocorial biamniotic 7 weeks' pregnancy. She had been diagnosed with bicornuate uterus before her previous single pregnancy and being followed as a high risk patient, which ended up in a preterm birth at the 26th week of pregnancy despite the tocolytic efforts. Two resections of the septum were performed by hysteroscopic surgery after her first preterm delivery to prevent new episodes in future pregnancies. During her twin pregnancy, progesterone was offered as a way to prevent a new preterm delivery, as well as a prophylactic cerclage at the 13th week to prevent cervical insufficiency. However, threatened preterm labor occurred at the 25th week of pregnancy, but able to be stopped until the 30th week due to early management and early tocolysis, with a better neonatal result. Conclusions: There are many risk factors for preterm labor and delivery. Previous history of preterm labor is the strongest risk factor, and it normally occurs at the same gestational age. It is important to identify high risk patients to prevent reversible risk factors and lead to interventions that might be helpful to prevent for this complication in order to have better neonatal results.

\section{ZYGOSITY, ANEUPLOIDY, AND GENDER COUNT IN TWIN GESTATIONS USING SNP-BASED NIPT}

Samantha Leonard, Sheetal Parmar, Gabriel McNeill, Akshita Kalyan, Elizabeth Rivers, Ling Meng, Phikhanh Vu, and Allison Ryan

Natera Inc., San Carlos, California, USA

Introduction: Non-invasive prenatal testing (NIPT) for fetal aneuploidy using cell-free DNA in maternal plasma now routinely identifies high-risk gestations at $\geq 9$ weeks gestational age (GA). Monozygotic (MZ) twin gestations that are monochorionic are at an increased risk of fetal loss associated with twin-to-twin transfusion syndrome, however, NIPT has previously been unable to accurately report zygosity in twin gestations. Here, we report the performance of a single-nucleotide polymorphism (SNP) based NIPT to determine zygosity, presence of aneuploidy, and individual fetal gender in twin gestations. Materials and Methods: Blood was collected from women at $\geq 9$ weeks GA with twin pregnancies; clinical truth on zygosity, chromosome copy number, and gender count were collected. Plasma was isolated and analyzed for zygosity, chromosome copy number at chromosomes 21,18 , and 13 , and gender count (two males $[\mathrm{MM}]$, one male and one female $[\mathrm{MF}]$, or two females $[\mathrm{FF}]$ ) and compared to clinical truth. Sensitivity and specificity were reported, and no-call rates were estimated using a weighted average based on population prevalence of zygosity (1/3 MZ and 2/3 dizygotic [DZ]). 95\% confidence intervals (CI) were calculated using the MOVER method. Results: A total of 137 twin gestations were examined. Zygosity accuracy was $100 \%$ (MZ: 32/32; 95\% CI [89.1, 100], DZ: 61/61; 95\% CI [94.1, 100]). The estimated no-call rate for the zygosity test was $4.1 \%(4 / 97 ; 95 \%$ CI $[1.5,11.2])$. Aneuploidy sensitivity was $100 \%$ (11/11; MZ: one trisomy 21 ; DZ: four trisomy 21 , five trisomy 18 , one trisomy 13]; CI: 69.2-100) as was specificity (80/80; MZ: 22; DZ: 58; 95\% CI [84.6, 100]). Eleven samples did not receive a result for aneuploidy (two DZ trisomy 21 , nine 
DZ euploidy) with an estimated aneuploidy no-call rate of $10.8 \%$ $\left(\mathrm{MZ}:[1 / 3] *[0 / 21]+\mathrm{DZ}:[2 / 3]^{*}[11 / 68]\right) ; 95 \%$ CI $\left.[5.6,19.8]\right)$. Gender for each fetus was called with $100 \%$ accuracy $(105 / 105,95 \%$ CI [83.9, 100]; 41 MM, 34 MF, 30 FF; MZ: 43, DZ: 62). One sample did not receive a result for gender (DZ), with an estimated nocall rate of $1.1 \%$ (MZ: [1/3]*[0/43] + DZ: [2/3]*[1/63]); 95\% CI $[0.027,6.4])$. Conclusions: This study validates the ability of this SNP-based NIPT to accurately detect aneuploidy, and is the first study to accurately call zygosity and gender of each fetus, in twin gestations.

\section{TRAP SYNDROME: AN ATYPICAL CASE REPORT \\ André Cunha', Jorge Telles', Raquel Tomaz', Mariana Lupchinski', Cristine Dietrich', Osvaldo Artigalás', Daiane Schneider ${ }^{2}$, and Rafael Rosa ${ }^{3}$ \\ ${ }^{I}$ Hospital Materno Infantil Presidente Vargas, Porto Alegre, Brazil \\ ${ }^{2}$ Hospital de Clínicas, Porto Alegre, Brazil \\ ${ }^{3}$ Universidade Federal de Ciências da Saúde de Porto Alegre, Porto Alegre, Brazil}

Introduction: Reversal arterial transfusion syndrome (TRAP) affects 1: 35,000 pregnancies and 1\% of monozygotic pregnancies, and is characterized by inversion in the umbilical artery flow of one of the twins. When the insertions of the umbilical cords are very close, an inversion of the flow through an arterio-arterial anastomosis can be established. So, instead of bringing the blood pumped through the heart to be oxygenated in the placenta, this vase will now bring the poor blood into oxygen from the other twin, in reverse. The heart of the fetus whose circulation has become reversed ends up becoming hypoxic, culminating, most of the time, with its complete disappearance. The diagnosis is ultrasonographic and can be done during the first trimester morphology, where a fetus with normal morphology is identified and the other one with distorted morphology, characterizing an amorphous mass without identifiable cardiac area. The normal twin may show signs of progressive congestive heart failure due to overload, and this is the criterion for interrupting blood flow to the acardic fetus through coagulation of cord vessels by fetoscopy. Case Report: JRA, 18 years old, was referred to the Fetal Medicine Service of the Presidente Vargas Maternal and Child Hospital at 21 weeks' gestation due to the monochorionic and diamniotic twin pregnancy, where one fetus F2 presented as hydrophilic, polymorphic and without heartbeat. During evaluation, F1 presented increased cardiac area, increased umbilical artery resistance, but with diastole present. F2 did not have an identifiable cardiac area; it was hydropic, individualized cephalic pole presenting ventriculomegaly, unchanged spine. Inverted flow was visualized in one of the umbilical arteries characterizing the TRAP sequence. Three days later, F2 (acardic) had a much increased volume. F1 presented diastole zero in the umbilical artery and venous duct with $\mathrm{IP}=1.04$. Fetal surgery was scheduled sequentially. Fetal death was identified before the procedure could be performed. The fetuses weighed $395 \mathrm{~g}$ and $610 \mathrm{~g}$. Conclusions: The TRAP sequence is commonly described as a monochorionic twin pregnancy where due to chronic hypoxemia the affected fetus presents disruptive malformations in the upper trunk, upper limbs and cephalic pole and is often reduced to an amorphous mass. The reported case alerts to the importance of the earlier diagnosis of this pathology since the acaric fetus will not always present in a classic way.

\section{TWIN PREGNANCY IN A WOMAN WITH UTERINE MALFORMATION: A CASE REPORT}

André Cunha' ', Jorge Telles', Raquel Tomaz', Mariana Lupchinski', Cristine Dietrich', Osvaldo Artigalás ', Daiane Schneider ${ }^{2}$, and Rafael Rosa ${ }^{3}$

${ }^{I}$ Hospital Materno Infantil Presidente Vargas, Porto Alegre, Brazil

${ }^{2}$ Hospital de Clínicas, Porto Alegre, Brazil

${ }^{3}$ Universidade Federal de Ciências da Saúde de Porto Alegre, Porto Alegre, Brazil

Introduction: Twin pregnancies offer pregnant women and physicians a high risk situation due to the increased chance of prema- turity, disruptions and fetal malformations, vascular anastomosis and imperfect twinning, among the most frequent. Uterine defects are the most common malformations in the female reproductive system. The prevalence of uterine malformations varies from 1 to $10 \%$ of women and up to $30 \%$ when only women with abortion are evaluated. The bicornuate uterus accounts for up to $40 \%$ of malformations of Müllerian ducts. It is the most common symptomatic presentation is early and recurrent gestational loss and cervical incompetence. Twin pregnancies in a pregnant woman with uterine malformation further increase the chance of fetal loss and prematurity, although in cases where dizygosis is confirmed, the remaining risks are considerably reduced. We will now report a case of dizygotic twin gestation (including with distinct sexes diagnosed during fetal life) in which each embryo was implanted in one of the horns of a partially duplicated (bicornuate) uterus, thereby diminishing, even if discretely, the chance of prematurity and other complications related to monozygosis. Case Report: A 26-year-old female, HIV-positive, primiparous, was referred to the Fetal Medicine Service of the HMIPV for presenting dichorionic and diamniotic twin pregnancy and anatomical uterine alteration. The first ultrasound evaluation was performed at 19 weeks' gestation, with a uterus with bulging in the fundus region, towards the uterine cavity, with septation in its interior. The maternal kidneys were evaluated, and no changes were identified. After three weeks, normal morphological ultrasonography was performed in both fetuses. The uterine cervix was $3.3 \mathrm{~cm}$ long. It was identified bicornuate womb, being a fetus in each uterine horn. Gestation evolved with the first fetus (F1) with growth in the 50th percentile and the second fetus (F2) developing in the 25th percentile. The patient presented an increase in blood pressure at 37 weeks of evolution and the gestation was interrupted at this moment. The F1, female, weighed 2,560 $\mathrm{g}$ with Apgar score 8/9. The F2, male, weighed 2,615 g at birth with Apgar score 9/9. Conclusions: Although the prognosis of a twin pregnancy in a malformed uterus presents a very reserved prognosis, the implantation of a dichoronic and diamnotic gestation in distinct horns favored the evolution of the gestation.

\section{ANTEPARTUM DEATH OF ONE MONOCHORIONIC CON TWIN WITH CYSTIC ADENOMATOID MALFORMATION}

I. Jimenez Ubieto, B. Pérez Munárriz, L. Martinez Jiménez, M. Huarte Ciganda I. Zabaleta, and I. Martinez Montero

Gynecology and Obstetrics Department, Complejo Hospitalario De Navarra, Pamplona, Spain

Introduction: The death of one twin of a monochorionic pregnancy implies an increased risk for the survivor, which includes death (15\%), preterm delivery $(68 \%)$, pathological neuroimaging $(34 \%)$ and alteration of neurodevelopment $(26 \%)$. The risk is more important than in bichorionic twins because of the intertwin vascular anastomoses. If one of them has a possible death malformation, close prenatal control is compulsory.Case Report: We present the case of a monochorionic twin pregnant woman in her first spontaneous gestation with a normal course of pregnancy. At $20+6$ weeks a cystic adenomatoid malformation (CPAM) was diagnosed in the first twin. The pregnant woman was referred for control at the High Obstetric Risk Consultation. CPAM was similar in size but mild mediastinal shift with mild polyhidramnios was observed. There were no signs of twin-to-twin transfusion syndrome. At 25 weeks, death of the second twin was diagnosed. The pregnant woman referred contractions and cervical length was shortened $(23 \mathrm{~mm})$ so she was admitted in hospital for intravenous tocolityc treatment and fetal lung maturation. Serial ultrasound evaluation of the surviving co-twin was performed. A magnetic resonance imaging at week 29 of gestation did not identify brain injury. No polyhydramnios were 
observed and CPAM decreased. Currently the patient is hospitalized with tocolytic maintenance treatment and progresses favorably. Conclusion: Because of the placental vascular anastomoses between monochorionic twins, the intrauterine death of one twin in a monochorionic pregnancy can cause acute hypotension, anemia, and ischemia in the co-twin due to exsanguination into the low pressure vascular system of the deceased twin, resulting in morbidity or death of the co-twin. In cases of antepartum, fetal death parents should be informed of the risks that this new situation implies. The risk of coagulopathy is very unlikely in these pregnant women, so special surveillance is not considered necessary at this time. On the other hand, the diagnosis of CPAM is suggested by the presence of macrocysts or a solid-appearing mass in the fetal chest on ultrasound examination. The mass appears to resolve before delivery in $50 \%$ of cases. Finally, preterm labor is another risk. In our hospital, in selected cases, maintenance tocolytic is used until $32+6$ weeks.

\section{TWIN PREGNANCY: TIMING AND ROUTE OF DELIVERY - ONE-YEAR EXPERIENCE ON A DIFFERENTIATED PERINATAL CENTER}

Carolina Gomes, João Pinto, Margarida Gadelha, and Ana Paula Silva

Centro Hospitalar Universitário do Algarve - Unidade de Faro, Faro, Portugal

Introduction: The increasing prevalence of twin pregnancy brings concerns about delivery planning and intrapartum management, as several factors influence route and timing of delivery. The aim of this study was to evaluate timing as well as route of delivery for twin pregnancy at a differentiated perinatal center. Methods: This was a retrospective study that included all women referred to Unidade Faro-CHUA with twin pregnancy for surveillance and delivery at our institution during 2016. A total of 51 patients were included. Results: One third of pregnancies occurred in nulliparous women. The majority were dichorionic diamniotic pregnancies $(79.5 \%)$.Mean gestational age at birth was 33 weeks (minimum 28 and maximum 38 weeks and 5 days). Preterm birth occurred in $41 \%$ of dichorionic and $81.8 \%$ of monochorionic pregnancies. Vaginal delivery was achieved in $30.5 \%$. There was one case of cesarean after vaginal birth of the first fetus. The majority of vaginal deliveries occurred when both fetuses were cephalic, with only one case of breech delivery of the second fetus. The remaining deliveries occurred through cesarean (63.6\% elective, mostly for pelvic presentation of the second fetus, followed by non-reassuring fetal heart rate and emergent complications). Spontaneous labor or rupture of membranes occurred in $52.1 \%$ of cases, with $52 \%$ of vaginal deliveries. Cesarean section after spontaneous labor occurred for fetal malposition in $76.9 \%$ and non-reassuring fetal heart rate in $15 \%$. When elective delivery was planned, $8.7 \%$ were under oxytocin-induced labor, resulting in vaginal birth in all cases. The remaining were submitted to elective cesareans, mostly for fetal malposition $(59.1 \%)$. Severe pre-eclampsia with no conditions of induction of labor represented $22.7 \%$ of elective cesareans. Route of delivery was also influenced by chorionicity: $80 \%$ of births in monochorionic pregnancies were through cesarean section, compared to $65 \%$ in dichorionic. No major adverse neonatal outcomes directly related to route of delivery were found. All neonates presented Apgar index $\geq 7$ at 5 minutes. Conclusion: Several concerns arise when planning delivery for multifetal pregnancy, from fetal presentation to emerging complications. Choosing the appropriate timing and delivery route should be carefully discussed, based on best current scientific knowledge but individualized for each specific situation.

\section{DELAYED-INTERVAL DELIVERY IN TWINS: HOW}

\section{SUCCESSFUL?}

Carolina Gomes, Fernanda Vilela, João Pinto, Ana Paula Silva, and Olga Viseu

Centro Hospitalar Universitário do Algarve - Unidade de Faro, Faro, Portugal

Introduction: Multifetal pregnancy is increasing, partially due to medically assisted reproductive techniques. These pregnancies are often associated with more obstetric complications, including preterm delivery and associated fetal and maternal morbidity and mortality. Occasionally, preterm birth of the presenting fetus may not require delivery of the second. As neonatal morbidity is primarily dependent on gestational age at birth, in selected cases, an extended time interval between birth of twins may improve neonatal outcome of the second fetus. Case Report: A 34-yearold nulliparous woman, with a dichorionic diamniotic twin pregnancy achieved after in vitro fertilization, was admitted at our department at 20 weeks and 3 days with preterm premature rupture of membranes of the presenting fetus. Ultrasound revealed fetus A with cardiac activity and anhydramnous, and reassuring wellbeing in fetus B. Antibiotic coverage was started immediately as well as analytic and ultrasound monitoring. At 23 weeks and 5 days, due to contractility and cervical dilation, corticosteroids and tocolysis were initiated. One day later, we confirmed the expulsion of fetus A with no vitality. Delayed-interval delivery was decided, with ligation of the umbilical cord and tight monitoring of infection markers. Five days later, at 24 weeks and 4 days, due to clinical suspicion of chorioamnionitis, cesarian section was performed, with extraction of the second fetus with good vitality, Apgar index 8 at 1 and 5 minutes. Seventy days after birth, the newborn was still in the intensive care unit but with good recovery, despite several complications of severe prematurity. Conclusion: In selected cases of multiple gestation in which the first fetus delivers due to a condition that has not affected the second fetus, prolongation of pregnancy can be done in a closely monitored environment. However, the relative small incidence of cases and the inexistence of large-scale randomized studies make it difficult to advise the couple on the best management and expected prognosis. Therefore, the counseling process should include not only the benefits of delayed-interval delivery, but also the risks for mother or fetus of possible complications.

\section{AN OVERVIEW OF TWIN PREGNANCY COMPLICATIONS: THE EXPERIENCE OF A DIFFERENTIATED PERINATAL \\ CENTER}

Carolina Gomes, Patrícia Soares, Ana Edral, Margarida Gadelha, and Ana Paula

Silva

Centro Hospitalar Universitário do Algarve - Unidade de Faro, Faro, Portugal

Introduction: Multifetal pregnancy is increasing, partially because of growing rates of pregnancy following assisted reproductive techniques. This may be a concern to obstetricians because of the increased number of complications. The aim of this study is to evaluate the incidence of maternal, fetal and peripartum complications in twin pregnancy in a differentiated perinatal center. Methods: This is a retrospective study which included all pregnant women referred to Unidade Faro-CHUA with twin pregnancy for surveillance, during 2016. After exclusion of cases of misdiagnosis and follow-up loss, a total of 51 patients were included. Results: Maternal mean age at pregnancy was 31.9 (SD 5.4). Thirty-five percent of women were nulliparous and $80 \%$ of the gestations were spontaneous. The majority were dichorionic diamniotic pregnancies (79.5\%). Sixtyone percent of women required yard admission $(70 \%$ in monochorionic and $59 \%$ in dichorionic pregnancy), with a mean duration of 12 days, and a mean of 33 weeks of gestational age at admission. The rates of pregnancy complications were $50 \%, 25 \%, 13,7 \%, 6.3 \%$, 
$2.1 \%, 2.1 \%$ and $2.1 \%$ for preterm labor, fetal growth restriction, gestational diabetes, intra-hepatic cholestasis, pre-eclampsia, HELLP syndrome, and intrauterine fetal demise respectively. After birth, $31 \%$ of women had anemia, $14.6 \%$ had increased post-partum bleeding, with $14 \%$ requiring blood transfusion and one case of hysterectomy after uncontrollable post-partum bleeding due to uterine atony. No statistically significant difference was found between di- and monochorionic pregnancy for maternal complications. Mean gestational age at birth was 35.6 weeks ( 34.4 in monochorionic and 36.2 in dichorionic pregnancy), with the earliest delivery at 28 and the latest at 38 weeks. Admission to the Neonatal Intensive Care Unit occurred for both newborns in $29.2 \%$ of cases, this percentage increasing to $60 \%$ in monochorionic pregnancy. Only one newborn requiring admission occurred in $23 \%$ of cases. Mean duration was 10 days, with maximum 71 days. Most frequent causes for admission included, for both groups, low birth weight, respiratory distress syndrome, early and late sepsis and jaundice. No neonatal deaths occurred. Conclusion: Multifetal pregnancy represents a challenge for obstetric care. In such pregnancy, maternal and fetal morbidity and mortality are increased, requiring surveillance in differentiated outpatient consultation as well as prolonged staying in inpatient care.

\section{PATHOLOGICAL NEUROIMAGING AS A CONSEQUENCE OF THE FETAL DEATH OF A TWIN}

Lara Martínez Jiménez, Izarbe Jiménez Ubieto, María Huarte Ciganda, Beatriz Pérez Munárriz, Ana Pérez Rodriguez, and Natalia Abian Franco

Obstetrics Department, Complejo Hospitalario de Navarra, Pamplona, Spain

Introduction: Twin pregnancy is associated with higher rates of almost every potential complication of pregnancy. Monochorionic twins have a higher rate of congenital anomalies than dichorionic twins and singletons. The anomalies have a high rate of concordance, but can be discordant. Single fetal demise of one twin of a monochorionic pair can cause morbidity or mortality in the cotwin due to their shared placental circulation. The risks to the surviving twin of death or neurological abnormality are of the order of $15 \%$ and $26 \%$ respectively. Material and Methods: We present the case of a monochorionic twin pregnancy discordant for fetal anencephaly. The malformed fetus died spontaneously intrauterine at 15 weeks of pregnancy. Results: We present the case of a monochorionic twin pregnancy with no history of interest. First-trimester ultrasound shows the first fetus with a nuchal translucency in the 95th percentile with no other markers of chromosomal abnormalities and the second fetus with severe morphologic abnormalities (anencephaly). An umbilical cord oclusion for selective feticide was offered to the patient. However, the affected fetus died spontaneously at 15 weeks of pregnancy. Amniocentesis of the healthy fetus is performed to rule out chromosomal abnormalities resulting from normal female fetal karyotype.

Subsequent control ultrasounds are normal, with adequate fetal growth and no major fetal morphological abnormalities of the surviving fetus. A magnetic resonance imaging is performed at week $21+6$ showed moderately dilated occipital horns and signs of occipital parenchymal atrophy for probable chronic ischemic injury. An ultrasound confirmed the lesions of the occipital region, with a suspected diagnosis of esquisencephaly. The patient, informed about the fetal risks associated with the diagnosis, decided to terminate the pregnancy at 22 weeks. Conclusions: In cases of antepartum fetal death, parents should be informed of the risks that this new situation implies. Death of one twin of a monochorionic pair may have direct harmful effects on the survivor because of intertwin vascular anastomoses. Fetal magnetic resonance imaging of the brain may be performed 4 weeks after co-twin demise to detect neurological morbidity if this information would be of value in planning management.

\section{HORMONE REPLACEMENT THERAPY - RELATED DNA METHYLATION, GENE EXPRESSION AND MIR PROFILES ASSOCIATE WITH WITHIN-PAIR DIFFERENCES IN CLINICAL MEASURES}

Miina Ollikainen' ', Reeta Kangas², Aileen Bahl', Timo Törmäkangas ${ }^{2}$, Jaakko Kaprio', Taina Rantanen ${ }^{2}$, Vuokko Kovanen ${ }^{2}$, and Eija Laakkonen ${ }^{2}$

${ }^{1}$ Institute for Molecular Medicine Finland, and Department of Public Health, University of Helsinki, Helsinki, Finland

${ }^{2}$ Gerontology Research Center, Faculty of Sport and Health Sciences, University of Jyväskylä, Jyväskylä, Finland

Introduction: The menopausal loss of estrogen causes changes in the female body with wide-ranging effects on health. Hormone replacement therapy (HRT) relieves menopausal symptoms and is associated with tissue-specific gene expression profiles. As gene expression is controlled by epigenetic factors, part of the HRT-associated gene expression may be due to changes in DNA methylation. MicroRNAs (miRs) are another class of epigenetic regulators that affect gene expression and associate with HRT. Exosomal (exo) miRs participate in intercellular messaging, and can be used to reflect the physiological status of an individual. Materials and Methods: The study participants $(n=20 \mathrm{MZ}$ HRT discordant female twin pairs) originate from two Finnish twin studies, SAWES and FITSA, for which the study subjects were recruited from the Older Finnish Twin Cohort. We investigated white blood cell (WBC) genomewide DNA methylation (Illumina 450k BeadChip) and gene expression (Illumina Human-WG6 BeadChip) and serum exomiR profiles (small RNA sequencing by Illumina MiSeq V3), and their associations with body composition and blood biochemistry. Results: We identified 4,044 genes with nominally significant differentially methylated regions (DMRs). Five of these genes (ACBA1, CCL5, $F A S L G, P P P 2 R 2 B$, and $U H R F 1$ ) were also differentially expressed. All have been previously associated with HRT or estrogenic regulation, but not with HRT-associated DNA methylation. All five genes were associated with bone mineral content, and ABCA1, FASLG, and $U H R F 1$ were also associated with body adiposity. We detected 241 exomiRs by next generation sequencing, 14 showing HRTassociated differences within the twin pairs. Differentially expressed miRs were predicted to affect cell proliferation showing inactivation with HRT usage. The hierarchical cluster analysis revealed significant association with multitude of clinical (including 17 $\beta$-estradiol, HDL, LDL) and body adiposity measures revealing positive and negative exomiR clusters. Conclusions: HRT associates with DNA methylation alterations in WBCs and exomiR content in serum. We show that five differentially expressed genes with DMRs and six exomiRs associate with body fat and clinical measures. Thus at least part of the HRT effects may be regulated by DNA methylation associated alterations in gene expression in circulating WBCs. Furthermore, the individual benefits of HRT use could be evaluated based on the better or worse serum exomiR signature.

\section{EPIGENETIC SIGNATURES OF PRENATAL GROWTH CONTROL}

Marij Gielen' ${ }^{1}$ Aafke Van Montfort ${ }^{2}$, John Dumoulin² ${ }^{2}$ Catherine Derom ${ }^{3}$, Kasper Derks $^{4}$, and Han Brunner ${ }^{4,5}$

${ }^{I}$ Maastricht University Medical Center, Complex Genetics, Maastricht, the Netherlands ${ }^{2}$ Maastricht University Medical Center, Obstetrics and Gynaecology, Maastricht, the Netherlands

${ }^{3}$ Ghent University Hospitals, Department of Obstetrics and Gynaecology, Ghent, Belgium

${ }^{4}$ Maastricht University Medical Center, Clinical Genetics, the Netherlands

${ }^{5}$ Radboud University Medical Center, Nijmegen, the Netherlands

Introduction: We recently made four key observations to indicate that epigenetic changes play a role in embryonic and fetal growth: (1) Two different media used for IVF embryo culture differ significantly in their outcome with regards to birth weight; (2) Differences in mRNA gene expression profiles exists between human 
preimplantation embryos cultured in these two different IVF culture media; (3) Data from the East Flanders Prospective Twin Survey (EFPTS) show that MZ twins discordant for birth weight remain discordant for height at adult age and that (4) Placentation and umbilical cord insertion predict birth weight. We start a new project in December 2017 which will unravel how DNA methylation patterns influence growth from conception to birth and beyond. The key objectives are: (1) Study the effect of different ART culture media on DNA methylation patterns in human blastocyst embryos. (2) Establish placental DNA methylation patterns in a large MZ twin population discordant for birth weight. (3) Investigate the influence of environmental factors in the periconception period (natural conception vs ART; different ART culture media) and later gestation (placentation) on placental DNA methylation. (4) Examine the long term relationship between placental DNA methylation patterns and growth up to adulthood, using follow-up samples of twins of the EFPTS. Methods: Genome wide DNA methylation will be assessed in 20 spare embryos donated by patients after IVF treatment and subsequently grown to blastocyst stage. Placental DNA methylation will be assessed in $150 \mathrm{MZ}$ natural and ART conceived twin pairs severely discordant for birth weight selected from the EFPTS stratified by chorionicity (MC vs. DC). For a subset of $20 \mathrm{MZ}$ twins, we shall compare methylation patterns in multiple tissues (placenta, cord blood and buccal swabs) from the same individual. Buccal swabs at adult age allow comparing methylation profiles from birth to adulthood and relating this to adult height and weight. We shall mainly use the Illumina Infinium Human Methylation array, complemented with occasional whole genome bisulphite sequencing. For specific loci and genes, we shall perform mRNA sequencing to relate DNA methylation to gene expression. Relevance: Understanding how the prenatal period shapes the human epigenome and which genomic regions are sensitive to environmental influences will allow us to distinguish a healthy start of a new life from an unfavorable start.

\section{FETAL TACHYCARDIA IN TWIN PREGNANCY}

Ángela Uceda' ', Lucía Deiros', Elisa Castaño', María De La Calle Fernández², Roberto Rodríguez ${ }^{2}$, Federico Gutiérrez-Larraya', and José Luis Bartha ${ }^{2}$ ${ }^{1}$ Pediatric Cardiology Department, University Hospital La Paz, Madrid, Spain ${ }^{2}$ Gynecology and Obstetrics Department, University La Paz, Madrid, Spain

Introduction: Fetal arrhythmias are a common reason for consultation in prenatal cardiology. Among the potentially serious ones, the majority are supraventricular tachycardias (SVT), which may be associated with fetal heart failure and hydrops. Close monitoring is required and the need for medication is frequent. These treatments pose an ethical dilemma, since it involves subjecting a healthy patient, the mother, and the healthy fetus, to possible adverse effects. Case 1: Controlled twin pregnancy. In the center of origin it was detected at week 26 in fetoA TSV 270 lat / min, driving 1/1. FetoB in sinus rhythm, without alterations. Close follow-up with progressive development of pericardial effusion in fetus, so that at week 28 , maternal treatment with digoxin was started. At our center (sem30), continuous digoxin without rhythm control, maximum maternal levels of 1.6. At that time the Doppler record showed interval VA $>\mathrm{AV}$ and flecainide was added to the treatment with daily monitoring of mother and both fetuses. FetoA: intermittent sinus rhythm after 72 hours of bitherapy and stable at week 32, with disappearance of the effusion. FetoB: slight increase in AV interval with medication. Mother: QTc interval prolongation that normalizes after reducing flecainide. Cesarean in week $36+5$. Case 2 : Controlled twin pregnancy. In center of origin it was detected in week 27 in fetoA TSV 277 lat / min, driving 1/1. FetoB in sinus rhythm, without alterations. In the follow-up progressive development of pleural effusion and ascites in FetoA and is derived. At our center (without 29) tachycardia maintained with fetal hydrops and severe ventricular dysfunction, maternal treatment with digoxin is initiated (maternal maximum levels of 1.8). At 72 hours, rhythm control was not achieved and flecainide was associated. Daily monitoring of mother and both fetuses. FetoA: cardioversion at 5 days with improvement of ventricular function and disappearance of pleural effusion. FetoB: slight increase in AV interval. Mother: QRS prolongation. Cesarean in week 31. Conclusion: Treatment of fetal tachycardia is not standardized in twin gestation. There are very few cases in the literature, and the effects of antiarrhythmics on healthy fetuses are not well defined. Each case should be individualized, assessing risk-benefit, with close monitoring to detect early side effects in the healthy fetus while controlling the tachycardia in the arrhythmic fetus.

\author{
THE CHALLENGES FACED BY TWINS, MULTIPLES AND \\ THEIR FAMILIES: EXPERIENCES IN THE UK AND

\section{AUSTRALIA} \\ Keith Reed', Lucas Calais Ferreira ${ }^{2,3}$, Monica Rankin ${ }^{4}$, Jeffrey M. Craig, ${ }^{5,6}$, and \\ Deborah Osborne ${ }^{2}$ \\ ${ }^{I}$ Twins and Multiple Births Association, London, UK \\ ${ }^{2}$ Centre for Epidemiology and Biostatistics, Melbourne School of Population and \\ Global Health, The University of Melbourne, Melbourne, Australia \\ ${ }^{3}$ CAPES Foundation, Ministry of Education, Brasilia, Brazil \\ ${ }^{4}$ International Council of Multiple Birth Organisations, Melbourne, Australia \\ ${ }^{5}$ Murdoch Childrens Research Institute, Royal Children's Hospital, Melbourne, \\ Australia \\ ${ }^{6}$ Department of Paediatrics, The University of Melbourne, Melbourne, Australia
}

Introduction: Twins, multiples and their families face significant challenges. These include a higher rate of preterm birth, low birth weight births, and pregnancy-related complications compared to families of singletons. In addition, parents of multiples have higher rates of psychiatric conditions such as post-partum depression and stress that might put a long-term pressure on the whole family. Ongoing difficulties with twins' health and development can result in a larger financial burden for parents of twins and multiples compared to those of singletons. Challenges include lower than average income and material deprivation, mainly due to mothers returning to work much later and parents having to work longer hours than with singletons. A lack of understanding of major issues of zygosity and its emotional impact on twins and their families have also been reported. To address these challenges, it is important that an integrated approach between a variety of stakeholders is taken. More importantly, twins, multiples and their families need to be part of the process of shaping future research on these topics to provide a valid framework for public discussion and policy-making. The Forum: The Twins and Multiples Community Forum (TMCF) is a joint initiative by Twins Research Australia, the Twins and Multiple Births Association (UK), the Australian Multiple Birth Association, and the International Council of Multiple Birth Organisations. It draws from different sets of expertise of each of these organisations aimed at providing pathways for engagement between the twin and multiples community, researchers and health professionals. The organisation of a half-day forum on 26 October 2017 in Melbourne, Australia, and the development of a White Paper to shape public conversations and policy-making addressing the social and health inequalities of this group are the first steps to achieve this goal. The initiative aims to establish strong links between the three aforementioned stakeholder groups and to provide a space for collective learning and sharing of knowledge unique to the Australian and British contexts but relevant to the broader international community. It is hoped that the outcomes arising from the Forum will also be relevant to addressing the disadvantages of families of twins and multiples in other countries. 


\section{BUILDING NETWORKS, CAPACITY AND THE NEW}

\section{WORKFORCE IN GLOBAL TWIN RESEARCH}

\author{
Lucas Calais-Ferreira ${ }^{1,2}$, Ada Castle $^{1}$, Aline Jelenkovic ${ }^{3}$, Ally Avery $^{4}$, Amie \\ Hwang ${ }^{5}$, Bianka Forgo ${ }^{6}$, Hamdi Mbarek $^{7}$, Jenny Van Dongen ${ }^{7}$, Karin Magnusson ${ }^{8,9}$, \\ Kaushalya Jayaweera ${ }^{10}$, Lucia Colodro-Conde ${ }^{11,12}$, and Paraskevi Christofid \\ ${ }^{I}$ Centre for Epidemiology and Biostatistics, Melbourne School of Population and \\ Global Health, The University of Melbourne, Melbourne, Australia \\ ${ }^{2}$ CAPES Foundation, Ministry of Education, Brasilia, Brazil \\ ${ }^{3}$ University of Helsinki, Helsinki, Finland \\ ${ }^{4}$ Elson S. Floyd College of Medicine, Washington State University, Spokane, USA \\ ${ }^{5}$ Department of Preventive Medicine, Keck School of Medicine, University of Southern \\ California, Los Angeles, USA \\ ${ }^{6}$ Semmelweis University, Budapest, Hungary \\ ${ }^{7}$ Department of Biological Psychology, Vrije Universiteit Amsterdam, Amsterdam, the \\ Netherlands \\ ${ }^{8}$ National Advisory Unit on Rehabilitation in Rheumatology, Dept. of Rheumatology, \\ Diakonhjemmet Hospital, Oslo, Norway \\ ${ }^{9}$ Clinical Epidemiology Unit, Orthopaedics, Dept. of Clinical Sciences, Lund \\ University, Lund, Sweden \\ ${ }^{10}$ Institute for Research and Development, Colombo, Sri Lanka \\ ${ }^{11}$ QIMR Berghofer Medical Research Institute, Brisbane, Australia \\ ${ }^{12}$ University of Murcia, Murcia, Spain \\ ${ }^{13}$ Department of Twin Research and Genetic Epidemiology, King's College London, \\ London, UK \\ ${ }^{14}$ School of Health Sciences, Central Michigan University, Mount Pleasant, USA
}

Introduction: The International Network of Twin Registries (INTR) was established in 2011 as a working group of the International Society for Twin Studies (ISTS), aimed at facilitating international collaboration and promoting twin research globally. The INTR EarlyCareer Group was recently created to support those objectives and audit twin registries and cohorts to build a searchable catalogue of existing twin data and biospecimens that are available for future ethically approved studies. Materials and Methods: For the ongoing audit's first stage, we have designed a questionnaire to collect information on the twin registries' overall profile and sample characteristics, working with twin registries and senior researchers. We are currently expanding data collection to other twin registries and will also present strategies to involve more twin registries and disseminate results more widely. Sample characteristics describe numbers of complete twin pairs and higher order multiples including breakdowns of zygosity, sex, age, along with information on numbers and types of available biospecimens. The second stage (in planning) will collect information at the variable level and will include more in-depth characteristics of available datasets, such as information on a variety of anthropometric and lifestyle variables, disease-specific risk factors and outcomes, and epigenetic, GWAS and microbiome analyses. Results: The ongoing pilot of the audit's first stage includes information on 13 twin registries and cohorts in Australia, Brazil, Finland, Hungary, Iran, Malaysia, the Netherlands, Spain, Sri Lanka, South Korea, United Kingdom and the United States. More than 100,000 complete twin pairs have been audited so far, and the zygosity, sex and age range of those pairs were recorded. Other information on the twin registries and cohorts' such as recruitment strategies and availability and type of biospecimens were also collected. Conclusions: The project will bring an innovative approach to data and knowledge sharing to conduct new multi-center twin studies with large sample sizes. It will open doors for researchers and professionals who are not yet involved in twin research. We also aim to build a catalogue of researchers who have the expertise and/or interest in working with twin data so new collaborations can be formed. We will discuss our plans and their implications for the further development of the twin research workforce and the role of the INTR Early-Career Group in these endeavours.

\section{ADOLESCENCE BODY MASS INDEX AND RISK OF EARLY} MENARCHE: A MENDELIAN RANDOMIZATION STUDY

\author{
Seung-Ah Choe', Hakyung $\mathrm{Kim}^{2}$, and Joohon Sung ${ }^{3}$ \\ ${ }^{I}$ Department of Obstetrics and Gynecology, School of Medicine, CHA University, \\ Gyunggi-do, South Korea; Department of Epidemiology, Brown University School of \\ Public Health, Providence, RI, USA \\ ${ }^{2}$ Department of Epidemiology, School of Public Health, Seoul National University, \\ Seoul, South Korea \\ ${ }^{3}$ Department of Epidemiology, School of Public Health, Seoul National University \\ Seoul, South Korea; Institute of Health and Environment, Seoul National University, \\ Seoul, South Korea.
}

Introduction: Decreasing trend of age at menarche has been reported globally. Early menarche is known to be a major risk factor for early sexual debut, adolescent pregnancy, premature menopause and breast cancer. Although the exact mechanism which triggers the initiation of puberty is not clear, adolescence obesity is suggested to play a role in this reproductive transition. The purpose of this study was to explore the causal relationship between adolescence body mass index (BMI) and early menarche. Materials and Methods: Data of the Korean Genome and Epidemiology Study (KoGES) conducted from 2004 to 2013 were used. Early menarche was statistically defined considering the general trend of lowering age at menarche. Among the women who reported their age at menarche and body weight at the age of 18-20 years, those whose age at menarche was below the 3th percentile for the population of the same birth cohort were classified to have early menarche. We replicated a genome-wide association study (GWAS) of early menarche and adolescence BMI in the study population. Results: A total of 118,623 Korean women who aged $\geq 20$ years were included in the analysis. In multivariable logistic regression analysis, the risk of early menarche increased with higher adolescent BMI (Adjusted odds ratio $[O R]=1.27,95 \%$ confidence interval $[\mathrm{CI}],[1.19,1.37])$. Using a genetic risk score to indicate early menarche, we found that higher risk of early menarche with increasing of adolescence BMI (adjusted $O R=1.003,95 \%$ CI $[1.000,1.067]$ ). Conclusion:This finding suggests a possible causal relationship between higher adolescence BMI and increased risk of early menarche.

\section{GENETICS AND ENVIRONMENTS ON AGE AT MENARCHE}

Hakyung Kim, Seung-Ah Choe, and Joohon Sung

${ }^{I}$ Complex Disease \& Genome Epidemiology Branch, Department of Public Health, Graduate School of Public Health, Seoul National University, Seoul, Korea ${ }^{2}$ School of Medicine, CHA University / Graduate School of Public Health, Brown University

Introduction: The menarche age is known as a risk factor of increasing health problems. In developed countries, descending trend of the average age of menarche has observed. Although determinants of menarche age is not established, it has been suggested that genetic and childhood obesity are associated with menarche timing. In this study, we aimed to estimate genetic contributions and evaluate genetic correlations between age at menarche and obesity at adolescence. Method: From the Healthy Study, Korea (HTS) $(n=3,499)$, a twin-family cohort study, we selected 1,567 individuals who had been answered to the questions of recalled menarche age. For inter-generational comparisons, we formulated a normalized premature menarche score (NPM score. Among the same age group (10-year scale) using a large population data in Korea (the Korean Genome and Epidemiology Study, $n=122,883$ ) as reference, we calculated the percentile value in the quantile menarche age. This percentile menarche age was transformed into NPM score by equation as follows: NPM score $=$ square root (percentile of individual). Body mass index (BMI) at age 18-20 was used for the proxy of childhood obesity. The heritability is estimated for NPM score after adjusting age, level of education and young adulthood BMI. Variance component approaches in the SOLAR were used for analysis. Results: NPS score was different by age $(p=.05)$, 
educational categories $(p<.0001)$ and adolescence BMI $(\mathrm{p}=$ 0.002). Each age group showed similar distribution of NPM score even if mean age at menarche were differ. For scale menarche age, ACE was best fitted model $(\mathrm{A}=0.33, \mathrm{C}=0.28, p<.00001)$. In bivariate analysis, genetic and environmental effect correlation were estimated between menarche age scale and adolescence BMI. Menarche age scale and adolescence BMI has small genetic correlation $(\mathrm{RhoG}=0.24)$ which was significantly different from complete pleiotropy effect $(p=.03)$ and no environmental correlation (RhoE $=0.1, p=.29$ ). Conclusion:Our finding suggest that NPM score is useful indicator of risk of early menarche age in data with different age group to avoid confounding effect of menarche age trend of age cohort. The results indicate that conventional genetic effects might play moderate roles on menarche age. There was no correlation in both genetic and environment factor between two variables. Since there is no correlation factor, adolescence BMI may be considered as a risk factor of menarche age in further analysis.

\section{A QUALITY IMPROVEMENT PROGRAM IN ENGLAND AIMED AT IMPROVING ANTENATAL CARE FOR MULTIPLE PREGNANCIES}

Keith Reed, Amy McCarthy, Helen Turier, Jane Gorringe, Deborah Dillion, and Melanie Burley

Twins and Multiple Births Association (Tamba), London, UK

Multiple birth babies make up just $1.5 \%$ of births in the United Kingdom but account for $7 \%$ of still births and $10 \%$ of neonatal deaths, with more than six times the risk of cerebral palsy than in singleton pregnancies. The risk of preterm birth is also considerably higher, occurring in at least $50 \%$ of twin pregnancies. Since 2005 the number of patient safety incidents involving multiple pregnancies has risen by $419 \%$. From $2005-2010$ alone, multiple birth safety incidents resulted in payouts totaling $£ 93$ million and accounted for $10 \%$ of all maternity cases handled by the National Health Service (NHS) Litigation Authority. There are changes happening in the NHS that focus on making care for pregnant women even better. As part of this work, Tamba received financial support from the Department of Health to deliver a quality improvement program that seeks to encourage greater use of national clinical guidance. The guideline 'Multiple pregnancy: antenatal care for twin and triplet pregnancies [CG129]' was promoted and produced by the National Institute for Health and Care Excellence and published in 2011. According to a report by Tamba and the National Childbirth Trust, only $69 \%$ of maternity units in the United Kingdom were using it, based on parental experience of care during 2014/15. The project started in April 2016. A project team has been recruited and includes a full-time project manager, two part time coordinators, a part-time audit and evaluation officer, as well as a team of specialist consultant midwives. The team are supported by a steering group of experts. Members of the group include clinicians from St George's (London), Leeds and Liverpool, and representatives from the relevant Royal Colleges, Government bodies, Care Quality Commission, University of Leicester, and other charities. The group meets quarterly to provide the project team with expertise in overcoming challenges that arise during the program. 28 maternity units have agreed to take part, varying in size, location and at different stages of implementing the guidance. The units were shortlisted using information from both Tamba's Maternity Services Report 2015 and also from nationally published rates of stillbirth and neonatal deaths. The units work with Tamba to help develop the service they offer families through undertaking audits, implementing bespoke improvement packages and receive ongoing support. Early findings from the programme are now available with a final report due in 2019 .

\section{COMPLICATIONS DURING COMPLETE BREECH EXTRACTION IN AN ACTIVE MANAGEMENT PROTOCOL INTRODUCTION}

Jose Adánez García, Oscar Vaquerizo Ruiz, Marina Navarro López, Ana Escudero Gomis, Carmen Gutiérrez-Cecchini Pérez, and Javier Ferrer Barriendos Obstetrics and Gynecology Department, The Central University Hospital of Asturias, Oviedo, Spain

Introduction: Protocolized complete Breech Extraction of the second twin was implemented in 2010 in Asturias Central University Hospital. The purpose of this study is to analize the obstetrics complications of the procedure and to describe the neonatal outcomes. Material and Methods: We conducted a retrospective study that included 47 complete breech extraction of the second twin assisted at our hospital between 2010-11 and 2014-16. Population data were: mean maternal age 34,7 (27-44), gestational age 35 weeks (30-40), and $42.6 \%$ of the patients were nulliparous. The onset of the trial of labor was spontaneous for $44.4 \%$, and $56.3 \%$ were induced. Fetal presentation before labor was mainly first twin in cephalic presentation and second twin in non-cephalic $(80.9 \%)$, cephalic/cephalic $(17 \%)$ and both podálica $(2.1 \%)$. Training with simulation using mannequins was implemented for the assistance of the second twin delivery and the management of its complications: failed fetal extraction and cesarean of the second twin, nuchal arm, interglute umbilical cord loop and entrapment of the aftercoming head. $R e$ sults: Patients selected for a trial of labor achieved a vaginal delivery in $84.2 \%$ of the cases. Apgar tests were $>7$ in all second twins. Mean umbilical cord arteria pH was 7.28 (7.15-7.38) and mean fetal weight was $2,357 \mathrm{~g}(1,430-3,695)$. Obstetric complications that required additional manouvers during the extraction were nuchal arm in 4 cases $(8.5 \%)$ and interglute umbilical cord loop in $2(4.2 \%)$. We observed no cases of entrapment of the aftercoming head, failed fetal extraction or serious fetal morbidity. Conclusions: Active management of the second twin delivery is safe. Periodic training on simulation is recomended.

\section{'CHALLENGES' AND ‘THINGS THAT HELP' IN PARENTING} MULTIPLE BIRTH CHILDREN

Setsuko Ochiai ', Naoko Naito ${ }^{2}$, Emi Otaka $^{3}$, Yuko Ochi $^{4}$, Anna Matsukawa $^{5}$,

Hiroko Ogishi ${ }^{6}$, Chika Hashimoto ${ }^{7}$, and Shigeo Tatsuki ${ }^{1}$

${ }^{\prime}$ Doshisya University, Kyoto,Japan

${ }^{2}$ Ningenkankyo University, Aichi, Japan

${ }^{3}$ The Japanese Red Cross Akita College Nursing, Akita, Japan

${ }^{4}$ Nagoyagakuin University, Aichi, Japan

${ }_{5}^{5}$ Japan Multiple Births Association, Hyogo, Japan

${ }^{6}$ Disaster Reduction and Human Renovation institution, Hyogo, Japan

${ }^{7}$ Yodomachi Station,Osaka, Japan

Introduction: Parenting multiple birth children (multiples) is characterized by the need to care for more than one child of the same age 24 hours per day, every day. Consequently, from pregnancy through the childcare period, the support of family, hospitals, local health and welfare services and communities is important. By clarifying what those raising multiples see as the 'challenges' and 'things that help', this study examines support for multiples childcare from the perspective of social capital. Material and Methods: Participants were 33 members of five mother's circles across Japan. There were 6-8 participants at each site. The period of the study was July to October 2015. The method used was workshops using the KJ method as a form of CBPR. The two items in the study were 'challenges' and 'things that help'. Data analysis was conducted by putting affinity diagrams created by participants into data form, and by the researchers then reconstructing and analyzing the affinity diagrams from all five locations. Results: In total, 367 opinion cards for "challenges' were obtained. The following four categories emerged from the affinity diagrams reconstructed by the researchers: (1) mothers being so tired looking after more than one child of the same age with the accompanying financial burden and not having the 
physical capacity to do everything that they did not have the time and space to appreciate how lovely their children were (312), (2) being a minority in the childcare space, leading to a lack of information about pregnancy and childcare and lack of childcare goods for multiples, difficulties in making friends with other mothers, and lack of understanding and empathy from others regarding raising multiples (19), (3) concerns about premature birth, NICU treatment and developmental delays (13), and (4) lack of cooperation and consideration from family, dealing with siblings (21). In total, 220 'things that help' opinion cards were obtained. 'Things that help' were classified as (1) cooperation through the power of being twins, (2) cooperation of family and neighbors, (3) mutual help within the circle, (4) public assistance, and (5) self-help. 'Cooperation through the power of being twins' accounted for $41.4 \%$ of the opinions. Conclusions: This study once again suggests that human networks together with norms of trust and norms of reciprocity are the foundation for considering the 'challenges' and 'things that help' in parenting multiple birth children.

\section{FROM GENES TO BEHAVIOR THROUGH SEX HORMONES AND SOCIALIZATION: THE EXAMPLE OF GENDER DEVELOPMENT}

Sheri A. Berenbaum and Adriene M. Beltz

Departments of Psychology \& Pediatrics, The Pennsylvania State University,

Department of Psychology, University of Michigan, USA

Introduction: Irving Gottesman made clear that behavior was the result of a complex interplay between genes and environment across development, and he was keen to understand the pathways by which genetic predispositions become enacted in behavior through the brain and an individual's transactions with the social world. Although he is most known for his work on psychopathology, Irv was interested in psychological development broadly. His imprint is seen in our work: Gender development represents a prime opportunity to how biology (particularly sex hormones) and socialization work together to influence behavior. Method and Results: We will review previous studies and recent data showing how psychological development is influenced by prenatal androgen levels. Data come from multiple sources: individuals with disorders of sex development (in which prenatal androgen levels are sex-atypical, and unconfounded with rearing sex); typical individuals in which variations in amniotic hormones or genital anatomy are linked to postnatal behavior; and comparisons of opposite-sex and same-sex dizygotic twins. (Although digit ratio has been used as a marker of prenatal androgens in behavioral studies, it is not a valid index of individual differences in prenatal androgen exposure.) Evidence clearly shows that prenatal androgens affect human sex-related psychological characteristics in varying ways: They have large effects on activity interests and participation, moderate effects on spatial abilities and some aspects of personality (such as aggression), and small or no effects on gender identity, gender cognitions, and gendered peer interaction. These differential effects provide an opportunity to move beyond identifying sources of variation in psychological outcomes to understanding developmental processes, including links among gendered characteristics, psychological and neural mechanisms underlying development, and joint effects of biological predispositions and social experiences. Conclusions: Gender development represents an excellent example of the joint effects of nature and nurture. Hormones represent important pathways by which genes affect behavior, through effects on the brain and an individual's transactions with the social world. Studies of same- and opposite-sex twins provide a novel opportunity to study this issue. This work shows the broad reach of Irv Gottesman's ideas and mentoring.

\section{PREVENTION AND MANAGEMENT OF PPH IN MULTIPLE} PREGNANCY

\author{
Anton Mikhailov ${ }^{1,2,3}$, Vera Shman', Aleksandr Kuznetsov ${ }^{1,2}$, Artem \\ Romanovsky,3, and Anna Shlykova ${ }^{1,3}$ \\ ${ }^{I}$ Maternity clinic \#17, Saint Petersburg, Russia \\ ${ }^{2}$ Pavlov First Saint Petersburg State Medical University, Saint Petersburg, Russia \\ ${ }^{3}$ North-Western State Medical University named after I.I. Mechnikov, Saint Petersburg, \\ Russia
}

Introduction: Postpartum hemorrhage (PPH) is still a major reason for maternal morbidity and mortality in both developed and developing countries. The incidence of PPH, blood transfusion and hysterectomy rates are significantly higher in multiple pregnancy than in singleton. Despite on the way of delivery immediate recognition through the correct estimation of rapidity and volume of blood loss is crucial for further management. Materials and Methods: The rate, total volume and outcomes of PPH were evaluated in 275 multiple deliveries (3.8\% out of all pregnancies $-n=7232$ ) occurring within 18 months (2014-2015). In that period blood loss was estimated using simple visual estimation. Rates were then compared with those that occurred after introducing the gravimetrical method of blood loss estimation -301 multiple deliveries $(4.8 \%-n=$ 7232) within an 18-month period (2016-2017). Results: The rate of PPH dramatically increased from 9.8 to $15.2 \%$ after introducing the gravimetrical method for estimation of total blood loos. Blood loss of less than $500 \mathrm{ml}$ (or 1,000 $\mathrm{ml}$ in CS) has changed from $16 \%$ (20/125) to $13.6 \%$ (24/176). However, in case of $1000 \mathrm{ml}$ equal blood loss range in both delivery ways, $\mathrm{PPH}$ rate was similar -12.8 and $13.6 \%$ with average blood loss volume of $1,574 \mathrm{ml}$ and 1,630 $\mathrm{ml}$ respectively. The rate of massive hemorrhage $(>1,500 \mathrm{ml})$ was almost the same with $4.8 \%$ and $5.1 \%$ respectively. There were 37 cases of oxytocin administration. Induction of labor was performed in 19 cases, oxytocin augmentation administered in 12 cases and 6 cases of CS. The rate of bleeding in oxytocin group was $26.3 \% 25 \%$ and $33 \%$ respectively. In 10 cases of massive PPH external elastic uterine compression waas performed as a first step of PPH surgical management. Uterine elastic compression alone was successful in three cases; in four cases it combined with uterine artery ligation and in three cases compression sutures were applied. In none of the cases were signs of hemorrhagic shock and DIC or necessity of hysterectomy registered. Conclusions: The risk of PPH is significantly higher in multiple pregnancy than in singleton regardless of delivery method. Oxytocin administration is an independent risk factor for PPH. Despite the increase in PPH rate accurate estimation of blood loss allowed to preserve reproductive function. Managing the massive bleeding requires a skilled multidisciplinary approach.

\section{THE USE OF CERVICAL PESSARY IN A SPANISH POPULATION OF TRIPLET PREGNANCIES}

Maria De La Calle', Silvia Arévalo², Nuria Martinez', Carlota Rodo ${ }^{2}$, Eugenia Antolin', Elena Carreras' ${ }^{2}$, and Jose Luis Bartha'

${ }^{1}$ Maternal-Fetal Unit, Obstetrics Department, Universitary La Paz Hospital, Madrid, Spain

${ }^{2}$ Maternal-Fetal Unit; Obstetrics Department, Universitary Vall d'Hebron, Barcelona, Spain

Introduction: Preterm birth is the main cause of perinatal morbidity and mortality, especially in multiple pregnancies. In past years, cervical pessary has been tested as an effective intervention to prevent prematurity in patients with a shortened cervix during pregnancy. Our objective is to describe the outcomes in a Spanish population of patients carrying a cervical pessary during a triplet pregnancy. Methods: A retrospective observational study of triplet pregnancies was followed at the Obstetrics Departments of Hospital Universitari Vall d'Hebron (Barcelona) and Hospital Universitario La Paz (Madrid) from 2009 to 2014. Transvaginal ultrasound scans were periodically performed for evaluation of the cervical length (CL). Patients with 
$\mathrm{CL}<25 \mathrm{~mm}$ and no uterine contractions were offered a cervical pessary. We describe the characteristics of this population and the outcomes for the groups with and without pessary. Results: Between 2009 and 2014, there were 70 triplet pregnancies surveyed in either of the two centers involved in the study. Of these, 24 (34.3\%) patients met the criteria and gave their consent for the insertion of a cervical pessary. The mean gestational age for the insertion was 24 weeks (range 20-30). There were no statistical differences between groups for chorionicity, mode of conception, need for admission at the hospital or indication for admission at the hospital. Mean CL was significantly shorter in the pessary group $(22.2 \mathrm{~mm}, 95 \% \mathrm{CI}$ $[20.5,23.9]$ vs $33.4 \mathrm{~mm}, 95 \%$ CI [32.1, 34.6]). Mean gestational age at birth was 32.2 weeks (95\% CI $[31.4,33.1])$, with no statistical differences between groups. Regarding the indications to end the pregnancy (all of them by cesarean section), there were $25 \%$ of patients with preeclampsia or Doppler alterations in the non-carriers group (vs. $0 \%$ in the carriers group), differences being almost statistically significant $(p=.058)$. Conclusions: The prevalence for use of cervical pessary in our triplet pregnancies sample (34.4\%) is much higher than that described for twins (5\%). Even though there are significant differences for CL between groups (taking into account the potential bias for the presence of the device while making the CL measurements), the gestational age at delivery was the same for both groups. This strongly suggests that cervical pessary is an effective tool to prevent extreme prematurity in triplet pregnancies.

\section{ENVIRONMENTAL EFFECTS ON EPIGENETIC VARIABILITY: INSIGHTS FROM MONOZYGOTIC TWIN-BASED STUDIES}

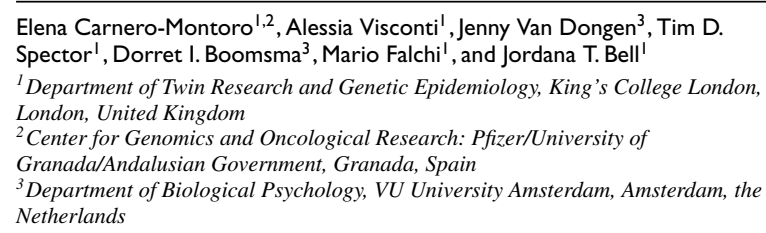

Cells have the ability to respond to environmental exposures by changing their epigenetic states and altering their transcriptional programs. Due to the modifiable and reversible nature of epigenetic variation, there is an increasing belief that genomic regions exhibiting epigenetic plasticity play a key role in the response and adaptation of individuals to changing environments and in triggering pathological conditions. The study of epigenetic variation is taking center stage in epidemiological research aiming to reveal the molecular disease effectors of genes and environment. In recent years, epigenome-wide association studies (EWAS) have confirmed in numerous large-scale studies that environmental exposures have widespread effects on DNA methylation levels. Likewise, large-scale genetic studies have revealed that disease genetic variants are commonly methylation quantitative trait locus (meQTL) that impact epigenetic variation. Most EWAS and meQTL studies published to date have predominantly focused on finding mean differences in methylation levels between exposed and unexposed subjects or genotypic groups. However, environmental perturbations could not only induce a change in methylation mean levels, but may also trigger greater epigenetic variability. Further epidemiological research is needed to explore if epigenetic variable sites are associated with environmental exposures (envVMS) and genetic variants (var-meQTLs). Twins offer a unique opportunity to study how the genome responds to modifiable environmental risk factors by comparing the methylome of exposed and unexposed geneticallymatched subjects. Here,I will present EWAS studies of monozygotic twins aiming to identify envVMS in relation to cigarette smoking. Once envVMS are identified, we aim to disentangle the contribution of gene-environment interactions in shaping the epigenetic variance differences at envVMS, and explore their possible consequences on metabolic outcomes. Our results illustrate how epigenetic varia- tion can mediate phenotypic outcomes in response to environmental exposures and how genetic variation can further modulate this response.

\section{ANALYSIS OF GENETIC OR ENVIRONMENTAL EFFECTS ON THE IRRITABLE BOWEL SYNDROME ASSOCIATED GUT MICROBIOME WITH KOREAN HEALTHY TWIN REGISTRY \\ Hang A Park ${ }^{1,2}$ and Joohon Sung ${ }^{2}$ \\ ${ }^{I}$ Department of Emergency Medicine, Hallym University Dongtan Sacred Heart Hospital, Gyounggi, Korea \\ ${ }^{2}$ Genome Epidemiology, Department of Epidemiology, Graduate School of Public Health, Seoul National University, Seoul, Korea}

Introduction: Irritable bowel syndrome (IBS) is a common gastrointestinal (GI) disorder, but its pathophysiology is not well understood. Based on recent studies, gut microbiota is thought to play important roles in the pathogenesis of IBS. However, it is still unclear whether difference in gut microbiota affecting the development of IBS is genetic or environmental. Here, we investigated the gut microbiome differences between IBS case and control groups and we tried to find out whether these differences were caused by environmental or genetic influences. Materials and Methods: The participants were recruited from the Korean Healthy Twin Study. IBS was diagnosed according to the Rome III criteria, and participants with organic GI disorder were excluded. Fecal samples were obtained from the subjects and 16S ribosomal RNA gene pyrosequencing was used to analyze the samples. Sequences were processed with VSEARCH 1.9.10, an open-source implementation of USEARCH (Caporaso et al., 2010; Edgar, 2010; Rognes et al., 2016), by using de novo OTU calling approach. For downstream analysis, OTU were collapsed and filtered using PhyloSeq (McMurdie and Holmes, 2013). We extracted 121 unrelated individuals and compared the differential abundance between IBS case and control groups. Paired $t$ test was performed to test whether there was a diffenence in abundance of the OTU with significant (FDR adjusted $p<.05$ ) difference between 18 pairs of IBS discordant monozygotic (MZ) twins. Heritability estimates of these gut microbes were calculated by variance component methods using Sequential Oligogenic Linkage Analysis Routines (SOLAR, version 7.2.5; Southwest Foundation for Biomedical Research, San Antonio, TX, USA). Results: We found that some gut microbiome was significantly different in abundance between IBS case and control groups. Two of the taxonomic branches were identified as positively significant in IBS groups: Firmicutes/ Clostridia and Bacteroidetes/ Bacteroidia/ Bacteroidaceae. In the paired $t$ test results with IBS discordant MZ twins, abundances of the microbiome were not significantly different. In addition, heritability analysis revealed genetic and shared environmental impacts on the gut microbiome. Conclusion: This study showed that abundances of human gut microbiome associated with IBS were not different between IBS discordant MZ twins and some of them are affected by host genetics.

\section{PATHOLOGICAL UMBILICAL ARTERY DOPPLER FLOW FINDINGS IN MONOCHORIONIC TWIN PREGNANCIES BETWEEN 16 AND 22 WEEKS OF GESTATION: DOES IT REALLY MATTER?}

Agata Ingala, Silvana Arduino, Giulia Benedetto, Carlotta Bossotti, Elisabetta Cantanna, Pietro Gaglioti, Enrico Libanori, Elsa Viora, and Tullia Todros Obstetrics and Gynecology Department, Sant'Anna University Hospital, Turin

Introduction: To evaluate whether absent or reverse umbilical artery (UA) Doppler flow findings, recorded between 16 and 22 weeks of gestation (and no longer in the following check) in physiological monochorionic diamniotic (MCDA) twin pregnancies, correlates with adverse outcomes such as developing of twin-to-twin transfusion syndrome (TTTS), twin anemia polycythemia sequence (TAPS) or intrauterine growth restriction of one (selective IUGR) 
or both twins. Materials and Methods: The study design was retrospective and it included 111 MCDA twins. UA end-diastolic flow was defined as Doppler waveform positive, persistently/ intermittently absent or persistently/intermittently reverse. It was recorded at first examination and monitored weekly until delivery if pathological (i.e., persistently/intermittently absent or persistently/intermittently reverse), or otherwise biweekly. IUGR was defined as an estimated fetal weight (EFW) at or below the 10th percentile for gestational age according to Hadlock curves. Exclusion criteria were: TTTS, TAPS, IUGR on of one or both twins and major malformations diagnosed before or at 16 weeks of gestation. Results: Five women were excluded because they reported pathologies enlisted in the exclusion criteria. Between 16 and 22 weeks of gestation, positive UA Doppler waveform was found in 95 cases out of 106 (Group A); the remaining 11 demonstrated persistently/intermittently absent or persistently/intermittently reverse UA Doppler waveform (Group B). In Group A we found 20 sIUGR Type1 (21\%), 8 IUGR of both twins (8\%), 10 TTTS (10\%) and 1 TAPS (1\%).In Group B UA Doppler waveform normalized after 22 weeks of gestation and only 1 woman (2\%) developed sIUGR Type1. Conclusions: Early findings of persistently/ intermittently absent or persistently/intermittently reverse UA Doppler waveform, if not confirmed after 22 weeks of gestation, do not seem to correlate with adverse outomes. However, further research is required to confirm and expand these results in a larger cohort.

\section{ASSOCIATION BETWEEN PAPP-A LEVELS DURING THE FIRST TRIMESTER AND ADVERSE OBSTETRICAL OUTCOMES IN TWIN PREGNANCY}

Giulia Benedetto, Silvana Arduino, Carlotta Bossotti, Elisabetta Cantanna, Agata Ingala, Enrico Libanori, Elisabetta Muccinelli, Elsa Viora, and Tullia Todros

Obstetrics and Gynecology Department, Sant'Anna University Hospital, Turin, Italy

Introduction: In singleton pregnancies low PAPP-A levels are known to correlate with adverse outcomes.Despite being more at risk of obstetrical complications,twin pregnancies have been excluded from the vast majority of studies and the few ones in literature have reached different conclusions. The main problems are the difficulty to establish cut-off values for PAPP-A and the uncertain impact of chorionicity on maternal serum levels. The aim of this work was first to investigate whether chorionicity significantly affects PAPP-A values and second, the relationship between serum PAPP-A levels and adverse outcomes in twin pregnancy, both from the maternal and from the fetal-neonatal side. Materials and Methods: For our retrospective study we selected 717 patients (549 dichorionic and 168 monochorionic) among 1,305 women with multiple gestations that came to Sant' Anna Hospital (Turin) between May 25, 2011 and August 29, 2014 to have a combined or integrated test measuring PAPP-A levels between $11+0$ and $13+6$ weeks of gestational age. Exclusion criteria were: higher-order multifetal pregnancies and childbirth carried out elsewhere. We considered as low levels of PAPP-A values those below the $5^{\circ}$ and the $10^{\circ}$ centile of our series of data. Results: Median concentration of PAPP-A serum levels in dichorionic pregnancies was $1.44 \mathrm{MoM}$, whereas in monochorionic $1.06 \mathrm{MoM}(p<.0001)$ :the latter thus present statistically lower levels. Considering only the subgroup of dichorionic pregnancies, as far as the maternal complications were concerned, we could not observe an association between low PAPP-A values and hypertensive disorders, whereas there was an increase of risk of very preterm ( $<32$ weeks), preterm ( $<37$ weeks) birth and total adverse events, though not reaching statistical significance. As far as fetalneonatal complications were concerned, we could observe a tendency, without reaching statistical significance, towards disorders in fetal growth and total adverse events in the group of pregnancies with low PAPP-A values. Conclusions: Chorionicity significantly influences PAPP-A serum concentrations. Contrary to singleton pregnancies, currently available data do not seem to indicate the need of ulterior diagnostic investigations or a more thorough monitoring following the observation of low PAPP-A levels in the first trimester of pregnancy, considering that the current management of twin pregnancy already aims at early identification and prevention of these adverse events.

\section{CERCLAGE VERSUS PESSARY TO PREVENT PRETERM BIRTH IN TWIN PREGNANCIES AND SONOGRAPHIC SHORT CERVIX}

Marta Ruiz García, Jesús Zabaleta Jurío, Carlos Larrañaga Azcárate, Irati Zabaleta Loinaz, Begoña Gastón Moreno, Natalia Franco Abián, Julen Barrenetxea Arrinda, Andrea Safont, Marta Bazán, and Itxaso Pedroarena

Obstetrics and Ginecology, Complejo Hospitalario de Navarrra, Pamplona, Navarra, Spain

Introduction: Spontaneous preterm birth is the leading cause of perinatal morbidity and mortality, the rate of this complication being much higher in twins than in singletons; therefore, interventions to prevent preterm birth in this population are needed. Objective: The objective was to compare the effectiveness between cerclage and cervical pessary in preventing preterm birth in twin gestations with transvaginal ultrasound short cervix $(\leq 25 \mathrm{~mm})$. Primary outcome was preterm birth $<34$ weeks. Material and Methods: This was a retrospective trial of twin pregnancies from $15+0$ to $27+6$ weeks' gestation with transvaginal short cervix $(\leq 25 \mathrm{~mm})$ from 2002 through June 2017. The findings were detected both by regular ultrasound exploration and due to symptoms referred by patients. Cerclage or cervical pessary placement were offered, except when amniotic membranes were visible, a situation in which the pessary is contraindicated. Results: 65 subjects were studied, 50 underwent cerclage (of which 21 presented visible membranes) and 15 received a cervical pessary. One of the latter was lost to follow-up. There were no significant differences between the cerclage and the pessary in rates of gestational age at treatment ( $23+0$ vs. $21+5$ weeks); previous parity ( 82 of former were nulliparous vs. $86.7 \%$ ); cervix length at treatment (15.6 vs. $16.96 \mathrm{~mm})$; medium gestational age at birth $(32+5$ vs. $30+6$ weeks; or rate of cesarean delivery ( 48.0 vs. $35.7 \%)$. There were no significant differences between both devices in rates of preterm birth $<34$ weeks ( 46.0 vs. $50 \%$ ). Studying by gestational age periods, we found there were significant differences at very early weeks of pregnancy. If we assessed all subjects regardless of exposure of membranes, we obtained 2 vs. $21.4 \%$ of miscarriages in pregnancies of less than 20 weeks; there are no differences in the rest of periods. However, if we made two groups excluding those cases with membranes visible, a difference existed at ages less than 22 weeks. Conclusions: In women with twin pregnancy at our service, we obtain better results with significant difference using cerclage at very immature gestational ages. Nevertheless, there were no significant differences at higher gestational ages because of the small number of cases.

\section{DOUBLE CERCLAGE IN TWIN PREGNANCY}

Marta Ruiz García, Irati Zabaleta Loinaz, Natalia Abián Franco, Begoña Gastón Moreno, Marta Bazán; Itxaso Pedroarena Lecumberri, Andrea Safont, and Julen Barrenetxea Arrinda

Obstetrics and Ginecology, Complejo Hospitalario de Navarra, Pamplona, Navarra, Spain

Introduction: The risk of preterm birth is increased in multiple pregnancies. Cervical cerclage is a surgical procedure carried out during the pregnancy to try to prevent preterm birth by limiting shortening and opening of the cervix. We are presenting a case of twin pregnancy in which was performed cerclage twice. Case Report: A 35year-old woman at her third pregnancy. Quadruplet gestation was obtained by assisted reproduction techniques and reduction of two of the embryos was performed, resulting in bichorionic biamniotic twin pregnangy. As obstetric antecedents, first pregnancy at term but 
second one with death baby due to very preterm birth. At week 12 and due to the suspicion of cervical incompetence, there was done prophylactic cerclage. At week 22 the patient started feeling unspecific abdominal discomfort. During the obstetric assessment it was found short cervix by transvaginal ultrasound $(19 \mathrm{~mm})$ with slightly dilated canal and funneling of $5 \mathrm{~mm}$. Apart from that, there were registered uterine contractions at monitoring. The woman was admitted at the hospital with tocolytic drugs for inhibition of acute preterm labor (rectal nifedipine) in order to perform an emergency cerclage the day after if uterine contractions have stopped. Emergency cerclage was done successfully. At week $27+6$, she asked again about uterine contractions. This time, cervical length was normal $(31 \mathrm{~mm})$; nevertheless, she was admitted at hospital to observe her evolution and under treatment of progesterone. At 30+4 weeks membranes ruptured, so corticosteroid therapy was administered to reduce the incidence and severity of respiratory distress syndrome and mortality in case of preterm birth. In addition, tocolytic drugs were needed to stop uterine contractions (atosiban and nifedipine). At week $31+1$ the patient called when she noticed the feet of one baby in the vagina; an urgent cesarean section was performed because of breech presentation of first baby and its prematurity. A male was born first weighing 1,570 grams with Apgar 9 at the first minute and 9 at 5 minutes, pH 7.14 and 7.26 (arterial and venous respectively). Afterwards, a male weighing 1,425 grams with Agpar of 7 and 8 (at first and 5 minutes respectively), and $\mathrm{pH} 7.31$ and 7.41 (arterial and venous respectively). Conclusions: The effectiveness and safety of cervical cerclage has been demonstrated for single gestations but it remains uncertain for multiple ones. It is necessary to make more trials about cerclage and twin gestations.

\section{MONOCHORIONIC DIZYGOTIC TWINS ARE NOT RARE: EVIDENCE FROM A TWIN BIRTH COHORT}

\author{
Jeffrey M Craig' , Richard Saffery' ', Yuk Jing Loke' ${ }^{\text {, and Mark Umstad }}{ }^{2}$ \\ ${ }^{1}$ Murdoch Childrens Research Institute, Royal Children's Hospital, Parkville, \\ Melbourne, Victoria, Australia \\ ${ }^{2}$ Department of Obstetrics and Gynaecology, University of Melbourne, Royal Women's \\ Hospital, Melbourne, Victoria, Australia
}

Introduction: Monochorionic (MC) placentation has indicated monozygosity for generations of obstetricians. This rule has been considered so robust that almost all parents of twins with a single placenta diagnosed on first-trimester ultrasound have been advised that no further testing is ever required to prove their twins' monozygosity. In recent years, there have been a number of reports of monochorionic dizygotic (MCDZ) twins, almost exclusively following treatment with assisted reproductive technologies, often involving microinjection techniques. In a longitudinal birth cohort of 250 mothers and their twins, we performed zygosity testing of 69 twin pairs recorded as MC from first trimester ultrasounds and/or placental examination at birth. Materials and Methods: Zygosity testing was determined by a 12 marker microsatellite test at the Australian Genome Research Facility, Melbourne. For twins where tests indicated a DZ status, testing was performed again on independent sample where possible. Results: We found that 2/69 (2.9\%) MC twin pairs were DZ, only one of which was conceived through IVF. We also found 6/69 (8.7\%) MCMZ pairs for which first trimester ultrasounds indicated DC but were MC on placental examination at birth. Conclusions: The frequency of DZ twinning in $\mathrm{MC}$ pregnancies may be as high as $3 \%$. We recommend that parents are informed that this uncommon but not rare event. We also recommend routine placental examination and reporting of chorionicity at birth, and that zygosity testing is recommended to parents of MC twins who subsequently report major phenotypic discordance.

\section{A STUDY OF PENETRANCE FOR BREAST CANCER IN IDENTICAL TWIN PAIRS}

Eunjung Lee, Amie Hwang, Wendy Cozen, Ann Hamilton, and Thomas Mack Department of Preventive Medicine, Keck School of Medicine, University of Southern California, USA

Background: In 2003 (Hamilton et al., NEJM) we studied female twins with breast cancer. Among discordant pairs, familiar behavioral and reproductive factors such as age at menarche and age at first term pregnancy significantly predicted diagnosis $(O R$ 1.2$1.5)$ as expected within $670 \mathrm{DZ}$ pairs but not $(O R$ 1.0-1.1) within $843 \mathrm{MZ}$ pairs. Among $209 \mathrm{MZ}$ pairs concordant for breast cancer but discordant for age at diagnosis (by years to decades), earlier age at diagnosis was significantly predicted independently by several markers of earlier puberty $(O R=3-5)$, especially if puberty was very early. This finding of substantial biological and practical significance could not be explained on non-biological grounds, but has not been reproduced or acted upon in the laboratory. Methods: We propose to do reproduce the earlier study, and to assess the reproductive and behavioral determinants of breast cancer in the context of genotype. We have designed and tested a web-based questionnaire which takes 30 minutes (non-cases) to 60 minutes (cases) to complete. By screening a large roster of volunteer breast cancer cases and participants in the California Twin Program we have identified 700 additional affected twin pairs and expect to identify at least an additional 300 such pairs. Among the 110 affected twins first contacted, 80 completed the questionnaire within 10 days. MZ pairs concordant for breast cancer are the least prevalent and most informative subset, and we may have difficulty accruing our goal of 200 such pairs. We welcome collaborations and referrals of such pairs, if kept unaware of hypotheses)

\section{DETERMINANTS OF CONCORDANCE FOR AGE AT MENARCHE AMONG TWINS}

Deboshree Roy, Amie Hwang, Wendy Cozen, Ann Hamilton, and Thomas Mack Department of Preventive Medicine, Keck School of Medicine, University of Southern California, Los Angeles, California, USA

Background: Several published studies link earlier puberty to increased risks of breast cancer, psychiatric disorders, personalityrelated disorders such as bulimia and substance abuse. While early hormonal maturity clearly has substantial genetic determination, trends in time and space suggest that unknown environmental factors also determine age at menarche. Methods: The California Twin Registry includes 3,369 pairs of MZ and 3,030 pairs of DZ female twins. Self-responses of each twin's age at menarche were used to determine the difference in age at menarche year. Pair-specific maternal and familial individual responses from the same registration questionnaire were also dichotomized. When twins disagreed, we constructed two alternative subsets, one ignoring the lowest and one ignoring the highest individual estimate. We calculated the odds ratios for the effect of each factor on the discordance for age at menarche within each of the three data sets. A linear regression was used to assess the interval between paired ages at menarche. Results: In the subset of pairs who were discordant for age at menarche (i.e., with an interval $>1$ year) and who agreed on the given family characteristic, age-discordant pairs were found to have mothers with more education (OR 1.28; 95\% CI [1.03, 1.59]) and, independently, more biological brothers $(O R 1.35 ; 95 \%$ CI $[1.04,1.76])$ than those concordant for age at menarche. The same results, with nearly identical odds ratios, were found after the analysis of results from each data set based on paired twins who disagreed on the family characteristic. A longer interval between paired ages was associated with higher birth order. No factor was found associated with concordance for age at menarche among MZ twins and neither the number of biological sisters, the maternal age at birth, nor parity was associated 
with discordance for age at menarche among DZ pairs. Conclusions: Maternal education, birth order, and the number of biological brothers are reliably reported by respondents, and each is clearly behaviorally and biologically meaningful. Birth order and maternal age are known to be associated with DZ twinning. No other explanatory hypothesis presents itself. Based on the MZ twins, no reliably non-genetic determinants of age at menarche were found.

\section{GENOME AND EPIGENOME FINDINGS FOR WELLBEING}

B. M. L. Baselmans, R. Jansen, H. F. Ip, J. van Dongen, A. Abdellaoui, M. P. van de Weijer, G. Willemsen, J.J. Hottenga, E. J. C. de Geus, D. I. Boomsma, M. G. Nivard, and M. Bartels

Vrije Universiteit Amsterdam, the Netherlands

Introduction: Most existing research on the genetics of wellbeing is characterized by a focus on individual phenotypes, despite the strong correlations between related traits. The high genetic correlations $(|\mathrm{rg}|>.75)$ between life satisfaction, positive affect, neuroticism, and depressive symptoms suggests common underlying biology. Acknowledging this overlap, we performed a multivariate genome-wide meta-analysis of these four phenotypes (collectively referred to as the wellbeing spectrum) to increase the power to identify associated genetic variants. Method: Our analyses leveraged univariate GWAMA of life satisfaction (Nobs $=80,852$ ), positive affect $($ Nobs $=189,028)$, neuroticism $($ Nobs $=238,315)$, and depressive symptoms (Nobs $=449,954)$ (Brice et al., 1993; Hek et al., 2013; Hyde et al., 2016; Lo et al., 2016; Okbay et al., 2016). The dependence between effect sizes induced by sample overlap was estimated using LD score regression. Knowledge of the error correlation between univariate meta-analyses allowed dependent samples to be meta-analyzed. In a similar fashion, we proceeded to aggregate the effect across multiple SNPs based on prior knowledge that some of these SNPs collectively influence the expression level of a gene transcript or the methylation level at a $\mathrm{CpG}$ site. In this procedure, known as summary based transcriptome-wide and methylation-wide association analyses (TWAS and MWAS), we imputed the effect of changes in gene expression or $\mathrm{CpG}$ site methylation on the wellbeing spectrum. Information on the relation between SNP and $\mathrm{CpG}$ methylation or gene expression were obtained from the summarized results of cis-expression (e)QTL and cis-methylation (m)QTL studies in whole blood. Results: Multivariate genome-wide analyses $($ Nobs $=958,149)$ of the wellbeing spectrum, revealed 63 significant independent signals. TWAS and MWAS analyses uncovered 34 transcript-trait associations and $115 \mathrm{CpG}$ methylation-trait that reached genome-wide significance at a Bonferroni corrected level. Annotation of individuals genes (Welter et al., 2014) identified in G/T/M-WAS revealed abundant evidence for possible pleiotropic effects, but also genes that have not previously been associated with any trait. Discussion: In summary, while previous univariate analyses of phenotypes in the wellbeing spectrum were moderately successful, we gained power by use of multivariate analysis. Our work shows definitive progress in the genetics of wellbeing.

\section{THE GENETICS OF DIZYGOTIC TWINNING: THE TWINNING GENETICS CONSORTIUM}

\section{Hamdi Mbarek on behalf of the Twinning Genetics Consortium}

Netherlands Twin Register, Queensland Twin Registry, Finnish Twin Cohort, Minnesota Center for Twin and Family Research, deCODE, Twins UK, Twins Early Development Study, Swedish Twin Registry, Women's Genome Health Study, Avera Twin Register, Danish Twin Register

Introduction: The tendency to conceive spontaneous dizygotic (DZ) twins is a complex trait that aggregates in families, and there is evidence that it is at least in part genetically determined. Although the fundamental physiological mechanism for DZ twinning is the release and fertilization of multiple oocytes, for which both animal models and human data suggest multifactorial inheritance, the underlying molecular basis of the trait is incompletely understood. We set up the Twinning Genetics Consortium (http://www. twinningconsortium.org/) to characterize the genetic basis of twinning in humans and published our first findings in 2016. Materials and Methods: We have collected over 35,000 individuals of European ancestry, representing the largest twins cohorts in the world. With this resource, we have performed the first genome-wide association study (GWAS) for being a mother of DZ twins in 1,980 mothers of spontaneous DZ twins and 12,953 controls of European ancestry from the Netherlands, US, and Australia. A total of 9,073,348 SNPs after imputation against the $1000 \mathrm{G}$ reference set were tested. Findings were replicated in a large, independent Icelandic cohort consisting of 3,597 mothers of twins and 297,348 controls and tested for association across a broad range of fertility traits in women. The consortium is now recruiting and genotyping more mothers of DZ twins and extending the analyses to include 'being a twin' as a phenotype as well. Results: We have obtained the first genome-wide significant findings for DZ twinning. Two SNPs were identified near FSHB (rs11031006, $p=1.54 \times 10-9)$ and SMAD3 (rs17293443, $p=1.57 \times 10-8)$ and replicated in an independent cohort ( $p=3 \times 10-3$ and $\mathrm{p}=1.44 \times 10-4$, respectively). Based on $\sim 90,000$ births in Iceland, the relative risk of a mother delivering twins increased by $18 \%$ for each copy of the rs $11031006-\mathrm{G}$ allele and $9 \%$ for the rs $17293443-\mathrm{C}$ allele. A higher polygenic risk score (PRS), or cumulative genetic risk profiles from across the genome, for DZ twinning, was calculated based on the results of the DZ twinning GWAS and was significantly associated with DZ twinning in Iceland $(p=.001)$. A higher PRS was also associated with having children $(p=.01)$, greater lifetime parity $(p=.03)$, and earlier age at first child $(p=.02)$. The rs $11031006-\mathrm{G}$ allele was associated with higher serum follicle-stimulating hormone (FSH) levels, earlier age at menarche, earlier age at first child, higher lifetime parity, lower polycystic ovary syndrome risk, and earlier age at menopause. Conversely, the rs17293443-C allele was associated with later age at last child. Preliminary results for the 'being a twin' phenotype indicates replication of these findings. Conclusions: This study extends our understanding of the control of natural multiple follicle growth and reproductive aging. This new knowledge will contribute to the development of novel ways to manipulate ovarian functioning, which is crucial for the treatment of infertility as well as for contraception.

\section{ON FAMILIAL RISK OF MULTIPLE CANCERS: DOES BRAIN TUMOR OCCURRENCE RELATE GENETICALLY TO OTHER CANCERS?}
J. Hjelmborg ${ }^{4,5}$,J. Kaprio ${ }^{1,2}$, T. Korhonen ${ }^{1,2,3}$, La Mucci ${ }^{7,8}$, K. Christensen ${ }^{4,5}$, H.O. Adami ${ }^{10, i_{1},}$, T. Scheike ${ }^{6}$, and J. R. Harris ${ }^{9}$
${ }^{I}$ Institute for Molecular Medicine, University of Helsinki, Helsinki, Finland ${ }^{2}$ Department of Public Health, University of Helsinki, Helsinki, Finland ${ }^{3}$ Institute of Public Health and Clinical Nutrition, University of Eastern Finland, Kuopio, Finland
${ }^{4}$ Department of Epidemiology, Biostatistics and Biodemography, University of Southern Denmark, Odense, Denmark
${ }^{5}$ The Danish Twin Registry, University of Southern Denmark, Odense, Denmark ${ }^{6}$ Department of Biostatistics, University of Copenhagen, Copenhagen, Denmark ${ }^{7}$ Channing Division of Network Medicine, Brigham and Women's Hospital, Harvard Medical School, Boston MA, USA
${ }^{8}$ Centre for Public Health Sciences, University of Iceland, Reykjavik, Iceland ${ }^{9}$ Department of Genetic Research and Bioinformatics, The Norwegian Institute of Public Health, Oslo, Norway
${ }^{10}$ Department of Medical Epidemiology and Biostatistics, Karolinska Institutet, Stockholm, Sweden
${ }^{1 I}$ Department of Epidemiology, Harvard School of Public Health, Boston, MA, USA

Twin studies provide insight into familial risk of cancer by leveraging the unique genetic relatedness of twins. We considered familial brain and CNS cancer occurrence in the Nordic population based cohort of 202,868 monozygotic and dizygotic twins with more than 40 years of follow-up via nationwide cancer registries. We found only four pairs (1 MZ and $3 \mathrm{DZ}$ pairs) concordant for brain and CNS cancers, corresponding to what would be expected by chance. How- 
ever, extending analyses to cross-cancer (brain with other cancers) occurrences reveals a picture of risk partly influenced by genetic factors. For instance, the risk is doubled if prostate cancer occured during life in the co-twin. We address cross-cancer risks for brain cancer with multiple cancers to disentangle genetic relatedness across age.

\section{CPGFORID: EPIGENETIC DISCRIMINATION OF IDENTICAL TWINS UNDER THE FORENSIC SCENARIO}

Athina Vidaki', Elena Carnero-Montoro ${ }^{2}$, Ruben Boers ${ }^{3}$, Joachim Boers ${ }^{3}$ Celia Diez Lopez', Vivian Kalamara', Timothy Spector ${ }^{2}$, Joost Gribnau ${ }^{3}$, Jordana Bell ${ }^{2}$, and Manfred Kayser'

${ }^{I}$ Department of Genetic Identification, Erasmus MC University Medical Center Rotterdam, Rotterdam, the Netherlands

${ }^{2}$ Department of Twin Research and Genetic Epidemiology, King's College London London, United Kingdom

${ }^{3}$ Department of Developmental Biology, Erasmus MC University Medical Center Rotterdam, Rotterdam, the Netherlands

Introduction: Standard DNA profiling is typically non-informative for differentiating between monozygotic (MZ) twins. Whole genome sequencing was recently proposed as a solution; however, both the rarity of such autosomal SNPs and required ultra-high coverage resulting in immense costs, make this approach impractical. Epigenetic variation was identified as suitable source for MZ twin differentiating biomarkers; nevertheless, studies addressing the forensic feasibility are lacking. Materials and Methods: Here, we performed the first use of DNA methylation for MZ twin differentiation under the forensic scenario, comprising the i) discovery of twin-differentially methylated sites (tDMSs) in reference-type DNA via genome-wide analysis (HumanMethylation450 BeadChips, Illumina), ii) validation of candidate tDMSs using forensically suitable, targeted methods (methylation-specific quantitative PCR), and iii) analysis of validated tDMSs in trace-type DNA using the same targeted methods. Whole blood and buccal cells of $10 \mathrm{MZ}$ twin pairs (female, aged 52-62 years old) were analyzed together with forensic-type material including minute bloodstains and cigarette butts. Results: Genome-wide methylation analysis covering $2 \%$ of all CpGs, identified hundreds of twin pair-specific tDMSs showing 0.15 methylation differences in both tissue types. In blood DNA we identified on average 56 tDMSs per pair with $>0.3$ twin-totwin methylation differences. Across all pairs, $67.9 \%$ of the top three, twin-specific blood candidate tDMSs generated $>0.1$ methylation differences in reference blood samples, and from the validated tDMSs $68.4 \%$ resulted in $>0.1$ differences in bloodstains using methylation-specific qPCR. Applying an updated marker selection strategy, we analysed 8 additional tDMSs for an example MZ pair; $87.5 \%$ of which were positively validated in both referenceand trace-type blood DNA. Following the revised selection approach we expanded our targeted investigation in buccal cells of one example twin pair only. In this case, $27.2 \%$ and $50 \%$ of the selected 25 candidate tDMSs in buccal cells generated $>0.1$ methylation differences in reference saliva samples and cigarette butts, respectively. Aiming to overcome restrictive marker selection via epigenome screening of both reference and trace-type samples, we applied a novel, highly sensitive enzymatic-based methylation DNA sequencing approach (MeD-seq) covering $\sim 50 \%$ of all CpGs with promising preliminary results. Conclusions: The observed discrepancies between microarray and qPCR results can be explained by cell composition differences, method-to-method variation and other technical reasons like bisulfite conversion inefficiency depending on input DNA amounts. Demonstrating that a proportion of tDMSs selected from reference-type samples eventually becomes non-informative in trace-type samples as well as the influence of the DNA source questions the number of markers needed for successful MZ twin differentiation and identification.

\section{MAPPING RISK FROM GENES TO BEHAVIOR}

\section{Danielle M. Dick}

Virginia Commonwealth University, Richmond, Virginia, USA

Irv Gottesman is widely remembered for bringing the endophenotype construct to psychiatry, which opened an exciting new area of study, and led to deep discussions about the utility of the endophenotype construct in gene identification. In this talk I will discuss how interest in endophenotypes more broadly has raised awareness about the importance of understanding the mechanisms by which genes affected psychiatric outcome and how that continues to live on and grow.

\section{PROVIDING FEEDBACK VIA A PARTICIPANT PORTAL: EXPERIENCES FROM THE NETHERLANDS TWIN REGISTER}

Gonneke Willemsen, Quinta Helmer, Reena Luijten, Roos van der Deijl, Sara Khosdelazad, Margot van der Weijer, Bart Baselmans, Dorret Boomsma, and Meike Bartels

Netherlands Twin Register, Department of Biological Psychology, Vrije Universiteit Amsterdam, the Netherlands

Five years ago the Netherlands Twin Register (NTR), with funding from BBMRI-NL (www.bbmri.nl), started to provide its participants with personalized feedback via the MyNTR portal (www. tweelingenregister.org/mijnntr). Beginning with small groups, we continued to invite participants so that at present the majority of adult twins and their family members, provided they are registered with a personal email address, received their login details. After activating their account, participants have access to their own personal reports, in which their information is compared against a general background of other NTR participants. As a next step, the NTR recently began extending portal access to the parents of young participants, who also receive feedback based on the data they themselves provided. Here we share our experiences with the MyNTR portal and present the results of a small survey among participants in which they provided their opinion about the portal and among those who did not yet activated their portal account. Information on portal usage among participants is updated daily and can be linked to participants characteristics such as age and sex but also to information known via the surveys which were completed as part of the NTR's longitudinal survey projects. Upon visiting the portal, participants were asked to answer a few questions regarding the portal setup and intended use. Participants who did not activate their portal account but who did complete a survey sent by email were asked for their reason not to activate the portal. The first results of the survey among portal participants indicates that they are generally happy with the content of the portal. Participants read personal feedback and survey information, but less often research information and there is no information participants do not want to know. If possible, they want all the information available. Almost all participants want to receive regular updates via email. All participant responses demonstrate that personalised feedback is very much welcomed by participants. However, cohorts are advised to consider IT and personnel requirements prior to starting a participant portal.

\section{MULTI-VARIATE, MULTI-RATER, MULTI-AGE GWAS OF LONGITUDINAL AGGRESSION AND ATTENTION PROBLEMS}

Dorret I. Boomsma

Vrije Universiteit, Amsterdam, Netherlands, on behalf of the ACTION consortium

Twin cohorts provide unique opportunities for investigations of the role of genetics in the etiology of complex human traits. Twin cohorts tend to be population based, often have rich longitudinal follow-up and increasingly collect DNA, RNA and biomarker 
data. The EU-funded ACTION consortium (Aggression in Children: Unraveling gene-environment interplay to inform Treatment and InterventiON strategies) includes eight twin cohorts (from Scandinavia, the Netherlands, Australia and the United Kingdom) which have phenotyped over 90,000 young twins for longitudinal aggressive behavior and attention problems. The twin data revealed that genes substantially contribute to variation in aggression (AGG). The most important co-occurring behavioral problems with aggression in children and adults are attention (ATT) problems. Analyses of longitudinal observations of AGG and ATT revealed substantial stability with a significant portion of this stability attributable to genetic factors. In children, behavior is often assessed by parental or teacher ratings, leading to multivariate data, whose covariation is mainly due to genetic correlations. Genome-wide association studies (GWAS) in children have considered AGG and ATT in univariate analyses, as have most other GWA meta-analyses projects either in children or in adults. Twin studies have been at the forefront in leveraging the analyses of multivariate and longitudinal data, but GWA stud- ies have so far made use of these approaches in a limited way. The analysis of multiple measures in GWA studies is non-trivial as repeatedly including the same subject in meta-analysis inflates type- 1 error. The ACTION consortium is, in collaboration with other child cohorts that have phenotype and genotype data, carrying out a multivariate GWAS of the (developmental) genetic etiology of AGG and ATT in 30,000 children. A multivariate meta-regression model is used (Nivard et al., Genetic Overlap Between Schizophrenia and Developmental Psychopathology: Longitudinal and Multivariate Polygenic Risk Prediction of Common Psychiatric Traits During Development. Schizophr Bull. 2017; doi: 10.1093/schbul/sbx031), which relies on a series of univariate GWASs of longitudinal phenotypes within cohorts. The multivariate analysis is performed at the metaanalysis stage, omitting the need for multivariate modeling in individual cohorts. The approach enables the inclusion of dependent measures into a single analysis without inflating the false positive rate and allows for the estimation of age, rater, instrument or cohort specific effects for individual SNPs. 